
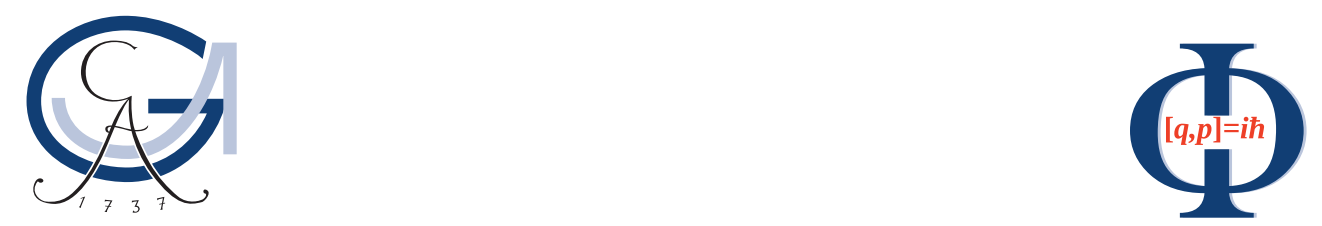

\title{
Kinetics of Directed Self-Assembly of Block Copolymers via Continuum Models
}

\author{
Dissertation \\ zur Erlangung des mathematisch-naturwissenschaftlichen \\ Doktorgrades \\ "Doctor rerum naturalium" \\ der Georg-August-Universität Göttingen \\ im Promotionsprogramm ProPhys \\ der Georg-August University School of Science (GAUSS) \\ vorgelegt von \\ Juan Carlos Orozco Rey \\ aus Bogotá, Colombia
}

Göttingen, 2018 


\section{Betreuungsausschuss}

Marcus Müller Institut für Theoretische Physik Georg-August-Universität

Annette Zippelius Institut für Theoretische Physik Georg-August-Universität

\section{Mitglieder der Prüfungskommission}

Referent:

Marcus Müller

Institut für Theoretische Physik

Georg-August-Universität

Korreferentin: Annette Zippelius

Institut für Theoretische Physik

Georg-August-Universität

Weitere Mitglieder der Prüfungskommission:

Kostas Ch. Daoulas

Theory Department

Max Planck Institute for Polymer Research

Matthias Krüger

Institut für Theoretische Physik

Georg-August-Universität

Herre Jelger Risselada

Institut für Theoretische Physik

Georg-August-Universität

Cynthia A. Volkert

Institut für Materialphysik

Georg-August-Universität

Tag der mündlichen Prüfung: 06.02.2019 


\section{Agradecimientos}

Esta investigación es el resultado de mi inherente curiosidad y mi obstinada personalidad, y no hubiera sido posible sin la presencia de diferentes personas que en los últimos 5 años me han inspirado y apoyado de diversas maneras.

La aventura académica con la cual inicié el proyecto doctoral que dio luz a esta investigación, comenzó con una conversación casual con el director del programa de maestría biomédica del cual yo hacia parte en 2013. Un café con él se convirtió en una profunda conversación sobre la vida y cómo tomar decisiones. De esa conversación me quedaron tres simples consejos: hay personas que siempre alaban lo que usted hace, le dan palmadas en la espalda y le dicen que todo lo que usted dice está bien, manténgase alejado de esas personas porque ellas no le ayudan a crecer. El segundo fue procure rodearse de personas que tienen puntos de vista diferentes e incluso opuestos a los suyos y busque discutir los problemas que va a encontrar en su carrera científica con ellos en lugar de intentar resolverlos usted solo. El último consejo, aparentemente obvio, fue cuando deba tomar decisiones importantes busque el consejo de personas con experiencia en los temas que involucran esa decisión y no se apresure en tomar ese tipo de decisiones. Ojalá sean personas mayores que hayan tenido que tomar decisiones similares.

Sin pensarlo, Dr. Edgar Eduardo Romero Castro fué crucial en mi toma de decisiones profesionales y de vida a partir de ese momento, incluyendo venir a Europa a presentar los resultados de mi investigación durante mis estudios de maestría. Tras enviar el resumen de mi trabajo a un par de eventos científicos, fuí aceptado para presentar un poster en un taller en la ciudad de Maguncia, Alemania. Un profesor muy diligente respondió cada uno de mis muchos correos electrónicos confirmando la información y detalles sobre el evento. Desde el primer día en Maguncia, le pedí en repetidas ocasiones que viniera a ver mi poster. La sesión de posters estaba planeada para los dos últimos días del taller que duraba una semana. Dicho profesor es ahora el supervisor de mi tesis doctoral, Prof. Dr. Marcus Müller.

Con un profundo respeto, le agradezco al Prof. Müller por su tiempo, su gran paciencia, su apoyo y por la manera en la que retó mis habilidades y lim- 
itaciones durante mis estudios doctorales en Gotinga. A través de nuestra interacción, entendí y asimilé profundamente las recomendaciones del Dr. Romero y muchos rasgos de mi ser han cambiado tanto profesional como personalmente. El mundo de la física teórica que me resultaba críptico y distante tiene ahora una perspectiva diferente para mi y su belleza ha ampliado mi entendimiento y mis expectativas de la ciencia.

Mi familia ha sido la piedra angular de este viaje personal y les agradezco profundamente por entenderme y apoyarme de manera incondicional con todas sus fuerzas y sus recursos: a mi esposo, Oscar, a mi madre María, a mi hermana Mauren y a mi tío Luis. A pesar de todos los retos que hemos tenido que superar y los cambios que estos han suscitado en nuestras vidas, pronto disfrutaremos de los frutos de estos años de paciencia y esfuerzos conjuntos.

Todos los miembros del grupo de investigación del Prof. Müller han hecho de mi vida en Gotinga y de mi proyecto doctoral una experiencia realmente gratificante llena de valiosas lecciones personales y profesionales. Agradezco especialmente a Ulrich Welling y a Katrin Glormann por su tiempo y su crucial apoyo en mi llegada a Gotinga. A Weihua Li, Guojie Zhang, Ludwig Schneider y Yuliya Smirnova por las muchas discusiones científicas, espontáneas y casi interminables la gran mayoría de ellas. A Marcel Langenberg, Israel Barragan, Anton Iakolev, Veronica Chiappa y Laura Endter, ha sido un placer para mi intercambiar opiniones, experiencias y consejos en nuestras múltiples conversaciones con diversos grados de alcohol en la sangre.

A mis amigos y a mis seres queridos que me impulsaron a mantenerme enfocado y con una mente clara durante las diferentes etapas de este proyecto y con los cuales viví muchos momentos altamente gratificantes: Angélica Sandoval, Alvaro Andres Argoti, Arnd-Christian Wassmus, César Beleño, Gloria Barcenas, Julio Rios, Marleen Triebeger, Melanie Haas, Shayan Rahimian y Vanessa Pacheco. Agradezco especialmente a Gustavo Andrés Salguero López y a Julio Ríos por la bienvenida a Alemania y por hacer de ese aterrizaje en tierras extranjeras una experiencia más Colombiana.

Esta tesis doctoral fue posible gracias a la financiación de la Unión Europea bajo el acuerdo No. 619793 CoLiSA.MMP (Computational Lithography for Directed Self-Assembly: Materials, Models, and Processes), y el apoyo financiero de la escuela de ciencias de Georg-August University, GAUSS. 


\section{Acknowledgments}

This dissertation is the outcome of my inherent curiosity and my stubborn personality; it could have not been possible without the many people that throughout the last 5 years have been my support and inspiration in different ways.

The academic journey giving rise to this work started with a simple conversation with the head of the master program that I was part of back in 2013. A regular coffee-talk after a lecture turned into a striking personal learning lesson after he gave me the following three suggestions: Firstly, stay away in life from those who always praise your actions and highlight only the positive things about you, "They will not promote your growth," he said. Secondly, look for people whose points of view are different from yours and promote the discussion to address scientific challenges instead of tackling them on your own. And finally, apparently obvious, consult with experienced people, hopefully older, when you find yourself in need to take major decisions in life, do not hurry when it comes to big changes.

Without intending to do so, Dr. Edgar Eduardo Romero Castro became instrumental in how I chose the next step in my career when I decided to come to Europe to present the research results obtained in pursuing my master's degree. After sending a couple of abstracts to different places in Europe, I was accepted to present a poster in a workshop in Mainz, Germany. A very diligent professor was replying every single email that I sent requesting information to prepare my participation in that workshop. Once in Mainz, I repeatedly asked him to visit my poster from the very first day of the one-week event. The poster session took place in the last two days and he came to visit mine. He is now the main supervisor of my doctoral thesis, Prof. Dr. Marcus Müller.

With deep respect, I am grateful to Prof. Müller for his time and patience, his support, and how he has challenged me in conducting my doctoral studies in Göttingen. Through you and our interaction, I have come to deeply understand 
the recommendations given by Dr. Romero and I have changed many aspects of my professional and personal self. The world of theoretical physics, once cryptic and distant to me, has a different perspective now and its beauty has broadened my understanding and expectations of science.

My family has been my rock in this journey and I am deeply grateful for their understanding and unconditional support to the best of their power and resources: to my husband, Oscar, my mother, María, my sister, Mauren, and my uncle, Luis. Regardless of all the situations we have been through, the fruits of those years of joint efforts and patience are just about to become a reality.

The members of Prof. Müller's research group have made my life in Göttingen and my doctoral project a highly rewarding experience full of many valuable professional and personal learning lessons. Special thanks to Dr. Ulrich Welling and Katrin Glormann for their time and support during my landing in this new territory and to Weihua Li, Guojie Zhang, Ludwig Schneider, and Yuliya Smirnova for the many fruitful long unexpected scientific discussions, I highly appreciated them. Marcel Langenberg, Israel Barragan, Anton Iakolev, Veronica Chiappa and Laura Endter, it has been my pleasure to exchange opinions, experiences and advise in our multiple conversations.

To my friends and beloved ones who gave me their time even when they barely had some for themselves, who procured my focus and clarity during different stages of this project as well as many joyful moments along the way: Angelica Sandoval, Alvaro Andres Argoti, Arnd-Christian Wassmus, César Beleño, Gabriel Niño, Gloria Barcenas, Julio Ríos, Marleen Triebiger, Melanie Haas, Shayan Rahimian and Vanessa Pacheco. Special thanks to Gustavo Andrés Salguero-López and Julio Rios who made my landing in this foreign territory a more heartwarming experience with their Colombian warmth.

The research leading to this dissertation would not have been possible without the financial support of the European Union under Grant agreement No. 619793 CoLiSA.MMP (Computational Lithography for Directed Self-Assembly: Materials, Models, and Processes), and the support of the Georg-August University School of Science, GAUSS. 


\section{Abstract}

The development of nanostructured materials relies on the advancement of our understanding of their properties and behavior at the molecular level. To leverage the potential of block-copolymers (BCP) as a cutting-edge technological option for industrial applications, a deeper understanding of defect formation and removal in directed self-assembly (DSA) is required. To this end, reliable, fast, and computationally-efficient modeling techniques able to capture the physics of the BCP in DSA are needed to provide experts with the ability to test design ideas and explore unique processing conditions.

Continuum models are suitable to satisfy the latter need; in this work, we have investigated to what extent these models can be used to describe the ordering process of diblock copolymers in the particular context of DSA. Among these models, the Ohta-Kawasaki free energy functional provides good agreement with SCFT calculations and particle-based simulations regarding the phase diagram of diblock copolymers, the morphology, and meta-stability of defects, and the kinetics of DSA.

Herein, we present the fluctuation mode analysis (FMA), an alternative approach to investigate local composition fluctuations within the Ohta-Kawasaki model, which is computationally less costly. The FMA allows decoupling the composition fluctuations that a given meta-stable morphology can exhibit. This feature has been exploited for the prediction of scattering patterns where specific fluctuation modes, relevant for the detection of defects via long-wavelength scattering, were identified and investigated. This finding provides the physical foundations for the design of an improved defect-metrology procedure that includes a preliminary screening of an entire wafer and the pre-classification of defects based on their light scattering footprints. 


\section{Contents}

1 Introduction $\quad 1$

1.1 Directed self-assembly . . . . . . . . . . . . . . . . . . 4

1.2 Outline of this work . . . . . . . . . . . . . 9

2 Theory and methods $\quad 11$

2.1 Continuum models for microphase separation of block copolymers 12

2.1.1 Self-consistent field theory (SCFT) . . . . . . . . . . . . . 14

2.1.2 Random-Phase-Approximation (RPA) . . . . . . . . . 15

2.1.3 Swift-Hohenberg model . . . . . . . . . . . . . 17

2.1.4 Ohta-Kawasaki model . . . . . . . . . . . . . . . 19

2.1.5 Accuracy of the continuum models in the context of DSA . 21

2.2 Detailed implementation of the Ohta-Kawasaki model . . . . . 26

2.2.1 Mapping of model parameters . . . . . . . . . . . . 26

2.2.2 Numerical implementation . . . . . . . . . . . . . . . . 28

2.3 Summary and conclusions . . . . . . . . . . . . . . . . 40

3 Kinetics of self-assembly of block copolymers 43

3.1 Defect motion and annihilation . . . . . . . . . . . . . . . . . 44

3.1.1 Dynamics of unstable defects . . . . . . . . . . . . . 44

3.1.2 Interaction of dislocations under tension . . . . . . . . 47

3.2 Understanding DSA using a continuum model . . . . . . . . . . . 50

3.2.1 Stages of directed structure formation . . . . . . . . . . . 50

3.2 .2 Spinodal length scale . . . . . . . . . . . . . . . 58

3.3 Summary and conclusions . . . . . . . . . . . . . . 67

4 Defect annihilation pathways $\quad 69$

4.1 Fluctuation Mode Analysis . . . . . . . . . . . . . . 70

4.1.1 Numerical computation of fluctuation modes . . . . . . 73

4.1.2 Fluctuation modes of defect-free structures . . . . . . . . . 81

4.1.3 Fluctuation modes of topological defects . . . . . . . . 87 
4.2 Fluctuation modes and minimum free-energy paths (MFEP) . . . . . . . . . . . . . . . . . . . . . . . . 89

4.2.1 String Method and MFEP . . . . . . . . . . . . . . . . 91

4.2.2 Numerical computation of MFEP . . . . . . . . . . . . . . . 93

4.2.3 Results and comparison . . . . . . . . . . . . . . . 98

4.3 Dominant modes in defect annihilation . . . . . . . . . . . . 104

4.4 Fluctuation modes in defect metrology in DSA . . . . . . . . . 110

5 Final remarks and Outlook $\quad 127$

$\begin{array}{ll}\text { Bibliography } & 130\end{array}$ 


\section{Chapter 1}

\section{Introduction}

Natural and synthetic polymers constitute myriads of materials necessary for daily life, thereby serving in diverse functions ranging from the DNA and the keratin in human bodies, the starch in our daily diet, and the polyacrylic acid in the superabsorbents of diapers to the aramids-aromatic polyamides used in fire-resistant materials, such as Kevlar. The study of polymers has abiding interest due to the vast variety of properties that can be tailored via their molecular design, the tunable interaction between the long polymeric chains with themselves as well as with the environment, and the diverse processing conditions available.

In the quest for smart materials with tunable novel properties, synthetic polymers span a unique exploration space whose limits are still not entirely unveiled. Polymers can have different architectures that depend on the arrangement and the type of the monomers, i.e., the repeating units forming polymeric molecules. Advancements in living polymerization techniques [1] have rendered it possible to design complex polymeric molecules, such as dendritic, hybrid hyperbranched-linear, and surface-tethered polymer brushes. Materials exhibiting diverse properties obtained with simpler polymer architectures motivated the development of such complex molecules. These simple and nowadays common molecular designs, shown in Figure 1.1, are still the focus of active research for the development of novel materials and applications.

Among the simple molecular designs, diblock copolymers can be highlighted as one of the simplest architectures. Nevertheless, they offer a rich variety of microstructures depending on the volume fraction $f$ of the A-component in the block copolymer (BCP), and the thermodynamic repulsion of the blocks quantified by the product of the Flory-Huggins parameter and the number of statistical segments per macromolecule, $\chi N$. Figure 1.2 shows the experimental phase diagram for a specific $\mathrm{BCP}$ illustrating the commonly investigated phases in this type of systems. New ordered phases in BCPs have been predicted by theory 

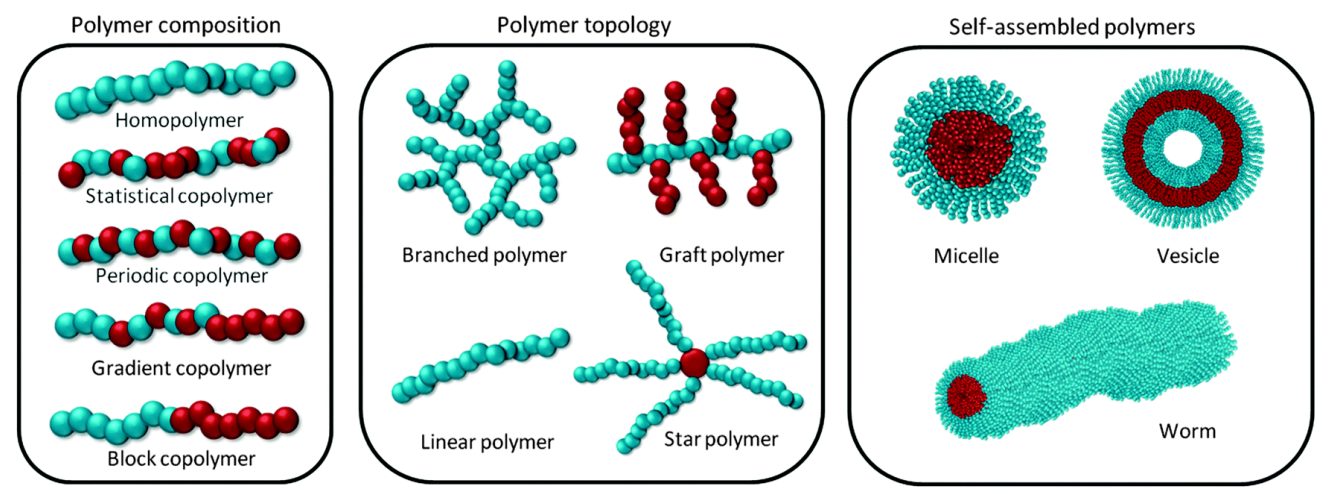

Figure 1.1: Selection of polymer architectures and self-assembled systems in solution. Reproduced from reference [2] with permission of the Royal Society of Chemistry.

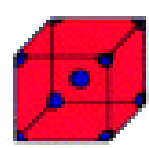

$\mathrm{S}$

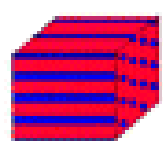

$\mathrm{C}$

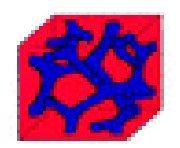

G

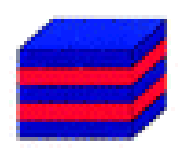

L

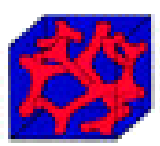

$\mathrm{G}^{\prime}$

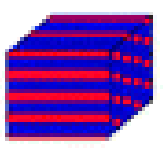

$\mathrm{C}^{\prime}$

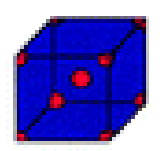

$\mathrm{S}^{\prime}$

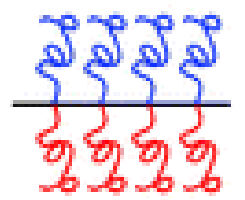

lamellar

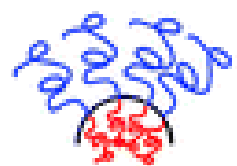

cylinder

gyroid sphere

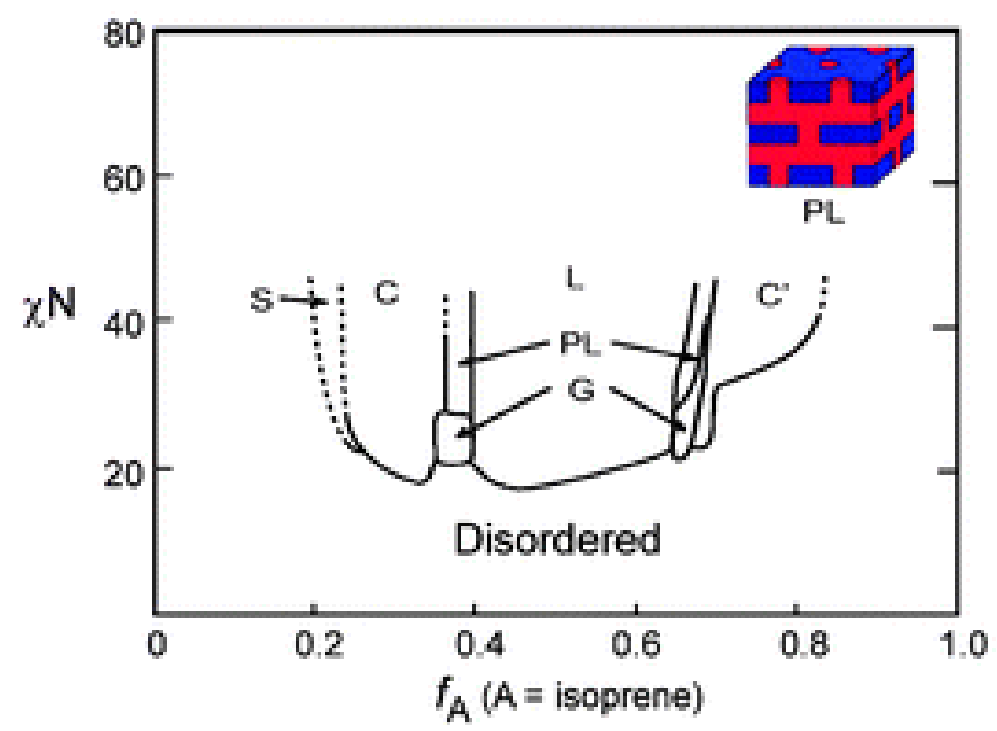

Figure 1.2: Block copolymer (BCP) microstructures and the phase diagram for the polystyrene-polyisoprene diblock copolymer system: spheres (S), cylinder $(C)$, lammela $(\mathrm{L})$, gyroid $(\mathrm{G})$, perforated lamellar (PL). Reproduced from reference [3] with permission of the Royal Society of Chemistry. 
and simulation giving rise to new fabrication processes and the engineering of functional materials, as is the case for ordered crystals of soft ellipsoids [4]

During the course of the structure formation from the disordered state to an ordered phase, defects form, merge, and annihilate as part of the self-assembly process. Despite the spontaneous annihilation of most of the defects initially formed, some will remain to be part of the final meta-stable structure where the corresponding phase, e.g., lamella, hexagonal cylinders, or others, is predicted by the phase diagram of the BCP. Therefore, defects are the result of the kinetics of the ordering process [5] rather than an equilibrium property of the polymer system. Figure 1.3 illustrates the structure formation process of a lamellar phase where defects can be observed at intermediate stages of the evolution.

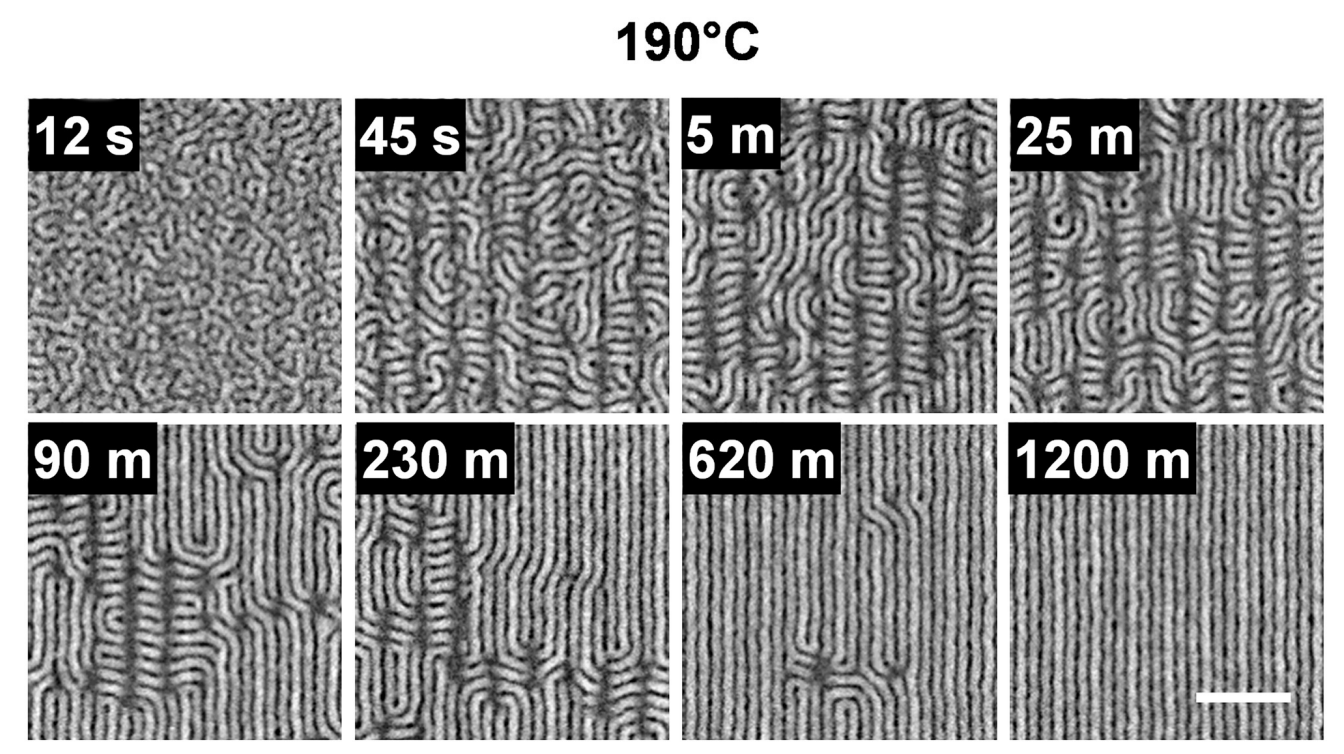

Figure 1.3: SEM images of the kinetic evolution of the guided assembly process at $190{ }^{\circ} \mathrm{C}$. The brighter domains correspond to polystyrene (PS), and the darker domains correspond to poly(methyl methacrylate) (PMMA). Defects are eventually annihilated to form fully aligned domains. Scale bar represents $200 \mathrm{~nm}$. Dark wide stripes apparent at $t=5 \mathrm{~m}$ correspond to the underlying chemical guiding pattern. Adapted with permission from reference [6]. Copyright 2018 American Chemical Society.

Defects of varying topology stem from the formation of different ordered phases that block copolymers exhibit. Among them, defects in lamellae- and cylinder-forming BCP systems are the most investigated; Figure 1.4 presents the defects that are commonly found in line patterns produced by using this type of BCP systems. 


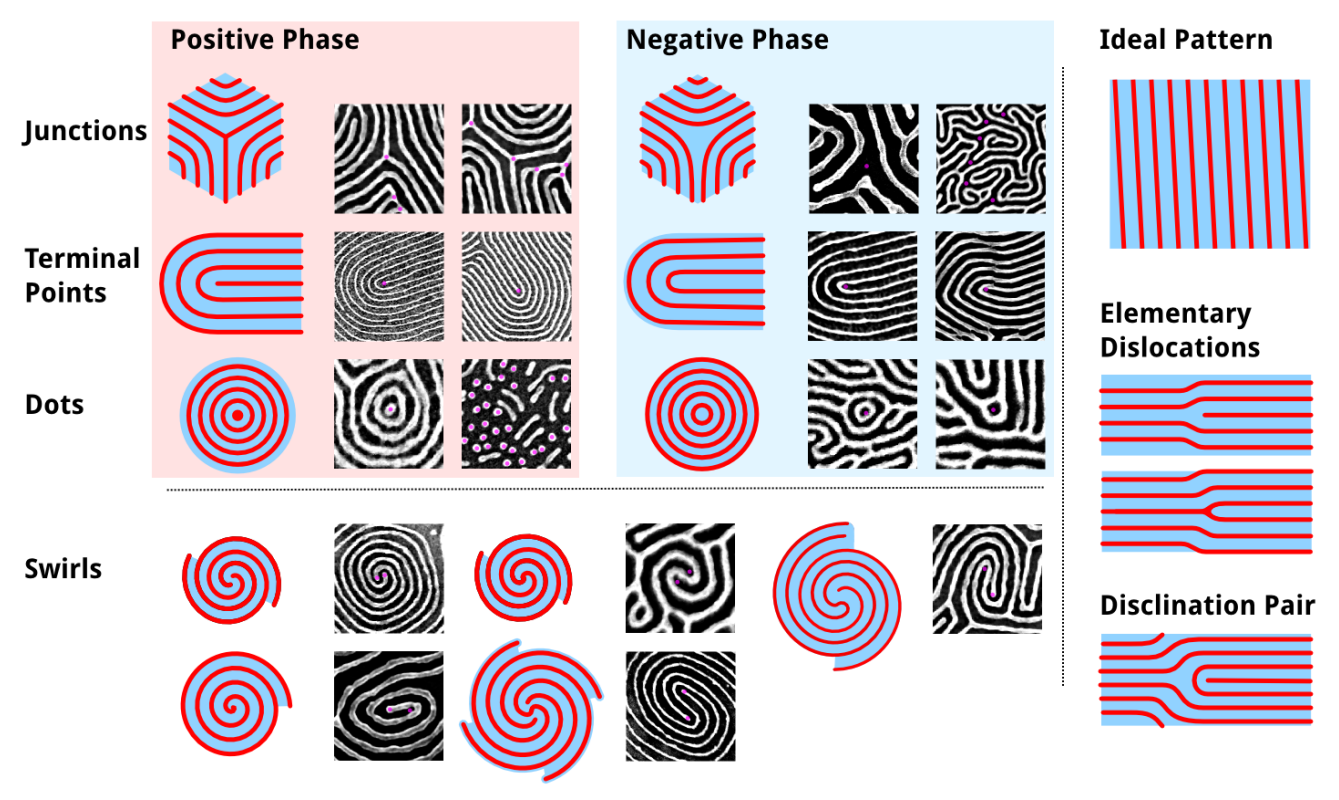

Figure 1.4: Topological defect components typically found in BCP thin film nanopatterns. Each major type of component defect is shown, as exists in either the positive (e.g. P2VP) phase or the negative (e.g. PS) phase. For each, 3-branch junctions, terminal points, and dots, examples are given with defects highlighted by a magenta dot. Reproduced from reference [7] according to the CC BY license

\subsection{Directed self-assembly}

The presence of defects in the ordered phases of BCP limits their application; thus, the use of external fields has been extensively investigated to avoid defect formation during the ordering process [8]. The deployment of short-range chemical and topological guiding fields, i.e., chemoepitaxy and graphoepitaxy respectively, has attracted increasing interest due to the possibility of using traditional lithography technology to produce the required guiding patterns for directing the self-assembly process of BCP. [5]

Hence, directed self-assembly (DSA) of copolymer materials has emerged as a promising strategy for templating dense arrays of nanostructures with extremely low defect density and spatial registration with other device components. [5, 8-23] In contrast to conventional lithography techniques used to fabricate patterned structures at the nanoscale, DSA generates the patterns in a chemical based process by exploiting the thermodynamic interactions between the domains of the block copolymer and guiding surfaces. Moreover, DSA acts as a bridge between the top-down and bottom-up lithography process. A typi- 
cal flow for DSA using chemical guiding patterns is illustrated in Figure 1.5. At the outset, the guiding pattern is imprinted by using conventional lithography and the surface modification is deployed to control the preference of the surface towards the A or B domains of the BCP or none (neutral surface). Upon finishing the fabrication of the guiding pattern, the surface is coated with the BCP usually via spin-coating techniques that allow an improved control of the film thickness. As a result, a BCP film of homogeneous local concentration of A or B domains is obtained, which corresponds to the disorder state. The micro-phase separation process is activated by using solvents or modified temperatures in a procedure known as solvent (or thermal) annealing, thus ordering the two domains of the polymer chains in the film. A final ordered structured is obtained as a result of the interplay between the design of the guiding pattern, the processing conditions and the molecular features of the polymer. These last two variables are encoded in the product $\chi N$ and the volume fraction $f$ dictating the equilibrium structure that can be expected based on the corresponding phase diagram.

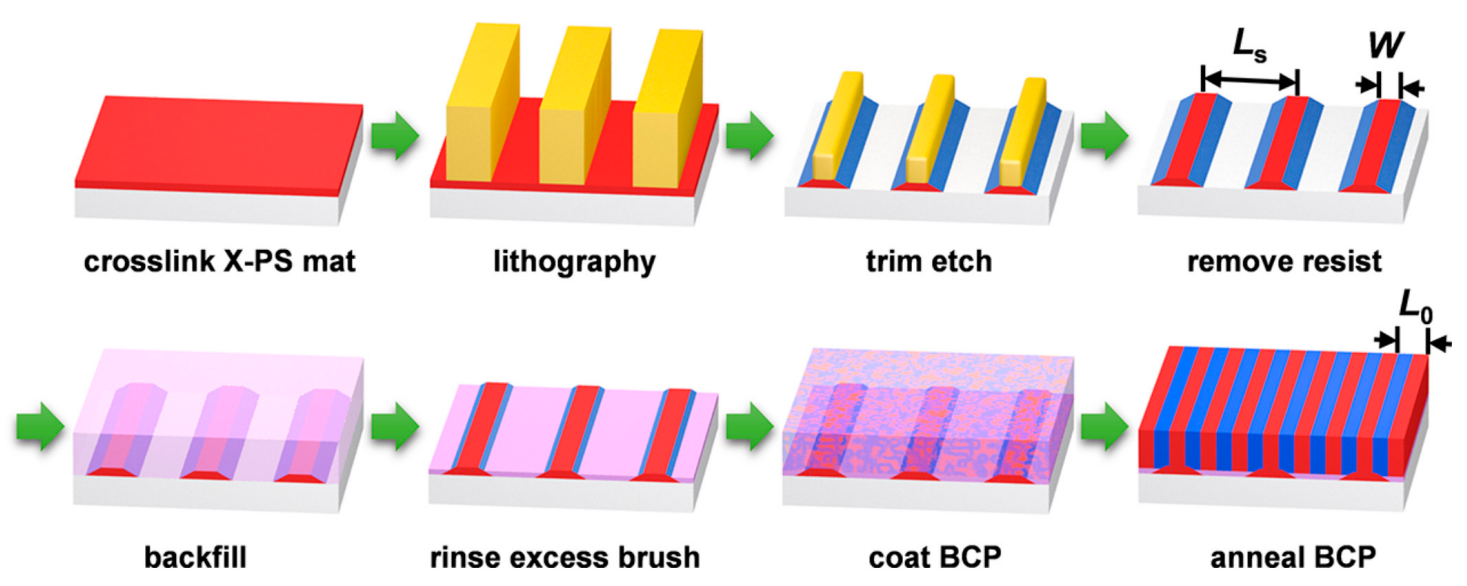

Figure 1.5: Outline of the chemical pattern formation and DSA process. The fabrication of the guiding pattern involves the modification of the surface to obtain $A-$ domains attractive stripe patterns of width $W$ located at a distance $\mathrm{L}_{s}$ from each other. The BCP is coated onto the guiding pattern and annealed to self-assemble resulting in stripes of a smaller size corresponding to the natural spacing of the polymer, $\mathrm{L}_{\mathrm{O}}$. Adapted with permission from reference [6]. Copyright 2018 American Chemical Society.

To fulfill the exacting demands of technological application, much effort has been devoted to (i) optimize the copolymer materials, (ii) tailor the guiding patterns, and (iii) devise processes that mitigate defect formation and facilitate defect annihilation. Defectivity, however, remains one of the main challenges 
delaying the extensive use of DSA in industrial applications, such as the manufacture of integrated circuits; see Figure 1.6.

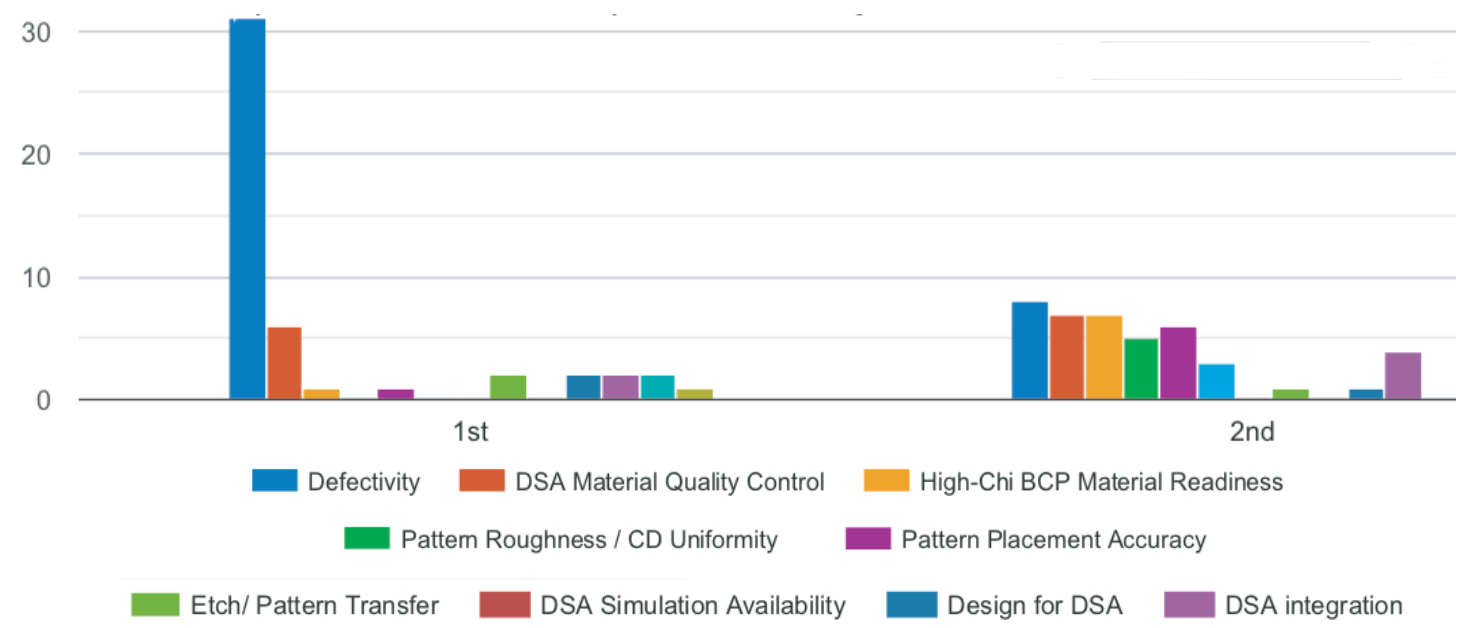

Figure 1.6: Rank of the two most critical areas for insertion of DSA for high volume integrated-circuits manufacturing. Data from DSA Survey with 48 participants at the 4th International Symposium on DSA, November 11-13, 2018, Sapporo, Japan.

The investigation of DSA is a truly multiscale problem; see Fig.1.7: AB diblock copolymers are comprised of two, thermodynamically incompatible chain molecules, $A$ and $B$, that are linked together into a linear macromolecule. The covalent bonding of the two blocks prevents macrophase separation, and thus, the molecules arrange into periodic nanostructures. The symmetry and periodicity are dictated by the balance between the volume fraction $f$ of the Acomponent, the interface free-energy between the $A$ and $B$ domains and conformational entropy loss due to stretching as the chain molecules uniformly fill space.[24-27] The interface tension depends on the details of molecular structure and interactions. The conformational entropy loss is set by the ratio $\left(L / R_{e}\right)^{2}$ between the periodicity, $\mathrm{L}$, and the polymer's end-to-end distance, $\mathrm{R}_{e}$; for Gaussian chains, $R_{e}=b \sqrt{N-1}$ where $b$ is the statistical segment length. The parameters, $\chi$ and $b$, are determined by the chemical structure of the two components, $A$ and $B$, and their prediction requires an atomistic description. In the following we assume that both blocks are characterized by the same statistical segment length, $b=b_{A}=b_{B}$. Moreover, we consider symmetric systems with $f=1 / 2$ that form lamellae in the bulk unless stated otherwise.

The mean-field description of microphase separation in the standard Gaussian chain model [29] does not involve the individual parameters, $b, N$, and $\chi$ but only the coarse-grained invariants, $R_{e}$ and $\chi N$ that quantifies the thermo- 


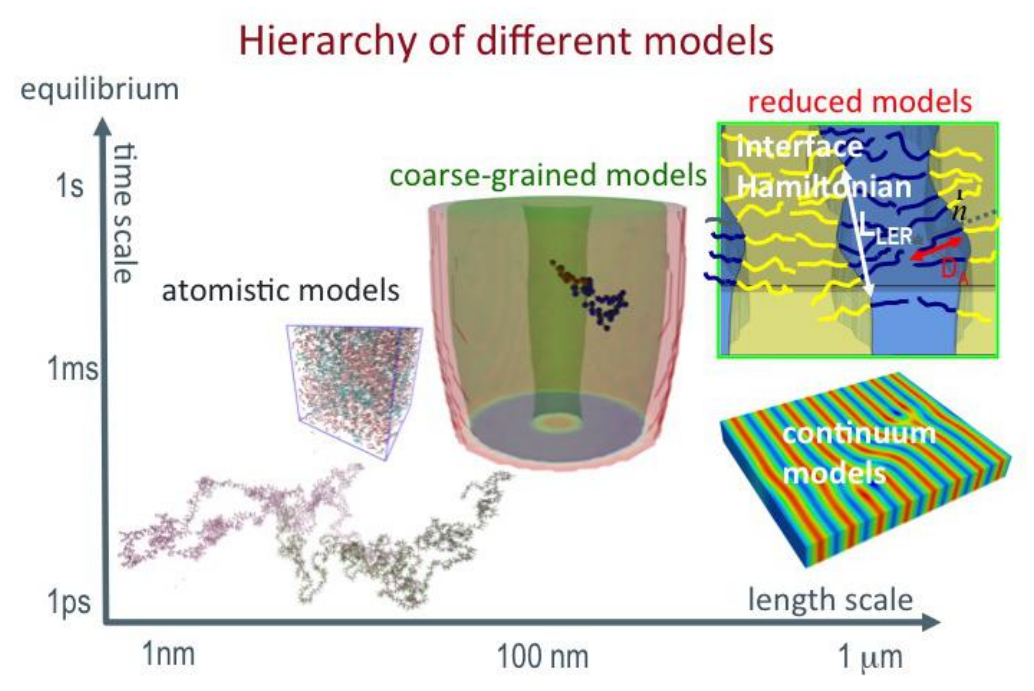

Figure 1.7: Illustration of the three levels of modeling of DSA materials. Reproduced from reference [28] with permission from the Royal Society of Chemistry.

dynamic incompatibility between the blocks, i.e., the description is invariant under changing the segmentation $\mathrm{N}$ of the molecular contour.[30-32] Fluctuation effects that give rise to line-edge roughness (LER) or line-width-roughness (LWR) in lamellar-forming copolymers,[33-37] are controlled by the invariant degree of polymerization, $\overline{\mathcal{N}}=\left(\rho \mathrm{R}_{\mathrm{e}}^{3} / \mathrm{N}\right)^{2}$, that quantifies the number of polymers a given chain molecule interacts with, where $\rho$ denotes the number density of segments. Topographical guiding patterns as well as the free surface of the copolymer film are often represented by hard, impenetrable walls because the length of the surface to the vapor, or a solid, boundary is much narrower than all other length scales of interest. The preference of surfaces towards one of the copolymer components is often parameterized by the dimensionless difference of surface tension, $\Delta \gamma \mathrm{R}_{\mathrm{e}}{ }^{2} / \sqrt{\mathcal{\mathcal { N }}} \mathrm{k}_{\mathrm{B}} \mathrm{T}$. In the standard Gaussian chain model, copolymer materials that are described by the same coarse-grained invariants, $f, R_{e}, \chi N, \overline{\mathcal{N}}$, and $\Delta \gamma$ exhibit the same behavior.[32]

This universality of copolymer behavior allows the use of highly coarsegrained models where each effective interaction center - bead - corresponds to a large number of chemical repeating units. The relevant interactions - chain connectivity, thermodynamic repulsion between unlike blocks, uniform density, and surface preference - are represented by computationally efficient potentials, e.g., a bead-spring model with harmonic bonds, soft, repulsive interactions between bead, and surface potentials whose range is a small fraction of $R_{\mathrm{e}}$. We choose the strength of these interactions to reproduce the experimental values of the coarse-grained invariants, $R_{e}, \chi N, \overline{\mathcal{N}}$, and $\Delta \gamma \mathrm{R}_{\mathrm{e}}{ }^{2} / \sqrt{\mathcal{\mathcal { N }}} k_{\mathrm{B}} \mathrm{T}$. These 
top-down coarse-grained models can be studied by self-consistent field theory or particle-based simulation.[30-32, 38]

To increase the computational speed even further, one can drop the notion of individual molecules altogether and describe the system configuration by collective variables. Two qualitatively different type of approaches have been employed: (i) interface Hamiltonians where the morphology is characterized by the spatial location of the internal $A B$ interfaces[25, 26, 33, 39, 40] and (ii) continuum free-energy functionals where the morphology is described by the local concentration of $A$ segments; see Figure 1.8. [41-56]. The former approach is very useful in the strong-segregation regime, i.e., $\chi N \gg 10$; furthermore, it has been successfully applied to study LER.[33] Recent applications of DSA, however, focus on small periodicities, $\mathrm{L}<20 \mathrm{~nm}$. This requires small $\mathrm{N}$ and a careful choice of the chemical structure - high- $\chi$ materials - to bring the invariant $\chi \mathrm{N}$ over the mean-field threshold, $\chi \mathrm{N}_{\mathrm{ODT}} \approx 10.5$. At this value, microphase separation can be observed, in other words, systems with small L are typically not in the strong-segregation regime.

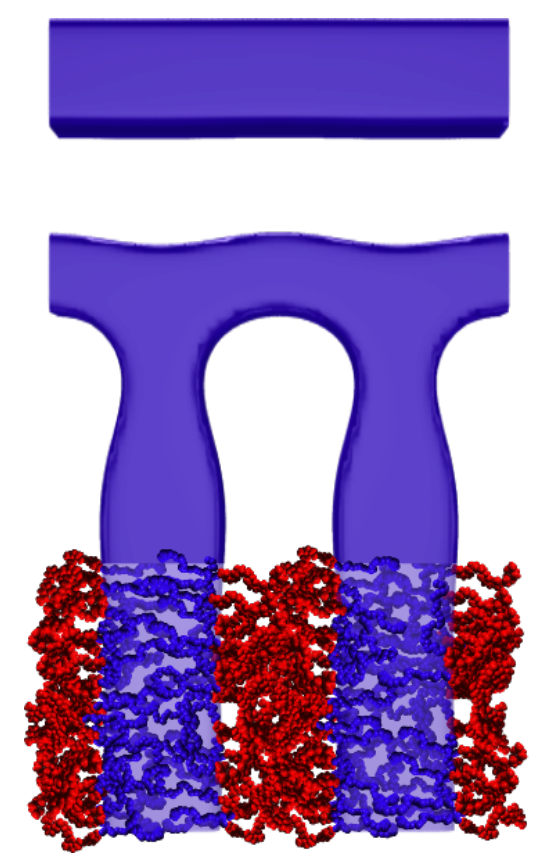

Figure 1.8: T-junctions of A-domains (blue) are obtained via computer simulations by using the Ohta-Kawasaky model free-energy functional. Schematic polymer chains depict the underlying structure of the system represented by the collective variables leading to a significant reduction in the degrees of freedom required to describe the system. Adapted from reference [28] with permission from the Royal Society of Chemistry. 
Continuum free-energy functionals provide the free energy of the system in terms of the spatially inhomogeneous, normalized densities, $\phi_{A}(\mathbf{r})$ and $\phi_{B}(\mathbf{r})$. In the technologically important weak-segregation limit, $\chi N \approx \chi N_{\text {ODT }} \approx 10.5$, the free-energy functional can be derived from the standard Gaussian chain model.[24, 42, 43, 55]

\subsection{Outline of this work}

Despite the computational advantages of continuum free-energy functional arising from the reduced degrees of freedom, the question remains about to what extent these reduced models can describe the structure formation process in the context of DSA. In this regard, this dissertation aims at answering this question, which is accomplished in the following sequence.

Chapter 2 discusses the development of continuum models from an exact theory to present the assumptions, advantages and limitations behind those models. Afterwards, we critically assess their ability to capture the equilibrium and dynamic properties pertinent to DSA by comparing them to self-consistent field theory (SCFT) and computer simulations of soft, coarse-grained particle models. [57-59] Based on these findings, we select the model that provides the most accurate description of the relevant DSA features. This chapter ends by detailing the implementation of the selected model as well as the mapping of its parameters to the coarse-grained invariants, $\chi N$ and $f$, thus enabling the quantitative comparison to results obtained with other theories and models.

Chapter 3, is concerned with the study of the kinetics of the ordering process. We investigate how the selected continuum model describes the kinetics of defect motion and annihilation under conditions that mimic the confining effect of chemical or topographical guiding patterns. Predictions for defect annihilation kinetics via the continuum model are compared to SCFT results to validate the selected model and discuss its limits. Moreover, we study how topological confinements, designed to obtain a particular non-periodic structure, affect the structure formation process in combination with the guiding effect of a chemical pattern, i.e. graphoepitaxy. Two characteristic length scales are involved in the formation process in this latter case: the initial periodicity $\lambda_{\max }$ that dictates the surface-directed spinodal ordering at early stages and the equilibrium domain spacing $\mathrm{L}_{\mathrm{O}}$ driving the final stage of the ordering process. We argue that the mismatch between those two length scales could be exploited for the design of DSA process conditions.

Given the importance of defects in DSA, we explore alternatives to predict the changes in a defective morphology at the onset of the transition pathway towards the corresponding defect-free state, which involves overcoming a free- 
energy barrier. In that regard, chapter 4 presents an alternative approach for the study of local-composition fluctuations within a continuum model: the fluctuation mode analysis (FMA). For this approach, we resort to the concept of normal modes and the study of phonons in crystalline solids to investigate small fluctuations of the local composition. The FMA provides a less computationallycostly approach to investigate fluctuations compared to solving a stochastic partial differential equation, specifically, the Cahn-Hilliard-Cook equation. After describing its foundations, we compare the predictions of the FMA to local composition changes at the onset of a transition pathway obtained via a well established method, the improved string method. Subsequently, we present the application of the FMA in the investigation of defect annihilation and for the prediction of scattering patterns. This last application aims to provide insights for developing an alternative procedure for defect detection and characterization from light-scattering data of a self-assembled structured obtained via DSA that could optimize the efforts in defect characterization by using more expensive and time-consuming techniques, such as SEM, AFM or X-ray scattering.

To conclude, chapter 5 summarizes the findings of our investigations and presents some perspectives for future work that exploit further the advantages of the continuum model as well as of the fluctuation mode analysis.

All the simulations referred to in this dissertation were performed in the CPU High Performance Computing facilities of GWDG Göttingen, the HLRN Hannover/Berlin, and the John von Neumann Center for Computing (NIC), Jülich, Germany, by using self-written programs in C with OpenMP parallelization as well as scripts in python for post-processing. Customized data visualization via self-written scripts was performed by using Matplotlib [60], ParaView [61] and Gnuplot [62]. Some 2D plots were obtained with Grace [63]. The routine $L A P A C K E$ dsyev from the Intel®Math Kernel Library is deployed for solving the eigenvalue problems in Chapter 4 using parallelization and optimization via the $C$ Intel compiler. This dissertation was typeset by the author in LaTeX.

Various materials included in the present chapter have been published as part of the special collection of papers on the subject, "Advances in Directed Self-Assembly," with the title "Continuum models for directed self-assembly" in the journal Molecular Systems Design \& Engineering. The content has been adapted and reproduced from Ref. [28] with permission from the Royal Society of Chemistry. 


\section{Chapter 2}

\section{Theory and methods}

This chapter is devoted to the description of the theoretical aspects pertaining to the continuum models. We discuss how the Ohta-Kawasaky and the SwiftHohenberg free energy functionals are derived from self-consistent field theory (SCFT) as an accurate theory to depict the thermodynamics of block copolymer (BCP) structures. We focus on how these two free-energy functionals can describe the meta-stability and morphology of defects as key features for structures obtained via DSA. A comparison of the predictions for these features via the continuum models and SCFT is performed to highlight the model that provides the more accurate representation. For that selected model, a mapping of the model parameters to the coarse-grained invariant is presented. In the final section, additional details for the numerical implementation of our calculations are included.

The materials presented in this chapter have been published as part of the special collection of papers on the subject, "Advances in Directed Self-Assembly," with the title "Continuum models for directed self-assembly" in the journal Molecular Systems Design \& Engineering. To facilitate the integration of the papers' contents into this dissertation, only the sections of the paper directly related with the scope of this chapter have been included herein, specifically, sections 2, 3.1, 3.2, 3.3 and 4. Part of the content of those sections has been omitted in this chapter and it is indicated by [...], in order to avoid duplication with other chapters in this dissertation.

In integrating the paper's content, the cross-references, citations, and figure numbers used in the paper have been adjusted to be consistent with those in this dissertation. The content has been adapted and reproduced from Ref. [28] with permission from the Royal Society of Chemistry. Additional details of the numerical calculations not included in the published paper are presented in section 2.2.2. 


\subsection{Continuum models for microphase separation of block copolymers}

Continuum models [41-56] strike a compromise between retaining some aspects of the predictive power of more detailed molecular models and enabling a computationally screening of the multi-dimensional parameter space and the reverse design of the DSA process specified by (i) the copolymer materials, (ii) the geometry and interactions of the guiding pattern, and (iii) the process conditions that control the kinetics of DSA.

Instead of using the molecular degrees of freedom, i.e., positions and momenta of polymer segments, as fundamental descriptors of a microscopic configuration, continuum models operate on a coarser scale and employ a collective order-parameter, $\mathrm{m}$. Since a dense $A B$ copolymer melt is nearly incompressible $\phi_{\mathrm{A}}(\mathbf{r})+\phi_{\mathrm{B}}(\mathbf{r})=1$, one often describes the two-component system by a single, spatially varying order parameter, $m(\mathbf{r}) \sim \phi_{A}(\mathbf{r})-\phi_{B}(\mathbf{r})$, that quantifies the difference in the local density of the two components, $A$ and B. Typically the order-parameter field, $m(\mathbf{r})$, is evaluated on a collocation grid, whose spatial discretization is set by the smallest relevant length scale, i.e., the width of the internal $A B$ interfaces. Thus the values of the order parameter at the grid vertices characterize a configuration, giving rise to a drastic reduction in the number of degrees of freedom in comparison to a molecular description. This choice of the order-parameter is common to both continuum models discussed in the following, and it imposes several limitations from the outset:

- Since the use of a single collective order parameter ignores which molecule and which part of it contributes to the density at position $\mathbf{r}$, the quantitative description of architectural effects (e.g., polydispersity, chain-end effects, or homopolymer-copolymer mixtures) is limited.

- The assumption of incompressibility obviously breaks down at the narrow surface of the polymer film to the supporting substrate or the free surface to air/vacuum. More generally, the appropriate formulation of boundary conditions is challenging because the changes of the molecular conformations at a sharp inhomogeneity are not explicitly described.

- Like in dynamic SCFT, one assumes that the chain configurations are always in equilibrium with the instantaneous order-parameter and often uses approximates to relate the dynamics of the interacting molecules to the order-parameter kinetics.

Having defined the order parameter, continuum models seek to describe the equilibrium thermodynamics by a free-energy functional, $\mathcal{F}[\mathrm{m}]$. Two different 
continuum models are discussed in literature - (i) the Swift-Hohenberg model or phase-field crystal model [41-48] and (ii) the Ohta-Kawasaki free-energy functional [49-56] - to model the structure and thermodynamics of spatially modulated phases.

In principle, $\mathcal{F}[\mathrm{m}]$, can be constructed by comparison to SCFT in the weaksegregation limit (RPA-mapping) [24, 42, 43, 55] or simulations of particle-based models in conjunction with field-theoretic umbrella sampling.[32, 64] In practice, however, phenomenological expressions are employed that represent expansions in terms of powers of the order parameter and its spatial derivatives supplemented by general symmetry considerations and that match the fluctuation spectrum of a copolymer melt in the disordered state. These symmetry considerations specify the free-energy functional in the bulk, and $\mathcal{F}[\mathrm{m}]$ is augmented by terms that describe the interaction with the guiding pattern and boundary conditions. The minima of $\mathcal{F}[\mathrm{m}]$ specify the equilibrium morphologies and allow for the construction of the equilibrium phase diagram of confined copolymer systems.

The relation between the dynamics of the individual macromolecules in the course of the ordering process and the collective kinetics of structure formation, i.e., the time-evolution of $m(r, t)$ is routinely parameterized by an Onsager coefficient, $\Lambda$. This Onsager coefficient quantifies the order-parameter current, $\mathbf{j}(\mathbf{r})$ that is generated by a gradient of the chemical potential, $\mu(\mathbf{r})=\delta \mathcal{F} / \delta \mathrm{m}(\mathbf{r})$. Formal expressions for $\Lambda$ can be derived, e.g., $\Lambda \sim \phi_{A}(\mathbf{r}) \phi_{B}(\mathbf{r})$ to account for incompressibility or $\Lambda \sim \mathrm{g}\left(\mathbf{r}, \mathbf{r}^{\prime}\right)$ where $\mathrm{g}$ denotes the intramolecular correlation function, but computing $\Lambda$ is a formidable task.[65-67] Routinely, drastic approximations, e.g., ignorance of composition-dependence and non-locality of $\Lambda$, are employed, resulting in:

$$
\mathbf{j}(\mathbf{r})=-\Lambda \nabla \mu(\mathbf{r})
$$

This current and the time evolution of the order parameter are connected via the continuity equation, which the conservation of the species $A$ and $B$ also imparts onto $m$, resulting in a conserved Cahn-Hilliard dynamics for the order parameter (model B according to the classification of dynamic universality classes according to Hohenberg and Halperin [68]).

$$
\frac{\partial \mathrm{m}}{\partial \mathrm{t}}=-\nabla \mathbf{j}=\Lambda \triangle \frac{\delta \mathcal{F}}{\delta \mathrm{m}(\mathbf{r})}
$$

In this simple form, $\Lambda$ is a constant and can be adsorbed as a scale factor of time. Different numerical schemes have been devised to integrate this partial differential equation in time. In our exploratory study we use the simple forward Euler scheme. 


\subsubsection{Self-consistent field theory (SCFT)}

In the limit of large $\overline{\mathcal{N}}$, the thermodynamics of block copolymer structures can be accurately described by self-consistent field theory (SCFT).[69-73] This approach has been successfully used to study the phase diagram,[27, 29] defect properties $[5,23,59]$ and the minimum free-energy path of defect annihilation.[57, 74] Within SCFT the statistical mechanics of interacting macromolecules is approximated by the properties of non-interacting chain molecules in self-consistently determined, external fields, $w_{A}(\mathbf{r})$ and $w_{B}(\mathbf{r})$, acting on the $A$ blocks and $B$ blocks, respectively. Given $w_{A}(\mathbf{r})$ and $w_{B}(\mathbf{r})$, the most probable densities of non-interacting Gaussian chain molecules subjected to the external fields,

$$
\phi_{A}^{*}(\mathbf{r})=-\mathrm{V} \frac{\delta \ln Q\left[w_{A}^{*}, w_{B}^{*}\right]}{\delta w_{A}(\mathbf{r})}
$$

and likewise for $\phi_{\mathrm{B}}^{*}(\mathbf{r})$, can be computed via a modified diffusion equation.[6973] Here $Q\left[w_{A}^{*}, w_{B}^{*}\right]$ denotes the single-chain partition function of a diblock copolymer in volume $V$ subjected to the external fields, $w_{A}$ and $w_{B}$. Inverting this relation, one obtains the external fields, $w_{A}^{*}[\Psi]$ and $w_{B}^{*}[\Psi]$, that correspond to a given order parameter, $\Psi(\mathbf{r})=\phi_{A}(\mathbf{r})-f$ and satisfy the incompressibility constraint, $\phi_{\mathrm{A}}(\mathbf{r})+\phi_{\mathrm{B}}(\mathbf{r})=1$. SCFT yields the following estimate for the free-energy functional

$$
\begin{aligned}
\frac{\mathcal{F}[\Psi]}{k_{B} T}= & \frac{\rho}{N} \int \mathrm{d} \mathbf{r}\left(\chi N\left\{f[1-f]+[1-2 f] \Psi-\Psi^{2}\right\}\right. \\
& \left.-w_{A}^{*}[\Psi+f]-w_{B}^{*}[1-f-\Psi]\right) \\
& -\sqrt{\overline{\mathcal{N}}} \frac{V}{R_{e}{ }^{3}} \ln \mathcal{L}\left[w_{A}^{*}, w_{B}^{*}\right]
\end{aligned}
$$

Whereas this free-energy functional is accurate, it is computationally much more demanding than the continuum models discussed in the following because it requires the solution of the inverse problem for $w_{A}^{*}[\Psi]$ and $w_{B}^{*}[\Psi]$.

Here we use the spectral approach [72] to compute the equilibrium properties of lamellae formed by symmetric diblock copolymers, $f=0.5$, that serve as accurate reference data to gauge the accuracy of the continuum models. Minimizing the free-energy functional, Eq. (2.4), yields the self-consistency condition for the order parameter

$$
w_{A}^{*}\left[\Psi^{*}\right]-w_{B}^{*}\left[\Psi^{*}\right]=1-2 f-2 \Psi^{*}
$$

that dictates the order parameter, $\Psi^{*}(\mathbf{r})$, in the thermodynamic equilibrium. Back-substituting $\Psi^{*}(\mathbf{r})$ into Eq. (2.4), we obtain the free energy, $\mathrm{F}=\mathcal{F}\left[\Psi^{*}\right]$ and, minimizing the equilibrium free energy, $F$, with respect to the periodicity, we estimate the equilibrium domain spacing, $\mathrm{L}_{0}$. 


\subsubsection{Random-Phase-Approximation (RPA)}

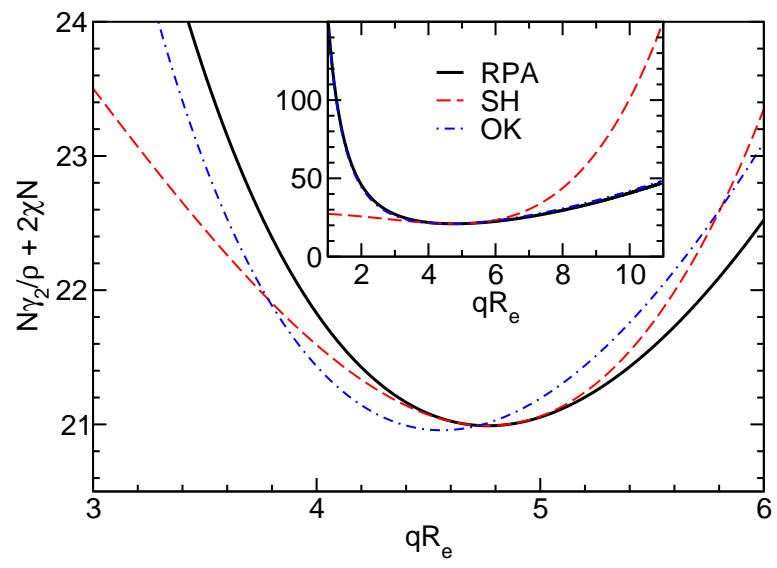

Figure 2.1: Comparison of the second-order vertex function obtained from the random-phase approximation (RPA) with the approximations employed in the Swift-Hohenberg (SH) model and the Ohta-Kawasaki (OK) model.

In principle, an accurate free-energy functional, $\mathcal{F}$, can be numerically obtained by SCFT. If the local A density $\phi_{A}(\mathbf{r})$ only deviates slightly from its average $f$, one can systematically expand the free-energy in terms of the order parameter, $\Psi(\mathbf{r})=\phi_{A}(\mathbf{r})-\mathrm{f}$. This Random-Phase-Approximation (RPA) yields for the free-energy difference with respect to the homogeneous state, $\Psi(\mathbf{r})=0,[24,42,43,55]$

$$
\begin{aligned}
\frac{\mathcal{F}[\Psi]}{k_{\mathrm{B}} T}= & +\frac{1}{2 !} \int \frac{\mathrm{d} \mathbf{q}}{(2 \pi)^{3}} \gamma_{2}(\mathbf{q}) \Psi_{\mathbf{q}} \Psi_{-\mathbf{q}} \\
& +\frac{1}{3 !} \int \frac{\mathrm{d} \mathbf{q}_{1} \mathrm{~d} \mathbf{q}_{2}}{(2 \pi)^{6}} \gamma_{3}\left(\mathbf{q}_{1}, \mathbf{q}_{2}\right) \Psi_{\mathbf{q}_{1}} \Psi_{\mathbf{q}_{2}} \Psi_{-\mathbf{q}_{1}-\mathbf{q}_{2}} \\
& +\frac{1}{4 !} \int \frac{\mathrm{d} \mathbf{q}_{1} \mathrm{~d} \mathbf{q}_{2} \mathrm{~d} \mathbf{q}_{3}}{(2 \pi)^{9}} \gamma_{4}\left(\mathbf{q}_{1}, \mathbf{q}_{2}, \mathbf{q}_{3}\right) \Psi_{\mathbf{q}_{1}} \Psi_{\mathbf{q}_{2}} \Psi_{\mathbf{q}_{3}} \Psi_{-\mathbf{q}_{1}-\mathbf{q}_{2}-\mathbf{q}_{3}} \\
& +\cdots
\end{aligned}
$$

where $\Psi_{\mathbf{q}}=\int \mathrm{d} \mathbf{r} \exp (\mathbf{i q r}) \Psi(\mathbf{r})$ is the Fourier transform of the order parameter. Both continuum models are based on this fourth-order expansion, Eq. (2.6). The RPA of Leibler [24] provides explicit expressions for the wavevector-dependent vertex functions, $\gamma_{i}$. Both continuum models neglect the wavevector dependence of the vertex functions $\gamma_{3}$ and $\gamma_{4}$. For the symmetric case, one obtains 
$v_{0}=\frac{\mathrm{N}}{\rho} \gamma_{3}=0$ and $\mathrm{u}_{0}=\frac{\mathrm{N}}{\rho} \gamma_{4} \approx 156.56$. Within mean-field approximation, this second-order vertex function is directly related to the collective structure factor in the disordered phase, $\frac{1}{S(q)} \sim \gamma_{2}(q)$, and takes the explicit form

$$
\begin{aligned}
\frac{N}{\rho} \gamma_{2}(\mathbf{q})= & F(x, f)-2 x N \quad \text { with } \quad x=\frac{\left(q_{\mathrm{e}}\right)^{2}}{6} \\
F(x, f)= & \frac{g(x, 1)}{g(x, f) g(x, 1-f)-\frac{1}{4}[g(x, 1)-g(x, f)-g(x, 1-f)]^{2}} \\
\text { and } \quad & g(x, f)=\frac{2}{x^{2}}\left[e^{-f x}-1+f x\right]
\end{aligned}
$$

The function $F(x, f)$ describes the influence of the chain conformations on the collective composition fluctuations, and $g(x, f)$ is the Debye function that describes the Gaussian conformational statistics of an individual block. For a symmetric block copolymer, the vertex function exhibits a minimum at $x^{*} \approx 3.7852$ or $L=2 \pi / \mathrm{q}^{*} \approx 1.32 \mathrm{R}_{\mathrm{e}}$ and adopts the value

$$
\frac{\mathrm{N}}{\rho} \gamma_{2}\left(\mathrm{q}^{*}\right)=\tau_{0} \approx 2(10.495-\chi \mathrm{N})
$$

The two continuum models differ in the approximation used for the secondorder vertex function. The Swift-Hohenberg model approximates $\gamma_{2}(q)$ by a parabola around $q^{*}$

$$
\frac{N}{\rho} \gamma_{2-S H}(q)=\tau_{0}+\epsilon_{0}\left[\left(q^{*} R_{e}\right)^{2}-\left(q R_{e}\right)^{2}\right]^{2} \quad \text { with } \quad \epsilon_{0}=\frac{1}{74.81}
$$

The Ohta-Kawasaki model [49] additionally captures the asymptotic behavior for small and large wavevectors

$$
\frac{\mathrm{N}}{\rho} \gamma_{2-\mathrm{OK}}(\mathrm{q})=\frac{(\mathrm{qR})^{2}}{3}+7.1-2 \chi \mathrm{N}+\frac{144}{(\mathrm{qR})^{2}}
$$

The three forms of the wavevector-dependent second-order vertex functions are depicted in Fig. 2.1. The Swift-Hohenberg model provides an excellent approximation in the ultimate vicinity of the minimum but yields only a poor representation of the vertex function for large and small wavevectors. The OhtaKawasaki model, in turn, accurate captures the asymptotic behavior of the vertex function for $q \rightarrow 0$ and $q \rightarrow \infty$, as well as the value of the vertex function at its minimum, but it slightly underestimates the position of the minimum, $\mathrm{q}_{\mathrm{OK}}^{*} \mathrm{R}_{\mathrm{e}}=2 \sqrt[4]{3^{3}} \approx 0.957 \mathrm{q}^{*} \mathrm{R}_{\mathrm{e}}$ 


\subsubsection{Swift-Hohenberg model}

Using the parabolic approximation of the vertex function, one obtains the SwiftHohenberg model for symmetric block copolymers:[41-48]

$$
\begin{gathered}
\frac{\mathcal{F}[\Psi]}{k_{\mathrm{B}} \mathrm{T}}=\frac{\rho}{\mathrm{N}} \int \mathrm{d} \mathbf{r}\left(\frac{1}{2} \Psi\left\{\tau_{0}+\epsilon_{0}\left[\left(\mathrm{q}^{*} \mathrm{R}_{\mathrm{e}}\right)^{2}-\left(i \nabla \mathrm{R}_{\mathrm{e}}\right)^{2}\right]^{2}\right\} \Psi\right. \\
\left.+\frac{\mathrm{u}_{0}}{4 !} \Psi^{4}\right)
\end{gathered}
$$

In the following, we introduce units of length $\xi_{0}$, energy $\eta_{0}$, and order parameter $\Psi_{0}$, and define the dimensionless, rescaled variables

$$
\mathbf{x}=\frac{\mathbf{r}}{\xi_{0}} \quad \text { and } \quad \mathcal{F}_{\mathrm{SH}}[\mathrm{m}]=\frac{\mathcal{F}[\Psi]}{\eta_{0}} \quad \text { with } \quad \mathrm{m}=\frac{\Psi}{\Psi_{0}}
$$

Relating the units of the continuum models to the coarse-grained invariants via

$$
\begin{aligned}
\xi_{0} & =\frac{1}{\mathrm{q}^{*}} \approx 0.21 \mathrm{R}_{\mathrm{e}} \\
\Psi_{0} & =\sqrt{\frac{6}{\mathrm{u}_{0}} \epsilon_{0}\left(\mathrm{q}^{*} \mathrm{R}_{\mathrm{e}}\right)^{4}} \approx 0.514 \\
\eta_{0} & =\frac{\rho}{\mathrm{N}} \mathrm{k}_{\mathrm{B}} T \xi_{0}^{3} \Psi_{0}^{2} \epsilon_{0}\left(\mathrm{q}^{*} \mathrm{R}_{\mathrm{e}}\right)^{4} \\
& =\mathrm{k}_{\mathrm{B}} T \sqrt{\overline{\mathcal{N}}} \Psi_{0}^{2} \epsilon_{0}\left(\mathrm{q}^{*} \mathrm{R}_{\mathrm{e}}\right) \approx 0.0168 \mathrm{k}_{\mathrm{B}} T \sqrt{\overline{\mathcal{N}}}
\end{aligned}
$$

we arrive at the standard form of the Swift-Hohenberg free-energy functional

$$
\begin{aligned}
\mathcal{F}_{\mathrm{SH}}[\mathrm{m}] & =\int \mathrm{d} \mathbf{x}\left(\frac{1}{2} \mathrm{~m}\left\{-\tilde{\epsilon}+[1+\triangle]^{2}\right\} \mathrm{m}+\frac{1}{4} \mathrm{~m}^{4}\right) \\
& =\int \mathrm{d} \mathbf{x}\left(-\frac{\tilde{\epsilon}}{2} \mathrm{~m}^{2}+\frac{1}{4} \mathrm{~m}^{4}+\frac{1}{2}\{[1+\triangle] \mathrm{m}\}^{2}\right)
\end{aligned}
$$

where the spatial derivatives are taken with respect to the rescaled, dimensionless coordinate, $\mathbf{x}$. The qualitative behavior in the rescaled units only depends on the parameter

$$
\tilde{\epsilon}=-\frac{\tau_{0}}{\epsilon_{0}\left(q^{*} R_{e}\right)^{4}} \approx 0.29(\chi N-10.495)
$$

The Swift-Hohenberg free-energy functional captures the minimal ingredients that are relevant for describing spatially modulated phases. It has been employed to study the universal aspects of structure formation in systems with 
different underlying interactions (Rayleigh-Bénard convection, magnetic garnets, Turing patterns, lipid membranes, diblock copolymers).[46] The model has also attracted recent interest under the name of phase field crystal (PFC) model,[45] and it has also been studied in the case that the spatial average of the order parameter, $\overline{\mathrm{m}}$, does not vanish.

From Eq. (2.17) we calculate the chemical potential and the equation of motion

$$
\begin{aligned}
\mu(\mathbf{r})=\frac{\delta \mathcal{F}_{S H}}{\delta m(\mathbf{r})} & =-\tilde{\epsilon} m+m^{3}+[1+\triangle]^{2} m \\
\frac{\partial m}{\partial t} & =\nabla \wedge \nabla\left(-\tilde{\epsilon} m+m^{3}+[1+\triangle]^{2} m\right)
\end{aligned}
$$

The Swift-Hohenberg model can reproduce the qualitative phase behavior of block copolymers in the weak segregation limit and it is the computationally simplest form to describe spatially modulated phases. The local character of the free-energy functional enables a rather straightforward computation of the free energy. These attractive properties have made the model rather popular for studying the universal, qualitative features of spatially modulated phases and the kinetics of structure formation.

The parameter $\tilde{e}$ dictates the equilibrium properties. The disordered structure, $m(\mathbf{r})=\bar{m}$, becomes unstable for $\tilde{\epsilon}>3 \bar{m}^{2}$, i.e., for a symmetric diblock copolymer with $\overline{\mathrm{m}}=0$, for $\tilde{e}>0$. For a symmetric bulk system, $\overline{\mathrm{m}}=0$, in the weak-segregation limit $0<\tilde{\epsilon} \ll 1$, the order-parameter profile can be described by a single Fourier component (one-mode approximation),

$$
\mathrm{m}_{\mathrm{SH}-\mathrm{OMA}}(\mathrm{x})=\sqrt{\frac{4 \tilde{\epsilon}}{3}} \sin (\mathrm{x})
$$

and the free-energy density in a volume $\mathrm{V}$ is $\mathrm{F}_{\mathrm{SH}-\mathrm{OMA}}=-\tilde{\epsilon}^{2} \mathrm{~V} /\left(6 \xi^{3}\right)$.

The phase diagram of the Swift-Hohenberg model exhibits a tricritical point [48] at $\tilde{\epsilon}_{\text {tri }}=9 / 38$ and $\bar{m}_{\text {tri }}=\sqrt{3 / 38}$. Beyond this tricritical point, it predicts a macrophase coexistence between a spatially modulated phase and a homogeneous phase separated by a miscibility gap. Obviously such phase coexistence cannot occur in a pure block copolymer melt. Thus the model can qualitatively describe pure diblock copolymers only for small $\tilde{e}$ and compositional asymmetries $|\bar{m}|$.

From the computational perspective, the Swift-Hohenberg model is the simplest free-energy model. The main difficulty is the high-order spatial derivative in the kinetic equation that enforces a rather small time step, $\Delta t \sim \Delta x^{6}$, for simple integration schemes to remain numerically stable. In our numerical performance test, we studied the kinetics of structure formation from a disordered 
to a lamellar structure at very weak segregation, $\epsilon=0.02$, and rather coarse spatial discretization, $\Delta x=0.3140$, with a time step of $\Delta t=0.0003$.

\subsubsection{Ohta-Kawasaki model}

Using the asymptotically exact approximation, Eq. (2.10), of the second-order vertex function, one derives the Ohta-Kawasaki model for a symmetric block copolymer

$$
\begin{aligned}
\frac{\mathcal{F}[\Psi]}{\mathrm{k}_{\mathrm{B}} \mathrm{T}}=\frac{\rho}{\mathrm{N}} \int \mathrm{d} \mathbf{r}\left(\frac{1}{2} \Psi\right. & \left\{\frac{\left(i \nabla \mathrm{R}_{\mathrm{e}}\right)^{2}}{3}+7.1-2 \chi \mathrm{N}\right. \\
& \left.\left.+\frac{144}{\mathrm{R}_{\mathrm{e}}{ }^{2}} \int \mathrm{d} \mathbf{r}^{\prime} \mathrm{G}\left(\mathbf{r}, \mathbf{r}^{\prime}\right)\right\} \Psi+\frac{\mathrm{u}_{0}}{4 !} \Psi^{4}\right)
\end{aligned}
$$

The long-range kernel, $G\left(\mathbf{r}, \mathbf{r}^{\prime}\right)$, obeys the Poisson equation

$$
-\triangle \mathrm{G}\left(\mathbf{r}, \mathbf{r}^{\prime}\right)=\delta\left(\mathbf{r}-\mathbf{r}^{\prime}\right)+\text { const }
$$

G decays with distance $r$ like $1 / r$ in three spatial dimensions, $G \sim \ln (r)$ in two dimensions, and $G\left(x, x^{\prime}\right)=-\frac{1}{2}\left|x-x^{\prime}\right|+\frac{1}{2 L}\left(x-x^{\prime}\right)^{2}$ for a one-dimensional variation of the order parameter, $m(x)$, with period $L$.

Relating the units of length, order parameter, and energy, defined in Eq. (2.12), to the coarse-grained invariants according to

$$
\begin{aligned}
\xi_{0} & =\frac{R_{e}}{\sqrt{3(2 \chi N-7.1)}} \\
\Psi_{0} & =\sqrt{\frac{6}{u_{0}}(2 \chi N-7.1)} \\
\eta_{0} & =\frac{\rho}{N} k_{B} T \xi_{0}^{3} \Psi_{0}^{2}(2 \chi N-7.1) \\
& =\frac{2 \sqrt{\mathcal{N}} k_{B} T}{\sqrt{3} u_{0}} \sqrt{2 \chi N-7.1} \approx \frac{\sqrt{2 \chi N-7.1}}{135.58} \sqrt{\overline{\mathcal{N}}} k_{B} T
\end{aligned}
$$

we arrive at the standard form of the Ohta-Kawasaki free-energy functional

$$
\begin{aligned}
& \mathcal{F}_{\mathrm{OK}}[\mathrm{m}]=\int \mathrm{d} \mathbf{x}\left(\frac{1}{2} \mathrm{~m}\left\{-\Delta-1+\tilde{\alpha} \int \mathrm{d} \mathbf{r}^{\prime} \mathrm{G}\left(\mathbf{x}, \mathbf{x}^{\prime}\right)\right\} \mathrm{m}\right. \\
&\left.+\frac{\tilde{\gamma}}{3} \mathrm{~m}^{3}+\frac{1}{4} \mathrm{~m}^{4}\right) \\
&=\int \mathrm{d} \mathbf{x}\left(-\frac{1}{2} \mathrm{~m}^{2}+\frac{\tilde{\gamma}}{3} \mathrm{~m}^{3}+\frac{1}{4} \mathrm{~m}^{4}+\frac{1}{2}[\nabla \mathrm{m}]^{2}\right) \\
&+\frac{\tilde{\alpha}}{2} \iint \mathrm{d} \mathbf{x} \mathrm{d} \mathbf{x}^{\prime} \mathrm{m}(\mathbf{x}) \mathrm{G}\left(\mathbf{x}, \mathbf{x}^{\prime}\right) \mathrm{m}\left(\mathbf{x}^{\prime}\right)
\end{aligned}
$$


where, for completeness, we have also restored the third-order term that is only required for asymmetric copolymers. The qualitative behavior of the model depends on the two parameters

$$
\begin{aligned}
& \tilde{\alpha}=\frac{144}{2 \times \mathrm{N}-7.1} \frac{\xi_{0}^{2}}{\mathrm{R}_{\mathrm{e}}^{2}}=\frac{48}{(2 \times \mathrm{N}-7.1)^{2}} \\
& \tilde{\gamma}=0 \quad \text { for symmetric copolymers }
\end{aligned}
$$

The calculation of the free energy of a configuration, $m(\mathbf{r})$, can be conveniently performed in Fourier space, where the convolution with the long-range kernel, G, reduces to a multiplication. The model also predicts hexagonal and spherical phases, and it does not suffer from the spurious macrophase separation of the Swift-Hohenberg model.

From Eq. (2.28) we calculate the chemical potential and the equation of motion

$$
\begin{aligned}
\frac{\delta \mathcal{F}_{\mathrm{OK}}}{\delta \mathrm{m}(\mathbf{r})} & =-\mathrm{m}+\tilde{\gamma} \mathrm{m}^{2}+\mathrm{m}^{3}-\Delta \mathrm{m}+\alpha \int \mathrm{d} \mathbf{x} \mathrm{G}\left(\mathbf{x}, \mathbf{x}^{\prime}\right) \mathrm{m}\left(\mathbf{x}^{\prime}\right) \\
\frac{\partial \mathrm{m}}{\partial \wedge \mathrm{t}} & =\Delta\left(-\mathrm{m}+\gamma \mathrm{m}^{2}+\mathrm{m}^{3}-\triangle \mathrm{m}\right)-\alpha \mathrm{m}
\end{aligned}
$$

The use of a local Onsager coefficient and the property, Eq. (2.23), of the nonlocal kernel, G, gives rise to a computationally convenient form of the timeevolution equation that only involves fourth-order spatial derivatives.

For a symmetric bulk system, $\tilde{\gamma}=0$, in the weak-segregation limit $\frac{1}{4}=$ $\tilde{\alpha}_{\text {ODT }}>\tilde{\alpha} \gg 0$, the order-parameter profile can be described by a single Fourier component (single-mode approximation), $\mathrm{m}_{\mathrm{OK}-\mathrm{OMA}}(x)=\mathrm{m}_{0} \sin \left(2 \pi x / \mathrm{L}_{0}\right)$, with amplitude and periodicity

$$
\mathrm{m}_{0}^{2}=\frac{4}{3}(1-2 \sqrt{\tilde{\alpha}}) \quad \text { and } \quad \frac{\mathrm{L}_{0}}{\xi_{0}}=\frac{2 \pi}{\tilde{\alpha}^{1 / 4}}
$$

and the free-energy density is $\mathrm{F}_{\mathrm{OK}-\mathrm{OMA}}=-(1-2 \sqrt{\tilde{\alpha}})^{2} \mathrm{~V} /\left(6 \xi_{0}^{3}\right)$.

In the opposite limit of strong segregation, $\tilde{\alpha} \ll \frac{1}{4}$, one can approximate the order-parameter profile by a sequence of wide, alternating domains that are separated by narrow $A B$ interfaces. The profile of an individual $A B$ interface is obtained by optimizing the local and square-gradient contribution to the free-energy functional. This yields the same profile as the corresponding homopolymer interface, i.e., $\operatorname{moK}_{\mathrm{OSSL}}(x)=\tanh (x / \sqrt{2})$ (in rescaled units).[49] The interfaces are characterized by a tension of $\sigma \xi_{0}^{2}=\frac{2 \sqrt{2}}{3}$. To evaluate the long-range contribution, one approximates the interface profile by a sharp-kink 
profile

$$
m(x)=\sum_{k=1}^{\infty} \frac{4(-1)^{k}}{\pi(2 k+1)} \cos \left(\frac{2 \pi}{L_{0}}(2 k+1) x\right)
$$

Optimizing the total free energy with respect to the lamellar periodicity, $\mathrm{L}_{0}$, one obtains $[49,55,56]$

$$
\frac{\mathrm{L}_{0}}{\xi_{0}}=\frac{4 \sqrt[6]{2}}{\tilde{\alpha}^{1 / 3}} \quad \text { and } \quad \frac{\mathrm{F}_{\mathrm{OK}-\mathrm{SSL}}}{\mathrm{V} / \xi_{0}^{3}}=-\frac{1}{4}+\left(\frac{\tilde{\alpha}}{4}\right)^{1 / 3}
$$

\subsubsection{Accuracy of the continuum models in the context of DSA}

In the following we consider symmetric diblock copolymers, $f=1 / 2$ (i.e., $\tilde{\gamma}=$ 0 ), and compare the description of the two continuum models with the prediction of SCFT.

\section{Free energy and domain spacing}

In the vicinity of the order-disorder transition (ODT) the RPA-mapping between the coarse-grained invariants and the parameters of the continuum models is supposed to be accurate. A first, crude estimate of the range of validity of the RPA-mapping can be obtained by requiring that $|\Psi|<f=1 / 2$. Using Eqs. (2.14) and (2.21) for the Swift-Hohenberg model, we find that $\tilde{\epsilon}<\frac{3}{16 \Psi_{0}^{2}} \approx 0.71$. Using Eq. (2.18) we obtain the condition $\chi N<12.9$. By the same token, Eqs. (2.25) and (2.33) also yield the condition $\chi \mathrm{N}<\mathrm{u}_{0} / 64+3.55+\sqrt{48} \approx 12.9$ for the Ohta-Kawasaki model.

In Fig. 2.2 we quantitatively compare the free-energy difference per copolymer molecule between the lamellar and disordered phases obtained by the two continuum models and SCFT.

$$
f \equiv \frac{\Delta F}{n k_{B} T}=\frac{F}{\frac{V}{R_{e}{ }^{3}} k_{B} T \sqrt{\overline{\mathcal{N}}}}=\frac{F_{O K}}{V / \xi_{0}^{3}} \cdot \frac{\eta_{0}}{k_{B} T \sqrt{\mathcal{N}}} \cdot\left(\frac{R_{e}}{\xi_{0}}\right)^{3}
$$

In the inset of the figure we demonstrate that in the ultimate vicinity of the ODT, indeed, the predictions of the two continuum models in conjunction with the RPA mapping quantitatively agree. The free-energy difference quadratically depends on the distance, $\chi \mathrm{N}-10.5$ from the ODT because the transition is of second-order within the mean-field treatment. As expected from the rational above, the agreement significantly deteriorates for incompatibilities larger than $\chi N=12.9$; this limit is indicated by the vertical dashed line. 


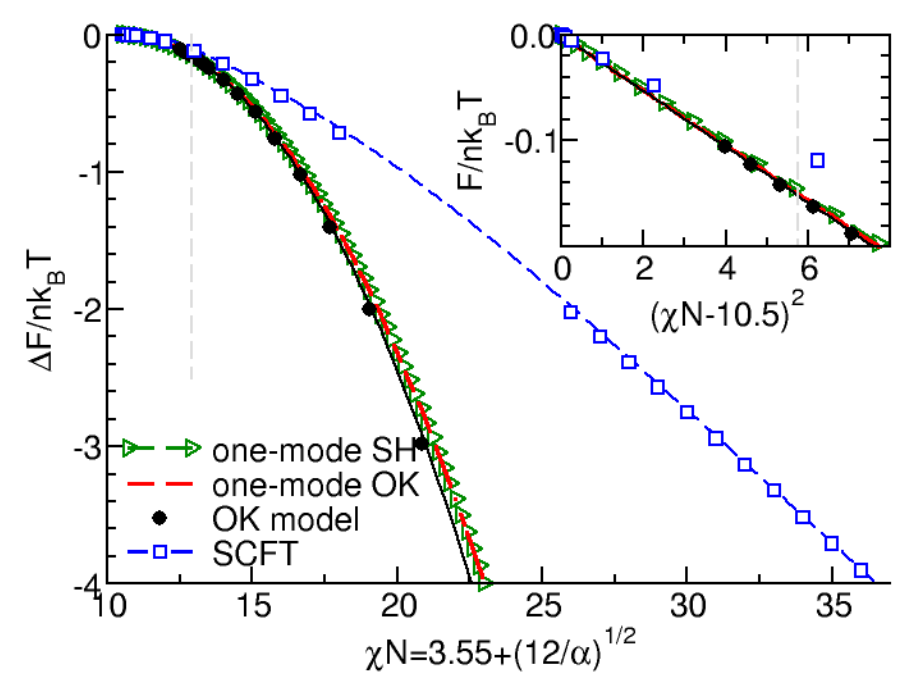

Figure 2.2: Comparison of the free energy per chain in units of $k_{B} T$ obtained by the Swift-Hohenberg model (in one-mode approximation), by the OhtaKawasaki model, and SCFT. The vertical dashed line indicates the validity limit of the RPA-mapping, $\chi N=12.9$. The inset presents the behavior in the ultimate vicinity of the order-disorder transition (ODT).

In Fig. 2.3 we compare the preferred lamellar spacing in units of the unperturbed end-to-end distance, $R_{e}$.

$$
\frac{\mathrm{L}_{0}}{\mathrm{R}_{\mathrm{e}}}=\frac{\mathrm{L}_{0}}{\xi_{0}} \cdot \frac{\xi_{0}}{\mathrm{R}_{\mathrm{e}}}
$$

Within the one-mode approximation of the Swift-Hohenberg model, the lamellar spacing does not vary with incompatibility and, by construction, agrees with the SCFT value at the ODT. The Ohta-Kawasaki model, in turn, slightly overestimates the lamellar spacing at the onset of ordering because the location of the minimum of $\gamma_{2}$ is underestimated (cf. Fig. 2.1). Within the one-mode approximation we obtain from Eqs. (2.35) and (2.24) also a $\chi \mathrm{N}$-independent lamellar period, $\frac{L_{0}}{R_{\mathrm{e}}}=\frac{2 \pi}{\tilde{\alpha}^{1 / 4}} \times \frac{\xi_{0}}{R_{\mathrm{e}}}=\pi 3^{-3 / 4}$. Beyond the one-mode approximation, the lamellar spacing increases but the comparison with the SCFT calculation reveals that the dependence of $\mathrm{L}_{0}$ on incompatibility is significantly different already at intermediate values of $\chi \mathrm{N}$.

From this comparison in Figs. 2.2 and 2.3, we conclude that both continuum models in conjunction with the RPA-mapping provide a quantitative description of lamella-forming diblock copolymers in the ultimate vicinity of the ODT, 


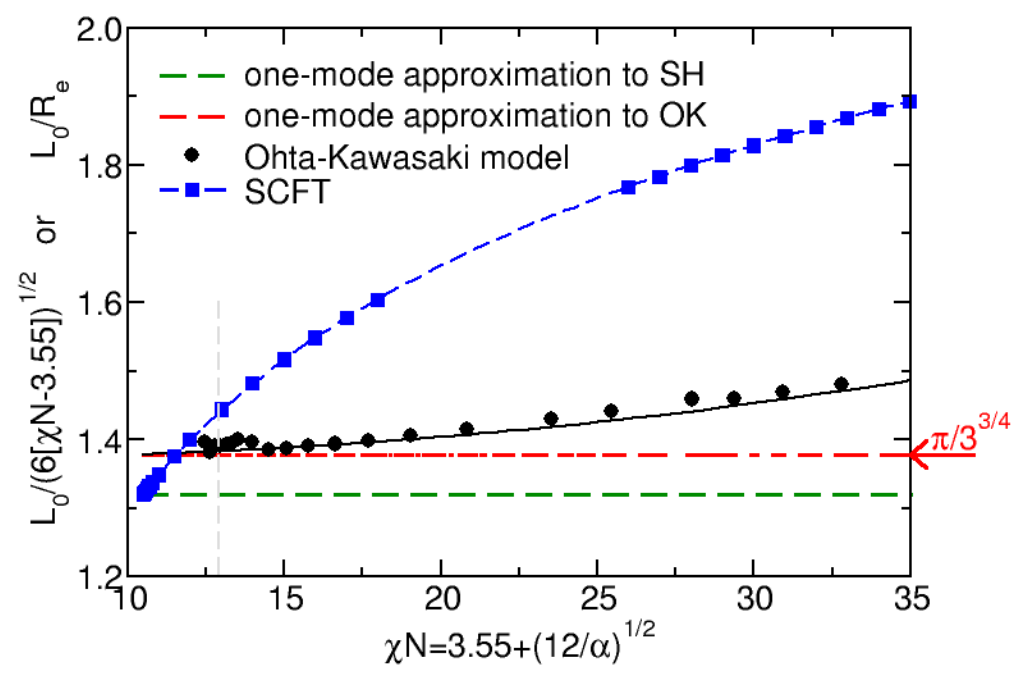

Figure 2.3: Comparison of the equilibrium lamellar spacing, $\mathrm{L}_{0}$ obtained by the Swift-Hohenberg model, the Ohta-Kawasaki model, and SCFT. The vertical dashed line indicates the validity limit of the RPA-mapping, $\chi \mathrm{N}=12.9$.

$\mathrm{XN}<12.9$, but fail to predict the free energy and domain spacing at intermediate and large segregation strengths.

Although novel high- $\chi$ materials, which are intensely explored to fabricate structures with the smallest feature sizes, are characterized by small $\mathrm{N}$ (and therefore also small $\chi \mathrm{N}$ ), this ultimate vicinity of the ODT is only of limited practical use because the high- $\chi$ materials are also characterized by small values of the invariant degree of polymerization, $\overline{\mathcal{N}}$. Thus fluctuation effects become important. One hallmark of fluctuations is the shift of the ODT [42] to larger values of incompatibility, $\chi \mathrm{N}_{\mathrm{ODT}} \approx 10.495+\frac{41}{\mathcal{N}^{-1 / 3}}$. Thus, for a polymer system with $\overline{\mathcal{N}}<5000$ the ODT is shifted to values larger than $\chi \mathrm{N}=12.9$, and there is no interval of incompatibilities whatsoever, where any of the two continuum models in conjunction with the RPA-mapping provides an accurate, quantitative description. Using generic values of $1 \mathrm{~g} / \mathrm{cm}^{3}$ for the density of the melt, and a statistical segment of mass $100 \mathrm{~g} / \mathrm{mol}$ and length $b=0.7 \mathrm{~nm}$, we extract the number density, $\rho$, of segments and obtain $\sqrt{\bar{N}}=\rho b^{3} \sqrt{N} \approx 2.07 \cdot \sqrt{N}$, i.e., a polymer with $\overline{\mathcal{N}}<5000$ is comprised of less than 1167 segments and is characterized by $\mathrm{R}_{\mathrm{e}}<23.9 \mathrm{~nm}$ or $\mathrm{L}_{0}<31.5 \mathrm{~nm}$. 


\section{Metastability of defects}

The previous section demonstrated that the two continuum models in conjunction with the RPA-mapping quantitatively describe the self-assembly of block copolymers only in the ultimate vicinity of the ODT, $\chi \mathrm{N}<12.9$. Here we explore whether the two models are able to capture the qualitative behavior at intermediate and larger segregation.

First, we note that the Swift-Hohenberg model for asymmetric composition predicts a macroscopic phase separation between a disordered phase and a spatially modulated one that differ in their compositions.[48] Such a macroscopic phase separation is obviously impossible in diblock melt because the chain connectivity prevents macroscopic fluctuations of the order parameter. The $1 / \mathrm{q}^{2}$ term in the Ohta-Kawasaki model, in turn, does not allow for macroscopic fluctuations of the order parameter, i.e., the model can only exhibit spatially modulated phases.

Second, SCFT calculations [57] and molecular simulations [58, 59, 75] have demonstrated that tight pairs of dislocations are only stable above a threshold of incompatibility, $\chi \mathrm{N}^{*} \approx 18$. For smaller incompatibilities, these prototypical defect pairs spontaneously annihilate - providing an important strategy for defect-free DSA.[57] We have investigated the metastability of a tight dislocation pair in the Swift-Hohenberg model and the Ohta-Kawasaki model. In the Swift-Hohenberg model, tight dislocation pairs are metastable for $\tilde{\epsilon} \geqslant 0.5$, which corresponds to $\chi \mathrm{N} \approx 12.2$ according to the RPA mapping, Eq. (2.18). For values of $\tilde{\epsilon} \leqslant 0.4$, the defect is unstable. In the Ohta-Kawasaki model, in turn, the tight dislocation pair becomes unstable for $\tilde{\alpha} \geqslant 0.80$. Using the RPA-mapping, the model parameter corresponds to $\chi N=15.8$, in rather good agreement with SCFT.

Third, we investigate the ability of continuum models to capture the geometry of the defect morphology. Fig. 2.4 presents the local density of A-segments, $\phi_{A}(x)$, of a metastable, tight dislocation pair in the lamellar phase of a symmetric diblock copolymer, obtained by SCFT calculations (a) and the corresponding equivalent morphology obtained as a local minimum of the free-energy functional of the Ohta-Kawasaki and Swift-Hohenberg models. From the orderparameter profiles of the morphology in continuum models, the location of the $A B$ interfaces can be extracted, and we focus on these interface positions in order to compare the description of the geometry within the models.

In the region between the two edge dislocations, the Swift-Hohenberg model predicts a wider lamella between the defect cores and a larger distance D between them (cf. blue contour lines in panel b of Fig. 2.4). The low accuracy in the description of the morphology by the Swift-Hohenberg model is corroborated by the comparison of the interface profiles far away from the defects, 
(a)

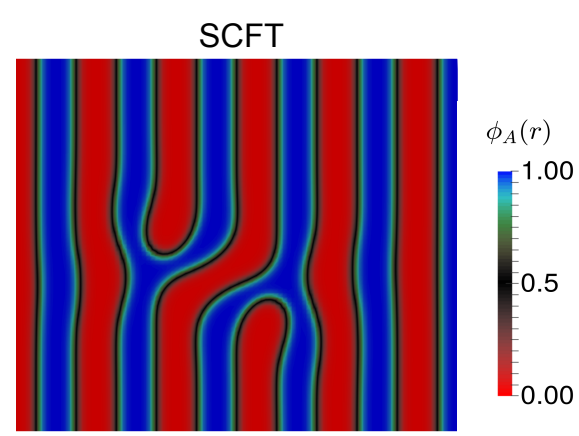

(b) Ohta-Kawasaki / SCFT

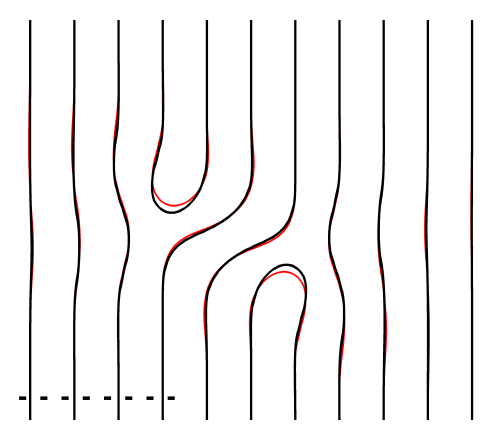

Swift-Hohenberg / SCFT

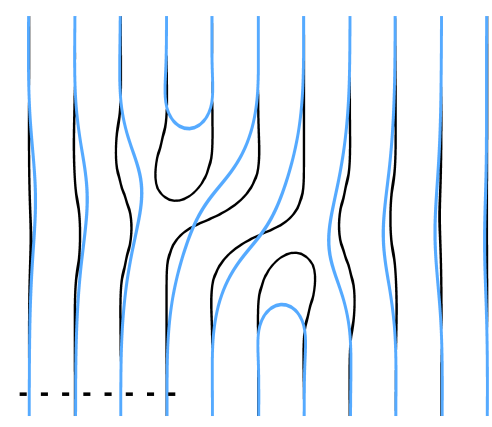

(c)
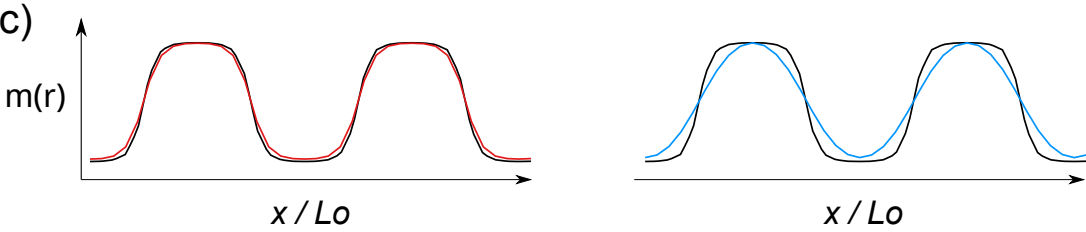

Figure 2.4: Comparison of a tight dislocation defect described by SCFT calculations with morphologies obtained within continuum models. (a) The color map represents the local density of A-segments obtained in SCFT at $\chi N=30$ and $f=1 / 2$, from which the location of the $A B$ interfaces is extracted (black lines). (b) Comparison of the location of the $A B$ interfaces as obtained by the Ohta-Kawasaki model (red lines) and Swift-Hohenberg model (blue) with the SCFT results. Parameters for continuum models $\tilde{\alpha}=0.0201$ and $\tilde{\epsilon}=5.65$, respectively, correspond to $\chi N \approx 30$. (c) Composition profile across an internal $A B$ interface along the dashed line depicted in the morphologies in panel (b). The order-parameter scale is adjusted such that the maxima and minima of the profiles coincide with the SCFT values, \pm 1 .

where the model predicts a significantly wider interface with respect to SCFT calculations. Due to the prediction of a spurious macrophase separation and the qualitative failure to capture the morphology of prototypical defects, we focus 
the following discussion on the Ohta-Kawasaki model.

The results of the Ohta-Kawasaki model, in contrast, agree significantly better with the geometry of the defect predicted by SCFT. Additionally, the composition profiles across a lamella are much better reproduced, when measuring the length scales in units of the preferred lamellar spacing and adjusting the range of the order parameter.

Previously, we have hypothesized that the metastability of defects at intermediate segregation is related to the separation of the intrinsic width, $w$, of the internal $A B$ interfaces between the domains and the periodicity, $\mathrm{L}_{0}$.[57] SCFT at strong segregation, $\chi \mathrm{N} \gg 10$, predicts $\frac{w}{\mathrm{R}_{\mathrm{e}}} \approx \frac{1}{\sqrt{6 \times \mathrm{N}}}$ and $\frac{\mathrm{L}_{0}}{\mathrm{R}_{\mathrm{e}}} \approx 2\left(\frac{8}{3 \pi^{4}}\right)^{1 / 6}(\chi \mathrm{N})^{1 / 6} \approx$ $1.1(\chi N)^{1 / 6}$, i.e., the ratio of the two lengths scales like $\frac{w}{L_{0}} \sim(\chi N)^{-2 / 3}$. In the Ohta-Kawasaki model, we obtain $\frac{w}{\xi_{0}}=\sqrt{2}$, i.e., independent from $\tilde{\alpha}$, whereas the periodicity increases like $\frac{\mathrm{L}_{0}}{\xi_{0}} \sim \tilde{\alpha}^{-1 / 3}$ for $\tilde{\alpha} \rightarrow 0$. Thus, the ratio, $\frac{w}{\mathrm{~L}_{0}} \sim \tilde{\alpha}^{1 / 3}$, of the two length scales of the lamellar profile decreases for strong segregation, $\tilde{\alpha} \rightarrow 0$, and the Ohta-Kawasaki model qualitatively captures the length-scale separation of the SCFT.

\subsection{Detailed implementation of the Ohta-Kawasaki model}

\subsubsection{Mapping of model parameters}

The failure of the Ohta-Kawasaki model in conjunction with the RPA-mapping to quantitatively predict the behavior of block copolymers at intermediate and strong segregations is not unexpected because the description is based on the truncation of the systematic expansion, Eq. (2.7), and additional approximations of the wavevector-dependence of the vertex functions. In order to systematically extend the validity of the continuum models, we have to include higher-order terms in the expansion. Whereas several authors have improved the local term in the free-energy functional, to the best of our knowledge, the wavevector-dependence of higher-order vertex functions have not been considered. Such wavevector-dependent vertex functions, however, would result in a rather complex model in real-space representation, and much of the computational benefit of the continuum models (compared to SCFT or Single-Chain-inMean-Field simulations) would be sacrificed.

Therefore, motivated by the universality of self-assembly phenomena and the ability of the Ohta-Kawasaki models to qualitatively capture some of the salient features of block-copolymer self-assembly, we will follow a different 
strategy: Rather than modifying the functional form of the free-energy functional, we merely adjust the mapping of the length scale, $\xi_{0}$, order-parameter scale, $\Psi_{0}$, and the free-energy scale, $\eta_{0}$. Thus, we replace the RPA-mapping between the scales, $\xi_{0}, \Psi_{0}, \eta_{0}$, and parameters, $\tilde{\alpha}, \tilde{\gamma}$ of the continuum model by a phenomenological relation.

The amplitude, $\Psi_{0}$, of the order parameter at intermediate and strong segregation is chosen so that $\Psi_{0} \max _{\mathbf{x}}|\mathrm{m}(\mathbf{x})|=f=\frac{1}{2}$. Rather than obtaining the scale factors for the free energy and length scales from the RPA-mapping, we use the data in Figs. 2.2 and 2.3 in conjunction with Eqs. (2.36) and (2.37). By construction, this procedure eliminates the deviations in Figs. 2.2 and 2.3, i.e., the free-energy difference, $\Delta \mathrm{F}$, between the lamellar phase and the disordered structure and the lamellar periodicity, $\mathrm{L}_{0}$, agrees with the results of the SCFT of the molecular model.

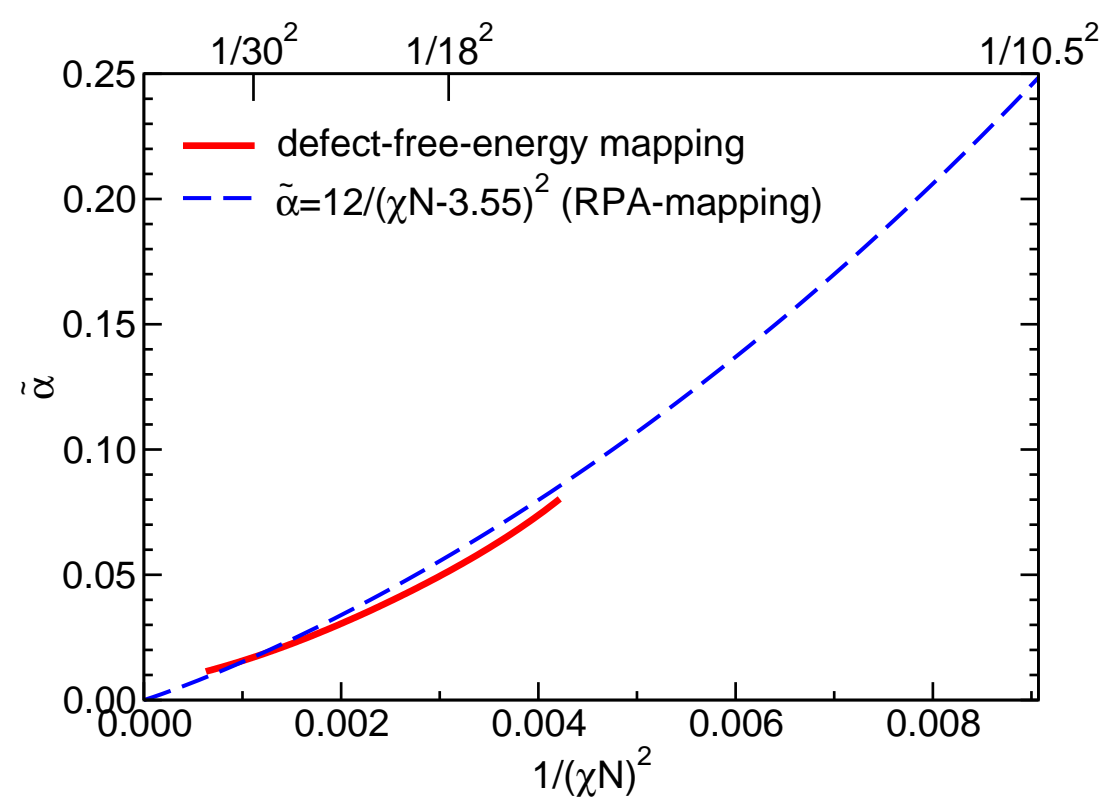

Figure 2.5: Phenomenological extension of the mapping, $\tilde{\alpha}(\chi N)$, as to reproduce the excess free energy of a metastable tight dislocation pair. The result is compared with the RPA-mapping, Eq. (2.29)

By virtue of symmetry, we expect that $\tilde{\gamma}=0$ for lamella-forming diblock copolymers with $f=\frac{1}{2}$. In the context of DSA, we choose to adjust the value of $\tilde{\alpha}$ so that the phenomenological extension of the mapping captures the excess free energy of a tight dislocation pair. To this end, we have numerically adjusted $\alpha(\chi)$ and the result of this procedure is shown in Fig. 2.5. 
We observe rather good agreement with Eq. (2.29), suggesting that the adhoc rescaling of the free-energy and length scales does not give rise to significant artifacts. Thus, we conclude that the Ohta-Kawasaki model in conjunction with the parameter identification provides an appropriate description of DSA of symmetric block copolymers.

To obtain a qualitative understanding of this phenomenological extension, we consider the naive extrapolation of the RPA-mapping to large $\chi \mathrm{N}$ or small $\tilde{\alpha}$, respectively. Eq. (2.29) and Eq. (2.24) suggest the leading-order behavior $\tilde{\alpha} \approx 12(\chi N)^{-2}$ and $\frac{\xi_{0}}{R_{\mathrm{e}}} \approx(6 \times \mathrm{N})^{-1 / 2}$. Using these extrapolations, we find $\frac{w}{\mathrm{R}_{\mathrm{e}}} \approx$ $\sqrt{2} \frac{\xi}{R_{\mathrm{e}}} \approx \frac{1}{\sqrt{3 \chi \mathrm{N}}}$ and $\frac{\mathrm{L}_{0}}{\mathrm{R}_{\mathrm{e}}} \approx \frac{4 \sqrt[6]{2}}{\alpha^{1 / 3}} \frac{\xi}{\mathrm{R}_{\mathrm{e}}} \approx 2 \cdot 3^{-5 / 6}(\chi \mathrm{N})^{1 / 6} \approx 0.8(\chi \mathrm{N})^{1 / 6}$, i.e., the qualitative asymptotic scaling behavior of the width of the intrinsic interface and the domain spacing in the strong segregation limit are reproduced by the OhtaKawasaki model (in conjunction with the extrapolated RPA-mapping). Thus, the phenomenological extension changes the prefactors but not the leading $\chi N$ dependence of the length scale, $\xi_{0}$, and the model parameter, $\tilde{\alpha}$.

In the strong-segregation limit, the maximal values of the order parameter, $\max _{\mathbf{x}}|\mathrm{m}(\mathbf{x})|$, are $\mathcal{O}(1)$, independent of $\chi \mathrm{N}$. Thus, in contrast to Eq. (2.25), the scale factor $\Psi_{0}$ will adopt a $\chi \mathrm{N}$-independent value at large segregation. This scaling behavior will also affect the scale of the free energy. Using Eq. (2.26) in conjunction with $\Psi_{0} \sim 1$ and $\xi_{0} / R_{e} \sim(\chi N)^{-1 / 2}$, we obtain $\eta_{0} / k_{B} T \sqrt{\overline{\mathcal{N}}} \sim$ $(\chi N)^{-1 / 2}$. Using these modified scale factors and the asymptotic behavior of the Ohta-Kawasaki model in the strong-segregation limit, we obtain for the $A B$ interface tension, $\sigma$, and the excess free energy, $\Delta f_{\text {lam }}$, of a copolymer in the lamellar phase, $\frac{\sigma \mathrm{R}_{\mathrm{e}}{ }^{2}}{\mathrm{k}_{\mathrm{B}} \mathrm{T} \sqrt{\mathcal{N}}}=\frac{2 \sqrt{2}}{3} \cdot \frac{\eta_{0}}{\mathrm{k}_{\mathrm{B}} \mathrm{T} \sqrt{\mathcal{N}}} \cdot\left(\frac{\mathrm{R}_{\mathrm{e}}}{\bar{\xi}_{0}}\right)^{2} \sim \sqrt{\chi \mathrm{N}}$ and $\Delta \mathrm{f}_{\text {lam }}=\left(\frac{\tilde{\alpha}}{4}\right)^{-1 / 3}$. $\frac{\eta_{0}}{k_{B} T \sqrt{\mathcal{N}}} \cdot\left(\frac{R_{e}}{\xi_{0}}\right)^{3} \sim \sqrt[3]{\chi N}$, which agree with the limit of SCFT for large $\chi N$, i.e., $\frac{\sigma \mathrm{R}_{\mathrm{e}}^{2}}{\mathrm{k}_{\mathrm{B}} \mathrm{T} \sqrt{\overline{\mathcal{N}}}} \approx \sqrt{\chi \mathrm{N} / 6}$ and $\Delta \mathrm{f}_{\text {lam }} \approx \frac{1}{4} \sqrt[3]{9 \pi^{2} \chi \mathrm{N}}$, respectively.[25, 69]

\subsubsection{Numerical implementation}

Once we have understood how the Ohta-Kawasaki model was derived, we devote this section to describe the numerical approximations and assumptions behind the computational implementation of the standard form of the free energy functional $\mathcal{F}[\mathrm{m}(\mathbf{x})]$. Once we have understood how the Ohta-Kawasaki model was derived, we devote this section to describe the numerical approximations and assumptions behind the computational implementation of the standard form of the free energy functional $\mathcal{F}[\mathrm{m}(\mathbf{x})]$.

It is important to recall at this point that the units of length, order parameter and energy in $\mathcal{F}[\mathrm{m}(\mathbf{x})]$ have been previously rescaled with respect to the coarse- 
grained invariants such that the free energy functional and its parameters are dimensionless.

To facilitate consistency checks during the implementation and leave room for the use of different coordinate systems, the Ohta-Kawasaki free energy functional is treated as three separate terms accounting for the contribution of local order parameter, the energetic cost of forming an interface and the long-range interactions kernel - the Greens function:

$$
\begin{aligned}
\mathcal{F}[\mathrm{m}(\mathbf{x})] & =\mathcal{F}^{\mathrm{L}}[\mathrm{m}]+\mathcal{F}^{\mathrm{I}}[\mathrm{m}]+\mathcal{F}^{\mathrm{G}}[\mathrm{m}] \\
\mathcal{F}^{\mathrm{L}}[\mathrm{m}] & =\int \mathrm{d} \mathbf{x}\left(-\frac{\varpi}{2} \mathrm{~m}^{2}+\frac{\gamma}{3} \mathrm{~m}^{3}+\frac{\vartheta}{4} \mathrm{~m}^{4}\right) \\
\mathcal{F}^{\mathrm{I}}[\mathrm{m}] & =\int \mathrm{d} \mathbf{x}\left(\frac{\epsilon}{2}[\nabla \mathrm{m}]^{2}\right) \\
\mathcal{F}^{\mathrm{G}}[\mathrm{m}] & =\frac{\alpha}{2} \int \mathrm{d} \mathbf{x}\left(\int \mathrm{d} \mathbf{x}^{\prime} \mathrm{G}\left(\mathbf{x}-\mathbf{x}^{\prime}\right) \mathrm{m}\left(\mathbf{x}^{\prime}\right)\right) \mathrm{m}(\mathbf{x})
\end{aligned}
$$

The chemical potential can then be computed as:

$$
\begin{aligned}
\mu(\mathbf{x}) & =\frac{\delta \mathcal{F}}{\delta \mathrm{m}(\mathbf{x})}=\mu^{\mathrm{L}}(\mathbf{x})+\mu^{\mathrm{I}}(\mathbf{x})+\mu^{\mathrm{G}}(\mathbf{x}) \\
\mu^{\mathrm{L}}(\mathbf{x}) & =-\varpi \mathrm{m}+\gamma \mathrm{m}^{2}+\vartheta \mathrm{m}^{3} \\
\mu^{\mathrm{I}}(\mathbf{x}) & =-\epsilon \triangle \mathrm{m} \\
\mu^{\mathrm{G}}(\mathbf{x}) & =\alpha \int \mathrm{d} \mathbf{x}^{\prime} \mathrm{G}\left(\mathbf{x}-\mathbf{x}^{\prime}\right) \mathrm{m}\left(\mathbf{x}^{\prime}\right)
\end{aligned}
$$

And the Hessian coefficients as:

$$
\begin{aligned}
H\left(\mathbf{x}, \mathbf{x}^{\prime}\right) & =\frac{\delta^{2} \mathcal{F}}{\delta m\left(\mathbf{x}^{\prime}\right) \delta \mathrm{m}(\mathbf{x})}=\mathrm{H}^{\mathrm{L}}+\mathrm{H}^{\mathrm{I}}+\mathrm{H}^{\mathrm{G}} \\
\mathrm{H}^{\mathrm{I}} & =\left(-\boldsymbol{\Phi}+2 \gamma \mathrm{m}+3 \vartheta \mathrm{m}^{2}\right) \delta\left(\mathbf{x}-\mathbf{x}^{\prime}\right) \\
\mathrm{H}^{\mathrm{L}} & =-\epsilon \triangle\left[\delta\left(\mathbf{x}-\mathbf{x}^{\prime}\right)\right] \\
\mathrm{H}^{\mathrm{G}} & =\alpha \mathrm{G}\left(\mathbf{x}-\mathbf{x}^{\prime}\right)
\end{aligned}
$$

Details of the mathematical treatment of the terms describing the formation of the interface and the long-range contribution in the previous expressions are hereby provided. In particular, the numerical implementation of equation 2.46 is shown in detail on page 34 . 
For the chemical potential, the derivation of $\mu^{\mathrm{I}}$ from $\mathcal{F}^{\mathrm{I}}$ is further described to include numerical details related to the collocation lattice used for computation (see equations 2.62 to 2.65). In the case of the long-range contribution in the chemical potential, the special case of the functional derivative of a function is required to obtain equation 2.44 from equation 2.41 :

$$
\frac{\delta m\left(\mathbf{x}^{\prime}\right)}{\delta m(\mathbf{x})}=\delta\left(\mathbf{x}-\mathbf{x}^{\prime}\right)
$$

and by using the product rule:

$$
\begin{aligned}
\mu^{G}(\mathbf{x})= & \frac{\delta \mathcal{F}^{G}[m]}{\delta m(\mathbf{x})} \\
= & \frac{\delta}{\delta m(\mathbf{x})}\left[\frac{\alpha}{2} \int \mathrm{d} \mathbf{x}^{\prime} \int \mathrm{d} \mathbf{x}^{\prime \prime} \mathrm{m}\left(\mathbf{x}^{\prime}\right) \mathrm{G}\left(\mathbf{x}^{\prime}-\mathbf{x}^{\prime \prime}\right) \mathrm{m}\left(\mathbf{x}^{\prime \prime}\right)\right] \\
= & \frac{\alpha}{2} \int \mathrm{d} \mathbf{x}^{\prime} \int \mathrm{d} \mathbf{x}^{\prime \prime}\left[\frac{\delta \mathrm{m}\left(\mathbf{x}^{\prime}\right)}{\delta \mathrm{m}(\mathbf{x})} \mathrm{G}\left(\mathbf{x}^{\prime}-\mathbf{x}^{\prime \prime}\right) \mathrm{m}\left(\mathbf{x}^{\prime \prime}\right)\right. \\
& \left.\quad+\mathrm{m}\left(\mathbf{x}^{\prime}\right) \mathrm{G}\left(\mathbf{x}^{\prime}-\mathbf{x}^{\prime \prime}\right) \frac{\delta \mathrm{m}\left(\mathbf{x}^{\prime \prime}\right)}{\delta \mathrm{m}(\mathbf{x})}\right] \\
= & \frac{\alpha}{2} \int \mathrm{d} \mathbf{x}^{\prime \prime} \mathrm{G}\left(\mathbf{x}-\mathbf{x}^{\prime \prime}\right) \mathrm{m}\left(\mathbf{x}^{\prime \prime}\right)+\frac{\alpha}{2} \int \mathrm{d} \mathbf{x}^{\prime} \mathrm{G}\left(\mathbf{x}^{\prime}-\mathbf{x}\right) \mathrm{m}\left(\mathbf{x}^{\prime}\right) \\
= & \alpha \int \mathrm{d} \mathbf{x}^{\prime} \mathrm{G}\left(\mathbf{x}^{\prime}-\mathbf{x}\right) \mathrm{m}\left(\mathbf{x}^{\prime}\right)
\end{aligned}
$$

To obtain expressions for the Hessian coefficients, equation 2.46 results from applying equation 2.48 to equation 2.43. In the case of the long-range term, equation 2.47 is obtained by the definition of the functional derivative applied to equation 2.44 .

For the computational study, the free energy, the chemical potential and the Hessian matrix are computed in a collocation lattice, therefore equations 2.39 to 2.47 in discrete space are needed. In the later, the free energy functional becomes then a multivariate function of $m_{i}$ with $i=0 \ldots N$, where $N$ is the number of grid cells in the collocation lattice and $\boldsymbol{\Delta}$ is the size of a grid cell which in the case of a $2 \mathrm{D}$ lattice is $\boldsymbol{\Delta}=\Delta_{x} * \Delta_{y}$. The order parameter $\mathrm{m}$ can then be treated as a vector of $\mathrm{N}$ scalar components during the calculations, even for three dimensional systems where the collocation grid can be transformed into a vector to use linear storage during the implementation in C or other programming language intended for high performance computing.

Therefore, the numerical approximation of the Ohta-Kawasaki free energy functional in equations 2.39 to 2.41 is shown below: 


$$
\begin{aligned}
& \mathcal{F}[\mathrm{m}(\mathbf{x})] \simeq \mathcal{F}(\{\mathfrak{m}\})=\mathcal{F}^{\mathrm{L}}(\{\mathrm{m}\})+\mathcal{F}^{\mathrm{I}}(\{\mathrm{m}\})+\mathcal{F}^{\mathrm{G}}(\{\mathrm{m}\}) \\
& \mathcal{F}^{\mathrm{L}}(\{\mathrm{m}\})=\sum_{i=1}^{\mathrm{N}} \boldsymbol{\Delta}\left(-\frac{\varpi}{2} \mathrm{~m}_{\mathrm{i}}^{2}+\frac{\gamma}{3} \mathrm{~m}_{\mathrm{i}}^{3}+\frac{\vartheta}{4} \mathrm{~m}_{\mathrm{i}}^{4}\right) \\
& \left.\mathcal{F}^{\mathrm{I}}(\{\mathrm{m}\})=\sum_{i=1}^{N} \boldsymbol{\Delta}\left(\frac{\epsilon}{2}[\nabla\{\mathfrak{m}\}\}_{i}\right]^{2}\right) \\
& \mathcal{F}^{\mathrm{G}}(\{m\})=\frac{\alpha}{2} \sum_{i=1}^{N} \Delta m_{i}\left(\sum_{j=1}^{N} \Delta \mathrm{G}(i, j) m_{j}\right)
\end{aligned}
$$

For the chemical potential, the transformation of the functional derivative in continuum space to partial derivatives in discrete space is considered:

$$
\begin{aligned}
\mu(\mathbf{x}) & =\frac{\delta \mathcal{F}}{\delta m(\mathbf{x})} \simeq \frac{1}{\Delta} \frac{\partial \mathcal{F}}{\partial m_{i}}=\mu_{i} \\
\mu_{i}^{\mathrm{L}} & \simeq-\varpi m_{i}+\gamma m_{i}^{2}+\vartheta m_{i}^{3} \\
\mu_{i}^{I} & \simeq-\left.\epsilon(\triangle m)\right|_{i} \\
\mu_{i}^{G} & \simeq \alpha \sum_{j=1}^{N} \Delta G(i, j) m_{j}
\end{aligned}
$$

The transformation from equation 2.54 to equation 2.58 is shown in detail below using a 2D lattice where the position $i$ in the lattice is defined by the Cartesian coordinates $(k, l)$. Using the simple backward finite-differences scheme for the numerical approximations of the definitions in continuum space, the gradient vector of the order parameter and the square gradient term in equation 2.54 can be expressed as:

$$
\begin{aligned}
\nabla m(\mathbf{x}) & =\left(\frac{\partial m}{\partial x}, \frac{\partial m}{\partial y}\right) \\
& \left.\simeq\left(\frac{m_{k, l}-m_{k-1, l}}{\triangle_{x}}, \frac{m_{k, l}-m_{k, l-1}}{\triangle_{y}}\right) \simeq(\nabla m)\right|_{i} \\
(\nabla m(\mathbf{x}))^{2} & =\nabla m(\mathbf{x}) \cdot \nabla m(\mathbf{x}) \\
& =\left(\frac{\partial m}{\partial x}\right)^{2}+\left(\frac{\partial m}{\partial y}\right)^{2} \\
& \simeq\left(\frac{\left(m_{k, l}-m_{k-1, l}\right)^{2}}{\triangle_{x}^{2}}+\frac{\left(m_{k, l}-m_{k, l-1}\right)^{2}}{\triangle_{y}^{2}}\right) \simeq\left[\left.(\nabla m)\right|_{i}\right]^{2}
\end{aligned}
$$


The first derivative of equation 2.54 is then:

$$
\begin{aligned}
& \frac{\partial \mathcal{F}^{\mathrm{I}}}{\partial \mathrm{m}_{\mathrm{i}}} \simeq \frac{\boldsymbol{\Delta} \epsilon}{2} \frac{\partial}{\partial \mathrm{m}_{\mathrm{i}}}\left[\sum_{\mathrm{k}, \mathrm{l}}\left[(\nabla \mathrm{m})_{\mathrm{i}}\right]^{2}\right] \\
& \simeq \frac{\boldsymbol{\Delta} \epsilon}{2}\left[\frac{2}{\triangle_{x}^{2}}\left(m_{k, l}-m_{k-1, l}\right)-\frac{2}{\triangle_{x}^{2}}\left(m_{k+1, l}-m_{k, l}\right)+\cdots\right] \\
& \simeq \frac{\Delta \epsilon}{2}\left[\frac{2}{\triangle_{x}^{2}}\left(2 m_{k, l}-m_{k-1, l}-m_{k+1, l}\right)+\frac{2}{\triangle_{y}^{2}}\left(2 m_{k, l}-m_{k, l+1}-m_{k, l+1}\right)\right] \\
& \simeq-\Delta \epsilon\left[\frac{1}{\triangle_{x}^{2}}\left(m_{k-1, l}+m_{k+1, l}-2 m_{k, l}\right)+\frac{1}{\triangle_{y}^{2}}\left(m_{k, l-1}+m_{k, l+1}-2 m_{k, l}\right)\right] \\
& \simeq-\Delta \epsilon\left[\left.\frac{\partial^{2} \mathrm{~m}}{\partial x^{2}}\right|_{(k, l)}+\left.\frac{\partial^{2} \mathrm{~m}}{\partial y^{2}}\right|_{(k, l)}\right]
\end{aligned}
$$

The expression in square brackets in equation 2.63 is the central difference approximation of the Laplace operator. Considering this and equation 2.56:

$$
\begin{aligned}
\frac{\partial \mathcal{F}^{\mathrm{I}}}{\partial \mathrm{m}_{\mathrm{i}}} & =-\mathbf{\Delta} \epsilon(\Delta \mathrm{m})_{(\mathrm{k}, \mathrm{l})} \\
\frac{\delta \mathcal{F}^{\mathrm{I}}}{\delta \mathrm{m}(\mathbf{x})} & \simeq \frac{1}{\boldsymbol{\Delta}} \frac{\partial \mathcal{F}^{\mathrm{I}}}{\partial \mathrm{m}_{\mathrm{i}}}=-\epsilon(\Delta \mathrm{m})_{(\mathrm{k}, \mathrm{l})}
\end{aligned}
$$

Equation 2.65 is the 2D case of equation 2.58. For the implementation of the Laplace operator in equation 2.65, an extended stencil is used to improve the accuracy of the numerical approximation, as shown later on page 34 .

For the Hessian coefficients, the functional derivative of $\mu(\mathbf{x})$ is computed:

$$
\begin{aligned}
H\left(\mathbf{x}, \mathbf{x}^{\prime}\right) & =\frac{\delta^{2} \mathcal{F}}{\delta m\left(\mathbf{x}^{\prime}\right) \delta m(\mathbf{x})}=\frac{\delta}{\delta m\left(\mathbf{x}^{\prime}\right)}\left[\frac{\delta \mathcal{F}}{\delta m(\mathbf{x})}\right] \\
& =\frac{\delta \mu(\mathbf{x})}{\delta m\left(\mathbf{x}^{\prime}\right)}
\end{aligned}
$$

and the relation between the Dirac delta and the Kronecker delta:

$$
\delta\left(\mathbf{x}-\mathbf{x}^{\prime}\right) \simeq \frac{\delta_{i, j}}{\boldsymbol{\Delta}}
$$

are needed to proceed with the numerical calculations. 
The functional derivative of equations 2.57 to 2.59 provides the expressions for the numerical approximation of the Hessian coefficients:

$$
\begin{aligned}
& \mathrm{H}_{i, j}^{\mathrm{L}} \simeq\left(-\varpi+2 \gamma \mathrm{m}_{i}+3 \vartheta \mathrm{m}_{i}^{2}\right) \frac{\delta_{i, j}}{\boldsymbol{\Delta}} \\
& \mathrm{H}_{i, j}^{\mathrm{I}} \simeq(-\epsilon \triangle) \frac{\delta_{i, j}}{\boldsymbol{\Delta}} \\
& \mathrm{H}_{i, j}^{\mathrm{G}} \simeq \alpha \mathrm{G}(i, j)
\end{aligned}
$$

The first term contributes only to values of the diagonal of the Hessian matrix (i.e. $i=j$ ). The second term, $H_{i, j}^{I}$, also modifies values of the diagonal. However other values of the Hessian matrix are also modified by the calculation of this term due to the additional cells needed for the numerical computation of Laplace operator in equation 2.69. The third term, $\mathrm{H}_{i, j}^{\mathrm{G}}$, contributes to all the entries in the Hessian matrix. This last term accounting for the long range interactions described by the Greens function, explicitly indicates the Hessian matrix is dense. Also, the Hessian matrix is symmetric due to $H_{i, j} \equiv H_{j, i}$.

For the computational studies, the free energy of the system is computed using equations 2.53 to 2.55 , the chemical potential is computed using equations 2.57 to 2.59 and the Hessian coefficients using equations 2.68 to 2.70 . Complete expressions for $\mathcal{F}(\{m\}), \mu_{i}$ and $H_{i, j}$ are shown below:

$$
\begin{aligned}
\mathcal{F}(\{m\})= & \Delta \sum_{i=1}^{N}\left(-\frac{\varpi}{2} m_{i}^{2}+\frac{\gamma}{3} m_{i}^{3}+\frac{\vartheta}{4} m_{i}^{4}+\frac{\epsilon}{2}\left[\nabla m_{i}\right]^{2}\right) \\
& +\frac{\alpha}{2} \Delta^{2} \sum_{i=1}^{N} m_{i}\left(\sum_{j=1}^{N} G(i, j) m_{j}\right) \\
\mu_{i}= & -\varpi m_{i}+\gamma m_{i}^{2}+\vartheta m_{i}^{3}-\epsilon(\Delta m)_{i}+\alpha \sum_{j=1}^{N} \Delta G(i, j) m_{j} \\
H_{i, j}= & \frac{1}{\Delta}\left(-\varpi+2 \gamma m_{i}+3 \vartheta m_{i}^{2}\right) \delta_{i, j}-\frac{\epsilon}{\Delta} \Delta \delta_{i, j}+\alpha G(i, j)
\end{aligned}
$$

Different numerical schemes to compute the Laplace operator $\triangle$ in equations 2.72 and 2.73 are available, where the central finite-differences is one of the simplest and most commonly used. In that later scheme, the computation of the operator is anisotropic: only grid points along the direction of the axes are included. Due to this directional bias, the Laplace operator is not completely rotational invariant and a discretization error or grid-related artifact is introduced 
by the use of an anisotropic scheme [76-78]. Snapshots of grid-related artifacts during the phase separation process in binary blends using the Cahn-HilliardCook equation are shown by Sevink [79], who also highlights the importance of avoiding this rotational bias to obtain proper structure factors in the study of block copolymer systems using field-methods like the Ohta-Kawasaky.

Despite providing a greater rotational symmetry for the Laplace operator, Isotropic stencils involve more grid points in the calculation therefore increasing the computational cost. Patra and Karttunen [77] show that the extra cost of using an isotropic stencil is usually less than $20 \%$, for matrices with $10^{6}$ to $10^{8}$ elements in single-core processors using the maximum optimization level available by the compiler. The overall extra cost of computing the Laplace operator with an isotropic stencil can be diminish by the use of parallel computing, specially for large matrices.

Therefore, I decide to use an isotropic stencil in the computational studies in order to avoid grid-related artifacts in the simulation results and further conclusions. The calculation of the terms in equations 2.72 and 2.73 requiring the Laplace operator is described below for the 2D case using an isotropic 9-points stencil (Fig. 2.6) also known as Mehrstellen or D2Q9 stencil.

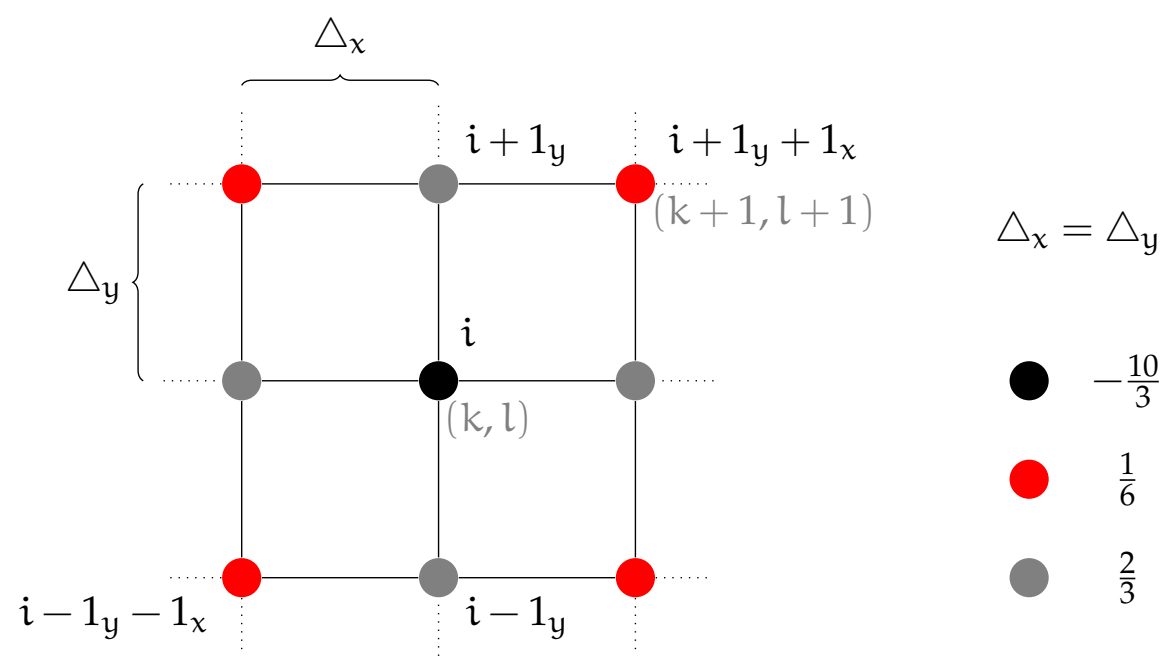

Figure 2.6: Square 9-points stencil for the numerical approximation of the Laplace operator. Coefficients for each grid cell included in stencil are shown. The cell $i=(k, l)$ where the operator is computed is depicted in black.

In a 2D collocation lattice, a grid cell $i$ can be identified in terms of its Cartesian coordinates $(x, y)$ as $i=(k, l)$ to easily locate the neighboring cells considered by the numerical stencil of the Laplace operator. Nearest neighbors along 
every axis are then the cells $i+1_{x}=(k+1, l), i-1_{x}=(k-1, l), i+1_{y}=(k, l+1)$ and $i-1_{y}=(k, l-1)$. The numerical computation of the term accounting for the cost of an interface in the chemical potential (cf. equation 2.72) is now straightforward by applying the stencil shown in figure 2.6 to the order parameter field in the grid cell $i$. However, the calculation of the corresponding Hessian coefficients $H_{i, j}^{I}$ might require a more detailed description.

Recalling that for the chosen stencil $\triangle_{x}=\triangle_{y}$ and $\boldsymbol{\Delta}=\triangle_{x}^{2}, H_{i, j}^{I}$ is then:

$$
\begin{aligned}
H_{i, j}^{I} & =-\frac{\epsilon}{\boldsymbol{\Delta}}\left[\frac{1}{\triangle_{x}^{2}}\left\{-\frac{10}{3} \delta_{i, j}+\frac{2}{3}\left(\delta_{i \pm 1_{x}, j}+\delta_{i \pm 1_{y}, j}\right)+\frac{1}{6}\left(\delta_{i \pm 1_{x}+1_{y}, j}+\delta_{i \pm 1_{x}-1_{y}, j}\right)\right\}\right] \\
& =\epsilon_{1} \delta_{i, j}+\epsilon_{2}\left(\delta_{i \pm 1_{x, j}}+\delta_{i \pm 1_{y}, j}\right)+\epsilon_{3}\left(\delta_{i \pm 1_{x}+1_{y, j}}+\delta_{i \pm 1_{x}-1_{y}, j}\right)
\end{aligned}
$$

with:

$$
\epsilon_{1}:=\frac{10}{3} \frac{\epsilon}{\boldsymbol{\Delta}^{2}} \quad \epsilon_{2}:=-\frac{2}{3} \frac{\epsilon}{\boldsymbol{\Delta}^{2}} \quad \epsilon_{3}:=-\frac{1}{6} \frac{\epsilon}{\boldsymbol{\Delta}^{2}}
$$

An example of the contribution to the Hessian matrix due to the computation of $\mathrm{H}_{i, j}^{\mathrm{I}}$ for an order parameter field $\{\mathrm{m}\}$ discretized in a collocation lattice of size $(4,3)$ is shown in figure 2.7 .

\section{Implementation of the Greens function}

The Greens function representing the long range interactions in equation 2.41, obeys the Poisson's equation:

$$
\triangle \mathrm{G}\left(\mathbf{x}-\mathbf{x}^{\prime}\right)=-\delta\left(\mathbf{x}-\mathbf{x}^{\prime}\right)+\hat{\mathbf{c}}
$$

Where the constant $\hat{c}$ represents the compensating charge uniformly distributed, using the analogy from electrostatics.

Solutions are known in the case of large system sizes where the effect of boundary conditions can be negligible $[49,80]$ :

$$
\begin{array}{ll}
G\left(\mathbf{x}-\mathbf{x}^{\prime}\right)=-\frac{\left|\mathbf{x}-\mathbf{x}^{\prime}\right|}{2} & \text { in 1D } \\
G\left(\mathbf{x}-\mathbf{x}^{\prime}\right)=-\frac{1}{2 \pi} \ln \left(\left|\mathbf{x}-\mathbf{x}^{\prime}\right|\right) & \text { in 2D } \\
G\left(\mathbf{x}-\mathbf{x}^{\prime}\right)=\frac{1}{4 \pi} \frac{1}{\left|\mathbf{x}-\mathbf{x}^{\prime}\right|} & \text { in 3D }
\end{array}
$$

However, finite-size systems occur in computer simulations and the choice of appropriate boundary conditions for the system investigated are required 


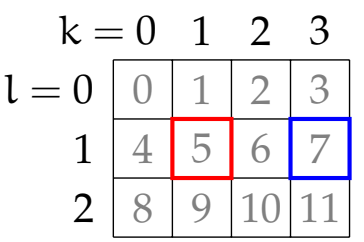

Collocation grid for $\{m\}$

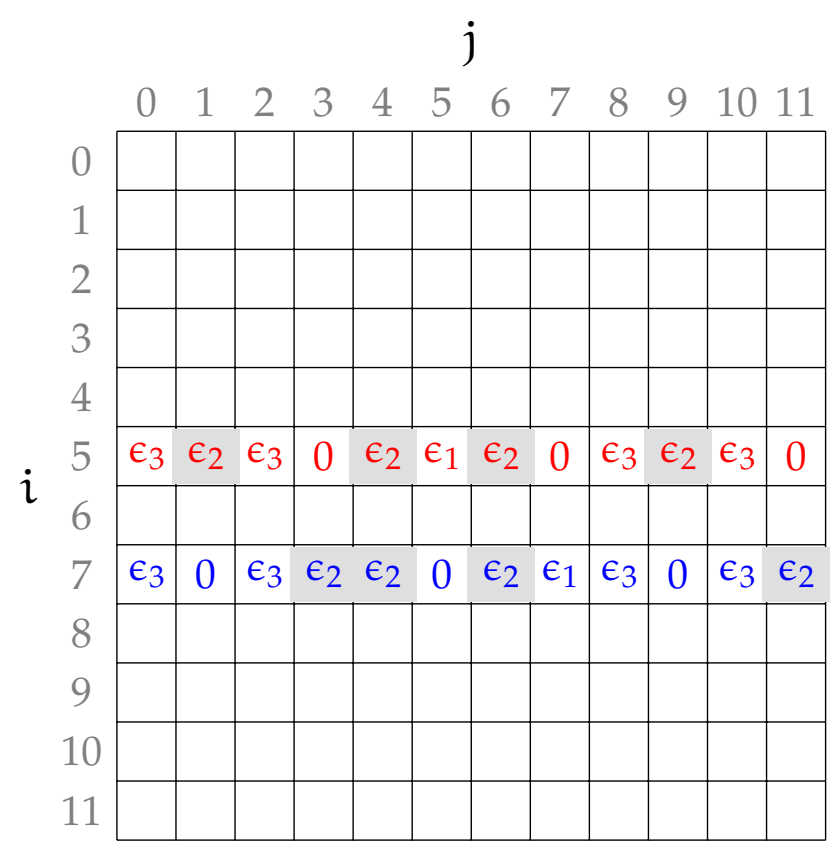

$H_{i, j}^{I}$

Figure 2.7: Example of two $H_{i, j}^{I}$ entries for the corresponding cells in the $\{m\}$ grid. Red values in the Hessian matrix correspond to $\mathrm{H}_{5, j}^{\mathrm{I}}$, blue correspond to $\mathrm{H}_{7, j}^{\mathrm{I}}$ and entries highlighted in gray correspond to nearest neighbors considering the boundary conditions.

and must be considered to find $G\left(\mathbf{x}-\mathbf{x}^{\prime}\right)$. For a finite-size system in a regular grid with periodic boundary conditions, the constant $\hat{c}$ in equation 2.75 is:

$$
\begin{array}{ll}
\hat{c}=\frac{1}{L} & \text { in } 1 D \\
\hat{c}=\frac{1}{A}=\frac{1}{L_{X} L_{Y}} & \text { in 2D } \\
\hat{c}=\frac{1}{V}=\frac{1}{L_{X} L_{Y} L_{Z}} & \text { in 3D }
\end{array}
$$

Finding the Greens function for confined systems or for boundary conditions other than periodic is a challenging task that has been previously studied in our group [81], mainly because the Ohta-Kawasaky model was developed for bulk systems and the assumption of a constant monomer density used for the fitting of the inverse structure factor is no longer valid in a confined system. 
Therefore, the choice of appropriate boundary conditions mimicking the interaction of the diblock copolymer system with hard surfaces (i.e. the change in monomer density in the vicinity of a hard wall and no flux through the confining surface) is still an open challenge. According to Silverberg's argument [82], in the vicinity of a hard wall chain conformations can be described as random walks reflected at the surface. Therefore, the use of reflective boundary conditions for computer simulations of confined systems is valid. However, in the case of thin films of very small thickness, the structure of the segments of a single chain becomes compact changing the monomer density [83]. In this later case, additional considerations are needed when using the Ohta-Kawasaki model.

The Fourier transform of the Greens function and the delta function is used to calculate equation 2.75 for the case of periodic boundary conditions. The central difference stencil for the Laplace operator allows an easier calculation of the left-hand side of the equation, when compared to the extended stencil used for more accurate and stable calculations. Since the long-range term in the Ohta Kawasaki free energy functional is a convolution of the order parameter with the Greens function, this calculation is not the most prone to numerical discretization errors, therefore the use of a different stencil to ease the calculation is a reasonable choice.

The calculation mentioned above is now described in detail for a two dimensional system of size $A=L_{x} \cdot L_{y}$, discretized on a collocation grid of $N=N_{x} \cdot N_{y}$ cells, each one of them with size $\boldsymbol{\Lambda}=\triangle_{x} * \triangle_{y}$, the Fourier transforms can be expressed in terms of the variables $\mathbf{x}, j$ in real space and $q_{k}, k$ in reciprocal space:

$$
G\left(x-x^{\prime}\right)=\frac{1}{A} \sum_{q_{k}} \tilde{G}\left(q_{k}\right) e^{i q_{k}\left(x-x^{\prime}\right)}
$$

with:

$$
\tilde{G}\left(q_{k}\right)=\int_{A} d(x) G\left(x-x^{\prime}\right) e^{-i q_{k}\left(x-x^{\prime}\right)}
$$

where:

$$
\begin{aligned}
\mathbf{x}_{j} & =\left(\Delta_{x} \cdot j_{x}, \Delta_{y} \cdot j_{y}\right) & 0 & \leqslant j_{x, y}<N_{x, y} \\
q_{k} & =\left(\frac{2 \pi}{L_{x}} k_{x}, \frac{2 \pi}{L_{y}} k_{y}\right) & 0 & \leqslant k_{x, y}<N_{x, y}
\end{aligned}
$$

Using the central difference stencil to calculate the Laplace operator and the equation 2.82 to compute the Greens function, the left-hand side of equation 2.75 
can then be expressed as:

$$
\begin{aligned}
& \triangle \mathrm{G}\left(\mathbf{x}-\mathbf{x}^{\prime}\right) \simeq \frac{1}{\triangle_{x}^{2}}\left(\mathrm{G}\left(\mathbf{x}-\mathbf{x}^{\prime}+\triangle_{\mathbf{x}}\right)+\mathrm{G}\left(\mathbf{x}-\mathbf{x}^{\prime}-\triangle_{\mathbf{x}}\right)-2 \mathrm{G}\left(\mathbf{x}-\mathbf{x}^{\prime}\right)\right)+ \\
& \frac{1}{\triangle_{y}^{2}}\left(\mathrm{G}\left(\mathbf{x}-\mathbf{x}^{\prime}+\triangle_{\mathbf{y}}\right)+\mathrm{G}\left(\mathbf{x}-\mathbf{x}^{\prime}-\triangle_{\mathbf{y}}\right)-2 \mathrm{G}\left(\mathbf{x}-\mathbf{x}^{\prime}\right)\right) \\
&= \frac{1}{A} \sum_{q_{k}} \tilde{G}\left(\mathrm{q}_{k}\right) e^{i q_{k}\left(\mathbf{x}-\mathbf{x}^{\prime}\right)}\left\{\frac{1}{\triangle_{x}^{2}}\left[e^{i q_{k} \triangle_{x}}+e^{-i q_{k} \triangle_{x}}-2\right]+\right. \\
&\left.\frac{1}{\triangle_{y}^{2}}\left[e^{i q_{k} \triangle_{y}}+e^{-i q_{k} \triangle_{y}}-2\right]\right\} \\
&=\frac{1}{A} \sum_{q_{k}} \tilde{G}\left(q_{k}\right) e^{i q_{k}\left(x-x^{\prime}\right)}\left\{\frac{2}{\triangle_{x}^{2}}\left[\cos \left(q_{k} \triangle_{x}\right)-1\right]+\right. \\
&=-\frac{1}{A} \sum_{q_{k} \neq 0} \tilde{G}\left(q_{k}\right) e^{i q_{k}\left(x-x^{\prime}\right)} \tilde{Q}\left(q_{k}\right)
\end{aligned}
$$

with:

$$
\begin{aligned}
\tilde{Q}\left(q_{k}\right) & :=\frac{2}{\triangle_{x}^{2}}\left[1-\cos \left(q_{k} \triangle_{x}\right)\right]+\frac{2}{\triangle_{y}^{2}}\left[1-\cos \left(q_{k} \triangle_{y}\right)\right] \\
& =\frac{2}{\triangle_{x}^{2}}\left[1-\cos \left(2 \pi \frac{k_{x}}{N_{x}}\right)\right]+\frac{2}{\triangle_{y}^{2}}\left[1-\cos \left(2 \pi \frac{k_{y}}{N_{y}}\right)\right]
\end{aligned}
$$

and the right-hand side as:

$$
\begin{aligned}
-\delta\left(\mathbf{x}-\mathbf{x}^{\prime}\right)+\frac{1}{A} & =-\frac{1}{A} \sum_{q_{k}} e^{i q_{k}\left(\mathbf{x}-\mathbf{x}^{\prime}\right)}+\frac{1}{A} \\
& =-\frac{1}{A} \sum_{q_{k} \neq 0} e^{i q_{k}\left(x-x^{\prime}\right)}
\end{aligned}
$$

A solution to equation 2.75 can be found by requiring equations 2.86 and 2.88 to be equal:

$$
\tilde{\mathrm{G}}\left(\mathrm{q}_{\mathrm{k}}\right)=\frac{1}{\tilde{\mathrm{Q}}\left(\mathrm{q}_{\mathrm{k}}\right)} \quad \forall \quad \mathrm{q}_{\mathrm{k}} \neq 0
$$


Therefore:

$$
G\left(x-x^{\prime}\right)=\frac{1}{A} \sum_{q_{k} \neq 0} \frac{e^{i q_{k}\left(x-x^{\prime}\right)}}{\tilde{Q}\left(q_{k}\right)}
$$

Due to the periodicity of $q_{k}$ and the sine function, imaginary terms of the summation cancel out and equation 2.90 can be expressed only in terms of cosine functions:

$$
G\left(x-\mathbf{x}^{\prime}\right)=\frac{1}{A} \sum_{k_{x}=0}^{N_{x}-1} \sum_{k_{y}=0}^{N_{y}-1} \frac{1}{\tilde{Q}\left(q_{k}\right)} \cos \left[2 \pi\left(\frac{k_{x}}{N_{x}}\left(\hat{i}-\hat{i}^{\prime}\right)+\frac{k_{y}}{N_{y}}\left(\hat{j}-\hat{j}^{\prime}\right)\right)\right]
$$

where the case $k_{x}=k_{y}=0$ is not to be computed, and $\hat{i}, \hat{j}$ are integers indicating the index of the cell in the collocation grid in every dimension such that:

$$
0 \leqslant \hat{i}<N_{x} \quad 0 \leqslant \hat{j}<N_{y}
$$

For the special case of $\triangle_{x}=\triangle_{y}$ (i.e. $\triangle_{x}^{2}=\boldsymbol{\Delta}$ ) as in the isotropic stencil for the Laplace operator used for a more accurate calculation of the free energy, the chemical potential and the Hessian coefficients (c.f. page 34), $\tilde{Q}\left(q_{k}\right)$ can be simplified as:

$$
\tilde{Q}\left(q_{k}\right)=\frac{2}{\boldsymbol{\Delta}}\left[2-\cos \left(2 \pi \frac{k_{x}}{N_{x}}\right)-\cos \left(2 \pi \frac{k_{y}}{N_{y}}\right)\right]
$$

and using this latter expression, equation 2.91 can be written as:

$$
G\left(x-x^{\prime}\right)=\frac{1}{2 N} \sum_{k_{x}=0}^{N_{x}-1} \sum_{k_{y}=0}^{N_{y}-1} \frac{\cos \left[2 \pi \frac{k_{x}}{N_{x}}\left(\hat{i}-\hat{i}^{\prime}\right)+2 \pi \frac{k_{y}}{N_{y}}\left(\hat{j}-\hat{j}^{\prime}\right)\right]}{\left[2-\cos \left(2 \pi \frac{k_{x}}{N_{x}}\right)-\cos \left(2 \pi \frac{k_{y}}{N_{y}}\right)\right]}
$$

Expressions for the Greens function in 1D and 3D in periodic boundary conditions can be drawn following the same calculations. For the 1D case, $\mathrm{G}\left(\mathbf{x}-\mathbf{x}^{\prime}\right)$ can be obtained from equation 2.91 by setting $k_{y}=0$ and redefine $\mathrm{A}=\mathrm{L}=\triangle_{\mathrm{x}} \mathrm{N}_{\mathrm{x}}$, and $\mathrm{N}=\mathrm{N}_{\chi}$ :

$$
\begin{aligned}
G\left(x-\mathbf{x}^{\prime}\right)_{1 D} & =\frac{1}{L} \sum_{k_{x}} \frac{1}{\tilde{Q}\left(q_{k}\right)} \cos \left[2 \pi \frac{k_{x}}{N_{x}}\left(\hat{i}-\hat{i^{\prime}}\right)\right] \\
& =\frac{\triangle_{x}}{2 N_{x}} \sum_{k_{x}=1}^{N} \frac{\cos \left[2 \pi \frac{k_{x}}{N_{x}}\left(\hat{i}-\hat{i^{\prime}}\right)\right]}{1-\cos \left(2 \pi \frac{k_{x}}{N_{x}}\right)}
\end{aligned}
$$


The expression for $\mathrm{G}\left(\mathbf{x}-\mathbf{x}^{\prime}\right)$ in 3D can be drawn from equations $2.82,2.87,2.90$ and 2.89:

$$
\begin{aligned}
G\left(x-x^{\prime}\right)_{3 D} & =\frac{1}{V} \sum_{q_{k} \neq 0} \frac{e^{i q_{k}\left(x-x^{\prime}\right)}}{\tilde{Q}\left(q_{k}\right)} \\
& =\frac{1}{V} \sum_{k_{x}=0}^{N_{x}-1} \sum_{k_{y}=0}^{N_{y}-1} \sum_{k_{z}=0}^{N_{z}-1} \frac{\cos \left[2 \pi\left\{\frac{k_{x}}{N_{x}}\left(\hat{i}-\hat{i^{\prime}}\right)+\frac{k_{y}}{N_{y}}\left(\hat{j}-\hat{j}^{\prime}\right)+\frac{k_{z}}{N_{z}}\left(\hat{k}-\hat{k}^{\prime}\right)\right\}\right]}{\tilde{Q}\left(q_{k}\right)}
\end{aligned}
$$

where $\hat{i}, \hat{j}, \hat{k}$ are integers indicating the index of the cell in the collocation grid and:

$$
\tilde{Q}\left(q_{k}\right):=\sum_{\alpha} \frac{2}{\triangle_{\alpha}^{2}}\left[1-\cos \left(2 \pi \frac{k_{\alpha}}{N_{\alpha}}\right)\right] \quad \alpha \in(x, y, z)
$$

Here again the case $k_{x}=k_{y}=k_{z}=0$ is not to be computed.

\subsection{Summary and conclusions}

Continuum models have been employed to optimize parameters for the directed self-assembly (DSA) of block copolymers. By comparing the predictions of two continuum free-energy functionals - the Swift-Hohenberg model and the Ohta-Kawasaki model - to the predictions of self-consistent field theory (SCFT), we have evaluated their abilities to predict DSA-relevant properties such as the excess free energy of metastable dislocation pairs, the shape of the internal $A B$ interfaces in the vicinity of a defect, and the kinetics of defect motion.

The Swift-Hohenberg model predicts a spurious phase coexistence between a spatially modulated structure and a homogeneous structure that differs in composition.[41,48] Additionally, we show that it only provides a rather poor prediction of the geometry of a prototypical, metastable defect.

The Ohta-Kawasaki model $[49,55,56]$ avoids the prediction of an unphysical macrophase separation because of the long-range $1 / q^{2}$-term. We demonstrate that the model succeeds in predicting the geometry of defects as well as the transition from metastable dislocation pairs at high incompatibility to unstable ones in the vicinity of the order-disorder transition (ODT).[57] Whereas the quantitative mapping from the model parameter and scales to the molecular parameters within the random-phase approximation (RPA) is only valid in the ultimate vicinity of the order-disorder transition (ODT), we have devised a phenomenological extension by adjusting the length and free-energy scales based on the 
comparison to SCFT. This procedure provides a reasonable agreement for equilibrium properties, including the excess free energy of dislocation-pair defects. Thus the Ohta-Kawasaki model has been proven to be a promising continuum model for studying the directed self-assembly (DSA) of diblock copolymers and design guiding patterns and geometries for device-oriented structures.

For symmetric diblock copolymers, the third-order term $\tilde{\gamma}$ vanishes by symmetry, Fig. 2.5 suggests that the RPA-mapping, $\tilde{\alpha}(\chi N)$ in Eq. (2.29), remains rather accurate, and the adjusted free-energy and length scales can be directly read off Figs. 2.2 and 2.3 as a function of $\chi \mathrm{N}$. For asymmetric copolymers, to a first approximation, we expect that $\tilde{\gamma} \sim(2 \mathrm{f}-1)$ with a $\chi \mathrm{N}$-dependent constant of proportionality. In the ultimate vicinity of the ODT, $\tilde{\gamma}$ can be obtained from Leibler's RPA. [24] For intermediate and practically relevant incompatibilities, we suggest to adjust the long-range and third-order coefficients, $\tilde{\alpha}(\chi N, f)$ and $\tilde{\gamma}(\chi N, f)$, as to reproduce (i) the excess free energy of prototypical defects as in Fig. 2.5 and (ii) the location, $\mathrm{f}_{\text {lam } / \text { hex }}$, of the transition between the lamellar and hexagonal phase predicted by SCFT.[...] 


\section{Chapter 3}

\section{Kinetics of self-assembly of block copolymers}

The Ohta-Kawasaki model has been extensively deployed for studying structure formation of block copolymers in the bulk[84]. Despite this model's assumptions and simplifications behind the continuum description of the system, its use provides realistic dynamics for the structure formation process in conjunction with the Cahn-Hilliard dynamics.

Nevertheless, the use of this phenomenological model in the context of directed self-assembly (DSA) poses additional challenges related mainly to the formation and annihilation of defects as part of the ordering process as well as to the interaction with the guiding patterns and confining surfaces. Consequently, this chapter is devoted to the description, both qualitative and quantitative, of DSA in the Ohta-Kawasaki model and to the comparison to selfconsistent field theory and particle-based simulations, illustrating applications to defect motion and annihilation and the design of guiding patterns for fabricating device-oriented structures. In addition, we discuss the significance of the disparity between the characteristic length scale of surface-directed spinodal self-assembly and the equilibrium domain spacing.

The materials presented in this chapter have been published as part of the special collection of papers on the subject, "Advances in Directed Self-Assembly," with the title "Continuum models for directed self-assembly" in the journal Molecular Systems Design \& Engineering. To facilitate the integration of the paper's contents with this dissertation, only the sections of the paper directly related with the scope of this chapter have been included herein, specifically, sections 3.4, 3.5, 3.6 and 4. Part of the content of those sections has been omitted in this chapter and it is indicated by [...], in order to avoid duplication with other chapters in this dissertation.

In integrating the paper's content, the cross-references, citations, and figure 
numbers used in the paper have been adjusted to be consistent with those in this dissertation. The content has been adapted and reproduced from Ref. [28] with permission from the Royal Society of Chemistry. Additional ideas and discussion not included in the published paper are presented in section 3.2.2.

\subsection{Defect motion and annihilation}

In the following we illustrate applications of the Ohta-Kawasaki model to defect structures and non-bulk morphologies, for a symmetric diblock copolymer within the technologically relevant, intermediate segregation regime. Model parameters correspond to $\chi \mathrm{N} \approx 30: \tilde{\alpha}=0.0172$ and $\tilde{\gamma}=0$.

\subsubsection{Dynamics of unstable defects}

The motion of an isolated dislocation core along the stripe direction of a lamellar structure - dislocation climb - does not involve any free-energy barrier by virtue of the translation invariance along the stripes. In contrast, the motion perpendicular to the stripes involves the breaking and reformation of domains. Since our dynamical simulations ignores thermal noise, the concomitant freeenergy barriers $[58,59]$ cannot be overcome and, hence, the thermally activated glide motion does not occur in our Ohta-Kawasaki simulations.

In the following, we study the defect kinetics of apposing dislocations pairs in a lamella structure using the Ohta-Kawasaki model with Cahn-Hilliard dynamics, Eq. (2.32). We focus on the climb motion of a dislocation pair, which has been studied by experiment [85] and simulation.[59] Dislocations with apposing Burgers vector attract each other. Since the glide motion perpendicular to the stripes cannot occur in our model, the distance, $\Delta$, perpendicular to the stripes - the impact parameter - is conserved, whereas the distance, L, along the stripes decreases in response to the strain-field mediated interaction (PeachKoehler forces) between the dislocation cores.

In our study of defect motion we investigate three different values of the impact parameter, $\Delta$, between defect cores: $\Delta / L_{0}=0,1$, and 2 , where $L_{0}=18.28 \xi_{0}$ is the equilibrium domain spacing of the bulk lamellae. The dimension in the direction perpendicular to the stripes is set to $L_{y}=N_{P} L_{0}$ with $N_{P}=8$. Thus, the defect-free lamellar structure is stress-free. The value $N_{p}=8$ is larger than or comparable to the distance between chemical guiding patterns in chemoepitaxy with density multiplication or the distance between topographical features in graphoepithaxy, respectively. Periodic boundary conditions are used in our simulations, mimicking the confining effect of chemical or topographical guiding patterns in DSA with $\mathrm{N}_{\mathrm{p}}$-fold density multiplication; the pinning of the 
internal $A B$ interfaces of the lamellae at the guiding pattern is not considered.

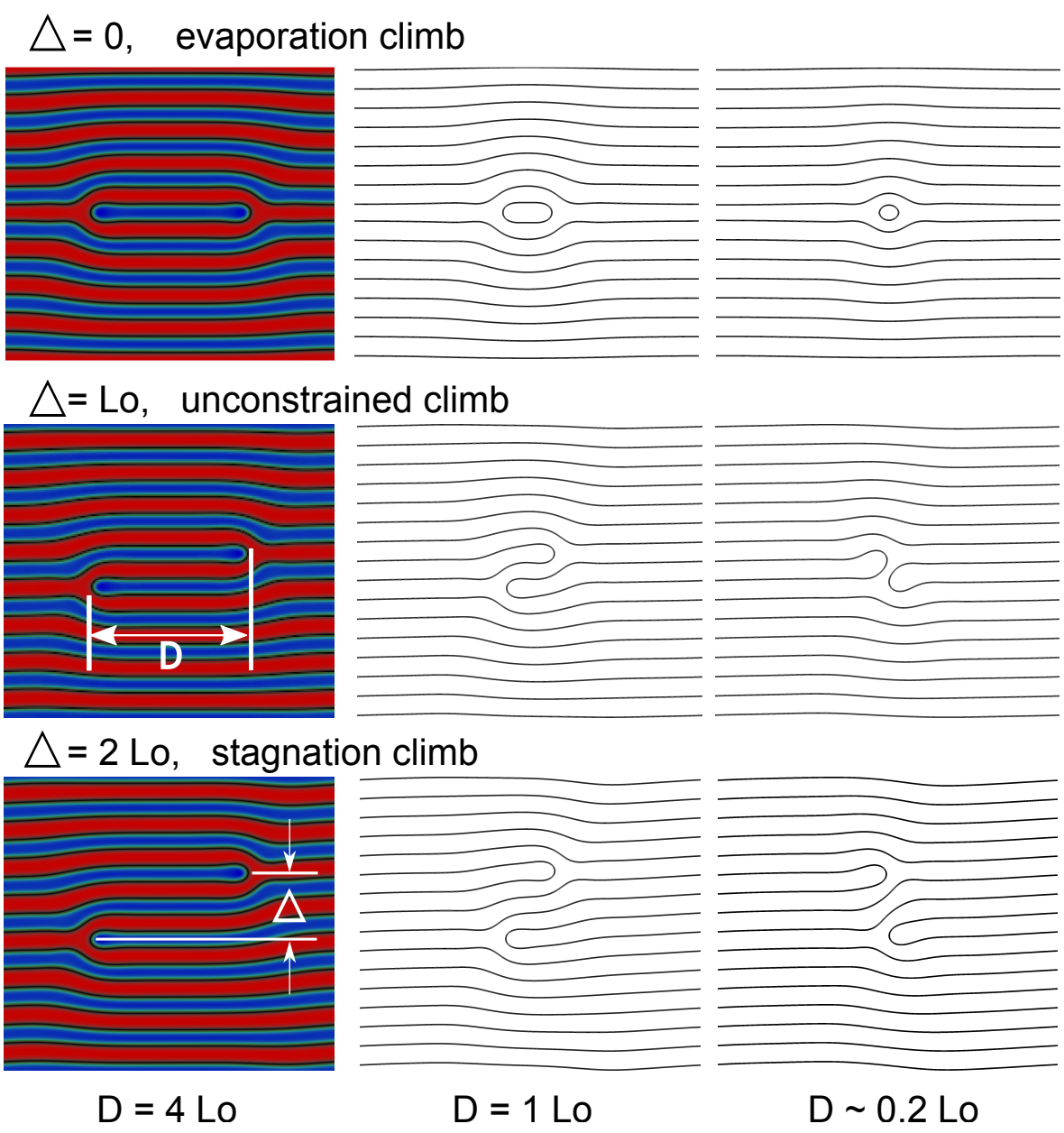

Figure 3.1: Morphology of Ohta-Kawasaki simulations of three dislocation pairs with impact parameter $\Delta / \mathrm{L}_{0}=0,1$, and 2 illustrating the motion of defect cores towards each other. In all cases, the box size is $\mathrm{L}_{x}=8.9 \mathrm{~L}_{0}$ and $\mathrm{L}_{y}=8.0 \mathrm{~L}_{0}$, corresponding to the stress-free lamella in along the $y$-direction. Contour lines (black) show the $A B$ interface at different stages of defect collision.

For all three cases, the initial dislocation pair is unstable, and the defect cores spontaneously approach each other, i.e., D decreases. Fig. 3.1 shows the morphology at different stages during the climb motion. For the cases, $\Delta / \mathrm{L}_{0}=0$ and 1 , dislocations collide and annihilate, forming a defect-free lamellar structure. The morphologies before the annihilation, $\mathrm{D} \approx 0.2 \mathrm{~L}_{0}$, differ: For $\Delta / \mathrm{L}_{0}=0$ the enclosed extra domain continuously shrinks, whereas for $\Delta / \mathrm{L}_{0}=1$, the two domain ends are brought into apposition and join, cf. contour lines for $D \approx 0.2 \mathrm{~L}_{0}$ 
in Fig. 3.1. In the case of $\Delta / L_{0}=2$, a metastable tight dislocation pair is formed, and a final finite value of $\mathrm{D}>0$ remains after the two dislocations pass by each other. We denote this motion as stagnation climb, where the motion stagnates and the system reaches a metastable state, in which the force between the defects vanishes. These observations of the two-dimensional Ohta-Kawasaki model are in agreement with simulations and SCFT calculations. [59]

In order to study the velocity of the defect motion in the direction parallel to the lamellae - dislocation climb - we compute the excess free energy, $\Delta \mathrm{F}_{\mathrm{d}}$, of the defect with respect to the defect-free lamella as a function of $D$. The results are presented in Fig. 3.2.

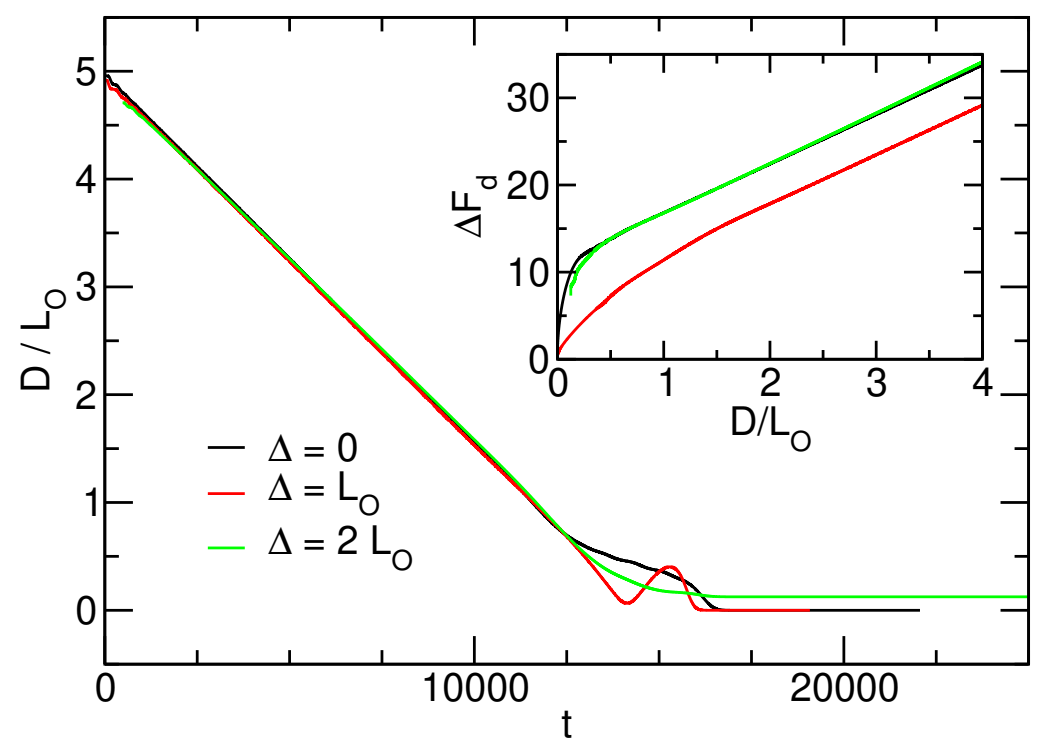

Figure 3.2: Defect motion described by distance, $D$, between defect cores as a function of time $\left(\Lambda=1, \xi_{0}=1\right)$ for impact parameters, $\Delta / L_{0}=0,1$, and 2 . The inset shows the excess free energy, $\Delta F_{d}$, of the defect pair as a function of the distance, D.

Based on the attractive force between defect cores $\mathrm{K}=-\mathrm{d} \Delta \mathrm{F}_{\mathrm{d}} / \mathrm{dD}$, two regimes of defect motion can be identified: an initial stage where the force is largely independent from the distance, D, between cores, and a final stage where the magnitude of $K$ increases as $D$ approaches zero. The force, $K$, is distance-independent for values of $\mathrm{D}>2 \mathrm{~L}_{0}$ in all three cases. For the evaporation and the stagnation climbs, $\Delta / \mathrm{L}_{0}=0$ and 2 , this regime even extends to $\mathrm{D}>\mathrm{L}_{0}$. The distance-independence of the attractive force and the limiting value of $\mathrm{D}>2 \mathrm{~L}_{0}$ for this regime are nicely in agreement with predictions from the minimum free-energy pathway (MFEP) obtained by SCFT calculations[59] for the climb motion of dislocation pairs. 
In the constant-force regime, the velocity of defect motion is virtually independent from the impact parameter $\Delta: \mathrm{dD} / \mathrm{dt}=1 / 2930$ for the evaporation climb, $\mathrm{dD} / \mathrm{dt}=1 / 2900$ for the unconstrained climb and $\mathrm{dD} / \mathrm{dt}=1 / 3000$ in the case of the stagnation climb. This observation differs from particle-based Monte-Carlo simulations,[59] where a factor of 25 is observed between the defect annihilation time for $\Delta=0$ and $\Delta=\mathrm{L}_{0}$, the later being faster. We can rationalize this difference by considering our choice of the Onsager coefficient. According to Eq. (2.32), the Onsager coefficient is independent of the orderparameter. This choice does not captures the barrier in the single-chain dynamics, protracting the evaporation of segments from the encircled domain between the defect cores in the case of $\Delta=0$. Such an intrinsically slow process can be captured by an Onsager coefficient that is proportional to $(1-m)(1+m)$, accounting for the incompressibility of the copolymer melt. Unfortunately, this more appropriate choice of the Onsager coefficient makes the dynamic equation - the equivalent to Eq. (2.32) - non-local posing a computational burden.

\subsubsection{Interaction of dislocations under tension}

The distance-independent force between two apposing dislocation cores differs from the classical Peach-Koehler force, [86] $\mathrm{K} \sim-\frac{1}{\mathrm{D}}$, of two dislocations in the bulk. In previous work,[59] we have rationalized this observation by noting that the lamellae between the apposing dislocation cores - the inner region are compressed due to the presence of an additional stripe, i.e., their lamellar spacing is $\mathrm{L}^{\prime}=\frac{\mathrm{N}_{\mathrm{P}}}{\mathrm{N}_{\mathrm{P}}+1} \mathrm{~L}<\mathrm{L}$, where $\mathrm{L}=\mathrm{L}_{Y} / \mathrm{N}_{\mathrm{P}}=\mathrm{L}_{0}$ is the periodicity of the outer lamellae. Note that the amount of compression depends on the system size, $\mathrm{L}_{Y} \sim \mathrm{N}_{\mathrm{P}}$, perpendicular to the lamellae that, in DSA applications, corresponds to the distance of chemical or topographical lines of the guiding pattern.

Since the outer lamellae adopt their equilibrium spacing whereas the inner lamellae are compressed, the free-energy density of the inner lamellae is higher, and the free energy of the system increases linearly with the length, D, of the inner lamellae. This rational suggests that the force between apposing dislocation defects and thereby the speed, with which they approach each other (and annihilate in the case of $\Delta / L_{0}=0$ and 1), critically depends on the deviation of the lamellar periodicity of the inner and outer lamellae from the equilibrium spacing. In Fig. 3.2 $\mathrm{L}=\mathrm{L}_{0}$ and we maximize the free-energy density difference between the compressed inner and stress-free outer lamellae.

In Fig. 3.3 we investigate the free-energy density, F/A, of defect-free lamellae as a function of the lamellar spacing. The free-energy density exhibits a minimum at $\mathrm{L}=\mathrm{L}_{0}$ and is well described by a quadratic form. The curvature is related to the compression modulus of the lamellae. The crossing of the two 


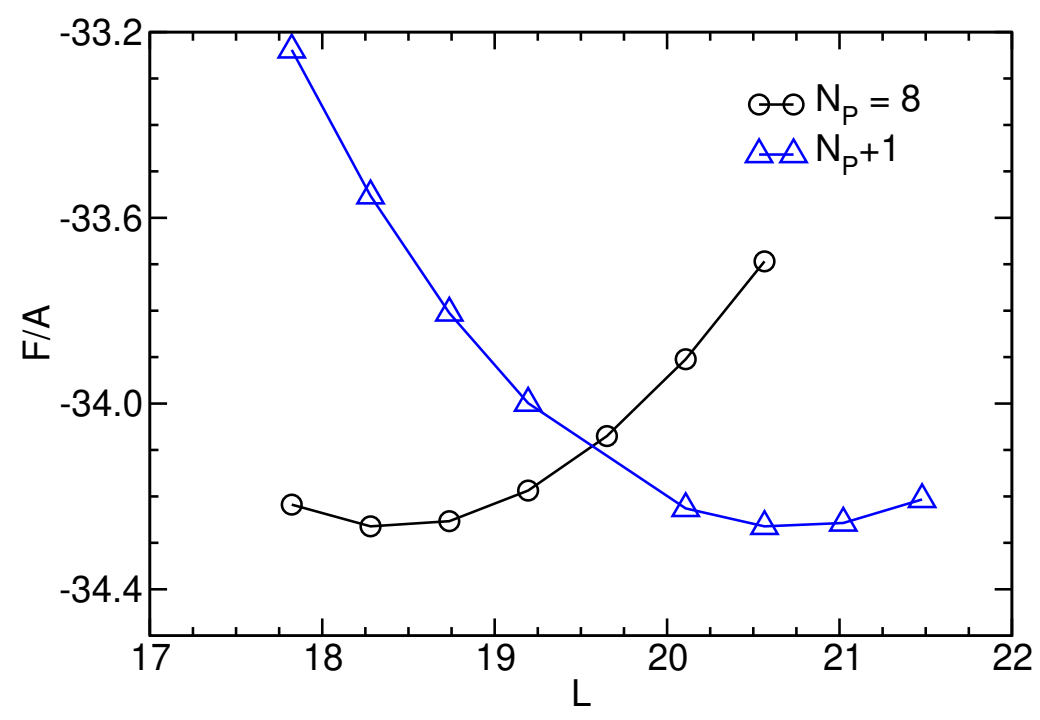

Figure 3.3: Free-energy density F/A as a function of the lamellar spacing $\mathrm{L}=$ $\mathrm{L}_{Y} / 8$ measured in units of the equilibrium periodicity, $\mathrm{L}_{0}$. The free energy is shown for a system comprised of $N_{p}=8$ (black circles) and $8+1$ (blue triangles) lamellae. The crossing point identifies the distance, $\mathrm{L}_{Y}^{*}$. For $\mathrm{L}<\mathrm{L}_{Y}^{*}$ the apposing defect cores with opposite Burgers vectors attract each other.

curves, $F_{N_{p}}(L) / A$ with $N_{p}=8$ and $8+1$, identifies the spacing or distance, $\mathrm{L}_{Y}=\mathrm{N}_{\mathrm{p}} \mathrm{L}$, between the guiding stripes where the free-energy density of the inner, compressed, $N_{p}=8+1$ and outer, expanded lamellae, $N_{p}=8$, is equal and the boundary-induced compression force vanishes. For $N_{p}=8$ this cancellation of the compression force occurs at a mismatch of about $7 \%$. If $L_{y}$ increases further, the outer lamellae are less favorable than the inner ones, and the defects move as to eliminate the outer lamellae of higher free-energy density.

The consequence of a mismatch between the periodicity, $L_{Y}$, of a sparse guiding pattern and the appropriate multiple of the lamellar bulk spacing on the forces between the defect cores is illustrated in Fig. 3.4. We present the excess free-energy, $\Delta F_{d}$, as a function of the distance $D$ between the defect cores for impact parameter, $\Delta=\mathrm{L}_{0}$. At not too small distance, $\mathrm{D}>2 \mathrm{~L}_{0}$, the free energy linearly increases with $D$. The inset of Fig. 3.4 presents the slope, $K=-d \Delta F_{d} / d D$, in this linear regime of $\mathrm{D}$ as a function of the system size, $\mathrm{L}$ in the direction perpendicular to the lamellae. Clearly as the outer lamellae become stretched $\mathrm{L}_{y} / \mathrm{N}_{p}=\mathrm{L}>\mathrm{L}_{0}$, the free-energy difference between the inner and outer lamellae decreases and so does the compression force, $\mathrm{K}$.

The scaling with the perpendicular system size, $L_{Y}=N_{p} L$, can be rationalized as follows: The free-energy density of the lamellae in the vicinity of the 


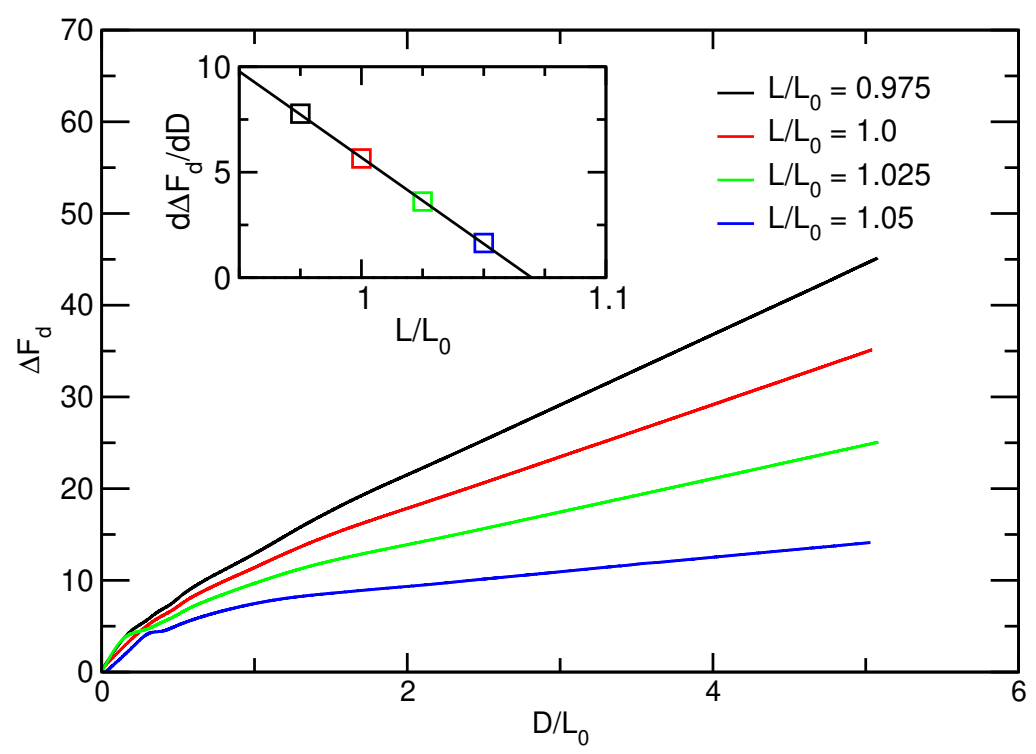

Figure 3.4: Excess free energy $\Delta \mathrm{F}_{\mathrm{d}}$ of defect morphology for different values of lamellar period, $\mathrm{L}$, as a function of the distance, $\mathrm{D}$, between defect cores. The inset presents the slope $d \Delta F_{d} / d D$ as a function of L/L (cf. Eq. (3.2)).

equilibrium spacing $L_{0}$, can be approximated by $F(L) / A \approx f_{0}+\frac{B}{2}\left(\frac{L}{L_{0}}-1\right)^{2}$, where $B$ denotes the compression modulus and $L^{\prime}=L_{Y} /\left(N_{p}+1\right)$. Thus the total free energy of the system can be approximated by

$$
\begin{aligned}
\Delta \mathrm{F}_{\mathrm{d}} & \approx 2 \mathrm{~F}_{\text {core }}+\mathrm{L}_{Y} \mathrm{D} \frac{\mathrm{B}}{2}\left[\left(\frac{\mathrm{L}^{\prime}}{\mathrm{L}_{0}}-1\right)^{2}-\left(\frac{\mathrm{L}}{\mathrm{L}_{0}}-1\right)^{2}\right] \\
& =2 \mathrm{~F}_{\text {core }}+\mathrm{L}_{Y} \mathrm{D} \frac{\mathrm{B}}{2}\left(\frac{\mathrm{L}^{\prime}-\mathrm{L}}{\mathrm{L}_{0}}\right)\left(\frac{\mathrm{L}^{\prime}+\mathrm{L}}{\mathrm{L}_{0}}-2\right) \\
& =2 \mathrm{~F}_{\text {core }}+\frac{\mathrm{B} \mathrm{L}_{Y} \mathrm{D}}{2\left(\mathrm{~N}_{\mathrm{p}}+1\right)^{2}}\left(\frac{\mathrm{L}}{\mathrm{L}_{0}}\right)^{2}\left[1-2\left(\mathrm{~N}_{\mathrm{p}}+1\right)\left(1-\frac{\mathrm{L}_{0}}{\mathrm{~L}}\right)\right]
\end{aligned}
$$

where the reference state is the system with $N_{p}$ lamellae and $F_{\text {core }}$ denotes the excess free energy of the dislocation cores. For the compression force we obtain

$$
\mathrm{K} \approx-\frac{\mathrm{BN}_{\mathrm{p}} \mathrm{L}_{0}}{2\left(\mathrm{~N}_{\mathrm{p}}+1\right)^{2}}\left(\frac{\mathrm{L}}{\mathrm{L}_{0}}\right)^{2}\left[1-\left(2 \mathrm{~N}_{\mathrm{p}}+1\right)\left(\frac{\mathrm{L}}{\mathrm{L}_{0}}-1\right)\right]
$$

This rational suggest that the compression force decreases as the number, $N_{p}$ of lamellae increases, $\mathrm{K} \sim-\frac{1}{\mathrm{~N}_{\mathrm{p}}}$ for $\mathrm{L} \approx \mathrm{L}_{0}$, [59] and that the compression force decreases approximately linearly with $L$ and vanishes if the lamellae are stretched 
to $\frac{\mathrm{L}-\mathrm{L}_{0}}{\mathrm{~L}_{0}} \approx \frac{1}{2 \mathrm{~N}_{\mathrm{p}}+1}$. This prediction is corroborated by the inset of Fig. 3.4, highlighting that the defect motion is very sensitive to a mismatch between the periodicity, $\mathrm{N}_{p} \mathrm{~L}$, of a sparse guiding pattern and the appropriate multiple of the lamellar bulk spacing, $\mathrm{N}_{\mathrm{p}} \mathrm{L}_{0}$.

\subsection{Understanding DSA using a continuum model}

\subsubsection{Stages of directed structure formation}

In section 3.1, we have studied lamellar structures as well as the properties of prototypical defects. A different interesting case for DSA is the design of deviceoriented structures like dots, isolated lines, T-junctions, swirls and others.[5, 7, $14,87]$ Continuum models can be used to investigate the role of guiding patterns for the fabrication of copolymer structures that do not resemble equilibrium bulk morphologies. By virtue of the computational ease, the OhtaKawasaki model can be utilized to systematically explore the geometry and strength of the chemical or topographical guiding patterns in order to obtain the targeted structure and design the guiding pattern to avoid undesired metastable states in the course of DSA. For instance, this strategy has been used for the optimization of grapho-epitaxy-DSA integrated into a conventional lithography process for the case of a contact hole.[88] In the following, we employ twodimensional calculations of the Ohta-Kawasaki model to illustrate the design of geometric confinement and guiding fields to fabricate T-junctions.

Interactions between the guiding pattern and the copolymer material can be straightforwardly incorporated in continuum models by augmenting the freeenergy functional by an additional surface contribution, which describes the wetting behavior [89]

$$
\Delta \mathrm{F}_{\text {wall }}=\int \mathrm{d} \mathbf{x}\left(\mathrm{h}(\mathbf{x}) \mathrm{m}(\mathbf{x})+\frac{1}{2} g(\mathbf{x}) \mathrm{m}^{2}(\mathbf{x})\right)
$$

where the first term, $h$, quantifies the preference of the local substrate as a function of the spatial position, and the second term, $g$, represents the change of the interactions inside the copolymer material in the vicinity of the surface ("missing neighbor effect"). The latter term accounts inter alia for the reduction of the density at the surface that is not explicitly described within the single orderparameter description. In the following, we ignore the latter effect and set $\mathrm{g}=0$.

We start from a perfectly homogeneous state, $m(x) \equiv 0$, at time, $t=0$. Since our calculations ignore thermal fluctuations, the kinetics of structure formation is deterministic. Therefore our calculations highlight how the guiding 
fields direct the self-assembly. Similar to surface-directed spinodal decomposition $[90,91]$ in blends, different stages in the kinetics of structure formation can be distinguished:

1. surface-directed spinodal self-assembly

- linear growth of composition variation driven by surface fields

- exponential amplification of composition fluctuations

2. changes of the domain topology

In the first stage, the surface fields induce the enrichment of the preferred segment species at the location of the guiding field. Including the surface contribution and setting $\mathfrak{m}(\mathbf{x}) \equiv 0$, we obtain from Eq. (2.32)

$$
m(x, t)=\Delta h(x) \cdot \wedge t
$$

for the very initial regime. These guiding-pattern induced composition variations are exponentially amplified by the spinodal kinetics of self-assembly. Linearizing Eq. (2.32), we obtain for the wavevector-dependent growth rate, $r(\mathbf{q})$

$$
r(q) \sim\left(q \xi_{0}\right)^{2}\left[1-\left(q \xi_{0}\right)^{2}\right]+\alpha
$$

Composition variations with the wavevector $q_{\max } \xi_{0}=\frac{1}{\sqrt{2}}$ grow the fastest. Thus, in the course of the surface-directed spinodal self-assembly, a lamellar structure emerges that is registered and aligned with the guiding pattern and is characterized by the periodicity, $\lambda_{\max }$

$$
\lambda_{\max }=2 \sqrt{2} \pi \xi_{0}
$$

Since the linearized form of the SCFT is the RPA, SCFT predicts the growth rate, $r(q) \sim-q^{2} \gamma_{2}(q)$. Fig. 2.1 demonstrates that the Ohta-Kawasaki model, Eq. (2.10), provides a rather accurate approximation for the second-order vertex function. Therefore we expect that the estimate for the periodicity, $\lambda_{\max }$, of the initial lamellar structure also is accurate.

At the order-disorder transition, $\tilde{\alpha}_{c}=\frac{1}{4}$, the periodicity of the spinodal structure coincides with the equilibrium lamellar spacing, $\mathrm{L}_{0}$. Upon increasing the incompatibility, however, we observe that $\lambda_{\max }$ remains unaltered when measured in the natural length unit, $\xi_{0}$, of the Ohta-Kawasaki model (or decreases when measured in units of $R_{e}$ ), whereas the equilibrium lamellar spacing, $\mathrm{L}_{0}$, increases. Using the one-mode approximation that is appropriate for weak segregation, we obtain for the ratio

$$
\frac{\lambda_{\max }}{\mathrm{L}_{0}} \approx \sqrt[4]{4 \tilde{\alpha}}
$$


Also within the SCFT, the ratio $\frac{\lambda_{\max }}{L_{0}}$ decreases as we increase the incompatibility, $\chi \mathrm{N}$. Thus, the lamellar structure that is templated by the surface-directed selfassembly differs from the equilibrium structure. For $\tilde{\alpha}=0.0172$, i.e., $\chi \mathrm{N} \approx 30$ the ratio of length scales is about 0.5 .

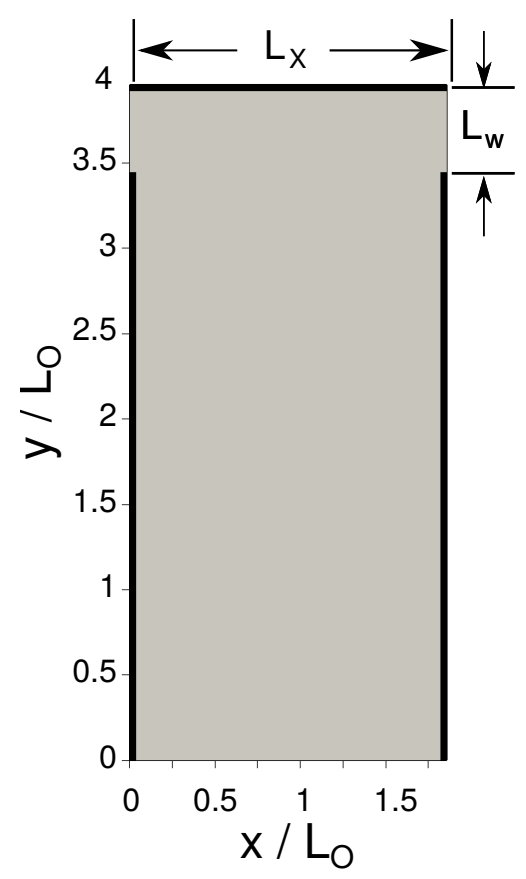

Figure 3.5: Geometric confinement in combination with a guiding pattern (black lines) is used to fabricate device-oriented structures. The width, $\mathrm{L}_{X}$, of the confinement and the geometry, $\mathrm{L}_{W}$, guiding pattern is adjusted to obtain an isolated T-junction.

In order to illustrate the fabrication of T-junctions, we employ a two-dimensional system with reflecting boundary conditions in all directions that mimic confining walls. At the boundaries there acts a guiding field, $h(x, y)$, according to Eq. (3.3) that attracts the A-component with strength -0.3 or that adopts the neutral, non-preferential value 0. The system geometry is sketched in Fig. 3.5. The height of the system is $\mathrm{L}_{Y}=4 \mathrm{~L}_{0}$, the top horizontal boundary attracts the A-component, whereas the bottom horizontal boundary is neutral. In the following, we aim at identifying a parameter region where the formation of the targeted structure is promoted by systematically optimizing the length, $L_{W}$, of the neutral portion of the vertical side wall and the width, $\mathrm{L}_{X}$, of the confinement. The design space is restricted to the interval $1.9 \leqslant \mathrm{~L}_{X} / \mathrm{L}_{0} \leqslant 2.16$, illustrating the role of the mismatch between the width of the confinement and the equilibrium lamellar periodicity, and $\frac{1}{2} \leqslant \mathrm{~L}_{W} / \mathrm{L}_{0} \leqslant 2$. The latter quantity controls the 
number and location of horizontal lamellae at the top.

The final morphology, into which the dynamics converges according to Eq. (2.32), sensitively depends on the geometry of the confinement and the guiding pattern. The outcome of the simulations of the Ohta-Kawasaki model in this twodimensional design space are compiled in Fig. 3.6. The metastable structures encompass a variety of structures including T-junctions of $\mathrm{A}$ and $\mathrm{B}$ domains. Some general trends can be straightforwardly identified: (i) The larger $\mathrm{L}_{W}$ is the larger is the number of horizontal lamellae at the top. Note that half an A domain of thickness $\mathrm{L}_{0} / 4$ is formed at the A-attracting guiding pattern at the top. For $\mathrm{L}_{w} / \mathrm{L}_{0} \leqslant 1$ we often observe the formation of 1.5 horizontal $A$ domains at the top, whereas for $1.25 \leqslant \mathrm{~L}_{W} / \mathrm{L}_{0}$ the number of horizontal $A$ domains increases to 2.5. (ii) For $\mathrm{L}_{W} / \mathrm{L}_{0} \approx 0.75$ and 1.75, the horizontal lamellae are rather straight because the surface pattern registers the $A B$ interface of the lowest, horizontal $A$ domain at a position, $y$, that is compatible with the equilibrium lamellar spacing. For other values of $L_{w}$ the lowest, horizontal $A$ domain bulges upwards, $\mathrm{L}_{W} / \mathrm{L}_{0}=1$, or is bent downwards, $\mathrm{L}_{W} / \mathrm{L}_{0}=1.25$ and 1.5. (iii) In the range of widths, $\mathrm{L}_{X} / \mathrm{L}_{0} \approx 2$, typically half a vertical $A$ domains forms at the left and right, $A$-attractive, vertical boundaries, and an additional vertical A domain is located at the center. The horizontal domains at the top and the vertical domains at the bottom join in a grain-boundary-like structure.

For a wide parameter range we observe a U-shaped B domain, formed by connecting the two neighboring, vertical B domains at the top, where they meet the horizontal lamellae.

The A domains form the desired single T-junction at the center in the rather narrow parameter regime, $\mathrm{L}_{W} / \mathrm{L}_{0}=1.75$ and $2.06 \leqslant \mathrm{~L}_{X} / \mathrm{L}_{0} \leqslant 2.10$. The morphologies are indicated by green frames in Fig. 3.6. Apparently, the slightly wider system size, $\mathrm{L}_{X} / \mathrm{L}_{0}>2$, allows for the bulging at the ends of the $A$ domains, which are located at the vertical boundaries, without pinching-off the central, vertical A-domain of the T-junction.

Another type of T-junction of $A$ domains involves the two vertical $A$ domains at the boundary (black frames in Fig. 3.6), and it is observed for $1.25 \leqslant$ $\mathrm{L}_{w} / \mathrm{L}_{0} \leqslant 1.5$ at $\mathrm{L}_{x} / \mathrm{L}_{0}=1.96$ and $1.75 \leqslant \mathrm{~L}_{w} / \mathrm{L}_{0} \leqslant 2$ at $\mathrm{L}_{x} / \mathrm{L}_{0}=2$.

T-junctions of $B$ domains are fabricated at $\mathrm{L}_{W} / \mathrm{L}_{0}=0.5$ at $\mathrm{L}_{x} / \mathrm{L}_{0}=2$ and $\mathrm{L}_{W} / \mathrm{L}_{0}=1.75$ at $\mathrm{L}_{x} / \mathrm{L}_{0}=2.16$. These morphologies are indicated by yellow frames in Fig. 3.6.

At the extreme parameter values, more complex morphologies are formed. For $\mathrm{L}_{X} / \mathrm{L}_{0} \geqslant 2.06$ and $\mathrm{L}_{W} / \mathrm{L}_{0} \leqslant 1.25$ there is the possibility that the vertical A domain at the center breaks up into two circular domains and for $\mathrm{L}_{X} / \mathrm{L}_{0} \leqslant$ 1.96 and $\mathrm{L}_{W} / \mathrm{L}_{0} \geqslant 1.75$ highly bent structures or morphologies with additional horizontal A domains are formed. 


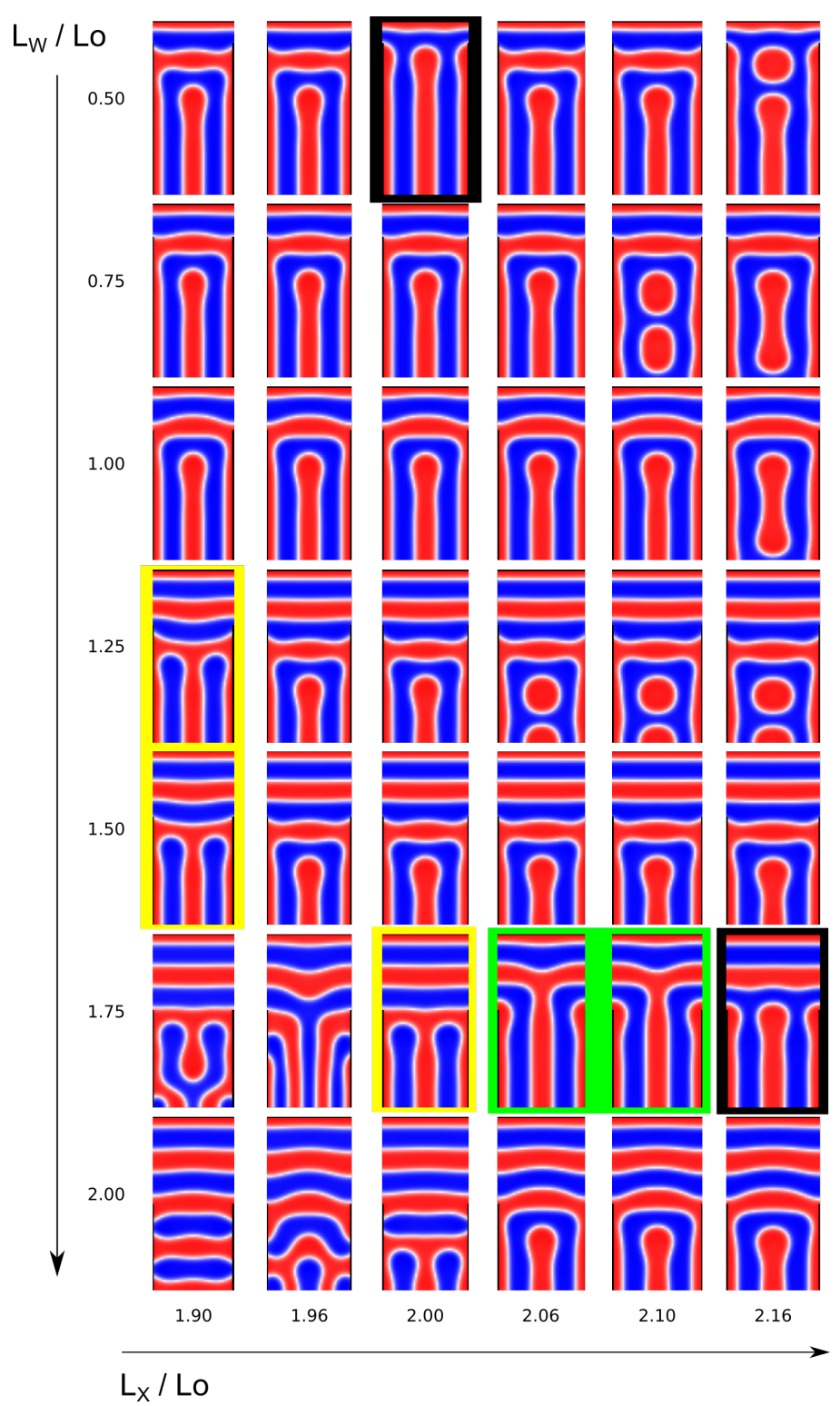

Figure 3.6: Representative set of final, metastable morphologies obtained by DSA from the disordered state as a function of the two design parameters, the width $\mathrm{L}_{X}$ of the confinement and the geometry, $\mathrm{L}_{W}$, of the guiding pattern. A domains are colored red, whereas B domains are depicted in blue. The geometry of the A-attractive guiding pattern at the boundaries is indicated by black lines. Green frames indicate the desired single T-junctions of the A species. Tjunction of $A$ domains involving the two vertical $A$ domains at the boundary are highlighted by black frames. T-junctions of $B$ domains are marked by yellow frames. 
In Fig. 3.7 we illustrate the kinetics of structure formation after a quench from the disordered state, $\mathrm{m}(\mathbf{x}) \equiv 0$, for the formation of a U-shaped B domain that occurs at $\mathrm{L}_{W}=0.75 \mathrm{~L}_{0}$ and $\mathrm{Lx}_{x}=2.0 \mathrm{~L}_{0}$. The evolution of self-assembly is directed by the guiding fields.

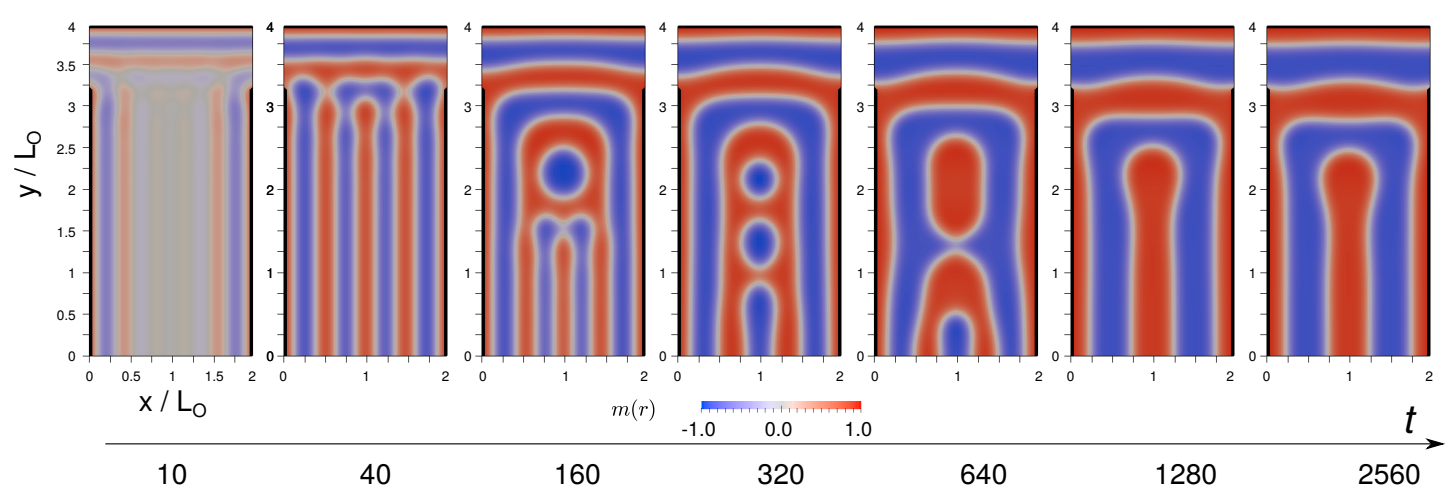

Figure 3.7: Snapshots of the evolution of structure formation of a U-shaped B domain for $\mathrm{L}_{W}=0.75 \mathrm{~L}_{0}$ and commensurate system width, $\mathrm{L}_{X}=2.0 \mathrm{~L}_{0}$. The color map indicates the order-parameter field, $m(\mathbf{x})$. A domains are depicted in red, $B$ domains are colored blue. The A-attractive guiding pattern is indicated by black lines.

At $t=10$ we observe the initial stage - surface-directed spinodal self-assembly. The guiding pattern has given rise to the formation of weakly segregated $A$ enrichment layers at the preferential portions of the boundaries according to Eq. (3.4). These low-amplitude composition variations are exponentially amplified by the self-assembly process in the unstable homogeneous state. This surface-directed spinodal self-assembly results in the formation of lamellar domains or order-parameter waves parallel aligned with the preferential boundaries, i.e., from the horizontal top and the two vertical sides, lamellae propagate towards the center of the system. Importantly the characteristic periodicity is set by the wavelength, $\lambda_{\max }$, of the fastest growing composition mode, cf. Eq. (3.5), and it is significantly smaller than the equilibrium domain spacing, $\mathrm{L}_{0}$, i.e., in a system of width $L_{X}=2 L_{0}$ we observe the initial formation of 5 vertical lamellae with rather small amplitude of the order parameter.

Subsequently, at the end of the surface-directed spinodal self-assembly $t=$ 40 , the composition inside the domains attains it saturation value. Note also that the two central, vertical A domains have already merged so that only 4 vertical A domains remain - twice than in the final, metastable state at $\mathrm{L}_{X}=2 \mathrm{~L}_{0}$.

In the later stage, $t \geqslant 160$, the domain morphology changes as to obtain a stress-free morphology with a near-equilibrium spacing. The snapshots at 
$t=160,320$ and 640 illustrate that this process is rather complex, involving the merging of domains and motion of the internal $A B$ interfaces that resembles the motion of apposing dislocation defects with impact parameter, $\Delta=0$ (evaporation mechanism). The details of these domain changes depend how the order-parameter waves emanating from the A-attractive boundaries interfere. This interference, in turn, is dictated by the design parameters, $\mathrm{L}_{W}$ and $\mathrm{L}_{x}$. Whereas the spinodal stage lasts for 40 time units of the Ohta-Kawasaki model (using $\Lambda=1$ ), the subsequent changes of the morphology take about 32 times longer.

Only at $t=1280$ the final domain topology is achieved and the subsequent kinetics merely optimizes the detailed position of the internal $A B$ interfaces. Thus, a significant fraction of the time it takes to obtain the final morphology is devoted to the changes of the domain morphology required by the mismatch of the initial periodicity, $\lambda_{\max }$, and the equilibrium domain spacing, $\mathrm{L}_{0}$. Given the use of a composition-independent Onsager coefficient, we expect that the kinetics of structure changes would be even slower if we duly accounted for the intrinsically slow "tunneling" of blocks across domains of the opposite species.[64]

In order to illustrate the qualitative universality of the different stages and the complexity of the changes of domain topology, we investigate in Fig. 3.8 the case, where the width, $\mathrm{L}_{X}=1.9 \mathrm{~L}_{0}$, is not commensurate with the equilibrium domain spacing, using two different values of $\mathrm{L}_{W}$.

For $\mathrm{L}_{W}=1.5 \mathrm{~L}_{0}$, the surface-directed, spinodal self-assembly at the bottom is similar to Fig. 3.7. Short-wavelength order-parameter waves emanate from the two vertical, A-attractive guiding patterns, yielding 4 vertical $A$ domains at the end of the spinodal self-assembly, $t=40$. Since the neutral portion, $\mathrm{L}_{W}$ at the top of the vertical boundaries is larger than in Fig. 3.7 the horizontal domain structure directed by the top boundary propagates farther downwards. Thus the transient morphology at the end of the spinodal self-assembly, $t=$ 40, features one more horizontal A domain than in Fig. 3.7, and the grain boundary where the vertical and horizontal domains meet differs, resulting in an alternate sequence of domain changes in the subsequent ordering, $160 \leqslant t \leqslant$ 22 000. Again, the domain topology changes in order to establish domains of the preferred dimension, $\mathrm{L}_{0}$. These changes involve the downward expansion of the two horizontal A domains at the top and the evaporation-like elimination of a vertical $B$ domain at the center. The final structure consists of a T-junction of $A$ domains that involves the two vertical $A$ domains at the boundary. Note that the second stage - changes of the domain topology - takes about an order of magnitude longer than in the example of Fig. 3.7, before the final metastable morphology is attained. 
(a) $L_{W}=1.50 L_{0}$

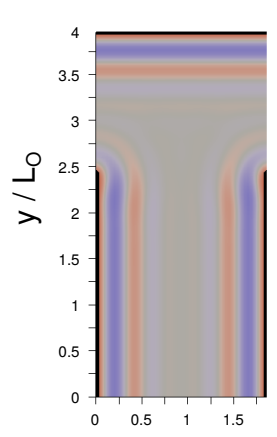

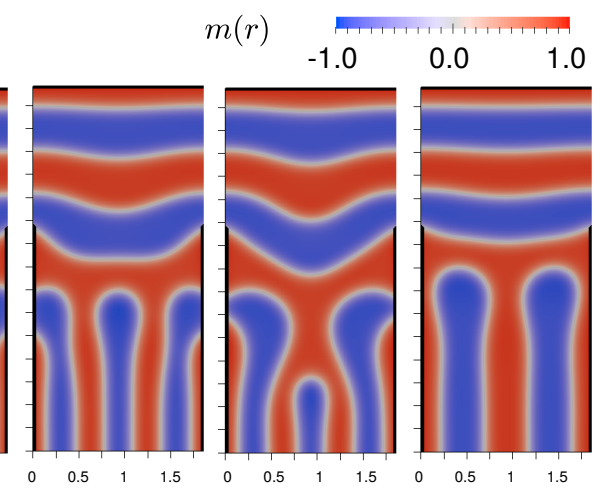

(b) $\mathrm{L}_{\mathrm{w}}=\mathbf{2 . 0} \mathrm{Lo}_{\mathrm{o}}$

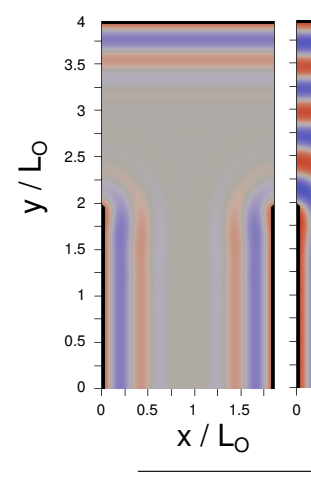

10

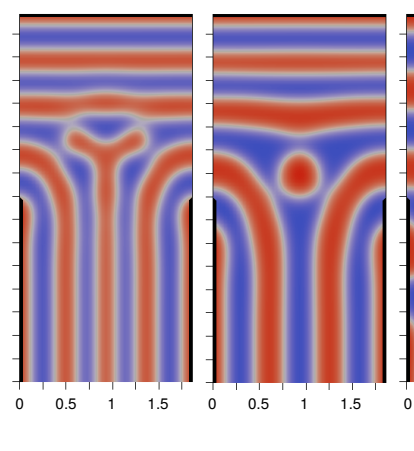

40
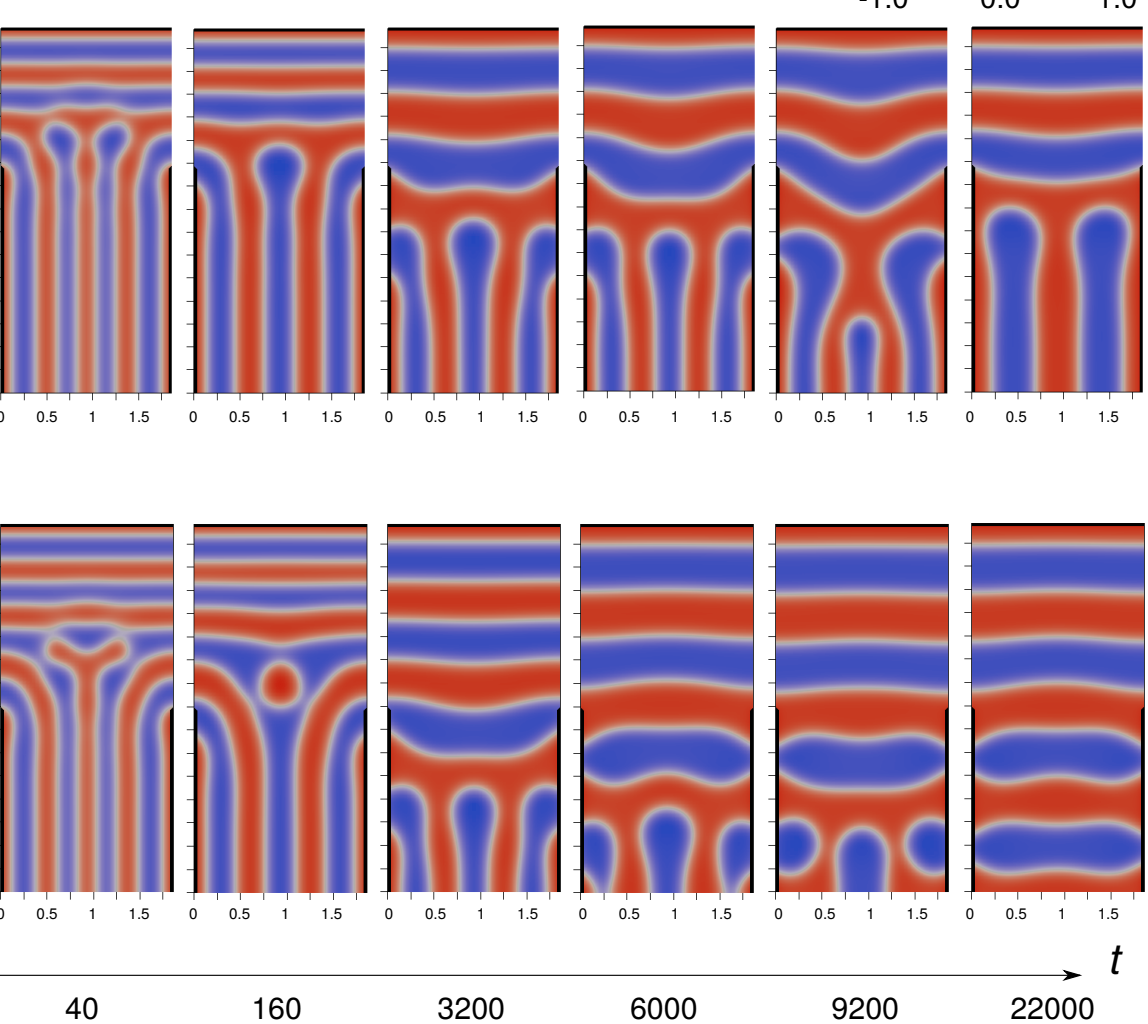

Figure 3.8: Comparison of the evolution of structure formation for two different values of $\mathrm{L}_{W} / \mathrm{L}_{0}=1.5$ and 2 in a confinement of incommensurate width, $\mathrm{L}_{X}=$ $1.90 \mathrm{~L}_{0}$. The color map indicates the order-parameter field, $m(\mathbf{x})$. A domains are depicted in red, B domains are colored blue. The A-attractive guiding pattern is indicated by black lines.

For $\mathrm{L}_{W}=2 \mathrm{~L}_{0}$, an additional horizontal $A$ domain is generated by the surfacedirected spinodal self-assembly at $t=40$ and, concomitantly, the vertical A domains at the bottom are less extended upwards. The subsequent changes of the domain topology, involving highly curved $A B$ interfaces, completely eliminate the vertical domains (without any barrier). Presumably, the free-energy costs of (i) slightly compressing the vertical lamellae for $\mathrm{L}_{X}<2 \mathrm{~L}_{0}$ and of (ii) the grain boundary between vertical and horizontal domains outweigh the cost of deforming the left and right ends of the two horizontal domains in contact with the A-attractive boundaries at the lower part of the vertical boundaries. As for $\mathrm{L}_{W}=1.5 \mathrm{~L}_{0}$, the stage of changing the domain topology is protracted.

In all these examples, most of the time is spent on the stage of changing the domain topology after the surface-directed spinodal self-assembly has tem- 
plated the initial morphology. The change of the domain topology is chiefly required because the initial morphology that is templated by the surface-directed spinodal self-assembly differs in characteristic length scale, $\lambda_{\max }$, from the domains spacing, $\mathrm{L}_{0}$, in equilibrium. Since the ratio between the two length scales differs from unity the more, the stronger the segregation strength is, cf. Eq. (3.7), DSA at weak segregation, $10.5<\chi \mathrm{N}<20$, appears to be advantageous for rapid self-assembly directed by guiding patterns into device-oriented structures.

\subsubsection{Spinodal length scale}

In the previous section we presented how the ordering process is influenced by two characteristic periodicities in DSA: the wave-length of the fastest growing mode $\lambda_{\max }=8.886$ (see Eqn. 3.6) and the equilibrium domain spacing $\mathrm{L}_{\mathrm{O}}$. Three stages during the ordering process were identified: (i) an surface-directed spinodal self-assembly which results in an initial morphology templated by the periodicity $\lambda_{\max }$, (ii) an intermediate domain-merging stage that results in the final topology and (iii) a final equilibration stage where the internal interfaces of the established final topology optimize their detailed positions. The intermediate domain-merging process is the result of the mismatch between the periodicities $\lambda_{\max }$ and $\mathrm{L}_{0}$.

It is intuitive to design a confinement commensurate to the equilibrium lamellar period $\mathrm{L}_{0}$, however, using $\lambda_{\max }$ as a design scale length we can control and tailor the kinetics of structure formation. If the size of the confinement and the process conditions can be tuned such that the ordered structure formed at early times becomes metastable, the domain-merging stage can be omitted or reduced such that a faster structure formation takes place. On the other hand, if the mismatch between the two length scales $\lambda_{\max }$ and $L_{0}$ is reduced, the domains formed after the initial step do not need to undergo substantial changes, hence a structure formation with no merging process could be obtained as well.

The domain-merging stage not only protracts the time required to obtain the final structure but also determines how prone is the system to the formation of undesired domains with respect to the targeted structure. Therefore reducing or eliminating the merging stage could reduce the defectivity of the final metastable structures fabricated via DSA.

This section is devoted to explore these ideas in more detail. Here we investigate how the mismatch between those length scales can be exploited to control the guided self-assemble process in two cases: the structure formation of a thin film between two guiding walls and the control of the formation of the intermediate checkerboard structure in thin films as well. 


\section{DSA of thin films via symmetric guiding walls}

For the first case, the structure formation between two guiding walls, we employ a two-dimensional system with reflective boundary conditions in the $X$ direction mimicking confining walls and periodic boundary conditions in the $Y$ direction representing a periodic thin-film. As in previous calculations, we ignore thermal fluctuations by starting from a perfectly homogeneous state, $m(\mathbf{x}) \equiv 0$, and in our deterministic kinetics we focus on how the guiding surfaces direct the self-assemble process. Snapshots of the system are presented in Figure 3.9.
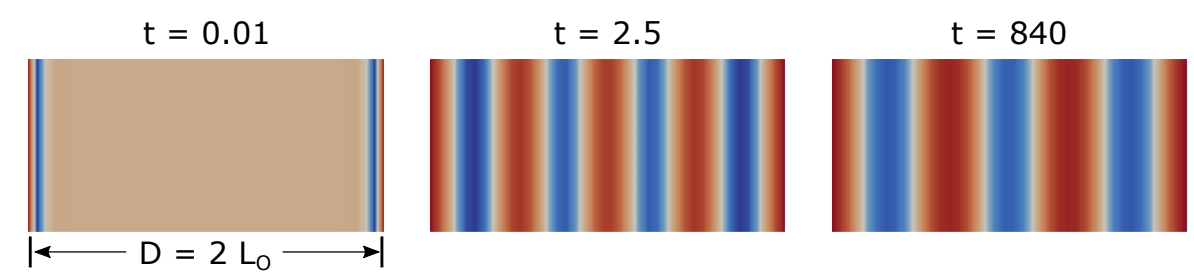

Figure 3.9: 2D calculations via the Ohta-Kawasaki model to investigate the final morphology and the different stages of the surface-directed structure formation. The guiding pattern consists of $A$ - attractive vertical boundaries at the two sides of the film of thickness $D$, which attract the A-component with the same strength of $h(0, y)=h(D, y)=-0.3$. Snapshots correspond to $D=2 L_{O}$ at $\chi \mathrm{N}=30\left(\lambda_{\max } / \mathrm{L}_{\mathrm{O}}=0.51\right)$. Red and blue correspond to A- and B-domains respectively.

In the following, we aim to compare the final morphology obtained for different values of the mismatch ratio, $\lambda_{\max } / \mathrm{L}_{\mathrm{O}}$, as well as to describe the differences in the structure formation process. To this end, two values of $\chi \mathrm{N}$ are selected to tune the mismatch ratio: $\chi \mathrm{N}=30$ for which $\tilde{\alpha}=0.0172, \mathrm{~L}_{\mathrm{O}}=18.280$ and $\lambda_{\max } / \mathrm{L}_{\mathrm{O}}=0.49$, i.e., significant mismatch where about two wavelentghs of the fastest growing mode can be allocated in the length given by one equilibrium domain spacing, and $\chi \mathrm{N}=11$ for which $\tilde{\alpha}=0.2080, \mathrm{~L}_{\mathrm{O}}=9.362$ and $\lambda_{\max } / L_{O}=0.95$, i.e., virtually no mismatch. We study values of the film thickness commensurate to the natural domain spacing $\mathrm{D} / \mathrm{L}_{\mathrm{O}}=2,3,4$. In every case, the height of the system if fixed as $L_{Y}=L_{O}$ deployed in 20 grid cells.

In Figure 3.10 we present the order-parameter profile of the final morphology for every value of the film-thickness and the mismatch ratio. Regardless of the film-thickness, equilibrium morphologies commensurate to the film-thickness are obtained when the mismatch ratio is virtually $1, n=D / L_{O}=2,3,4$. Given the similarity between the two periodicities, the lamellar structure formed at the end of the initial surface-directed spinodal self-assembly is virtually the same 
as the final equilibrium configuration templated by $\mathrm{L}_{\mathrm{O}}$, and the subsequent kinetics merely optimizes the detailed position of the internal AB interfaces.

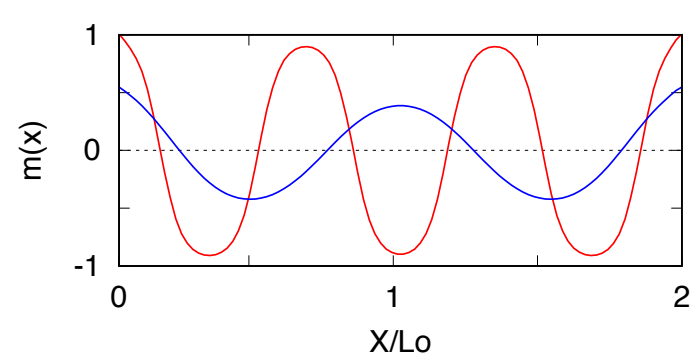

$$
\begin{aligned}
& \chi N: 30 \\
& \lambda_{\text {max }} / \mathrm{L}_{\mathrm{O}}=0.49 \\
& \chi \mathrm{N}: 11 \\
& \lambda_{\max } / \mathrm{L}_{\mathrm{O}}=0.95
\end{aligned}
$$
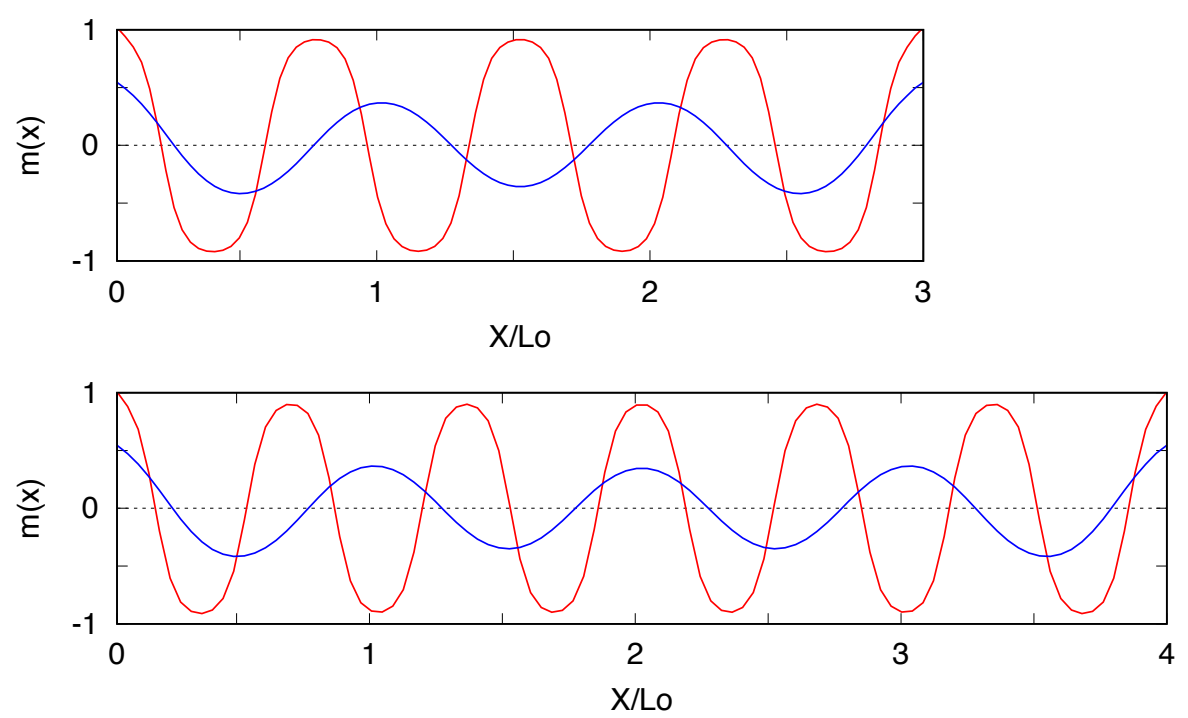

Figure 3.10: Equilibrium lamellar topology at two different values of $\lambda_{\max } / \mathrm{L}_{\mathrm{O}}$. At virtually no mismatch, $\lambda_{\max } / \mathrm{L}_{\mathrm{O}} \approx 1$, the number of lamellar domains in the final morphology, $n$, is commensurate to the film-thickness, $n=D / L_{O}=$ $2,3,4$ (blue). In contrast, additional domains are obtained when the mismatch is significant $\lambda_{\max } / \mathrm{L}_{\mathrm{O}}=0.49, \mathrm{n}=3,4,6$ (red) for $\mathrm{D} / \mathrm{L}_{\mathrm{O}}=2,3,4$ respectively.

In contrast, when the mismatch ratio is $0.49, \chi N=30$, the formation of additional lamellar domains indicates the final configuration corresponds to a metastable structure. In this case, several domains need to merge in the course of the ordering process, as a consequence of the mismatch between the two periodicities, changing the number and position of the internal $A B$ interfaces. During this merging process, the system can find a local minimum in the free-energy landscape that corresponds to a different number of domains as can be expected by conmmensurability of the film-thickness with respect to the equilibrium periodicity $\mathrm{L}_{\mathrm{O}}$. This is the case for the final states obtained for $\lambda_{\max } / L_{O}=0.49$ where the domains are compressed, and hence, the system 
does not correspond to the global minimum of the free energy. This can be illustrated by the comparison of the free energy of the final state for $\mathrm{D} / \mathrm{L}_{\mathrm{O}}=4$, which contains 6 lamellar domains, with a fabricated state that contains 4 lamellar domains commensurate to that value of $\mathrm{D}$. The free-energy density for the state with 6 domains is $f=-0.0806$, whereas for the state with 4 domains is $f=-0.1025$, confirming the final configuration depicted in Fig. 3.10 (see bottom red plot) corresponds to a metastable state resulting from the mismatch ratio.

In addition, the duration of the initial spinodal stage and the time to achieve the final topology differs as the mismatch ratio changes. The surface-directed spinodal stage ends once the two composition waves, prompted by the lateral guiding patterns, interfere and give rise to an initial lamellar pattern. The subsequent kinetics are determined by the mismatch ratio and the film thickness. For the case of a mismatch ratio of 0.95 , the final topology is obtained at $t=100$ in time units of the Ohta-Kawasaki model (using $\Lambda=1$ ) and the spinodal stage ends at $t=75$, i.e, the formation of the final topology takes 1.3 longer. For the mismatch ratio of 0.49 those times are $t=270$ and $t=50$ respectively and thus the formation of the final structure is protracted to 5.4 times the duration of spinodal stage. The faster spinodal stage for the higher incompatibility can be analyzed by means of the dynamics of the micro-phase separation in Eqn. 2.32

$$
\frac{\partial \mathrm{m}}{\partial \wedge \mathrm{t}}=\quad \Delta\left(-\mathrm{m}+\gamma \mathrm{m}^{2}+\mathrm{m}^{3}-\Delta \mathrm{m}\right)-\alpha \mathrm{m}
$$

At higher incompatibility, lower value of $\alpha$, changes in local composition occur faster in comparison to lower values of $\chi \mathrm{N}$. Therefore, is reasonable to expect a shorter spinodal stage at $\chi \mathrm{N}=30$, mismatch ratio of 0.49 , compared to the value at $\chi \mathrm{N}=11$, mismatch ratio of 0.95 , as shown in our findings.

In order to describe the ordering process in more detail, including the aforementioned merging stage, we analyze the evolution of the composition profile along the formation process for a larger film, $\mathrm{D}=12 \mathrm{~L}_{\mathrm{O}}$, under the same guiding fields and the same initial homogeneous state. In the initial stage, composition changes are promoted by the guiding effect of the lateral surfaces until a saturated lamellar domain is formed, i.e., surface-directed self-assembly. During this stage, the maximum composition is located at the vicinity of the guiding surface and it grows linearly in time, as shown in Figure 3.11. In contrast to the exponential composition changes during spinodal decomposition in the bulk, the kinetics of the structure formation in DSA is dictated by the interplay between the linear composition growth prompted by the guiding surface and the exponential composition growth taking place in the bulk. The present calculations do not consider the exponential composition changes given the choice to 
omit order-parameter fluctuations in the initial state to focus on the effect of the guiding pattern in the ordering process.

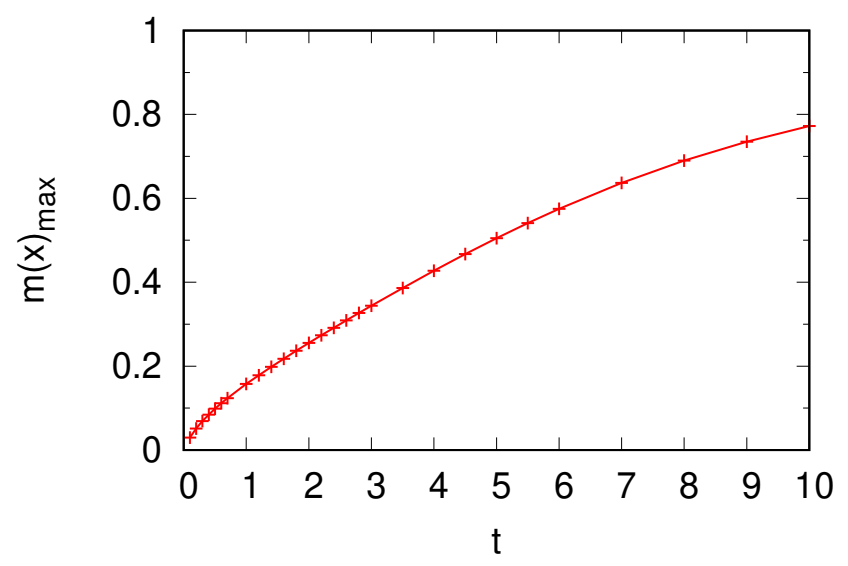

Figure 3.11: Growth of the maximum composition $m(x)$ during the surfacedirected spinodal self-assembly from a disorder state $m(x) \equiv 0$.

After the initial linear composition growth at side wall, the newly formed domain at time $t=10$ acts as a guiding surface promoting composition changes in the neighboring block copolymers, leading to the formation of the next saturated domain and the propagation of the interface between a lamellar domain at the guiding surface and a disordered domain in the bulk. Given the symmetry of the guiding pattern composed of two lateral surfaces with the same A-preference strength, the two composition undulations are expected to have the same profile and to propagate at the same speed until they interfere. In Figure 3.12 we present the composition profiles at different times during the ordering process, where the expected symmetry in the profiles shows the proper accuracy of the numerical calculations. As the surface-directed spinodal decomposition stage ends, $t=100$, a lamellar topology with 26 domains is established, that corresponds to more than 2 times the number of domains with the natural spacing expected from the film thickness of $\mathrm{D}=12 \mathrm{~L}_{\mathrm{O}}$. This indicates that the lamellar domains are compressed and the free energy of the system is higher than the corresponding value at the equilibrium state with 12 domains, hence, this difference drives the following changes. The 26 domains in this initial lamellar structure have slight changes in the saturation values; beside the domains at the vicinity of the surface, the domain in the middle of the film $\left(\mathrm{X} / \mathrm{L}_{\mathrm{O}}=6\right)$ possesses the maximum composition amplitude which remains unaltered after its formation at $t>100$. Therefore, this middle domain can be conceived as a neutral wall.

To decrease the free energy of the system, changes in the compressed lamel- 

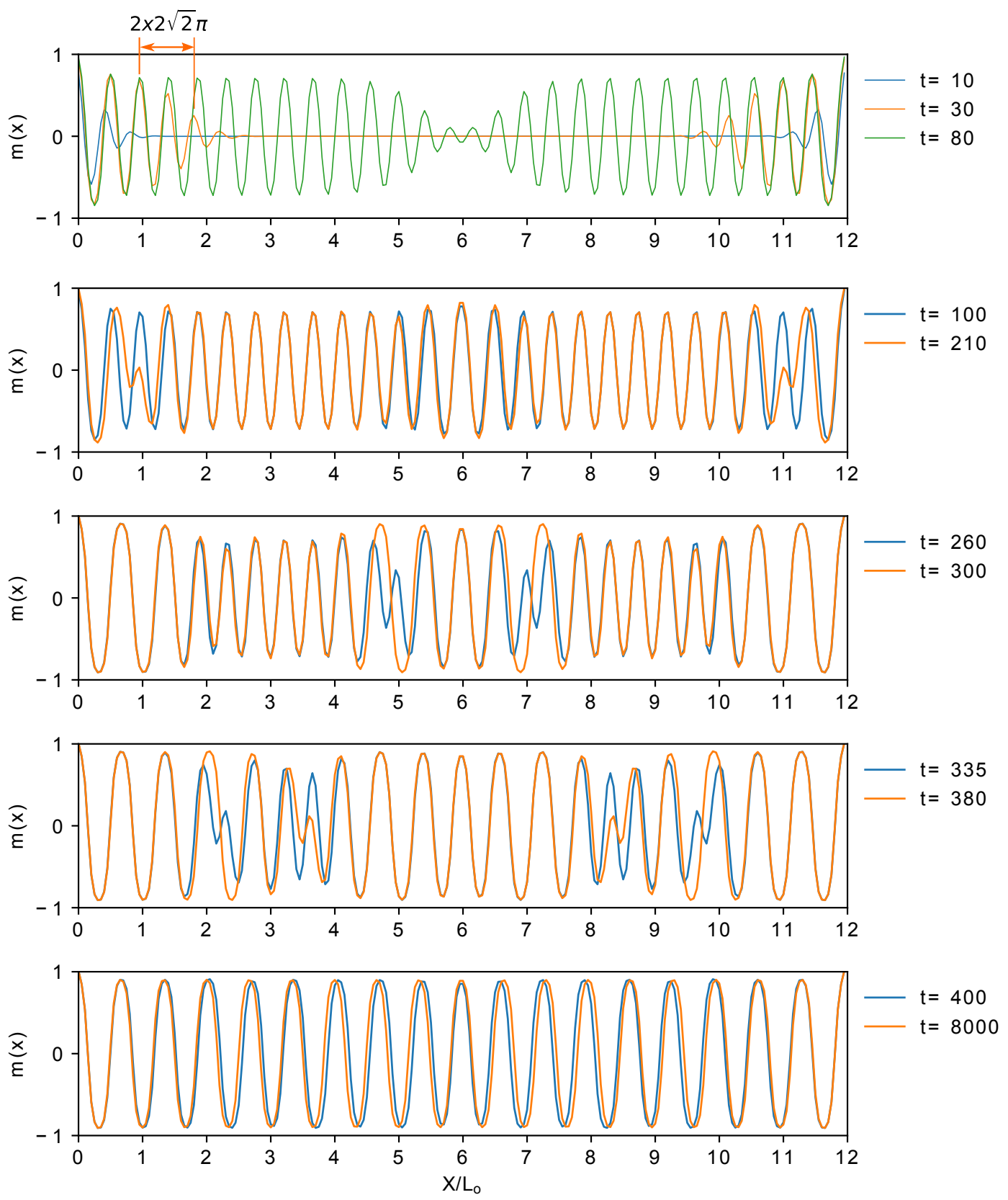

Figure 3.12: Evolution of the order-parameter profile during the structure formation at $\chi \mathrm{N}=30\left(\lambda_{\max } / \mathrm{L}_{\mathrm{O}}=0.49\right)$ for a film thickness, $\mathrm{D}=12 \mathrm{~L}_{\mathrm{O}}$. The number of lamellar domains evolves from $n=26$, at the end of the surface-directed spinodal self-assembly at $t=100$, to $n=18$ when the final topology is achieved at $t=400$. Profiles at $t=210,260,335,380$ illustrate the merging of domains and the motion of the $\mathrm{AB}$ interfaces driven by the mismatch between the two periodicities $\lambda_{\max }=2 \sqrt{2} \pi$ and $\mathrm{L}_{\mathrm{O}}$. 
lae start at $t=210$ when two lamella next to the guiding surface merge reducing the number of domains located at $10 \leqslant X / L_{O} \leqslant 12$ from 4 at $t=100$ to 3 at $t=260$. At $t=260$, a similar process takes place next to the middle domain, $6 \leqslant X / L_{O} \leqslant 8$, reducing the number of domains in that region from 4 at $t=210$ to 3 at $t=300$. As a result, a new intermediate topology with 22 domains is formed at $t=300$. Four domains merge in the following stages, $t=210$ and $t=300$, in the region between the guiding field and the middle domain, $8 \leqslant X / L_{O} \leqslant 10$, giving rise to a final structure with 18 domains at $t=400$. After this time, the kinetics merely optimizes the detailed position of the internal interfaces and no further merging of domains leading to the predicted lamella with 12 domains occur. As previously mentioned, the mismatch ratio far from the value of 1 gives rise to a final metastable state with additional domains with respect to the prediction based on the value of the film thickness commensurate to the natural spacing.

\section{Control of the checkerboard-structure formation}

When the assembly of lamellae-forming block copolymers is guided by a pattern of stripes, the formation of an undesired intermediate structure known as checkerboard is a typical problem. Therefore, we investigate how the mismatch between the length-scales of spinodal decomposition and final structure can be exploited in order to promote or suppress the formation of the checkerboard structure at intermediate times.

To this end, we performed simulations with two different values of the film thickness and study if the initial composition variations can be altered in order to influence the formation of the undesired intermediate morphology. In our $2 \mathrm{D}$ calculations we use a symmetric guiding pattern of width $\mathrm{L}_{x}=2.0 \mathrm{~L}_{0}$ consisting of alternating A- and B- attractive stripes of widths $W=\mathrm{L}_{0} / 2$. The initial configuration is a perfectly homogeneous state, $\mathrm{m}(\mathbf{x}) \equiv 0$, at time, $t=0$, and we use $\tilde{\alpha}=0.0817$ corresponding to $\chi N \approx 16$. We optimize the domain spacing with respect to the free energy of the system, therefore $\mathrm{L}_{0}$ slightly differs from the one-mode approximation. The periodicity, $\lambda_{\max }$, set by the wavevector corresponding to the maximal growth rate $\mathrm{q}_{\max }$ and the equilibrium domain spacing, $\mathrm{L}_{0}$, obeys $\lambda_{\max } / \mathrm{L}_{0} \approx 0.75$. We consider film thicknesses $\mathrm{D}=0.75 \mathrm{~L}_{0}=\lambda_{\max }$ and $\mathrm{D}=\mathrm{L}_{0}=1.3 \overline{3} \lambda_{\max }$ and the corresponding kinetics of structure formation is shown in Figure 3.13.

During the initial stages, $t \leqslant 10$, the spinodal wave templates the formation of two horizontal grain boundaries, i.e., checkerboard morphology with 3 cells in the vertical direction is formed. The composition inside each cell has not reached its equilibrium saturation value. If this initial composition wave reached saturation, a checkerboard morphology with periodicity $\lambda_{\max }$ would 


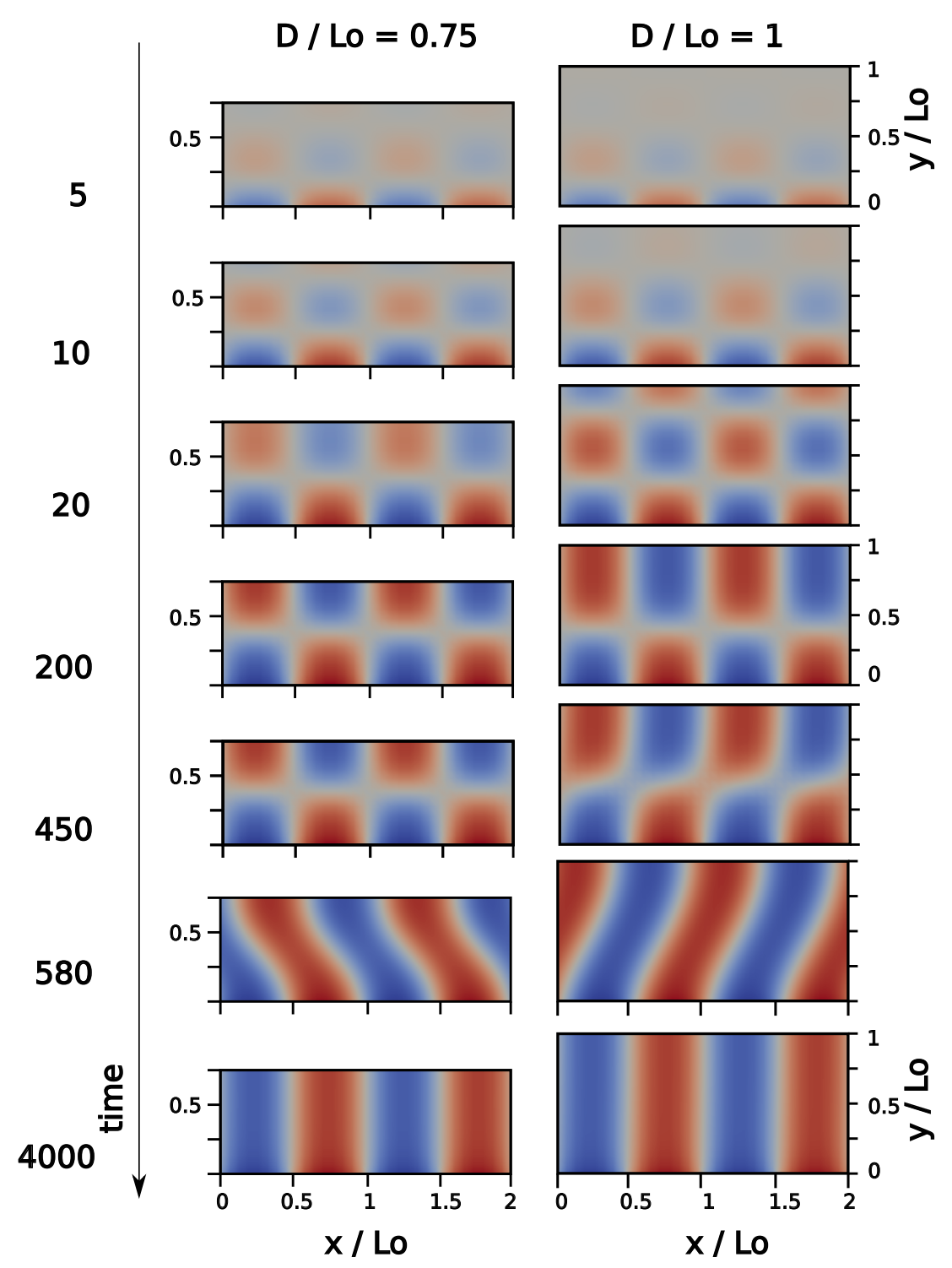

Figure 3.13: Comparison of the checkerboard structure formation at two values of the film thickness $\mathrm{D} / \mathrm{L}_{0}=0.75$ and 1.0 using $2 \mathrm{D}$ calculations with the Ohta-Kawasaki model. Snapshots of the morphology show the formation of the horizontal grain boundary at the onset of the surface-directed spinodal decomposition, $t=5$. This grain boundary gradually moves upward away from the bottom patterned surface, $t \leqslant 20$. A coarser intermediate checkerboard structure is formed and shortly lives for $200 \leqslant t \leqslant 450$. For the case of $D / L_{0}=1$ this period of time is slightly shorter than for $\mathrm{D} / \mathrm{L}_{0}=0.75$. The final lamellar structure arises after the domains merge diagonally and the top grain is shifted to align with the bottom domain. 
form, potentially leading to a metastable, highly undesired state. However, both horizontal grain boundaries gradually move upwards away from the bottom patterned surface, and the second one, which is closer to the top of the film, vanishes. An intermediate checkerboard with only one horizontal grain boundary is formed at $t=20$. In fact the thickness of the bottom cell grows even larger than the equilibrium value $\mathrm{L}_{0} / 4$, indicating that the aligned and registered grain wets the guiding pattern. This observation is in accord with particle-based simulations [92]. The saturation of the order parameter inside the checkerboard cells is reached in this coarser morphology - an undesired structure that delays the formation of defect-free, standing lamellae. Since the spinodal wave does not directly template the formation of this intermediate checkerboard, we cannot immediately take advantage of the fastest growing mode, $\lambda_{\max }$, to tailor the morphology at intermediate times.

Regardless of the film thickness, the checkerboard structure evolves into the standing lamella. At $t=450$ the horizontal grain boundary remains mainly unaltered for $\mathrm{D} / \mathrm{L}_{0}=0.75$ but $\mathrm{A}$-domains connect in the diagonal direction, giving rise to the formation of standing lamella in the case of $\mathrm{D} / \mathrm{L}_{0}=1$ via the shifting of the top grain to align with the bottom grain, see snapshot at $t=580$. The same diagonal merging of the top and bottom grain occurs for $\mathrm{D} / \mathrm{L}_{0}=0.75$ at a slightly later time such that at $\mathrm{t}=580$ both systems evolve into an intermediate diagonal lamellar structure previous to the formation of the final morphology. For $\mathrm{D} / \mathrm{L}_{0}=1$, the intermediate checkerboard annihilates faster compared to the commonly used value of $\mathrm{D} / \mathrm{L}_{0}=0.75$, which maximally frustrates lying structures in equilibrium.

Although there is an interplay between the spinodal structure formation and the morphology at intermediate times, the simulations indicate that there is no one-to-one correspondence. Since the surface fields drive the initial kinetics, the segregation is earlier reached at the bottom. Thus, the bottom part prefers a larger spacing that is closer to the equilibrium value.

The findings from the two cases presented above are in agreement with our results from the previous section, suggesting that values of $\chi N$ close to the ODT may facilitate defect-free DSA structures. Complementary insights supporting the choice of low incompatibility values for DSA are provided from the investigation of the effect of the mismatch ratio, $\lambda_{\max } / \mathrm{L}_{\mathrm{O}}$, on the kinetics of the ordering process. Faster structure formation and a system less prone to the formation of defects can be promoted by tuning the DSA characteristic dimensions to $\lambda_{\max }$ via a mismatch ratio close to the unity. 


\subsection{Summary and conclusions}

[...] We have evaluated to what extent the Ohta-Kawasaki model can capture (i) the kinetics of defect motion and (ii) the surface-directed self-assembly driven by guiding patterns. The ability of the model to describe the metastability of defects and to provide insights into the kinetics of structure formation is of special relevance to DSA applications. The computational speed allows for a systematic exploration of parameter spaces - as illustrated here for a special case of a device-oriented structure. Generally, good agreement with previous SCFT calculations and particle-based simulations is obtained, apart from the following exceptions: (i) The model commonly uses a composition-independent Onsager coefficient, $\wedge$. Computationally, this choice is very convenient for it makes the time evolution, Eq. (2.32), local. Whereas it generally captures the qualitative features of diffusive kinetics, it grossly speeds up intrinsically slow evaporation-like events where blocks have to "tunnel" through a domain of the opposite species.[64] In principle, one can account for this deficiency by using $\Lambda \sim \phi_{A} \phi_{B}$, imparting however, a considerable computational burden. (ii) Our study does not include thermal fluctuations. Thermal fluctuation effects can be included but, again, an accurate numerical implementation of the concomitant stochastic partial differential equation is computationally costly.[93] Due to the absence of thermal fluctuations the kinetics of self-assembly cannot overcome free-energy barriers. Therefore the calculations highlight barrier-free ordering processes that are preferred for rapid and reliable DSA. (iii) The long-range kernel, G, encodes the Gaussian chain conformations. In the vicinity of impenetrable surfaces, these chain conformations are perturbed $[94,95]$ and it remains to be investigated to what extent the Ohta-Kawasaki model can account for implications of perturbed chain conformations at spatial inhomogeneities.[96]

In spite of these caveats, the Ohta-Kawasaki model provides valuable insights into the kinetics of self-assembly and defect motion. This model can be used in combination with more sophisticated techniques [97-99] to optimize confinements suitable to fabricate device-oriented copolymer structures and limit the design space to the most promising parameter combination. Once prospective parameters for the geometry of the confinement and the guiding pattern are identified by the Ohta-Kawasaki model, more detailed simulation techniques like particle-based simulations or SCFT can be used to validate the predictions of this continuum model. This complementary use of continuum and more detailed models, enables us to gain a better understanding of the structure formation process, reducing the computational cost compared to more detailed simulation techniques.

One particularly important finding of our present study of DSA is the mismatch of the characteristic length scale of the initial surface-directed spinodal 
self-assembly and the equilibrium domain spacing that necessitates protracted changes of the domain morphology. This difficulty is reduced in the vicinity of the order-disorder transition, providing an additional motivation for using not too large values of the incompatibility, $\chi N$. 


\section{Chapter 4}

\section{Defect annihilation pathways}

This chapter deals with the study of free-energy barriers within a continuum model, specifically, the Ohta-Kawasaky free-energy functional and the use of an alternative approach to investigate fluctuations. This model can adequately describe the collective barrier-free dynamics of a block-copolymer system as previously discussed.

The strength of thermal fluctuations is dictated by the fluctuation-dissipation relation provided that the free-energy functional of the system, $\mathcal{F}$, is known. By considering an additional term, $\xi$, originating from the thermal fluctuations, we arrive at the familiar Cahn-Hilliard-Cook equation given by

$$
\frac{\partial \mathrm{m}(\mathbf{x})}{\partial \mathrm{t}}=\nabla \cdot\left[M \nabla \frac{\delta \mathcal{F}}{\delta \mathrm{m}(\mathbf{x})}\right]+\xi
$$

where $M$ is the mobility coefficient and $\xi$ satisfies the fluctuation-dissipation theorem, $[100]\langle\xi(\mathbf{x}, \mathrm{t})\rangle=0$ and $\left\langle\xi(\mathbf{x}, \mathbf{t}) \xi\left(\mathbf{x}^{\prime}, \mathrm{t}^{\prime}\right)\right\rangle=-2 M \nabla^{2} \delta\left(\mathbf{x}-\mathbf{x}^{\prime}\right) \delta\left(\mathrm{t}-\mathrm{t}^{\prime}\right)$. Thus, equation 4.1 is a stochastic partial differential equation (SPDE) whose solution describes the dynamics of the block-copolymer system. Nevertheless, both the analytic and numerical solutions of this equation require approximations or a high computational effort. In this regard, Bosse [100] obtained an approximate analytic expression for the structure factor of ordered lamellar phases in equilibrium using the Ohta-Kawasaky free-energy functional in equation 4.1. Recently, de la Torre et al.[101] presented a rigorous numerical method to solve the SPDE under consideration; their method, however, requires significant computational effort.

We resort to the concept of normal modes as an alternative approach for investigating small fluctuations instead of dealing with the SPDE given by equation 4.1. Our approach involves the solution of a non-stochastic partial differential equation, the Cahn-Hilliard equation, coupled with matrix operations, 
which is less computationally costly than the solution of the SPDE (section 4.1).

To study small fluctuations around a state of interest, we can use a harmonic approximation as an accurate description of the free-energy basin of the given state $m_{\mathrm{o}}(\mathbf{x})$ that corresponds to a local minimum in the free-energy landscape. Within that description, the normal modes are the basis, or axes, of this harmonic free-energy well and they represent the directions of change of the state $m_{\mathrm{o}}(\mathbf{x})$ due to fluctuations in that well. The normal modes provide us with a simplified method to study the various configurations arising from the fluctuations of $m_{\mathrm{o}}(\mathbf{x})$. Any possible fluctuation around the free-energy minimum is a linear combination of its normal modes, or a subset of them, which we can now also refer to as fluctuation modes. In this regard, we explore how these fluctuation modes modify a configuration of interest and provide insights about the transition towards a different state in the free-energy landscape near an energy barrier.

Furthermore, we introduce the physical background as well as the computational details required for the calculation of the fluctuation modes of a given configuration described by a continuum model. Our findings from this approach are compared with the evolution of the configuration along the minimum free-energy path obtained with a well-known technique: the string method (section 4.2).

At the end of this chapter, we exploit the features of the fluctuation modes in two applications: the identification of dominant modes in defect annihilation (section 4.3) and the prediction of the defect scattering pattern for its use in a faster initial scanning of defects in metrology (section 4.4). In the first case, local fluctuation modes describing the onset of defect annihilation are identified as the free energy landscape changes. In the second case, we identify the long-range wavevectors of the defect scattering pattern which can be observed using light scattering instead of more demanding techniques like X-ray scattering of AFM. Fluctuations modes of the lamella compete with those long-range wavevectors mask the scattering pattern of the defect. Therefore, we identify the lamellar fluctuations that contribute the most to this masking effect and predict the limiting values of $\overline{\mathcal{N}}$ where fluctuations augment that masking-effect.

\subsection{Fluctuation Mode Analysis}

In our study, we consider block-copolymer systems in the NVT ensemble and we investigate their dynamics in terms of the corresponding Helmholtz freeenergy landscape. The access to the free-energy functional, representing the relevant features of our specific block copolymer system, allows us to determine when a given configuration is a metastable, stable or unstable state. 
Free-energy fluctuations around a given metastable or stable state, $m_{0}(\mathbf{x})$, can be studied by observing the response of that equilibrium configuration to perturbations, $\delta \mathfrak{m}(\mathbf{x})=\mathrm{m}(\mathbf{x})-\mathrm{m}_{\mathrm{o}}(\mathbf{x})$. In this regard, the free energy of a configuration $m(\mathbf{x})$, i.e., $\mathcal{F}[m(\mathbf{x})]$, whose free-energy fluctuates around the corresponding metastable state can be expressed as the following Taylor expansion.

$$
\begin{aligned}
\mathcal{F}[\mathrm{m}(\mathbf{x})]= & \mathcal{F}\left[\mathrm{m}_{\mathrm{o}}\right]+\int \mathrm{d} \mathbf{x}\left(\left.\frac{\delta \mathcal{F}}{\delta \mathrm{m}(\mathbf{x})}\right|_{\mathrm{m}_{\mathrm{o}}} \delta \mathrm{m}(\mathbf{x})\right) \\
& +\frac{1}{2} \int \mathrm{d} \mathbf{x} \mathrm{d} \mathbf{x}^{\prime}\left(\left.\frac{\delta^{2} \mathcal{F}}{\delta \mathrm{m}(\mathbf{x}) \delta \mathrm{m}\left(\mathbf{x}^{\prime}\right)}\right|_{\mathrm{m}_{\mathrm{o}}} \delta \mathrm{m}(\mathbf{x}) \delta \mathrm{m}\left(\mathbf{x}^{\prime}\right)\right)+\cdots
\end{aligned}
$$

Provided that we deploy conserved Cahn-Hilliard dynamics, or model B, to compute the equilibrium configurations via the continuum model, this imposes a constraint on the perturbations, $\int \mathrm{d} \mathbf{x} \delta \mathrm{m}(\mathbf{x})=0$, such that the concentration is conserved.

For a system in thermal equilibrium, the concentration current $\mathfrak{j}^{\mathrm{m}}=-\boldsymbol{M} \nabla \mu$, must be zero, where $M$ denotes the transport coefficient. Consequently, the gradient of the chemical potential must be constant, i.e.,

$$
\left.\mu\right|_{m_{\mathrm{o}}}=\left.\frac{\partial \mathcal{F}}{\partial \mathrm{m}}\right|_{\mathrm{m}_{\mathrm{o}}}=\hat{\mathrm{c}}
$$

Therefore, the first-order term in the expansion vanishes due to concentration conservation as:

$$
\left.\int \mathrm{d} \mathbf{x} \frac{\delta \mathcal{F}}{\delta \mathrm{m}(\mathbf{x})}\right|_{\mathrm{m}_{\mathrm{o}}} \delta \mathrm{m}(\mathbf{x})=\hat{\mathrm{c}} \int \mathrm{d} \mathbf{x} \delta \mathrm{m}(\mathbf{x})=0
$$

When small perturbations $\delta \mathrm{m}(\mathbf{x})$ are considered, the higher-order terms can be assumed to be negligible; thus, the free-energy difference, $\Delta \mathcal{F}=\mathcal{F}[\mathrm{m}]-$ $\mathcal{F}\left[\mathrm{m}_{\mathrm{o}}\right]$, can be estimated as follows:

$$
\left.\Delta \mathcal{F} \simeq \frac{1}{2} \int \mathrm{d} \mathbf{x} \mathrm{d} \mathbf{x}^{\prime} \frac{\delta^{2} \mathcal{F}}{\delta \mathrm{m}(\mathbf{x}) \delta \mathrm{m}\left(\mathbf{x}^{\prime}\right)}\right|_{\mathrm{m}_{\mathrm{o}}} \delta \mathrm{m}(\mathbf{x}) \delta \mathrm{m}\left(\mathbf{x}^{\prime}\right)
$$

The second-order derivative of the free-energy functional in the above equation corresponds to a component of the Hessian matrix of $\mathcal{F}, \mathrm{H}$; thus,

$$
\mathbf{H}\left(\mathbf{x}, \mathbf{x}^{\prime}\right)=\left.\frac{\delta^{2} \mathcal{F}}{\delta m(\mathbf{x}) \delta m\left(\mathbf{x}^{\prime}\right)}\right|_{m_{\mathrm{o}}}
$$


$\mathbf{H}$ describes the shape of the potential well corresponding to state $m_{0}(x)$ via a harmonic approximation; as a result, $\mathrm{H}$ is positive semi-definite and symmetric. Therefore, we can decompose it as $\mathrm{H}=\mathrm{E}^{\top} \mathrm{D} \mathrm{E}$ where $\mathrm{E}$ is a square matrix with the eigenvectors of $\mathrm{H}, e_{k}$ 's, and $\mathrm{D}$ is a diagonal matrix of the corresponding eigenvalues, $\lambda_{k}$ 's. Each eigenvector $e_{k}$ and its eigenvalue $\lambda_{k}$ are related via the eigenvalue equation, $\mathrm{H} e_{k}=\lambda_{k} e_{k}$, which can also be expressed in terms of the components of the eigenvector as

$$
\int \mathrm{d} \mathbf{x}^{\prime} \mathbf{H}\left(\mathbf{x}, \mathbf{x}^{\prime}\right) e_{k}\left(\mathbf{x}^{\prime}\right)=\lambda_{k} e_{k}(\mathbf{x})
$$

As a result, the harmonic approximation of the potential well provides us with a simple description of the free-energy landscape around the state $m_{0}$. Such a description is given by $\mathbf{D}$ and the corresponding orthogonal set of eigenvectors, $e_{k}$ 's, representing the independent directions of change of $m_{o}$ due to fluctuations of the order parameter, i.e., the normal modes of motion of that configuration.

By expressing the perturbation, $\delta m(\mathbf{x})$, in terms of each normal mode as $\delta \mathrm{m}(\mathbf{x}) \equiv \varepsilon e_{k}(\mathbf{x})$ where $\varepsilon$ is a real number accounting for the magnitude of the perturbation, equation 4.3 can be rewritten as

$$
\begin{aligned}
\Delta \mathcal{F} & \simeq \frac{1}{2} \varepsilon^{2} \int \mathrm{d} \mathbf{x}\left(\int \mathrm{d} \mathbf{x}^{\prime} \mathbf{H}\left(\mathbf{x}, \mathbf{x}^{\prime}\right) e_{\mathrm{k}}\left(\mathbf{x}^{\prime}\right)\right) e_{\mathrm{k}}(\mathbf{x}) \\
& \simeq \frac{1}{2} \varepsilon^{2} \lambda_{\mathrm{k}} \int \mathrm{d} \mathbf{x} e_{\mathrm{k}}(\mathbf{x}) \cdot e_{\mathrm{k}}(\mathbf{x}) \\
\Delta \mathcal{F} & \simeq \frac{1}{2} \varepsilon^{2} \lambda_{\mathrm{k}}\left|e_{\mathrm{k}}\right|^{2} \mathrm{~V}
\end{aligned}
$$

where $V=L_{X} \cdot L_{Y} \cdot L_{Z}$ is the size of the simulation box, and the norm of the eigenvector is defined as

$$
\left|e_{k}\right|^{2}=\frac{1}{V} \int \mathrm{d} \mathbf{x} e_{k}(\mathbf{x}) \cdot e_{k}(\mathbf{x})
$$

In light of this equation, the free-energy change, $\Delta \mathcal{F}$, can be explicitly understood as the result of the motion of the configuration $m_{0}$ along a particular normal mode; in addition, the corresponding eigenvalue, $\lambda_{k}$, describes the freeenergy cost of that motion.

The sign of $\Delta \mathcal{F}$ depends solely on the value of $\lambda_{k}$. When $\lambda_{k}>0$, the potential has a positive curvature in the direction of the $k^{\text {th }}$ eigenvector and the larger the value of $\lambda_{k}$, the larger the free-energy cost associated with a change in the configuration in that direction. When $\lambda_{k}=0, e_{k}$ describes a change with no 
free-energy cost as is the case of translation or rotational modes related to the symmetry of the free-energy functional. For $\lambda_{k}<0$, the potential has a negative curvature in the direction of $e_{k}$, hence, the system is unstable.

Upon computing the free energy of the system, we can proceed to construct the Hessian matrix whose eigenvectors and eigenvalues will provide information about the associated composition fluctuations.

$$
\begin{array}{lll}
\lambda_{k}<0 & \Delta \mathcal{F}<0 & \text { unstable configuration due to mode } k \\
\lambda_{k}=0 & \Delta \mathcal{F}=0 & \text { symmetry-related modes } \\
\lambda_{k}>0 & \Delta \mathcal{F}>0 & \text { configuration unconditionally stable, } \\
& & \text { k is a fluctuation mode }
\end{array}
$$

Considering normalized eigenvectors, $\Delta \mathcal{F}$ can be estimated as

$$
\Delta f=\frac{\Delta \mathcal{F}}{V} \simeq \frac{1}{2} \varepsilon^{2} \lambda_{k}
$$

\subsubsection{Numerical computation of fluctuation modes}

In the case of a block-copolymer system described by a continuum model, the straightforward access to the calculation of the free energy provides a significant advantage for the study of fluctuation modes.

The Hessian coefficients in equation 4.4 are to be computed from the discrete order parameter $m_{i}$, therefore we deploy the numerical approximation of the second-order derivative of the free-energy functional as described in detail in chapter 2 (cf. page 33).

$$
H_{i j} \simeq \frac{1}{\mathbf{\Lambda}}\left(-\varpi+2 \gamma m_{i}+3 \vartheta m_{i}^{2}\right) \delta_{i, j}-\frac{\epsilon}{\mathbf{\Lambda}} \triangle \delta_{i, j}+\alpha G(i, j) \quad(2.73 \text { revisited })
$$

The Hessian matrix obtained from the Ohta-Kawasaki free-energy functional is a symmetric-dense, real matrix; consequently, the spectrum of eigenvalues contains no complex entries. As mentioned earlier, the free-energy change induced by perturbing a given configuration can be estimated via equation 4.3 by using each of the eigenvectors. By comparing this approximation to the exact free-energy change computed from the corresponding order parameter, $\Delta \mathcal{F}=\mathscr{F}[\mathrm{m}]-\mathcal{F}\left[\mathrm{m}_{\mathrm{o}}\right]$, it is possible to test the calculation of the Hessian matrix and of its spectrum and eigenvectors.

As indicated by equation 4.6 , the change in the free-energy density, $\Delta \mathrm{f}=$ $\Delta \mathcal{F} / \mathrm{V}$, linearly increases with $\varepsilon^{2}$ for a given eigenvector. If eigenvectors are 
normalized and $\Delta f$ is rescaled by $\lambda_{k}$, the rate of that linear increase is the same regardless of the eigenvector selected, i.e.,

$$
\frac{\Delta f}{\lambda_{k}} \simeq \frac{1}{2} \varepsilon^{2}
$$

To test this relationship in our numerical calculations, we deploy a lamella phase of a symmetric block copolymer in a 2D system of dimensions $\mathrm{L}_{X}=$ $23.4883 \xi_{0}$ and $L_{Y}=19.4512 \xi_{0}$, where $\xi_{0}$ is the unit of length of the continuum model. The system size in the direction perpendicular to the lamella, $X$, is commensurate to the equilibrium lamellar spacing $L_{O}=11.7441 \xi_{0}$ in order to obtain a stress-free system, $\mathrm{L}_{X} / \mathrm{L}_{\mathrm{O}}=2$. The collocation lattice utilized to compute the minimum of the Ohta-Kawasaki free-energy functional at $\alpha=0.0845$ includes $\mathrm{N}=3392$ cells, where one $\mathrm{L}_{\mathrm{O}}$ corresponds to 32 cells. This configuration is shown in figure 4.1

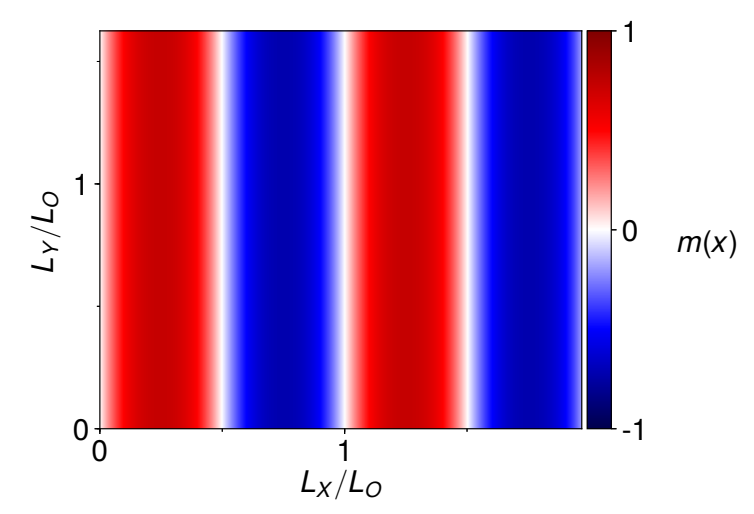

Figure 4.1: Lamella phase of a symmetric block copolymer represented as the order parameter field $m_{0}$. The configuration correspond to the minimum of the Ohta-Kawasaki free-energy functional at $\alpha=0.0845$.

For comparison, figure 4.2 presents the values predicted by equation 4.7 as well as the free-energy change due to the perturbation of the lamella phase $m_{0}(\mathbf{x})$ with the eigenvectors of its corresponding Hessian Matrix for the lowest part of the spectrum of eigenvalues. Eigenvectors $e_{k}(\mathbf{x})$ are normalized and used to compute the modified configurations $m(\mathbf{x})=m_{0}(\mathbf{x})+\varepsilon e_{k}(\mathbf{x})$. The freeenergy change is computed using the Ohta-Kawasaki free-energy functional with the perturbed configurations as $\Delta \mathcal{F}=\mathcal{F}[\mathrm{m}(\mathbf{x})]-\mathcal{F}\left[\mathrm{m}_{\mathrm{o}}(\mathbf{x})\right]$. The free-energy density is computed as $\Delta f=\Delta \mathcal{F} / A$, where $A=L_{X} * L_{Y}=456.876 \xi_{0}^{2}$.

The second-order approximation described by equations 4.2 and 4.3 only holds for small free-energy changes around the equilibrium configuration $\mathrm{m}_{\mathrm{o}}(\mathbf{x})$ 
where $\Delta \mathcal{F}$ can be described as a parabolic function of the magnitude of the perturbation $\varepsilon$. This is the case in our numerical calculations for values of $\varepsilon^{2} \lesssim 0.025$ where regardless of the eigenmode, the free-energy change follows the linear behavior described by equation 4.7 (dashed black line).

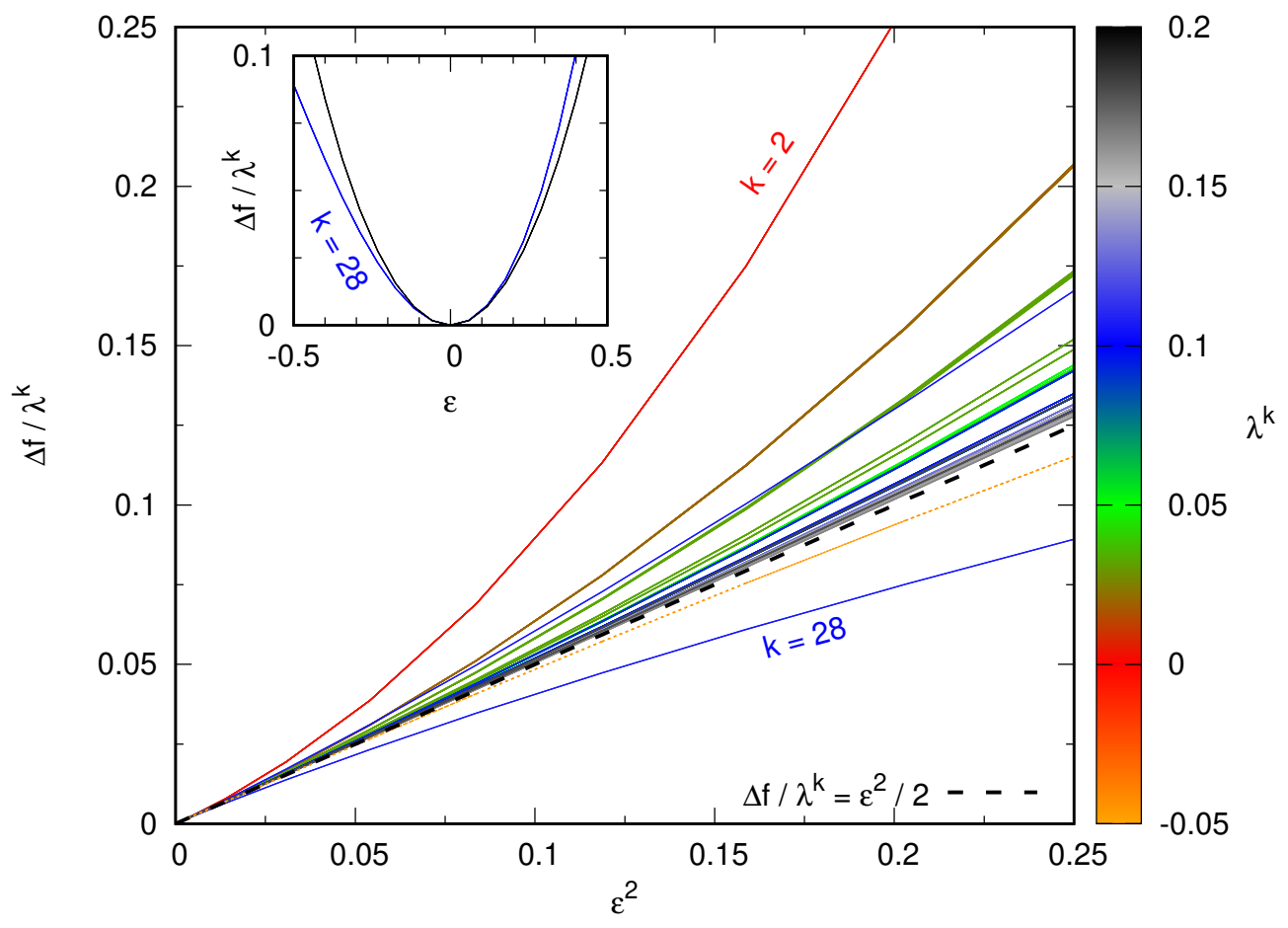

Figure 4.2: The eigenvectors $e_{k}$ of the Hessian matrix of $m_{o}(\mathbf{x})$ are used as a perturbation: $\mathfrak{m}(\mathbf{x})=\mathfrak{m}_{0}(\mathbf{x})+\varepsilon \boldsymbol{e}_{k}(\mathbf{x})$. The plot shows the corresponding freeenergy density change $\Delta f=\left(\mathcal{F}[\mathrm{m}]-\mathcal{F}\left[\mathrm{m}_{\mathrm{o}}\right]\right) / A$ as a function of $\varepsilon^{2}$. The dashed line shows the prediction of the second-order approximation of $\Delta f$.

Beyond $\varepsilon^{2} \gtrsim 0.025$, i.e, $\varepsilon \gtrsim 0.16$, deviations become more important and only some modes follow the predicted linear behavior. These deviations can be expected given the approximation of small free-energy changes around $m_{0}(\mathbf{x})$ and our numerical calculations provide a reference to establish the limits of this second-order approximation.

Two modes deviating from the linear behavior are highlighted in figure 4.2: $k=2$ (red line) and $k=28$ (blue line). The first mode, $k=2$, corresponds to the smallest significant change in the free energy with an associated eigenvalue of $\lambda_{2}=0.0036$. When $\lambda_{k} \rightarrow 0, \Delta f \rightarrow 0$ as expected from equation 4.6; moreover, $\Delta f / \lambda_{k}$ deviates more strikingly due to the rapid increase of higher order terms. 
The later is more clear when one considers the Taylor expansion in terms of $\varepsilon$

$$
\begin{aligned}
\Delta f & \simeq \frac{1}{2} \lambda_{k} \varepsilon^{2}+\frac{1}{3 !} \eta \varepsilon^{3}+\ldots \\
\frac{\Delta f}{\lambda_{k}} & \simeq \frac{1}{2} \varepsilon^{2}+\frac{1}{3 !} \frac{\eta}{\lambda_{k}} \varepsilon^{3}+\ldots
\end{aligned}
$$

where we can observe the third and higher order terms diverge as $\lambda_{k} \rightarrow 0$. Therefore, for mode $k=2$ the ratio $\Delta f / \lambda_{k}$ rapidly increases and deviates from the linear approximation given the small value of $\lambda_{2}$. For the limiting case of $\lambda_{k}=0$, corresponding to the mode $k=1$, in figure 4.3 we can observe the free-energy change due to the fluctuation of this mode is negligible.

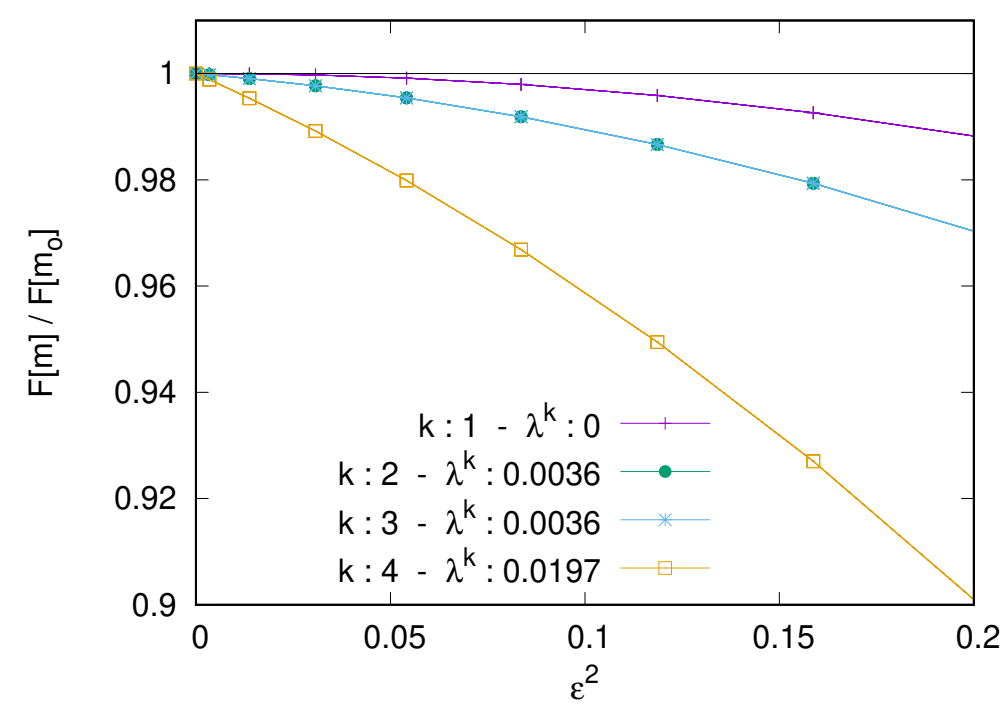

Figure 4.3: Free-energy change for the soft modes corresponding to the first 4 non-negative eigenvalues. The free energy change for mode $k=1$ is negligible whereas, at the limit of the harmonic approximation $\varepsilon^{2} \simeq 0.025$, fluctuations due to mode 4 posses a free energy of about $2 \%$ less than the unperturbed lamella.

For the second mode, $\mathrm{k}=28, \lambda_{28}=0.1059$, the source of the deviation is a different one. As shown in the inset of figure 4.2, the free-energy change corresponding to the perturbation due to this mode does not follow a parabolic form at large values of $\tilde{\varepsilon}$ The asymmetry in the relation, $\Delta f / \lambda_{k}$ vs. $\mathcal{E}$, for this mode implies that higher-order terms of the Taylor expansion (cf. equation 4.2) approximating $\Delta \mathcal{F}$ are required for an accurate description. The latter can also be understood via equation 4.8 , where for both modes deviating from the linear behaviour the ratio $\eta / \lambda_{k}$ in the third order term is large. however, for the 
asymmetric mode $k=28$ that ratio is large due to large values of $\eta$. Thus, the deviation from the linear behavior reveals the mismatch between the freeenergy change computed via the free-energy functional for the block copolymer system and the approximation neglecting the terms of third and higher orders in the Taylor expansion.

One more interesting feature from the results shown in figure 4.2, which has not been analyzed yet in detailed, is the existence of a negative eigenvalue in the spectrum $\lambda_{k}=-0.05$. As previously stated, a negative eigenvalue indicates that the configuration is not (meta)stable. Fluctuations due to the mode corresponding to $\lambda_{k}<0$ would decrease the free energy of the configuration, thus leading towards a more stable state. Because the configuration $m_{0}(\mathbf{x})$ corresponds to a lamellar phase obtained using Cahn-Hilliard dynamics (Model B), we know this represents an equilibrium state at the values of the model parameters used. Therefore, we proceed to analyze this eigenmode more in detail. To start this task, we revisit the requisites and features that the spectrum of eigenvalues and its corresponding eigenvectors must satisfy from the mathematical and physical standpoints for the block-copolymer system of our interest.

As mentioned earlier, the Hessian is a symmetric and dense-real matrix whose eigenvalues must be all real. This condition holds in our numerical calculations, and hence, it is reasonable to expect negative values of $\lambda_{k}$. Nevertheless, no mathematical condition is imposed on the eigenvectors or their corresponding elements, their norm or their properties, other than they should be real vectors of the same dimension as that of the Hessian matrix. Therefore, from the standpoint of the mathematics underlying our calculations, we are left with no conditions to discard the eigenvector corresponding to $\lambda_{k}<0$.

Provided that composition perturbations are constrained by the conserved dynamics we deploy, $\int \mathrm{d} \mathbf{x} \delta \mathrm{m}(\mathbf{x})=0$, the eigenvectors must satisfy $\int \mathrm{d} \mathbf{x} e_{k}(\mathbf{x})=$ 0 ; consequently their average must be zero because of our choice of $\delta \mathrm{m}=$ $\varepsilon e_{k}(\mathbf{x})$. To verify that this condition holds in our numerical results, figure 4.4 shows the average of every eigenvector together with the corresponding spectrum of eigenvalues.

Two modes, however, fail to satisfy the mass conservation constraint. The first of them, $k=0$, corresponds to the mode with a negative eigenvalue observed in the results of figure 4.2. Because this mode is not satisfying neither the stability condition nor mass conservation, perturbations of the equilibrium configuration, $m_{\mathrm{o}}(\mathbf{x})$, possess no physical meaning and should not be included in the analysis. Nevertheless, the second mode that violates mass conservation, $k=46$ and $\sum_{i}\left\{e_{i}^{k}\right\}=4460.0$, belongs to the positive part of the spectrum.

At this juncture, it is useful to express the free-energy change in terms of the 


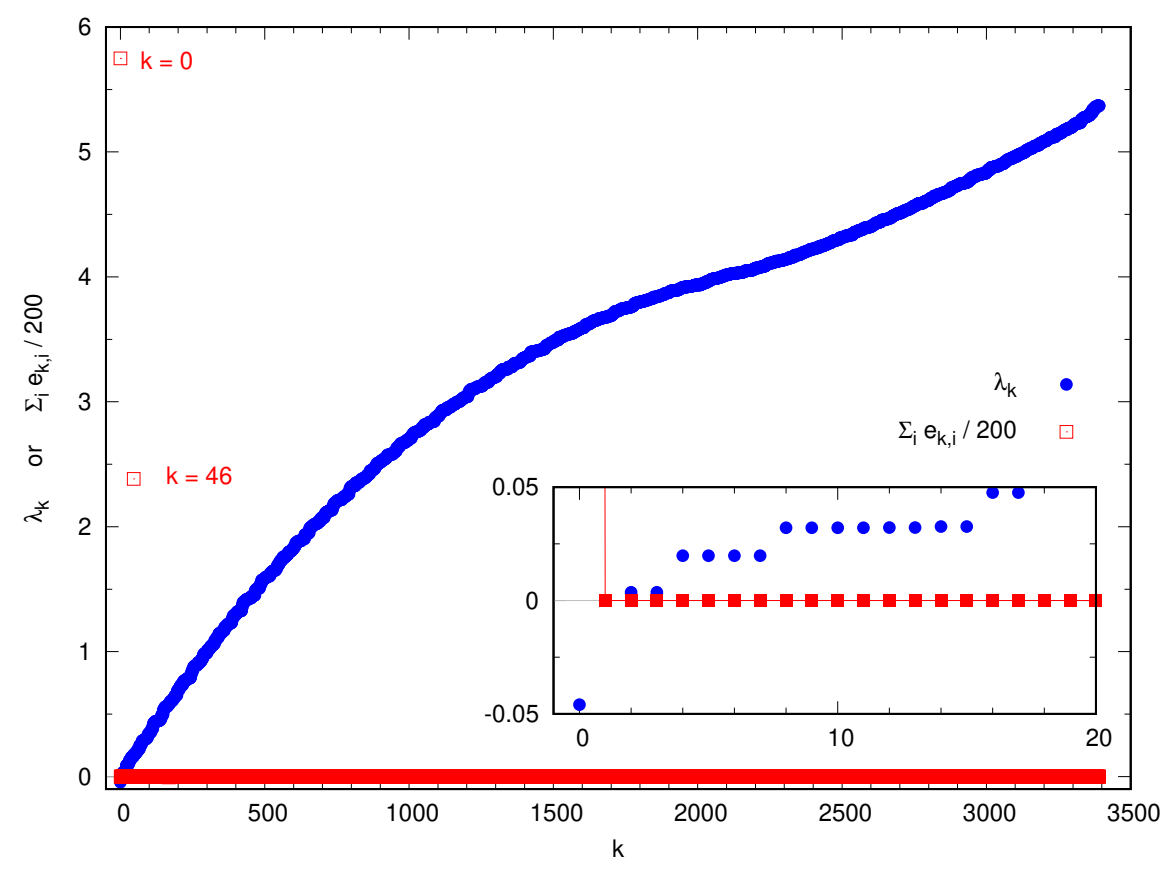

Figure 4.4: Average of every eigenvector (red) and the corresponding eigenvalues (blue) of the Hessian matrix $H$. The average is rescaled as $\sum_{i}\left\{e_{k, i}\right\} / 200$ to facilitate visualization.

Hessian matrix $\mathbf{H}$ by resorting to the matrix form of equation 4.5 given below

$$
\Delta \mathcal{F} \simeq \frac{1}{2} e_{k}^{\top} H e_{k}
$$

In view of the mass-conservation constraint, we know that $\Delta \mathcal{F}$ must be expressed in terms of a $(\mathrm{N}-1)$ vector sub-space where $\mathrm{N}$ is the dimension of $\mathbf{H}$. This reduction by one dimension constitutes the mathematical expression of the physical constraint that the system must satisfy. Therefore, the $\mathrm{N}$-dimensional space containing the current representation of $\mathrm{H}$ must be transformed into a space with one known vector and $(\mathrm{N}-1)$ unknown vectors with zero-average, thus satisfying mass conservation. One trivial educated guess for that known vector is a uniform change of the composition $\delta \mathrm{m}(\mathbf{x})=\mathrm{c}$. Clearly, this constant vector has a non-zero average of $\sum_{i}^{N} e_{i}^{k} / N=c N / N=c$.

For all the other $(\mathrm{N}-1)$ vectors to satisfy mass conservation, a transformation must be performed. Such a transformation is defined in terms of the difference between each vector and its average; by definition, the resulting vector 
satisfies mass conservation. The proposed transformation can be expressed as:

$$
\begin{aligned}
\tilde{e}_{k} & =e_{k}-\frac{1}{N} e_{k} \mathbf{1} \\
& =\mathbf{P} e_{k}
\end{aligned}
$$

where $\mathrm{N}$ is the dimension of the eigenvector $e_{k}$ and $\mathbf{1}$ is the unit $\mathrm{N} \times \mathrm{N}$ matrix; moreover, the linear operator $\mathbf{P}$ is defined as

$$
\mathbf{P}=\left(\mathrm{I}-\frac{1}{\mathrm{~N}} \mathbf{1}\right)
$$

where $\mathbf{I}$ is the identity matrix, thus indicating that $\mathbf{P}$ is a symmetric, real matrix.

Matrix $\mathbf{P}$ acts as a projection of the vectors $e_{k}$ onto the subspace of vectors with zero average. Thus, this linear operator must fulfill the properties of a projector matrix: $\mathbf{P}^{\top}=\mathbf{P}$ and $\mathbf{P}^{2}=\mathbf{P}$. The first condition should be clear because $\mathbf{P}$ is symmetric. Herein, it is useful to recall that $\mathbf{1}^{2}=\mathrm{N} \mathbf{1}$ where $\mathbf{1}$ is a $\mathrm{N}$ $x \mathrm{~N}$ matrix. Thus, we can prove the second condition holds so $\mathrm{P}$ is indeed a projector:

$$
\begin{aligned}
\mathbf{P}^{2} & =\left(\mathrm{I}-\frac{1}{\mathrm{~N}} \mathbf{1}\right)\left(\mathrm{I}-\frac{1}{\mathrm{~N}} \mathbf{1}\right) \\
& =\left(\mathrm{I}-\frac{1}{\mathrm{~N}} \mathbf{1}\right) \mathrm{I}-\left(\mathrm{I}-\frac{1}{\mathrm{~N}} \mathbf{1}\right) \frac{1}{\mathrm{~N}} \mathbf{1} \\
& =\mathrm{I}-\frac{1}{\mathrm{~N}} \mathbf{1}-\frac{1}{\mathrm{~N}} \mathbf{1}+\frac{1}{\mathrm{~N}^{2}} \mathbf{1}^{2} \\
& =\mathrm{I}-\frac{1}{\mathrm{~N}} \mathbf{1}-\frac{1}{\mathrm{~N}} \mathbf{1}+\frac{1}{\mathrm{~N}} \mathbf{1} \\
& =\left(\mathrm{I}-\frac{1}{\mathrm{~N}} \mathbf{1}\right)=\mathrm{P}
\end{aligned}
$$

As a result, the approximation for $\Delta \mathcal{F}$ in equation 4.9 can only be valid once the vector space of the Hessian matrix $\mathrm{H}$ is projected using $\mathbf{P}$ in order to satisfy the constraint:

$$
\begin{aligned}
& \Delta \mathcal{F} \simeq \frac{1}{2}\left(e_{k} \mathbf{P}\right)^{\top} \mathbf{H}\left(\mathbf{P} e_{k}\right) \\
& \simeq \frac{1}{2} v_{\mathrm{k}} \mathbf{G} v_{\mathrm{k}}
\end{aligned}
$$

where $\mathbf{G}=\left(\mathbf{P}^{\boldsymbol{T}} \mathbf{H} \mathbf{P}\right)$ is a transformed Hessian matrix and $v_{\mathrm{k}}$ denotes its corresponding eigenvectors with eigenvalues $\lambda_{k}^{G}$. 
For all the vectors satisfying $P e_{k}=e_{k}$, i.e., the sum over the entries of $e_{k}$ is zero, the free-energy change can be expressed by using the Hessian matrix, with or without the transformation, as follows:

$$
\Delta \mathcal{F} \simeq \frac{1}{2} e_{k} H e_{k}=\frac{1}{2} v_{k} \mathbf{G} v_{k}
$$

therefore $v_{k}=e_{k}$ and for this subset of vectors, we have

$$
\begin{aligned}
\mathbf{G} v_{k} & =\lambda_{k}^{G} v_{k} \\
& =\left(\mathbf{P}^{\mathbf{T}} \mathbf{H} \mathbf{P}\right) v_{k} \\
& =\mathbf{P}^{\mathbf{T}} \mathbf{H} v_{k} \\
& =\lambda_{k}^{\mathrm{H}}\left(\mathbf{P} v_{k}\right) \\
& =\lambda_{k}^{\mathrm{H}} v_{k} \\
\lambda_{k}^{G} & =\lambda_{k}^{\mathrm{H}}
\end{aligned}
$$

Moreover, we have suggested that the constant vector, $\delta \mathrm{m}(\mathbf{x})=\mathrm{c}$, should be an eigenvector of $\mathbf{G}$; to prove this, we select $v_{k}=\vec{c}=\overrightarrow{1}$. For this particular vector, $\mathbf{P} v_{k}=0$ leading to $\mathbf{G} v_{k}=0$. The value of $\lambda_{k}$ for which the eigenvector equation holds with this vector is $\lambda_{k}=0$. Therefore, a constant vector $v_{k}=\vec{c}$ is an eigenvector of $\mathbf{G}$ with a corresponding eigenvalue of $\lambda_{k}=0$.

We can conclude now that the transformed Hessian matrix $\mathbf{G}$ is the projection of $\mathbf{H}$ onto the $\mathrm{N}-1$ subspace of vectors with zero average fulfilling mass conservation. That subspace, is the orthogonal complement to the 1D subspace spanned by $\overrightarrow{\mathbf{1}}$ where the vector defined by the physical constraint of our system belongs, which has a non-zero average and a zero eigenvalue. Consequently, we use $\mathbf{G}$ instead of $\mathbf{H}$ for the calculation of the fluctuation modes.

Figure 4.5 shows the spectrum of eigenvalues and the average of the eigenvectors of $\mathbf{G}$ for the same lamella system previously considered. As expected, the average of all the eigenvectors is zero except for $k=1$. This eigenvector corresponds to the one with a predicted constant value.

Regarding the eigenvalues, all the values are non-negative which fulfill the stability condition of a positive spectrum, i.e. a positive Hessian matrix. Modes with a zero eigenvalue do not violate the stability condition. As expected for those eigenvectors of $\mathbf{H}$ with zero average, i.e., the majority of vectors as shown in figure 4.5, the spectrum of eigenvalues of the matrix $\mathbf{H}$ agrees with that of $\mathbf{G}$ because we proved that $\lambda_{k}^{G}=\lambda_{k}^{H}$ for that set of eigenvectors. 


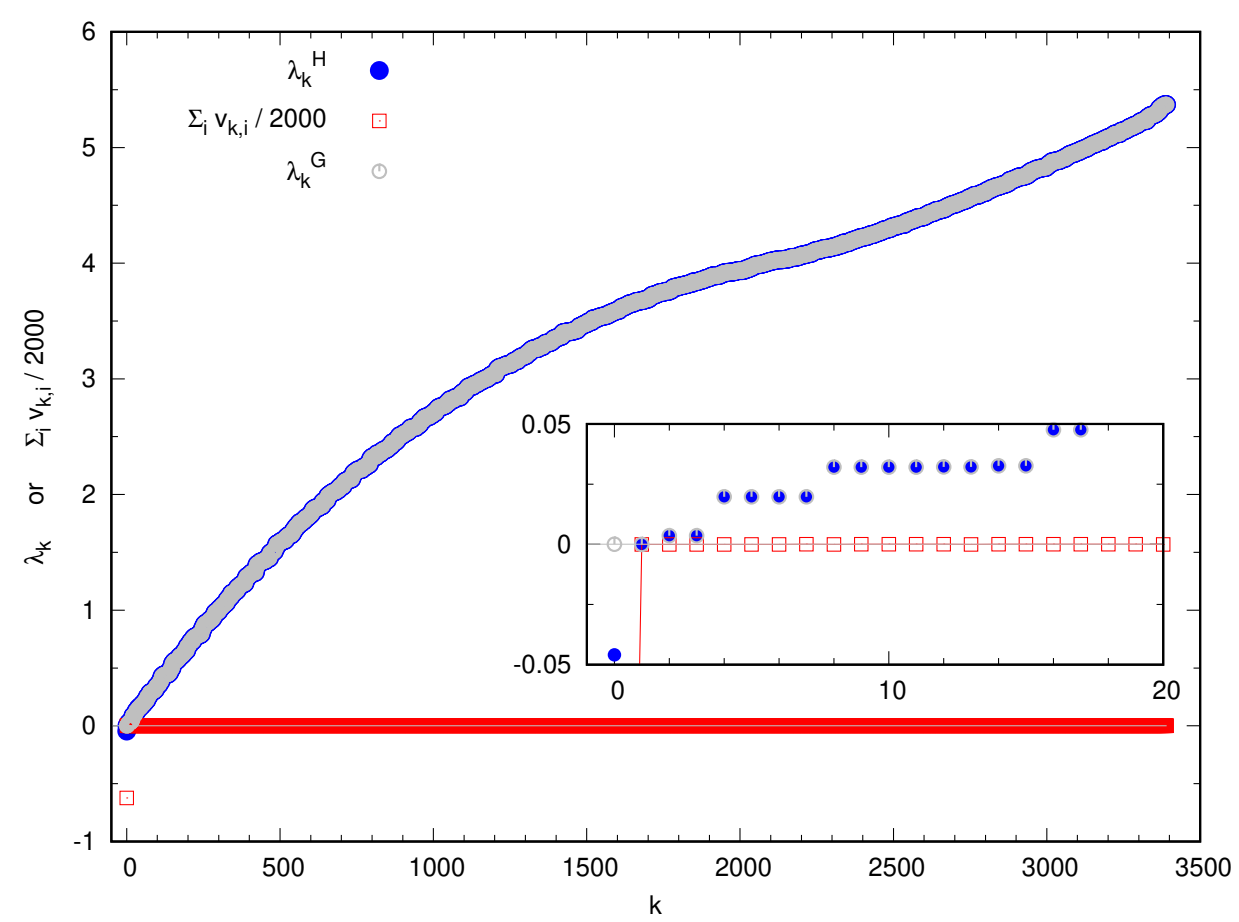

Figure 4.5: Average of every eigenvector (red) and the corresponding eigenvalues (gray) of the transformed Hessian matrix G. The eigenvector average is rescaled as $\sum_{i}\left\{v_{i}^{k}\right\} / 2000$ to facilitate visualization.

\subsubsection{Fluctuation modes of defect-free structures}

With a valid set of eigenvectors representing the local changes of the order parameter, it is possible to describe the local fluctuations associated with every eigenvector of that space.

It has been previously shown that fluctuations associated to modes with $\lambda_{k}=0$ do not change the free-energy of the corresponding stable state, which is the case for modes $k=0$ and $k=1$ (see figure 4.6). Mode $k=0$ is discarded from the analysis since the associated fluctuations violate mass conservation.

To understand the nature of mode $k=1$, it is useful to recall that fluctuations where $\Delta \mathcal{F}=0$ are related to the continuous symmetries of the free-energy functional. The Ohta-Kawasaki model is translational and rotational invariant; however, the translation, $\mathscr{F}[m(\mathbf{x})]=\mathscr{F}[\mathrm{m}(\mathbf{x}+\varepsilon)]$, is the only continuous symmetry. For a given system size, only certain rotations of $m(x)$ leave the free energy unchanged. That is the case for 90-degree rotations, and multiples of it, in a square $2 \mathrm{D}$ system commensurate to the equilibrium spacing $\mathrm{L}_{\mathrm{O}}$, i.e., a system with $L_{Y}=L_{X}=n L_{O}$. Therefore rotation is not a continuous symmetry of the 

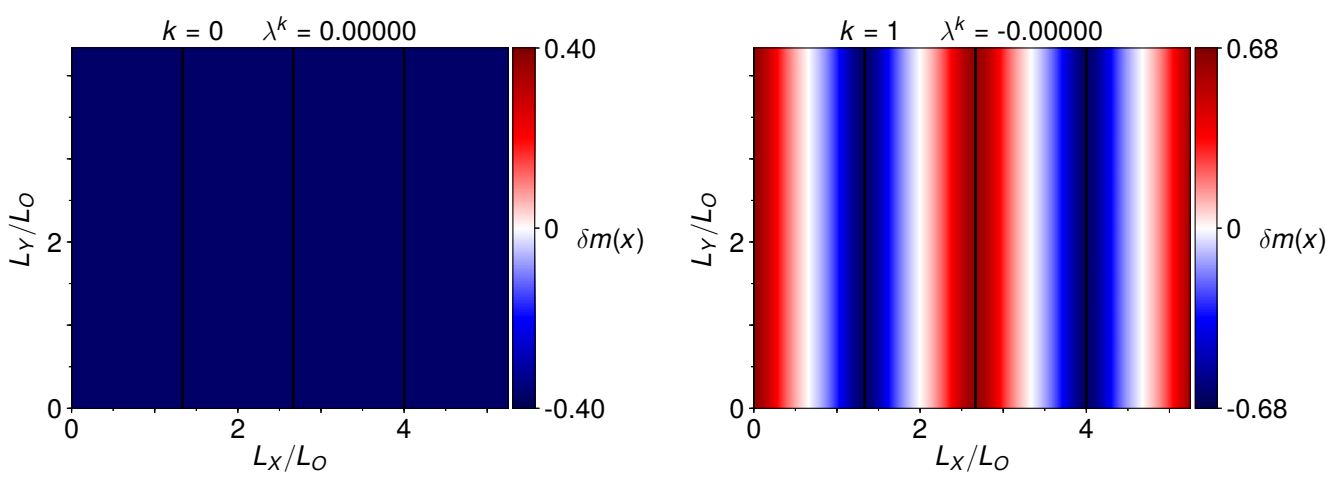

Figure 4.6: First two eigenvectors (color map) of the transformed Hessian matrix $\mathbf{G}$ associated with no change in the free energy during a perturbation. The mode $k=0$ corresponds to constant change of the local composition and fails to satisfy mass conservation. Therefore, changes in the configuration due to this mode provide no physical insights. Black lines signify the AB interfaces of $m_{0}$.

free-energy functional.

Provided that a fluctuation mode corresponding to the translational symmetry exists, the following expression must be satisfied.

$$
\begin{aligned}
\mathrm{m}(\mathbf{x}+\varepsilon) & =\mathrm{m}(\mathbf{x})+\varepsilon e_{k}(\mathbf{x}) \\
e_{k}(\mathbf{x}) & =\frac{\mathrm{m}(\mathbf{x}+\varepsilon)-\mathrm{m}(\mathbf{x})}{\varepsilon}
\end{aligned}
$$

This expression corresponds to a simple numerical approximation for the firstorder derivative, $\mathrm{dm} / \mathrm{dx}$. Figure 4.7 confirms that mode $\mathrm{k}=1$ corresponds to the first-order derivative of the lamella configuration $m_{o}$ by comparing their profiles along the $X$ direction. Moreover, provided that translation is a continuous symmetry of this model, the eigenvalue of such mode must be zero. This is the case for mode $k=1$ and therefore this eigenvector corresponds to the translation mode of the lamella configuration.

We now proceed to investigate other fluctuation modes that can be expected due to broken symmetries. The formation of ordered phases of block-copolymers from the disordered state resembles the crystallization process observed in hard crystals. This trait has been exploited to draw parallels and investigate these soft matter systems building on the extensive knowledge of atomic crystals [102]. An important difference, however, must be highlighted: in self-assembled phases of block copolymers, it is the collective density what undergoes a crystallizationtype ordering and not the coordinates of individual particles ${ }^{\mathrm{i}}$. This is evident

\footnotetext{
${ }^{\mathrm{i}}$ We refer to particles recalling that the collective density description in a continuum model correspond to the underlying particle based description of a block copolymer chain.
} 


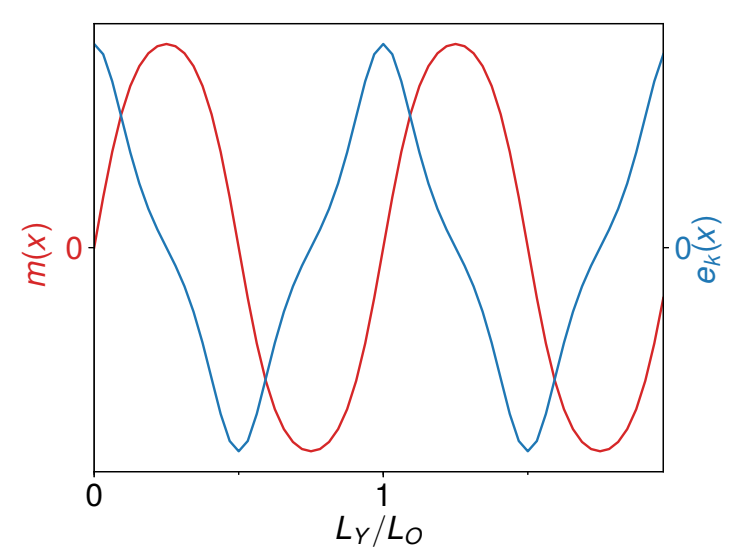

Figure 4.7: Comparison of the composition profile along $X$ for the lamella configuration (red line), shown in figure 4.1, with the eigenvector profile corresponding to the mode $k=1$ (blue line), shown in figure 4.6. The profile of the eigenvector, $e_{k}(\mathbf{x})$, corresponds to the first derivative of the composition profile, $\mathrm{m}(\mathbf{x})$, thus confirming that this vector describes a translation mode.

in the choice of the order parameter: for the soft matter system of our interest, the order parameter is the local densities of the A or B blocks of the polymer chains, whereas in crystals it is the local displacements of atoms from their lowest-energy lattice positions. Nevertheless, a useful parallel can be drawn between the fluctuation modes of our system (i.e., the eigenvectors of the Hessian matrix of the free energy of the block-copolymer system) and the phonons (i.e., the eigenvectors of the potential energy) representing the elementary energetic fluctuations in crystals.

Phonons and the broken symmetries in crystals are related via the Goldstone theorem; due to the spontaneous breaking of the continuous translational symmetry, the system exhibits low-energy excitations which have low-frequencies, i.e., Goldstone modes [103, 104]. Thus, the low-frequency phonons in crystals correspond to the Goldstone modes for the translations; whose associated energy cost vanishes in the limit of infinite wavelength [105, 106]

The free-energy cost associated to the deformation of the AB interfaces, $\Delta \mathcal{F}$, has been previously described for the case of small-amplitude undulations as [107]

$$
\Delta \mathcal{F}=F-F_{o} \simeq \int \mathrm{d} \mathbf{r}\left\{\frac{\gamma}{2}[\nabla \delta x(y)]^{2}+\frac{K}{2}[\Delta \delta x(y)]^{2}\right\}+\int \mathrm{d} \mathbf{r} \frac{B}{2}[\delta x(y)]^{2}
$$

where $F_{o}$ corresponds to the free energy of the lamella without deformation, $\delta x(y)$ is the amplitude of the undulation along the interface, and $K$ and $B$ are the bending and compression moduli, respectively. The first term on the right-hand 
side of the above equation accounts for the energy of the capillary waves where the lamellar structure undergoes a collective bending, i.e., the undulation waves are in-phase. In Fourier space, this first term has the form, $\Delta \mathcal{F} \sim \gamma q_{Y}^{2}+\mathrm{Kq}_{Y}^{4}$, where $\mathrm{q}_{\gamma}$ is the wave vector in the direction parallel to the interfaces. When the undulation waves are out of phase, the domains will be compressed or stretched and the corresponding free-energy cost will be described by the second term on the right-hand side of Eq. 4.16.

In this regard, we investigate the variations of the lowest energy fluctuation modes due to changes in the wave vector, $\mathrm{q}_{Y}(\mathrm{k})=2 \pi \mathrm{k} / \mathrm{L}_{\gamma}$. The smallest nonzero eigenvalues $\lambda_{k}$ in the spectrum correspond to the modes with the lowest frequencies, i.e., the longest wavelength; therefore, we investigate modes $2 \leqslant$ $k \leqslant 7$ (cf. figure 4.5). These modes are degenerate such that we express the changes of $\lambda_{k}$ as a function of $L_{Y}$ only for modes $k=2,4,7$.

Figure 4.8 confirms that as the wave vector $q_{Y}(k)$ tends to zero, i.e., $L_{Y}$ tends to infinity, the frequency of modes $k=2,4$ approaches zero as well and their excess free energy vanishes (cf. eq. 4.6). In that limit, these two fluctuation modes approach the translation mode of the configuration, which has an excess free energy of zero as previously discussed. Thus, modes $k=2,4$ are the Goldstone excitations of the lamella due to the spontaneous breaking of the translational symmetry. In contrast, the peristaltic fluctuation due to mode $k=6$ requires the compression of the lamellar phase with an associated excess free energy $\Delta \mathcal{F} \geqslant 0$ regardless of the wave vector $\mathrm{q}_{Y}(\mathrm{k})$.

Furthermore, by resorting to the capillary wave theory of interfaces [108], these Goldstone modes identified in the continuum description of the blockcopolymer system of our work, $k=2,4$, correspond to the thermally excited capillary waves that strongly influence the interface width in block copolymer systems [105, 109, 110].

Fluctuations of the configurations corresponding to a given mode are better described by computing directly the perturbed configurations $\mathbf{m}(\mathbf{x})=\mathfrak{m}_{0}(\mathbf{x})+$ $\varepsilon e_{k}(\mathbf{x})$ where $\varepsilon$ can take any real value. In figure 4.9 , therefore, we consider fluctuations of the same magnitude but opposite sign to show snapshots of the perturbation of the lamella phase with four different modes.

We corroborate that mode $k=0$ corresponds to the translation of the configuration in the direction perpendicular to the lamella, which is natural in view of the symmetry of the system. It is clear now that no free-energy change is associated with this fluctuation. Furthermore, the soft mode $k=2$ corresponds to an undulation of the $\mathrm{AB}$ interfaces and the same type of fluctuation is observed with the corresponding degenerate mode $k=3$, but the undulation wave has a different phase in this case. These three first normal modes correspond to fluctuations arising from the collective motion of the lamella. 
(a)
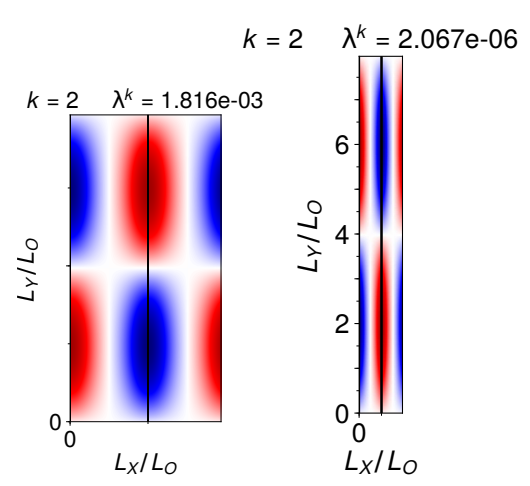

(b)

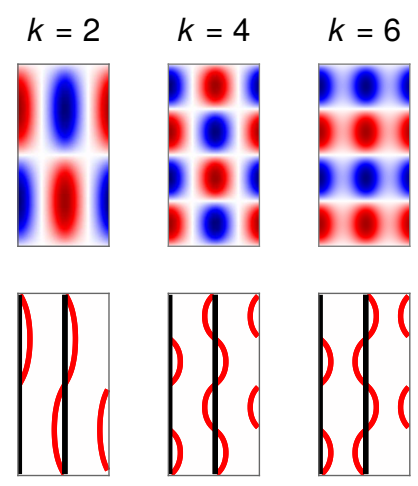

(c)

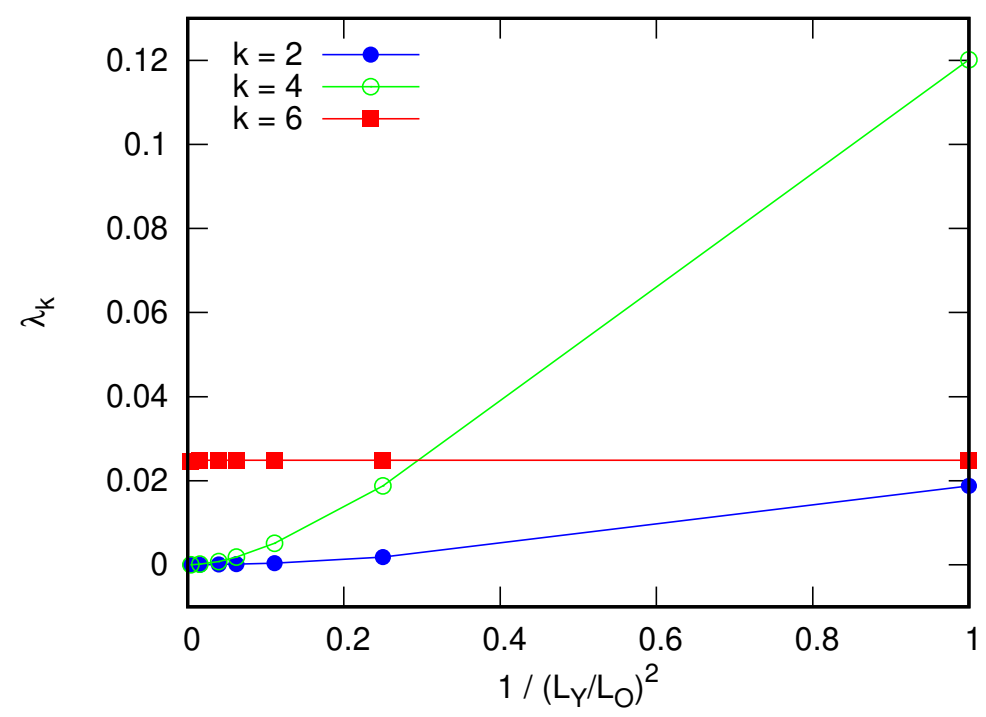

Figure 4.8: Goldstone modes in lamellar phase $m_{0}$. (a) The wavelength of the undulation increases as the wave vector, $\mathrm{q}_{\gamma}(\mathrm{k})=2 \pi \mathrm{k} / \mathrm{L}_{\gamma}$, decays. (b) Lowenergy fluctuation modes (color map) corresponding to the lowest non-zero eigenvalues. The deformation of the $\mathrm{AB}$ interfaces (red contour line) arising from the perturbation of the lamellar phase in equilibrium (black contour line) allows to differentiate the peristaltic motion from the lamellar undulations. (c) The frequency of the peristaltic motion remains constant regardless of wave vector $q_{Y}(k)$. In contrast, the frequency vanishes as $q_{Y}(k) \rightarrow 0$ for the low-line undulations, $k=2,4$. The later indicate that these modes correspond to the Goldstone excitations originated by the broken translational symmetry. 
(a)

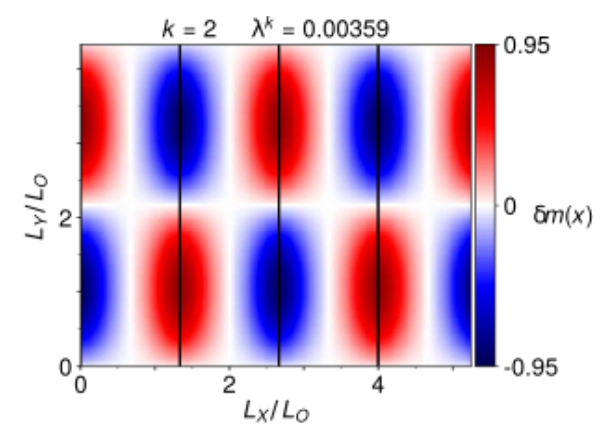

(b) $e^{k}(\mathbf{x})$
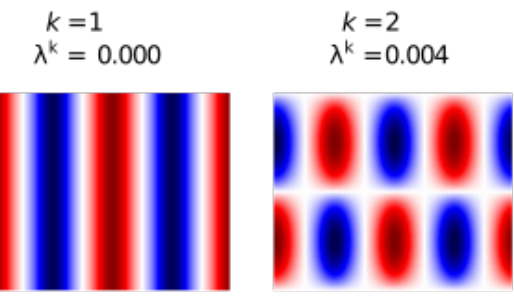

$\begin{aligned} k & =3 \\ \lambda^{k} & =0.004\end{aligned}$

$k=3000$

$\lambda^{k}=4.842$
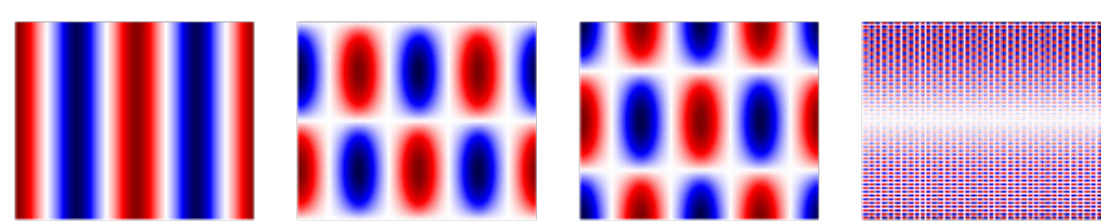

(c) $\mathrm{m}(\mathbf{x})=\mathrm{m}_{0}+\varepsilon e^{\mathrm{k}}(\mathbf{x})$

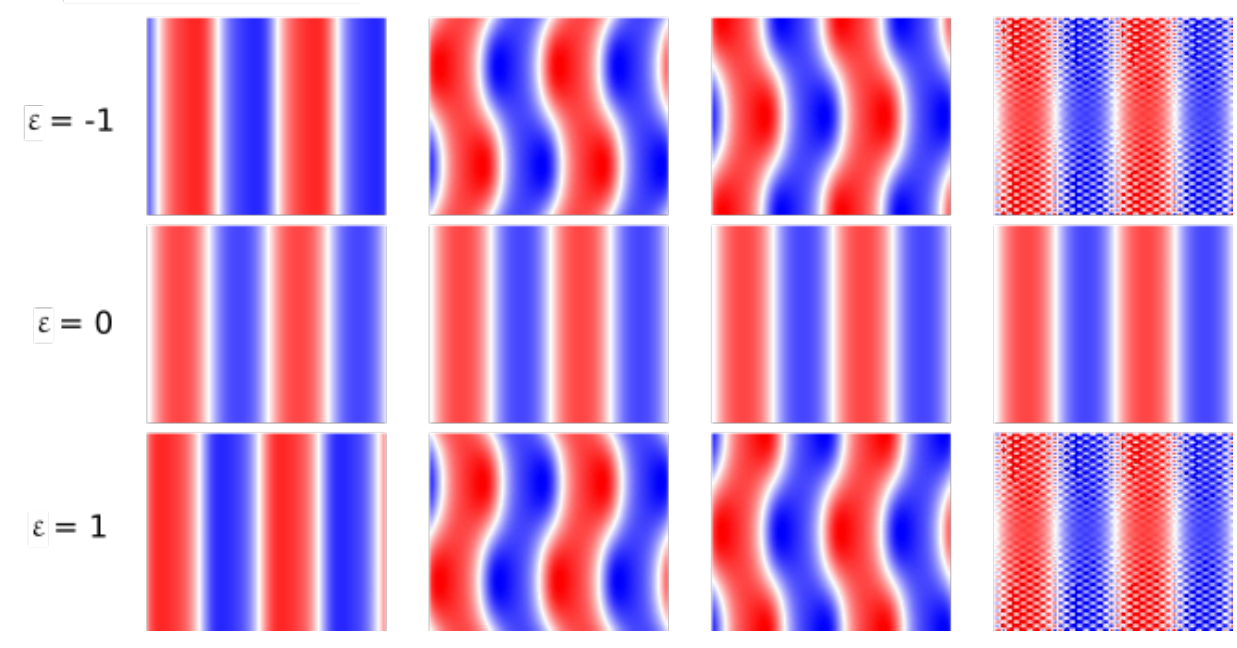

Figure 4.9: Fluctuations of the lamellar phase due to four different modes. (a) A fluctuation mode describes the changes in the local composition, $\delta \mathrm{m}(\mathbf{x})$, which in the case of a soft mode (color map) will cause fluctuations of the AB interfaces of the corresponding unperturbed lamella $m_{\mathrm{o}}$ (black contour lines). (b) Mode $k=1$ depicts changes associated with the translation of the configuration $m_{0}$. Modes $k=2,3$ are degenerate soft modes associated to the lowest free-energy change. Mode $k=3000$, at the last part of the spectrum of eigenvalues, depicts high-frequency local fluctuations. (c) Perturbations of the same magnitude but opposite sign, where white represents the $A B$ interfaces. A value of $|\varepsilon|=1$ is used for better visualization. 
In contrast, as the eigenvalue increases and thus the spatial frequency of the undulations increases the fluctuations correspond to more localized changes. Mode $k=3000$ corresponds to a much higher fluctuation, close to the end of the spectrum, where undulations do not correspond to a displacement of internal $\mathrm{AB}$ interfaces but rather to local composition fluctuations inside the domains.

It is worth pointing out that in the course of the transition of the lamella configuration $m_{0}$ towards another given stable state, the key changes will occur on the AB interfaces. Therefore, we focus our attention on the behavior of such interfaces to investigate the various fluctuation modes.

The lowest fluctuation modes in our study, e.g., $k=9,13,15$, correspond to capillary waves in 2D as depicted in red contour lines in figure 4.10. Despite of having rather close eigenvalues, i.e., a similar wavelength of the undulations, the coupling of the fluctuation of each $\mathrm{AB}$ interface in the lamellar phase is different in every mode, thus indicating that they are not degenerate. The smallest difference between their values suggests that two given eigenvalues, $\lambda_{i}$ and $\lambda_{j}$ will be degenerate if $\Delta \lambda \leqslant 4 \times 10^{-5}$ for the collocation grid deployed in these calculations.

In this regard, the fluctuation modes offer a complementary description of the $\mathrm{AB}$ interfaces in the continuum model, provided that such modes agree with the capillary wave description of interfaces and satisfy the constraints imposed by the symmetry of the free energy functional: the existence of modes describing Goldstone-type excitations and a mode related to the translation of the configuration.

\subsubsection{Fluctuation modes of topological defects}

Hitherto, we have described the physical insights derived from the fluctuation modes in an ordered phase of a block-copolymer system, and we have highlighted the changes that $\mathrm{AB}$ interfaces undergo. In the ordering process of such structures, topological defects are formed and their presence in the structure gives rise to a different class of fluctuations.

The analytical description of various topological defects poses a significant challenge that can be conveniently circumvented via numerical simulation of such configurations in real space. This contrasts with the study of the fluctuation modes of defect-free structures, which can be performed analytically in reciprocal space exploiting the symmetries of these ordered phases.

In this regard, we consider a prototypical dislocation defect in a lamellar phase to study the modes of a defective configuration. We deploy the metastable defect in a lamellar phase of a symmetric block copolymer in a 2D system of dimensions $\mathrm{L}_{X} / \mathrm{L}_{\mathrm{O}}=13$ and $\mathrm{L}_{Y} / \mathrm{L}_{\mathrm{O}}=9.5$ where the equilibrium lamellar spac- 


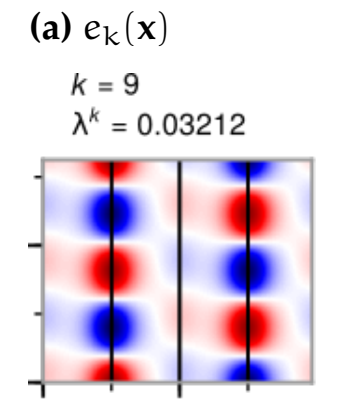

(b) $\mathfrak{m}(\mathbf{x})=\mathrm{m}_{\mathrm{o}}+\varepsilon e_{\mathrm{k}}(\mathbf{x})$ with $\varepsilon=1$

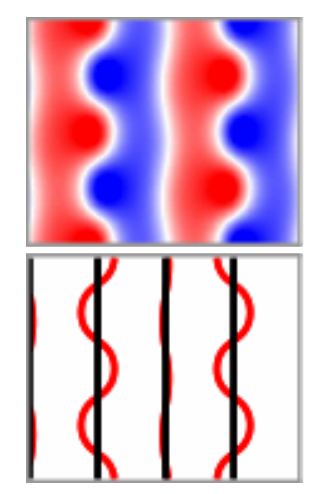

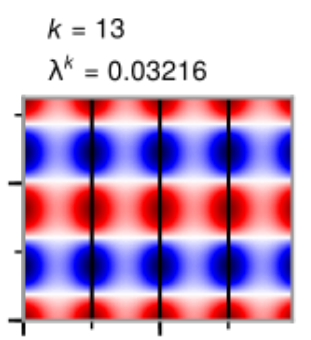

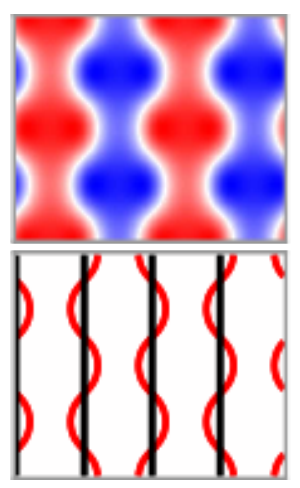

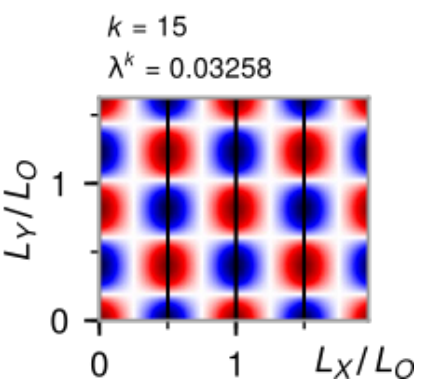

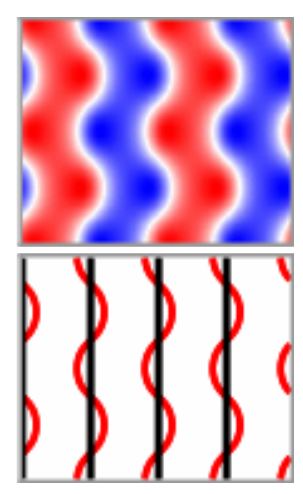

Figure 4.10: Undulations of the $A B$ interfaces due to modes with higher frequencies. (a) Three modes with close values of $\lambda$ describe changes in $m(\mathbf{x})$ with similar periodicity, $\mathrm{L}_{\mathrm{O}}$, but different symmetries. (b) Fluctuations of the AB interfaces of the lamellar phase (black lines) due to the modes depicted in (a). The three modes produce different fluctuation patterns, but the undulation waves (red lines) have almost the same wavelength. A high value of $\varepsilon$ above the limit of the harmonic approximation is used for a better visualization of fluctuations.

ing, $\mathrm{L}_{\mathrm{O}}$, is equal to $13.775 \xi_{0}$. The collocation lattice utilized to compute the minimum of the Ohta-Kawasaki free-energy functional at $\alpha=0.04638$ includes $\mathrm{N}=17,784$ cells where $\mathrm{L}_{O}$ corresponds to 12 cells. This configuration is shown in figure 4.11

Among all the defect fluctuation modes, the subset that mainly alters the local composition around the defect, i.e., local modes, is of particular interest. Figure 4.12 shows how these two types of fluctuation modes alter the AB interfaces of the structure. Changes in the $\mathrm{AB}$ interfaces of the defect and the neighboring lamellae can lead to the merging of the interfaces of the defect cores and further defect annihilation. In addition, as shown with modes $k=2,8$, fluctuations of the defect cores and interfaces of the neighboring lamellae give rise to changes in the long-range strain field created by the presence of the defect in the 


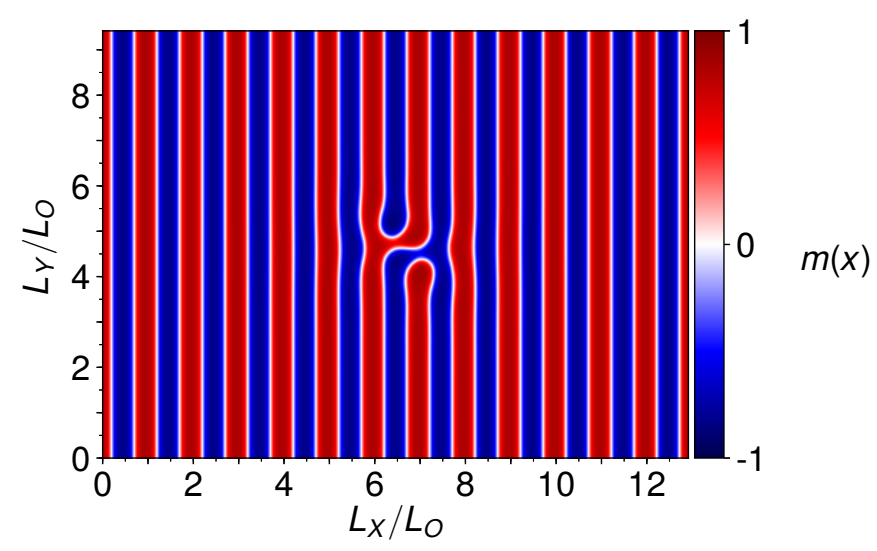

Figure 4.11: Dislocation pair with defect cores of opposed A and B domains.

lamellar phase. The later strain field gives rise to the Peach-Koeler force acting on the pair of dislocations, which are non-local and decay as $\sim 1 / \mathrm{L}$ with $\mathrm{L}$ being the distance between the defect cores $[59,86,111]$.

\subsection{Fluctuation modes and minimum free-energy paths (MFEP)}

As we have shown, fluctuations of a configuration around a local minimum, $\mathrm{m}_{\mathrm{o}}$, can be described by means of the fluctuation modes derived from a harmonic approximation of the corresponding free-energy basin. If there is another basin in the free-energy landscape, e.g., $\mathcal{F}\left[\mathrm{m}_{0}^{*}\right]<\mathcal{F}\left[\mathrm{m}_{0}\right]$, the most probable transition pathway between the two corresponding states is described by the minimum free-energy path (MFEP), which is a family of configurations along which the thermodynamic force is everywhere parallel to the path $[112,113]$. That set of configurations is described as $\mathfrak{m}(\mathbf{x}, \mathbf{s})$ where $s \in[0,1]$ denotes the contour length along the path and $m(x, s=0)=m_{o}$ and $m(x, s=1)=m_{o}^{*}[113,114]$.

There are several methods to find the minimum free-energy path and among them the improved string method provides a simple, stable and accurate procedure [112]. The original string method [115] employs the projection of the potential force onto the direction normal to the path as the actual force of the calculation. This requires the calculation of the tangent vector along the path in order to project the potential force and find the perpendicular component of the potential. This step is eliminated in the improved string method, where the full force is used in the iterative procedure to find $m(x, s)$ [112].

The MFEP allows us to compute the free-energy barriers along the path as 

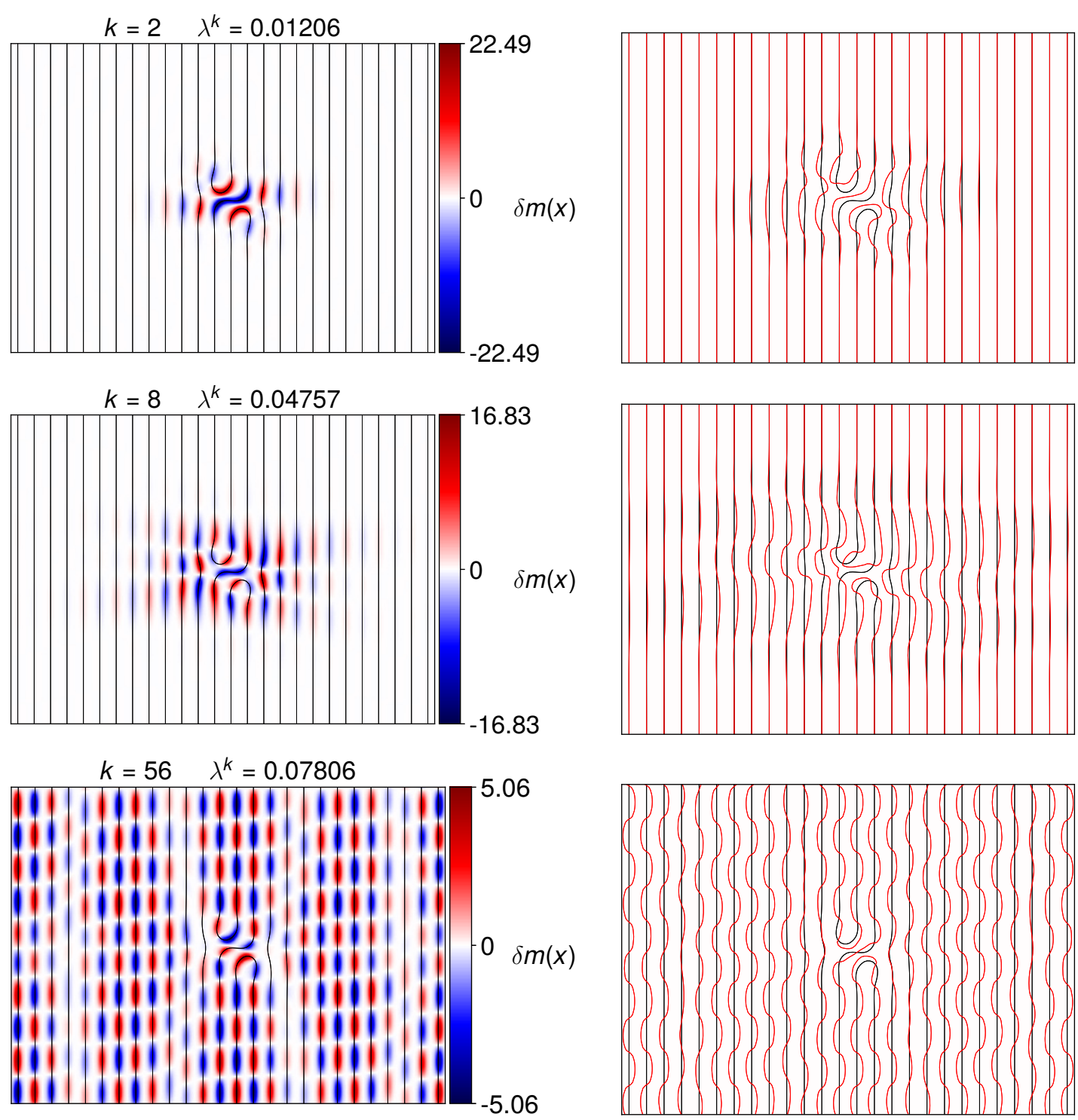

Figure 4.12: Fluctuation modes of the defective configuration can be classified into local and non-local composition changes with respect to the defect. Local modes, e.g., $\mathrm{k}=2,8$, promote changes of $\mathrm{AB}$ interfaces localized around the defect cores as well as the neighboring $A B$ interfaces enhancing the strain field imposed by the defect on the lamellar structure. Non-local modes, e.g., $k=56$, give rise to changes across the entire structure. Color maps describe local composition changes due to each fluctuation mode, black contour lines represent the $A B$ interfaces of the mean-field defect configuration, $m_{0}$, and red contour lines depict the fluctuating defect due to the mode $\mathrm{k}$ with $\varepsilon=1$, i.e., $m(\mathbf{x})=m_{\mathrm{o}}+e_{k}(\mathbf{x})$. 
well as the changes the morphology undergoes in the corresponding transition. In the limit of a vanishing small free-energy barrier, the string method and the fluctuation mode analysis provide the same description of the changes in local composition that the initial configuration, $m(x, s=0)=m_{0}$, undergoes at the onset of the corresponding transition.

In the present section we exploit the latter fact and compare the transition pathway of a defective configuration towards the defect-free lamella with the fluctuation mode analysis of the defect. Such comparison is performed at the limit of meta-stability when the free-energy barrier approaches zero.

\subsubsection{String Method and MFEP}

Given the continuum description of the system in our studies, the configurations along the string are represented by the order parameter field $m(x)$ and the corresponding free energy is computed via the Ohta-Kawasaki free energy functional.

The improved string method consists of a two-steps iterative process: first the energy of the configurations is minimized to compute the evolution of the string, i.e, solving $\partial m(x, s) / \partial t$, afterwards, the configurations along the string are redistributed such that the chosen parametrization is fulfilled again. In this case, we use the equal arc-length parametrization described by Weinan et al. [112].

To start the iteration procedure, an initial guess for the string must be defined. We construct this initial guess using linear interpolation between the two end-states to obtain the values of $m(x)$ for the configurations along the string at equally spaced distances, $s_{i}=i . \Delta \hat{S}$, as

$$
\mathfrak{m}\left(\mathbf{x}, s_{\mathfrak{i}}\right)=\left(1-s_{\mathfrak{i}}\right) \cdot \mathfrak{m}(\mathbf{x}, s=0)+s_{i} \cdot \mathfrak{m}(\mathbf{x}, s=1) \quad 1<\mathfrak{i}<M
$$

where $\Delta \hat{S}=1 / M$ and $M$ is the number of configurations in the string (including the end states).

Besides the linear interpolation, a different starting set of configurations can be defined if additional information about the transition pathway is available and can be used to set the initial guess closer to the MFEP, reducing the number of iterations. However, the string method is a local optimization procedure and the choice of a particular initial string biases the calculation towards one of the possible pathways [114].

Once an initial path is set, we proceed with the minimization of the free energy of the string (first step in the iteration process, also referred to as the string update) where we deploy the simple forward Euler scheme to solve the 
partial differential equation defined by the Cahn-Hilliard dynamics

$$
\frac{\partial m(\mathbf{x}, s)}{\partial t}=\triangle \frac{\delta F}{\delta m(\mathbf{x}, s)} \equiv \mu_{e f f}(\mathbf{x}, s)
$$

The choice of the Cahn-Hilliard dynamics is based on the interest of investigating paths that correspond to possible physical time-evolutions of the DSA process. Obviously, the diffusive dynamics of the polymers locally conserves the order parameter. Therefore, the driving force for the time-evolution of every configuration is the effective chemical potential, $\mu_{\text {eff }}$.

After the update, the distance between neighboring configurations along the string $\Delta \tilde{s}_{i}$ is computed as

$$
\begin{aligned}
\Delta \tilde{s}_{i} & =\left|m\left(\mathbf{x}, s_{i}\right)-m\left(\mathbf{x}, s_{i-1}\right)\right| \quad \text { for } \quad 1<i \leqslant M \\
& \simeq \sqrt{\sum_{j=0}^{N}\left(m\left(j, s_{i}\right)-m\left(j, s_{i-1}\right)\right)^{2}}
\end{aligned}
$$

and the total arc-length of the string, $\mathbf{S}=\sum_{i} \Delta \tilde{s}_{i}$, is used to normalize those distances as

$$
s_{i}=\frac{1}{S} \sum_{k=2}^{i} \Delta \tilde{s}_{k} \quad \text { for } \quad 1<i \leqslant M \quad \text { thus } \quad 0<s_{i} \leqslant 1
$$

For the study of transition paths where one of the end states is not metastable, the corresponding unstable end point of the string should not be included in the update, thus its free energy remains unchanged and the pathway away from that unstable state can be investigated via this method. Note that the path from an unstable state to the nearest metastable state can simply be obtained by the Cahn-Hilliard dynamics starting from the unstable state.

The updated string with the corresponding distances $s_{i}$ is the input for the third-order spline interpolation deployed to reestablish the equal arc-length parametrization, i.e., $\left|\mathrm{m}\left(\mathbf{x}, s_{\mathfrak{i}}\right)-\mathrm{m}\left(\mathbf{x}, s_{i-1}\right)\right|=\Delta \hat{S}=$ const. This reparametrization of the configurations along the string is the second step in the iteration process.

Inaccuracies due to the interpolation of the order parameter can cause deviations from the mass conservation constraint imposed by the conserved dynamics, i.e., $\int \mathrm{d} \mathbf{x} \delta \mathrm{m}(\mathbf{x})=\int \mathrm{d} \mathbf{x m}\left(\mathbf{x}, s_{i}\right)-\int \mathrm{d} \mathbf{x m}\left(\mathbf{x}, s_{j}\right)=0$. In order to minimize these deviations due to interpolation, the string is discretized using a high number of configurations, i.e., $\mathbf{N}_{\mathrm{s}}=118$ and we use again the linear operator $\mathbf{P}$ described in section 4.1.1 (cf. Eqn 4.12), $\mathbf{P}=(\mathbf{I}-\mathbf{1} / \mathrm{N})$ where $\mathrm{N}$ is the dimension of $\mathrm{m}(\mathbf{x}, \mathrm{s})$. 
Thus, after the redistribution every configuration is projected using the aforementioned operator $\hat{m}_{s}(\mathbf{x})=\mathrm{m}(\mathbf{x}) \mathbf{P}$ and the iteration process is performed until the condition that defines the MFEP is fulfilled.

$$
\left(\nabla\left[\mathfrak{m}(\mathbf{x}, \mathrm{s}) \nabla \frac{\delta \mathcal{F}}{\delta \mathfrak{m}(\mathbf{x}, \mathbf{s})}\right]\right)^{\perp}=0 \text { for all points, } \mathbf{x}
$$

That condition is equivalent to requiring that the perpendicular component of the aforementioned effective chemical potential vanishes [113]

$$
\left(\mu_{\mathrm{eff}}(\mathbf{x}, \mathrm{s})\right)^{\perp}=0 \text { for all points, } \mathbf{x}
$$

If the procedure converges, i.e., $\mathfrak{m}(x, s)$ does no longer change with iterations, the converged string fulfills the previous conditions, Eqs. 4.17 and 4.18. Therefore, instead of monitoring Eq. 4.17 or the convergence of $m(x, s)$ we employ the simpler criterion that the free-energy along the path no longer changes.

Once the MFEP is obtained, we can compute the free energy barriers and access the mechanism of the transition between the states at the end points of the string. The computation of the free energy and the chemical potential using the Ohta-Kawasaki model is not required in any of the two steps of the iteration process, therefore its calculation can be performed at the end of the procedure or for a particular string of interest, which reduces the computational cost of the string method using the continuum model. As a consequence, using $\mu_{\text {eff }}$ instead of $\mu$ as the driving force during the update of the string is not only computationally simpler but also is more physically because the path follows a realistic dynamics.

\subsubsection{Numerical computation of MFEP}

We investigate the annihilation pathway of a dislocation dipole towards a defectfree lamella via the string method using the Ohta-Kawasaki model to describe the block copolymer system and model-B dynamics to update each one of the configurations along the path towards the minimum free-energy path (MFEP). For the latter investigation, we select values of the model parameter that correspond to the intermediate and weak segregation regimes. Previous data is available for MFEP of this very same defect at such values that enable us to compare our findings $[57,74,116]$. Here, we consider a 2D system with periodic boundary conditions of dimensions $\mathrm{L}_{X} / \mathrm{L}_{\mathrm{O}}=7$ and $\mathrm{L}_{Y} / \mathrm{L}_{\mathrm{O}}=5.3$ where the equilibrium lamellar spacing, $\mathrm{L}_{\mathrm{O}}$, corresponds to 36 grid cells of a collocation lattice with $\mathrm{N}=48.384$ cells. 
Figure 4.13 presents the converged path for different values of $\chi N$ and the corresponding values of the model parameter $\tilde{\alpha}$. The path shows the freeenergy difference of the configurations along the path with respect to the initial configuration of the string, i.e., $\mathcal{F}[\mathrm{m}(\mathrm{s}=0)]=-1081.45$ for the case of $\chi N=28.0$. Given this choice of the reference state for the free-energy differences, the highest free energy barrier is simply $\Delta \mathrm{F}$ at the corresponding maximum of the path, i.e., $\Delta \mathrm{F}_{\mathrm{b}}=0.9075$ at $\mathrm{s}=0.179$ for $\chi \mathrm{N}=28$. For the excess free-energy of the defect, $\Delta \mathrm{F}_{\mathrm{d}}=\mathcal{F}[\mathrm{m}(\mathrm{s}=0)]-\mathcal{F}[\mathrm{m}(\mathrm{s}=1)]$, the path shows the corresponding negative values that nevertheless allow a straightforward comparison as the segregation degree changes, i.e., $\mathcal{F}[\mathrm{m}(\mathrm{s}=1)]=-1088.16$ and thus $\Delta \mathrm{F}_{\mathrm{b}}=6.708$ for $\mathrm{XN}=28$.

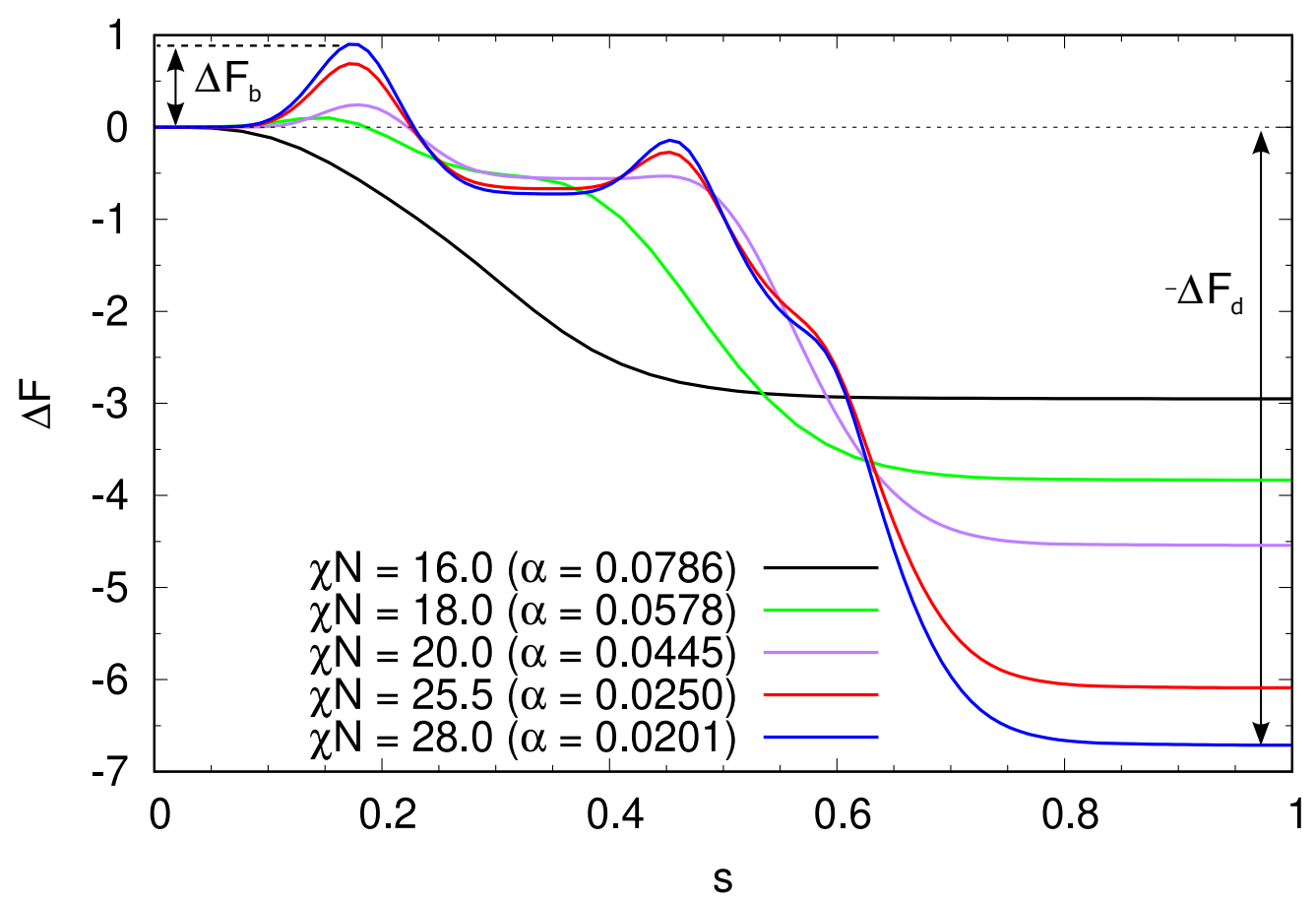

Figure 4.13: Minimum free-energy path (MFEP) between defective structure, $s=0$, and lamellar phase, $s=1$ obtained via the Ohta-Kawasaki model. As the segregation decreases, the MFEP changes from a two-barrier pathway as for $\chi N=28$ towards a one-barrier path as for $\chi N=16$. Defect excess free energy $\Delta \mathrm{F}_{\mathrm{d}}=\mathcal{F}[\mathrm{m}(\mathrm{s}=0)]-\mathcal{F}[\mathrm{m}(\mathrm{s}=1)]$ and the highest free-energy barrier $\Delta \mathrm{F}_{\mathrm{b}}$, both in energy units of the continuum model, are indicated for $\chi \mathrm{N}=28$.

Different barriers along the pathway are observed as $\chi N$ decreases and the free energy landscape becomes more smooth. Two barriers characterize the MFEP at $\chi N=28$ and 25.5, whereas for $\chi N=18$ and 16 only one barrier of 
smaller free energy hinders the transition of the defect towards the lamellar phase.

We will analyze these results as follows: first, we exploit the available results to compare the annihilation pathway at $\chi \mathrm{N}=28$ as well as the decay of the free-energy barrier when $\chi \mathrm{N}$ approaches the limit of defect meta-stability, i.e. $\chi \mathrm{N}^{*}$ or the corresponding $\tilde{\alpha}^{*}$. Afterwards, we study the changes that the defect configuration undergoes in the vicinity of the meta-stability limit, $\chi \mathrm{N}^{*}$ and compare how the annihilation mechanism changes and discuss to what extend the results can be related to the findings of the fluctuation mode analysis.

The MFEP for this transition has been previously described as a path with two barriers and one shoulder in 3D calculations at $\chi \mathrm{N}=28$ and 30 using SelfConsistent Field Theory (SCFT) and at $\chi \mathrm{N}=25$ using Monte Carlo simulations and a soft, coarse-grained model [116] $]^{\mathrm{ii}}$. Our findings of Figure 4.13 correspond to a pathway with the same features and the intermediate configurations depict the same changes in the morphologies along the path.

In addition, Takahashi et al. [74] have obtained the transition pathway between a dislocation dipole with defect cores of opposed A-B domains and a lamellar phase, in 2D calculations via SCFT at $\chi N=25$ describing a path with one barrier and one shoulder. Such dislocation dipole corresponds to the intermediate meta-stable state between the two barriers of our calculations, i.e., the configuration at $s \simeq 0.35$ in Fig. 4.13, therefore the MFEP obtained with the Ohta-Kawasaki model is in qualitative agreement with previous 2D SCFT calculations as well.

For a more detailed comparison of our findings with the results from SCFT calculations of $\mathrm{Li}$ et al. [57], we consider a film of thickness $\mathrm{D}=0.8 \mathrm{~L}_{\mathrm{O}}$ and we extend our 2D calculations to compute the dimensionless free-energy difference along the pathway per film thickness, i.e., $\Delta f=R_{e O} \Delta F / D k_{B} T \sqrt{\bar{N}}$. In this extrapolation of our purely 2D calculations to a thin film, we assume top and bottom surfaces of the thin film are identical and all the configurations are transitional invariant in the $z$ direction, as considered by Li et al. However, in a 3D thin film these assumptions may not always hold, particularly if the substrate has any preference towards the A- or B-domains of the block copolymer.

The energy and length scales of the continuum model are used for consistency of units, $\eta_{0}$ and $\xi_{0}$ respectively

\footnotetext{
ii This model, known by the acronym TICG, relies on a particle based description of the polymer chains but adopts the Hamiltonian used in Self-Consistent Field Theory
} 


$$
\begin{aligned}
\frac{\eta_{0}}{\sqrt{\overline{\mathcal{N}} k_{\mathrm{B}} T}}=\frac{\sqrt{2 \chi \mathrm{N}-7.1}}{135.58}=\frac{1}{135.58}\left(\frac{48}{\tilde{\alpha}}\right)^{\frac{1}{4}} \\
\frac{\xi_{\mathrm{o}}}{\mathrm{R}_{e \mathrm{O}}}=\frac{1}{\sqrt{3(2 \chi \mathrm{N}-7.1)}}=\frac{1}{\sqrt{3}}\left(\frac{\tilde{\alpha}}{48}\right)^{\frac{1}{4}}
\end{aligned}
$$

For $\chi N=28$, i.e., $\tilde{\alpha}=0.0201$, the equilibrium spacing is $\mathrm{L}_{\mathrm{O}}=17.45 \xi_{\mathrm{o}}$ and the energy and length scales are $\eta_{0}=0.0516 \sqrt{\overline{\mathcal{N}}} k_{\mathrm{B}} \mathrm{T}$ and $\xi_{\mathrm{o}}=0.08256 \mathrm{R}_{\mathrm{eO}}$, therefore the film thickness $\mathrm{D}=0.8 \mathrm{~L}_{\mathrm{O}}=1.15256 \mathrm{R}_{e \mathrm{O}}$. Hence, considering the aforementioned values of $\Delta \mathrm{F}_{\mathrm{b}}=0.9075$ and $\Delta \mathrm{F}_{\mathrm{b}}=6.708$, we obtain the dimensionless free-energy differences per film thickness

$$
\Delta f_{b}=\Delta F_{b} \frac{\eta_{0} / \sqrt{\mathcal{N}} k_{B} T}{D / R_{e O}}=0.04063 \text { and } \Delta f_{d}=\Delta F_{d} \frac{\eta_{0} / \sqrt{\mathcal{N}} k_{B} T}{D / R_{e O}}=0.3003
$$

Considering the latter mapping between the units of the Ohta-Kawasaki model and the coarse-grained invariants, the MFEPs obtained can be re-expressed. It is important to mention the aforementioned mapping requires further modifications for more accurate results at the values of the segregation degree investigated herein.

Figure 4.14 presents the pathways obtained via Ohta-Kawasaki in dimensionless free-energy units. Data for the pathway at $\chi N=28$ obtained from the continuum model as well as from SCFT calculations is available and serves as a reference for comparison. To highlight the merging of domains as the morphology changes along the path, only B domains in the configuration (blue) are depicted in Figure 4.14a. A two-barriers pathway is described in both cases where four configurations characterize the annihilation mechanism: the highest freeenergy barrier, see Fig. $4.14 \mathrm{~b}$ (a) $\mathrm{s} \simeq 0.178$, corresponds to the removal of one defect core via the connection of the corresponding A domains. A metastable configuration between the two barriers, $s \simeq 0.339$ in Fig. $4.14 \mathrm{~b}(\mathrm{~b})$, corresponds to dislocation of an opposing A-B defect cores where the remaining A defect core (white) vanishes at the next saddle point at, $s \simeq 0.466$ in Fig. $4.14 b$ (c). After that last saddle point, a configuration with two B defect cores remains and is presented in Fig. 4.14b (d) which corresponds to the shoulder in the pathway at $s \simeq 0.585$. The latter four configurations describing the annihilation mechanism, are in qualitative agreement with the prediction of SCFT calculations.

On the other hand, in Figure 4.15 we present the change of the highest freeenergy barrier $\Delta f_{b}$ for different values of $\chi N$ as well as of the defect excess free 
(a) Dislocation dipole defect investigated via MFEP using Ohta-Kawasaki, $s=0$

(b) MFEP via Ohta-Kawasaki
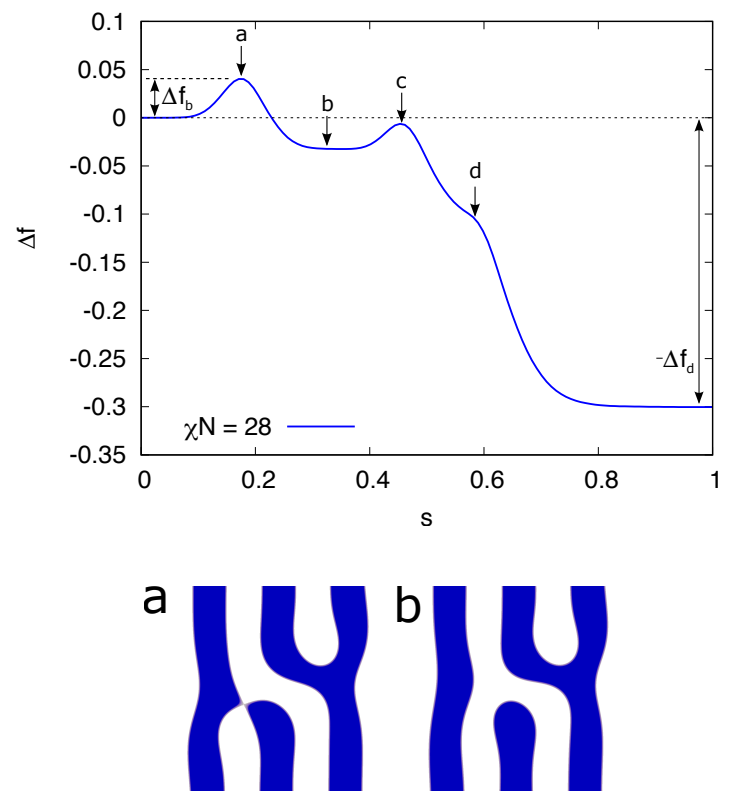

C

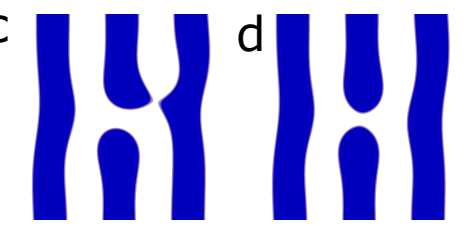

(c) MFEP via SCFT
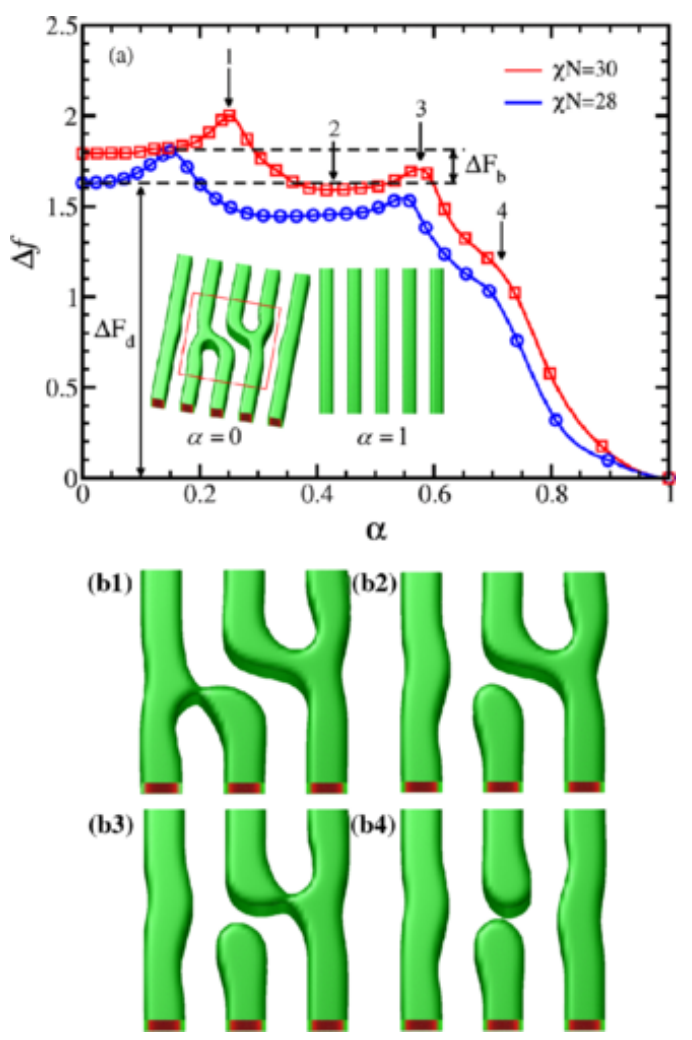

Figure 4.14: Minimum free-energy path (MFEP) between defective structure, $\mathrm{s}=0$, and lamellar phase, $\mathrm{s}=1$. (a)A central portion indicated by the dashed box is used to depict changes of the defect along the path. (b) Characteristic configurations along the path are indicated by numbered arrows and the corresponding snapshots are shown below. Defect excess free energy $\Delta f_{d}$ and the highest free-energy barrier $\Delta f_{b}$ are indicated for this pathway as well. Dimensionless free-energy differences $\Delta f=R_{e O} \Delta F / D k_{B} T \sqrt{\mathcal{N}}$ are per film thickness $\mathrm{D} / \mathrm{R}_{e \mathrm{O}}$. (c) The annihilation path described by the MFEP via the continuum model is in agreement with 2D-MFEP results from SCFT. Figure reproduced with permission from [57]. 
energy $\Delta f_{d}$. Both free-energy differences decrease as the segregation degree decreases but $\Delta f_{b}$ vanishes at $\chi N^{*}=16$, i.e., the limit of defect meta-stability predicted via the Ohta-Kawasaki model, whereas $\Delta f_{d}$ remains large and finite at that limit. This observation is in agreement with the results obtained via SCFT [57] confirming that defects do not form due to thermal fluctuations. The value of $\chi \mathrm{N}^{*}=16$ predicted via the Ohta-Kawasaki model is very close to the value of $\chi \mathrm{N}^{*} \simeq 18$ predicted via SCFT, given the differences in the models and deviations due to the extrapolaton used in SCFT with values far from $\chi \mathrm{N}^{*}$ for that prediction.

Besides the qualitative agreement between the two pathways, an important difference should be highlighted regarding the model used for the kinetics of the configurations along the path. Although SCFT provides a more accurate prediction of the free energy, the minimum free energy pathway presented in Figure 4.14c has been computed using Allen-Cahn dynamics (model A) on the chemical potential fields along the string and thus, the corresponding configurations do not satisfy the mass conservation constraint. On the contrary, the pathways presented herein are obtained via Cahn-Hilliard dynamics (model B) deployed to minimize the Ohta-Kawasaki free-energy functional for every configuration along the string, hence, all the configurations satisfy the mass conservation constraint providing a more precise description of the changes of the morphology along the pathway and not only at local extrema and saddle points of the string where the same configuration is obtained via both models, i.e., Allen-Cahn and Cahn-Hilliard.

The agreement between the MFEP and the configurations describing the annihilation mechanism obtained via the continuum model, SCFT and particlebased simulations, serves to conclude that single-chain dynamics do not give rise to additional bottlenecks in the transition pathway and therefore the choice of a collective variable to describe the transition states, i.e, the order parameter, is a valid choice in combination with the Ohta-Kawasaki free-energy functional to investigate this transition.

\subsubsection{Results and comparison}

So far we have shown the Ohta-Kawasaki model can be used together with the string method to describe the defect-removal pathway, in agreement with a more accurate free-energy functional for the block copolymer system. Now, we use the string method and the continuum model to describe the changes in the local composition of the defective morphology at the vicinity of defect meta-stability, i.e., for $\chi N \simeq \chi N^{*}$.

The MFEP for values of $\chi \mathrm{N}$ around that spinodal limit, are presented in Fig- 
(a) Ohta-Kawasaki

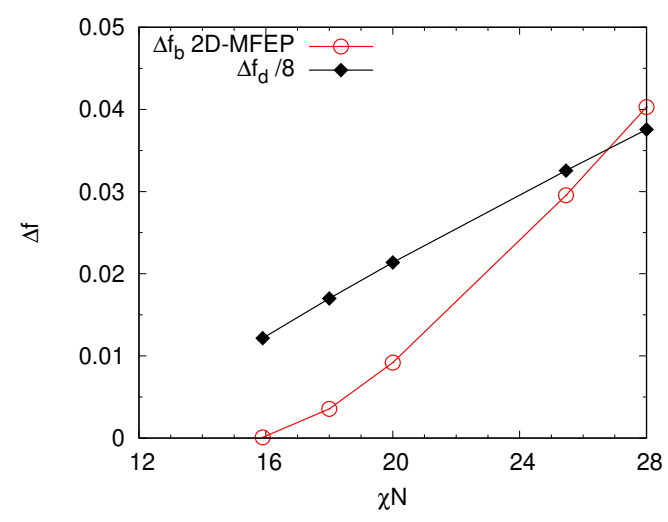

(b) SCFT

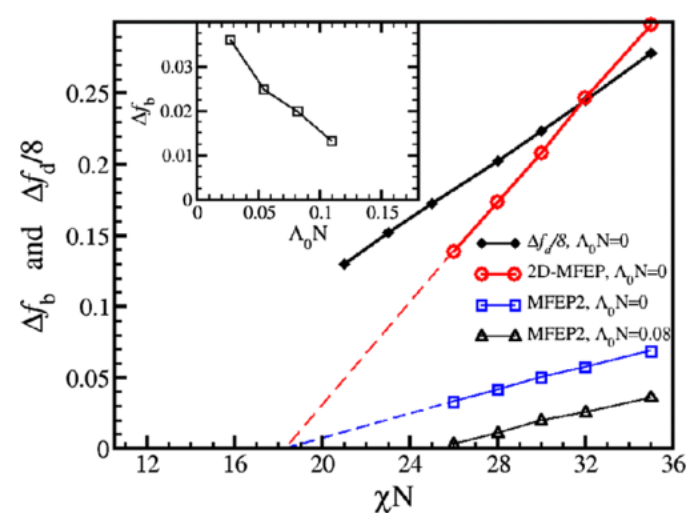

(c) Quantitative comparison

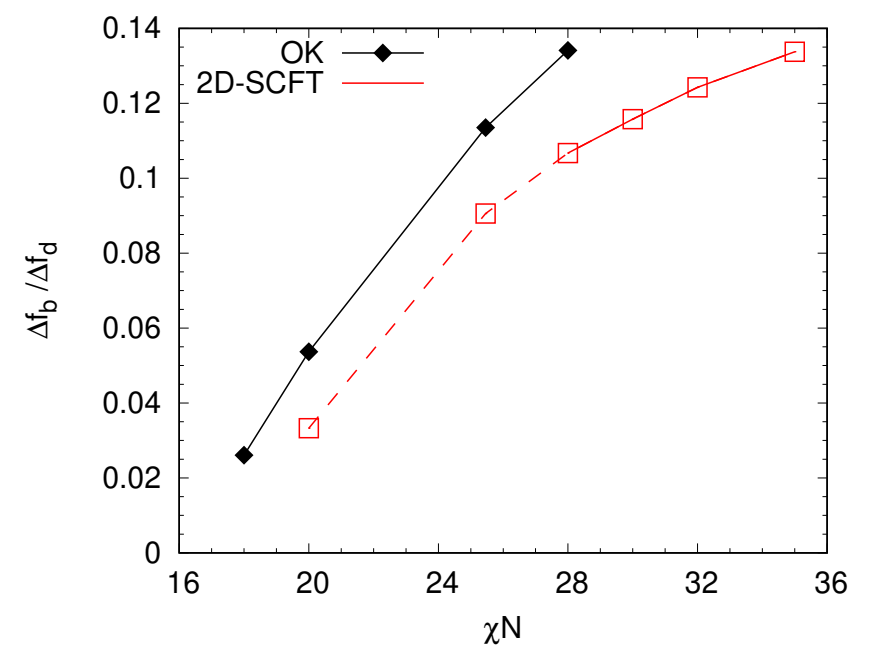

Figure 4.15: Defect excess free energy $\Delta f_{d}$ and highest free-energy barrier $\Delta f_{b}$. (a) As the segregation decreases, the free-energy barrier decreases and vanishes at the limit of defect meta stability predicted via the Ohta-Kawasaki model: $\chi \mathrm{N}^{*}=16$. Defect excess free energy $\Delta \mathrm{f}_{\mathrm{d}}$ decreases as well but remains large and finite at that limit. (b) This results are in qualitative agreement with SCFT calculations for the same defect topology at $\Lambda \mathrm{N}=0$ representing a neutral surface preference for A-B domains where the predicted value of $\chi \mathrm{N}^{*} \simeq 18$ is close to the prediction of the continuum model. Figure reproduced with permission from [57]. (c) The ratio between the defect free energy and the free-energy barrier provides a quantitative comparison of the prediction of both models. 
ure 4.16 where the model parameter $\tilde{\alpha}$ is used instead of $\chi N$ to identify each string. For this calculations, the configurations at the extremes of the string, i.e., $s=0$ and $s=1$, are not updated via the Cahn-Hilliard model as the MFEP is computed. The free-energy change at the start of the string, i.e., $\Delta f / \Delta s$ at $s=0$ is positive for $\tilde{\alpha}=0.0786$ indicating the starting configuration is metastable at this value of $\tilde{\alpha}$. On the contrary, the negative slope for $\tilde{\alpha}=0.0788$ at $s=0$ implies the configuration is no longer stable and would spontaneously evolve to the defect-free lamella.

The change in local composition, $\left.\delta \mathrm{m}(\mathbf{x})\right|_{s=0}=\partial \mathrm{m}(\mathbf{x}) /\left.\partial s\right|_{s=0} \Delta s$, is computed using the first derivative from the third-order spline interpolation, i.e., $\partial \mathrm{m}(\mathbf{x}) / \partial s$, used during the string calculations. In Figure 4 .16a we present $\left.\delta \mathrm{m}(\mathbf{x})\right|_{s=0}$ for the three values of $\tilde{\alpha}$ around the spinodal limit and how this local changes affect the defect configuration $s=0$. The factor $\Delta s$ has been arbitrarily chosen as $\Delta s \simeq 0.43=50 \mathrm{ds}$, for a better graphical representation of the changes in the defect, where ds is given by the discretization of the string $N_{s}=118$ as ds $=1 /\left(\mathrm{N}_{\mathrm{s}}-1\right)$.

In the case of the unstable defect $\tilde{\alpha}=0.0788$, the major changes in the local composition occur between the defect cores and the intermediate defective lamella. As a result, interfaces break and merge resulting in defect annihilation. In contrast, local composition changes in the meta-stable configuration $\tilde{\alpha}=0.0786$, are not localized around the AB interfaces of the structure at $s=0$ (depicted by the black contour lines) and do not promote significant changes of the defect cores but an undulation of all the structure.

This local changes of the defective configuration at the onset of the annihilation pathway, can be also predicted via the aforementioned fluctuation mode analysis. At the limit of the metastability, the local composition changes that lead to defect annihilation, i.e., $\left.\delta m(\mathbf{x})\right|_{s=0}$ for $\alpha>\alpha^{*}$ in Fig. 4.16a, should be also described by the low-lying fluctuations (or soft modes) of the corresponding meta-stable configuration. To prove this, we compute the fluctuation modes for the defect configuration at the last value where the defect is stable $\alpha \simeq \alpha^{*}=0.0787$ and study how those fluctuations change the AB interfaces of the defect. Three soft modes promote defect removal and Figure 4.17 shows these annihilation modes. Among them, the one associated with the lowest free-energy change, i.e., with the smaller value of $\lambda^{k}$ thus $k=2$, corresponds to a soft mode of the lamellar structure. This can be confirmed by a comparison of the fluctuating $\mathrm{AB}$ interfaces due to the mode $k=2$ in Figure 4.17 (red contour lines) with respect to the interface fluctuations in the lamellar modes (see mode $k=2$ in Figs. 4.9 and 4.8). Considering mode $k=2$ is a fluctuation characteristic of the defect-free structure, we discard this mode for our comparison and focus on the remaining two modes. 


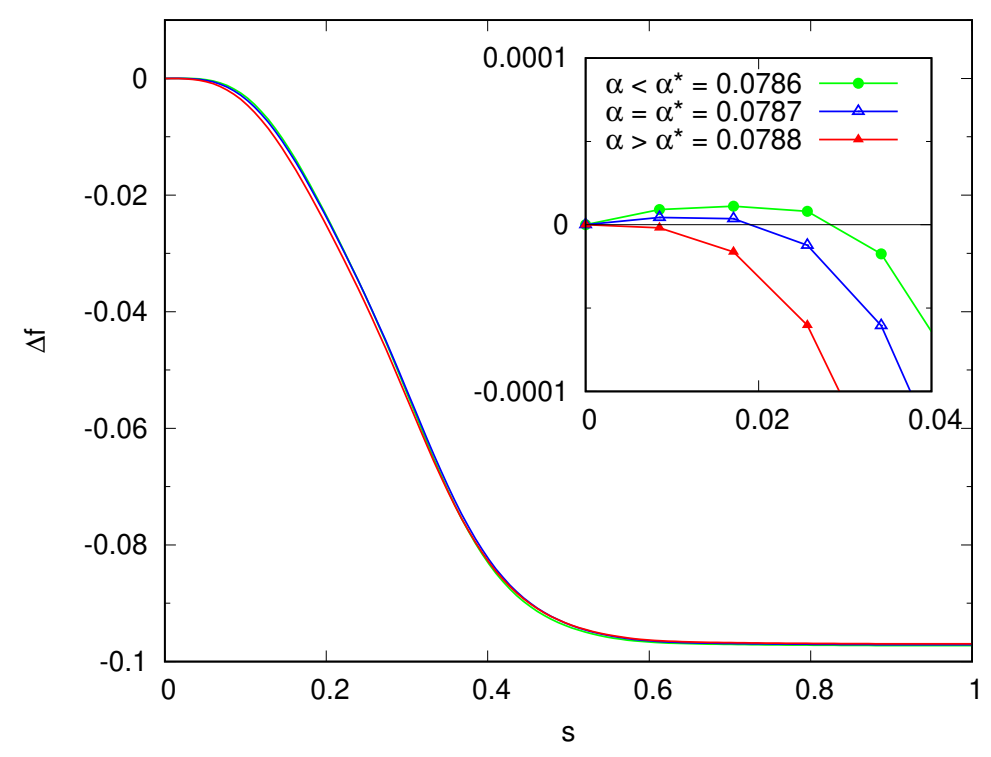

(a) Top row: $\delta \mathfrak{m}(\mathbf{x})=\partial \mathfrak{m}(\mathbf{x}) /\left.\partial s\right|_{s=0} \Delta s \quad$ Bottom row: $\mathfrak{m}(\mathbf{x})=\left.\mathfrak{m}\right|_{s=0}+\delta \mathfrak{m}(\mathbf{x})$
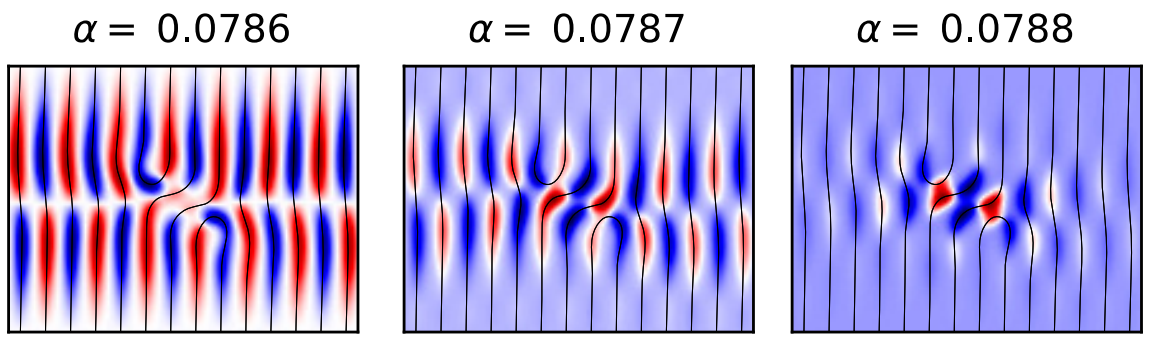

$\delta m(x)$
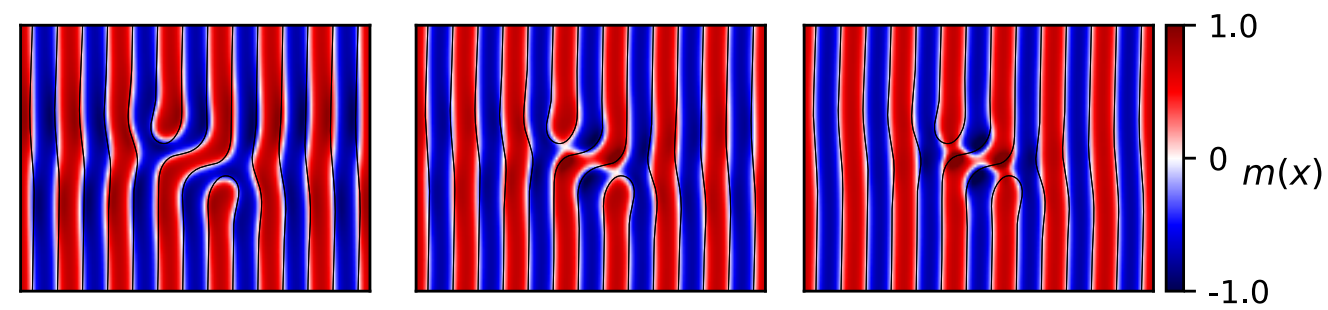

Figure 4.16: Minimum free-energy pathway in the limit of defect meta stability. Pathways in the vicinity of that limit and beyond, i.e., $\alpha<\alpha^{*}$ and $\alpha>\alpha^{*}$ respectively, are computed to obtain the local composition changes that the defect configuration, i.e., $s=0$ undergoes at the onset of the annihilation pathway. Color maps (top row) depict those local changes, i.e., $\delta \mathrm{m}(\mathbf{x})=\partial \mathrm{m}(\mathbf{x}) /\left.\partial s\right|_{s=0} \Delta s$, for the limiting values of $\alpha$ investigated. Major changes are located near the $A B$ interfaces of the defect configuration (black contour lines). With the resulting configuration due to those local changes, i.e., $m(\mathbf{x})=\left.m\right|_{s=0}+\delta m(x)$, (bottom row) changes in the topology of the defect can observed. 

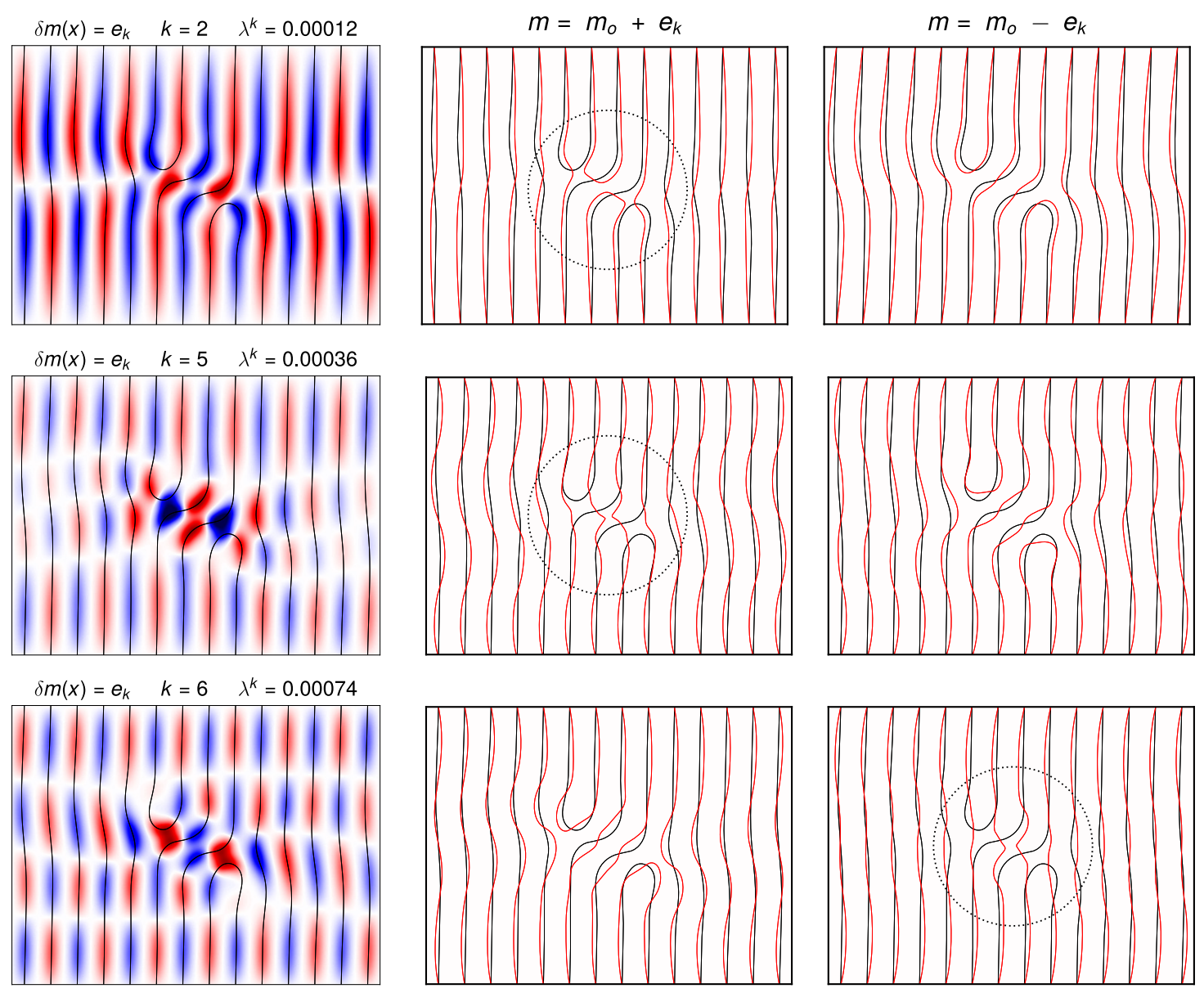

Figure 4.17: Annihilation modes describing local composition changes $\delta \mathrm{m}(\mathbf{x})$ obtained via the fluctuation mode analysis. Among the low-line fluctuation modes of the defect at the limit of meta stability, i.e., $\alpha=0.0787$, the modes $k=2,5,6$ lead to defect annihilation. Changes in the AB interfaces due to fluctuations (red contour lines) show how defect cores merge and connect promoting the formation of lamellar phase, as indicated by the black dotted circles. Same sign in the local composition changes at the apex of the defect cores (blue for $k=5$ and red for $k=6$ ) is consistent with the A-domains defect cores in the structure. These modes are referred to as symmetric annihilation modes. Black contour lines depict $\mathrm{AB}$ interfaces of the unperturbed defect at $\alpha=0.0787$. 
The more localized annihilation modes, $k=5$ and $k=6$, affect the interfaces of the defect cores in a similar fashion. However, mode 6 cause the undulation of the $\mathrm{AB}$ interfaces of the entire structure with a higher frequency than mode 5 , with the associated higher free-energy cost. Therefore, we compare mode 5 to the local composition changes observed with the string method, i.e., $\left.\delta \mathrm{m}(\mathbf{x})\right|_{s=0}$ for $\alpha=0.0788$ in Fig. 4.16a, and we confirm they provide the same description of the local composition changes around the defect cores that lead to the merging of $\mathrm{AB}$ interfaces and the consequent annihilation of the defect.

Based on the latter findings, we confirm that the fluctuation mode analysis (FMA) can describe the local composition changes that will take place at the onset of defect annihilation at the spinodal value of $\chi \mathrm{N}^{*}$ representing the limit of defect meta-stability, or the corresponding values of the model parameters $\tilde{\alpha}$ in the case of a symmetric block copolymer. The following criteria can be highlighted to identify the fluctuation mode promoting defect annihilation: the mode involves mayor composition changes localized around the interfaces of the defect, the mode does not correspond to the fluctuations of the lamellar structure and the mode is part of the soft modes of the defect, therefore has the lowest possible free energy change among all the possible localized modes in the space of eigenvectors of the Hessian matrix of the defect.

In comparison to the string method, the fluctuation mode analysis is only valid for meta-stable states where the harmonic approximation holds as a valid estimation for the free-energy basin whereas the string method enables us to describe transition pathways between two states regardless of their stability. However, the computational effort required to deploy the fluctuation mode analysis is lower compared to the calculations required for the string method. The later involves solving the partial differential equation for the Cahn-Hilliard dynamic of $\mathrm{N}_{s}$ configurations during the entire iteration process and computing the free energy for the $N_{s}$ configurations for at least one iteration step, among other less expensive calculations. Even when the use of parallel programming can significantly increase the performance and efficiency of those calculations, the implementation of the string method implies more effort. On the contrary, the FMA involves solving the Cahn-Hilliard equation for one configuration, the calculation of the Hessian matrix (which is of the same complexity as the calculation of the free energy) and perform the matrix decomposition to obtain the corresponding eigenvalues and eigenvectors. A variety of optimized linear algebra libraries are available for this latter task, making the FMA more readily available.

On the other hand, we have only identified the fluctuation mode that can be used to predict how the changes in the local composition of the meta-stable defect will be at the onset of the defect annihilation close to the spinodal tran- 
sition, i.e., for $\tilde{\alpha} \simeq \tilde{\alpha}^{*}$. The question remains if we can use the same approach to identify that fluctuation mode in defective configurations far from the limit of metastability, which is a common case for experimental conditions in the directed self-assemble of block copolymers. We address that open question in the next section.

\subsection{Dominant modes in defect annihilation}

We have identified several annihilation modes at the limit of defect meta-stability, $\chi N^{*}$. The agreement between the local composition changes predicted via the minimum free-energy pathway (MFEP) and one of those annihilation modes enables us to label which mode describes the changes that the configuration undergoes at the onset of the defect annihilation process at that value of $\chi \mathrm{N}^{*}$. This mode is referred to as the dominant annihilation mode.

In this section we address the question of how those identified modes evolve as the segregation degree of the block-copolymer approaches the region of metastability of the defect, i.e. as $\chi N>\chi N^{*}$. The evolution of the eigenvalue of the most unstable mode has been previously used to predict the spinodal limit of the ordered phases of diblock copolymer in the bulk [117-120], exploiting the symmetries of those phases and performing an analysis of the eigenmodes and eigenvalues in reciprocal space. The same approach could be used to predict the limit of the defect meta-stability via the decay of the eigenvalue associated to the dominant annihilation mode. Therefore, the goal of our numerical calculations is to identify the region of $\chi \mathrm{N}$ where the eigenvalue of the dominant annihilation mode follows a linear behavior and how the eigenvalue of the other annihilation modes evolve as $\chi \mathrm{N}$ changes.

In this regard, we consider a prototypical dislocation defect of opposed AB domain defect cores in a lamellar phase to study the modes of a defective configuration. This configuration is shown in figure 4.18. The fluctuation mode analysis is deployed for this defect at the corresponding value of $\chi \mathrm{N}^{*}=19.61$ and in figure 4.19 we present the three annihilation modes identified.

Among these modes, $k=1$ corresponds to composition changes that are the most localized at the apex of the defect cores and to the lowest free-energy cost due to the lowest value of $\lambda_{k}$. Following the comparison in the previous section, this composition changes are in agreement with the changes of the configuration along the MFEP at $\chi \mathrm{N}^{*}$, from which we confirm mode $k=1$ is the dominant annihilation mode.

To study the evolution of the annihilation modes, including $k=1$, we identify the eigenvectors associated to this modes in the new eigenspace of fluctuations modes that arises as $\chi \mathrm{N}$ changes. If the fluctuation modes are normalized 


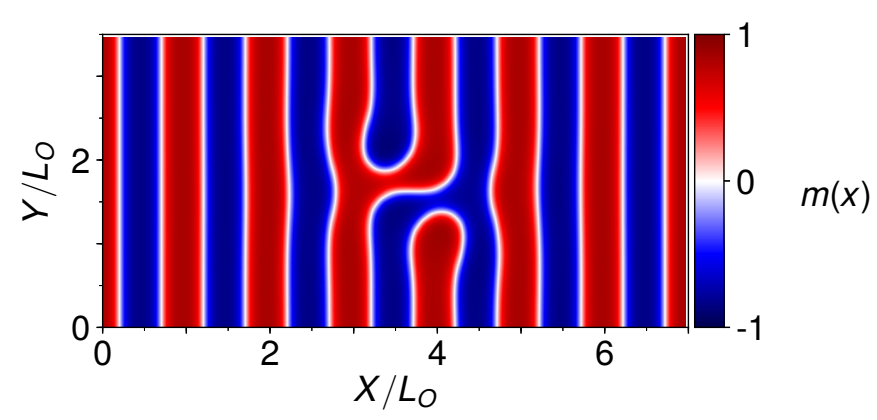

Figure 4.18: Dislocation pair with defect cores of opposed A and B domains of a symmetric block copolymer. The configuration corresponds to the local minimum at $\tilde{\alpha}=0.042$ equivalent to $\chi \mathrm{N}=20.45$. $\mathrm{L}_{\mathrm{O}}=14.15$ is discretized with 12 grid cells in a $2 \mathrm{D}$ lattice of dimensions $\mathrm{L}_{X} / \mathrm{L}_{\mathrm{O}}=7$ and $\mathrm{L}_{Y} / \mathrm{L}_{\mathrm{O}}=3.5$.
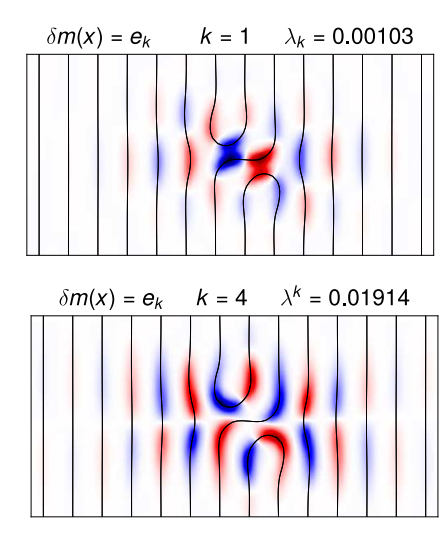

$\delta m(x)=e_{k} \quad k=5 \quad \lambda^{k}=0.02913$

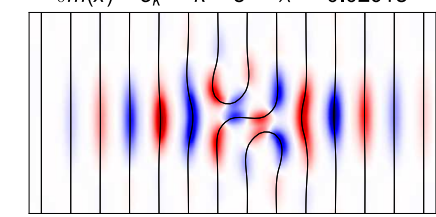

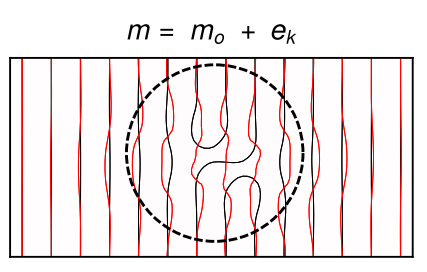
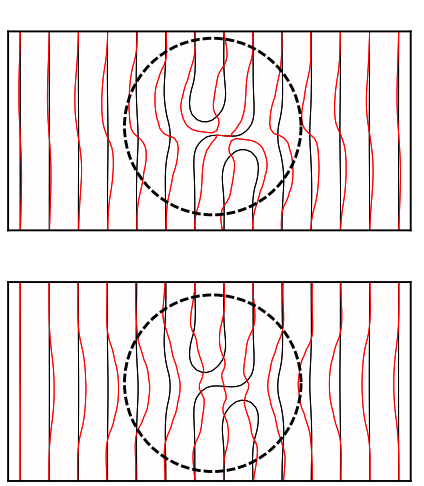
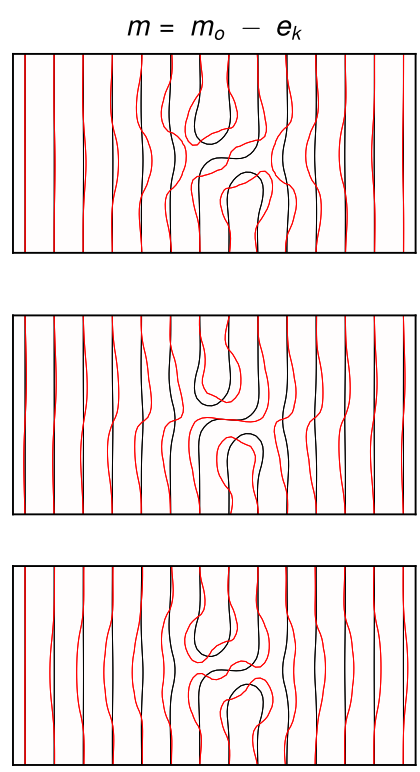

Figure 4.19: Annihilation modes (color map) at the limit of meta-stability of the defect, $\chi \mathrm{N}^{*}=19.612$, equivalent to $\alpha^{*}=0.046512$. Changes in the AB interfaces due to the corresponding fluctuations (red contour lines) show how defect cores merge and connect promoting the formation of the lamellar phase, as indicated by the black dotted circles. Color map depicts every mode $e_{k}=\delta m(\mathbf{x})$ where red and blue correspond to positive and negative values, respectively. Black contour lines depict $\mathrm{AB}$ interfaces of the unperturbed defect.

eigenvectors, the projection of a given mode $e_{k}$ onto another $e_{j}$, i.e., the dot product between them, has a value of one when the two eigenvectors are the same, i.e., $e_{k} \cdot e_{j}=1$ for $e_{k}=e_{j}$ and $\left|e_{k}\right|=\left|e_{j}\right|=1$. 
Therefore, the associated mode at $\chi \mathrm{N}_{i}=\chi \mathrm{N}^{*}+\delta \chi \mathrm{N}$ is identified as the eigenvector in the new space with a projection of one (or the closest to one) and the corresponding change in the eigenvalue of that annihilation mode is obtained. With the same approach, the mode at $\chi \mathrm{N}_{i+1}$ can be identified using the eigenvector at $\chi \mathrm{N}_{i}$ and the eigenvalue of each annihilation mode can be obtained far away from the limit of meta-stability. Figure 4.20 presents the eigenvalue, $\lambda_{k}$, for the three annihilation modes $k=1,4,5$ as a function of $\chi N$.

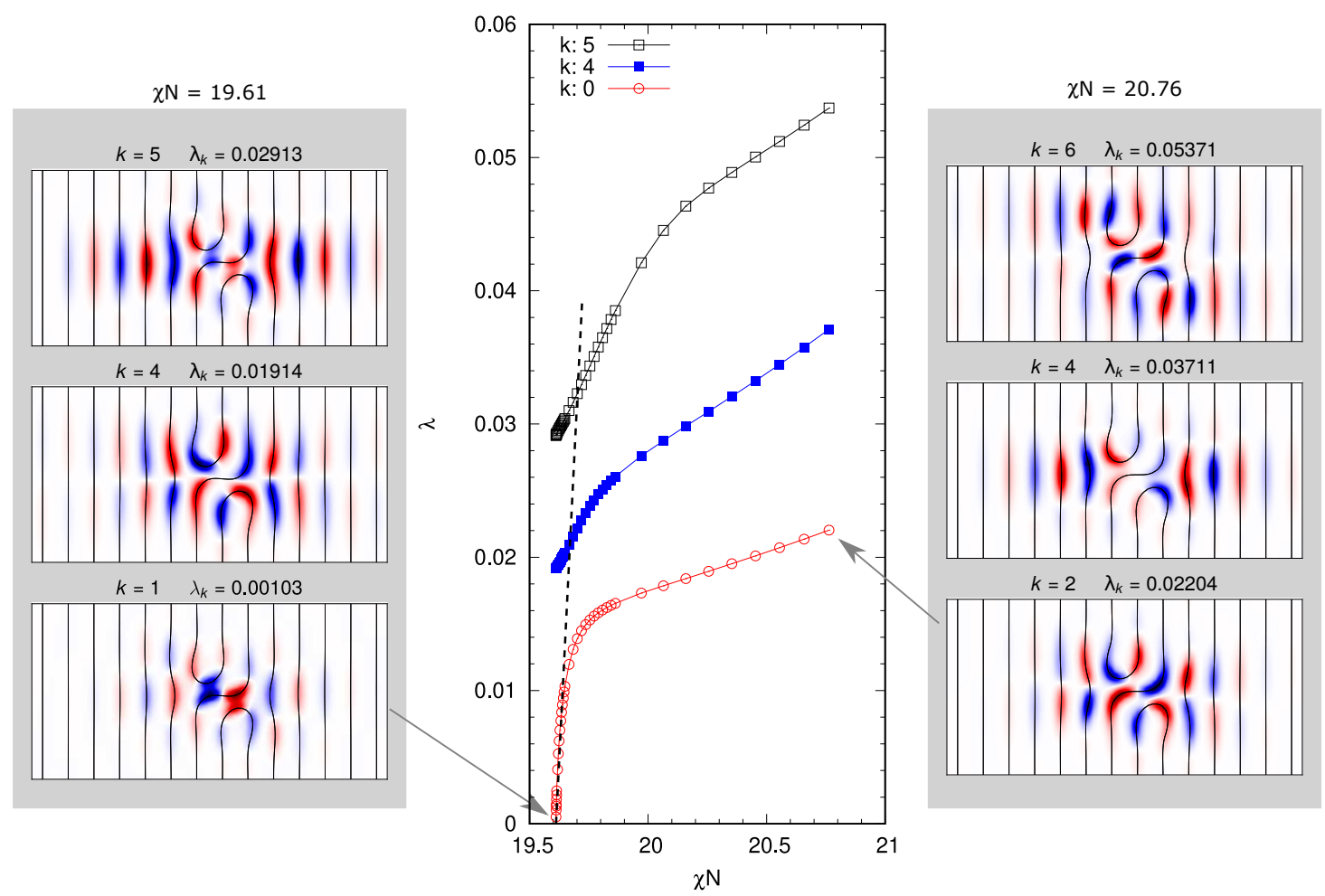

Figure 4.20: Eigenvalue of the annihilation modes as $\chi N$ approaches the defect meta-stability region. Two linear regimes describe the change of those eigenvalues as the free-energy landscape gradually changes: a high-slope regime close to the spinodal limit, $\chi N \rightarrow \mathrm{N}^{*}$, and a low-slope regime in the region of defect meta-stability, $\chi N>\chi N^{*}$. The eigenvalue of the dominant mode, $\lambda_{k=1}$, exhibits the highest slope in the first regime (dashed line) which extends over a very narrow interval of $\chi \mathrm{N}$. k-labels in the plot correspond to the eigenvector index at the spinodal.

The decay of $\lambda$ implies a decrease in the free energy cost of the corresponding composition changes in the meta-stable configuration, $\Delta \mathcal{F}=\mathscr{F}\left[m_{\mathrm{o}} \pm e_{\mathrm{k}}\right]-$ $\mathcal{F}\left[\mathrm{m}_{\mathrm{o}}\right] \sim \lambda_{k}$. Therefore, the fluctuation mode for which the free-energy cost approaches zero faster as $\chi \mathrm{N}$ comes closer to the spinodal limit is the mode 
leading the transition out of the free-energy basin where the configuration lies. This fast decay of $\lambda$ is observed for $k=1$ confirming this is the dominant annihilation mode, which is consistent with the agreement observed with MFEP calculations. Thus, this linear decay of $\lambda_{1}$ (see dashed line in Figure 4.20) can be the one used to predict the value of $\chi \mathrm{N}$ at the spinodal limit.

However, the change of the slope from the fast- to a slow-decay regime of $\lambda_{1}$ occurs very close to the spinodal limit and this high-slope linear regime only extends over a very narrow interval of $\chi N$. The extrapolation using the linear regime in the meta-stable region, i.e., $\chi N \geqslant 20.2$ in Fig. 4.20, does not yield to a valid prediction of $\chi N$ given the significant difference in the slope of the two linear regimes.

As the segregation degree of the block-copolymer approaches the spinodal limit, every eigenvalue decays following two linear regimes: a slow-decay regime in the meta-stable region and a faster-decay regime in the vicinity of the spinodal. The two regimes are observed in every case, despite the slopes of every linear region differ among the three annihilation modes.

The same two-linear regimes and a narrow high-slope linear interval of $\chi N$ are observed for the eigenvalue of annihilation modes of the dislocation dipole studied in the previous section. For that defect, we observed that the decay of the free energy barrier as a function of $\chi \mathrm{N}$ has a different slope close to the spinodal limit compared to that in the meta-stability region (see Figure 4.15a). This change implies different annihilation mechanisms: a simultaneous merging of the two defect cores for $\chi N \rightarrow \chi N^{*}$ and a sequential merging for $\chi N>\chi N^{*}$ (see Figs 4.14 and 4.16). Therefore, considering the rate of change of the highest free-energy barrier with respect to $\chi N$ is not constant, it is reasonable to obtain a limited interval of $\chi N$ for the high-slope linear decay of $\lambda$.

On the other hand, as the segregation increases the annihilation modes become more stiff as sharper interfaces hinder the defect to annihilate and local composition changes promoting interface merging have a higher free-energy cost. In this regard, figure 4.21 shows the local composition changes for the dominant annihilation mode in the two linear regimes. Composition changes highly localized between the apex of the defect cores and the intermediate AB interface are the characteristic feature of the annihilation mode in the high-slope regime (top row, Fig. 4.21). On the contrary, in the low-slope regime (bottom row), the composition changes are located along the AB interfaces instead of at the apex of the defect cores. This localized alternating composition changes along the interfaces correspond to a rotation of the defect pinned to the lamellar structure (see top row, Fig. 4.22).

Figure 4.22 presents the fluctuations of the $\mathrm{AB}$ interfaces due to the eigenvector in the meta-stable region that corresponds to the annihilation modes of 

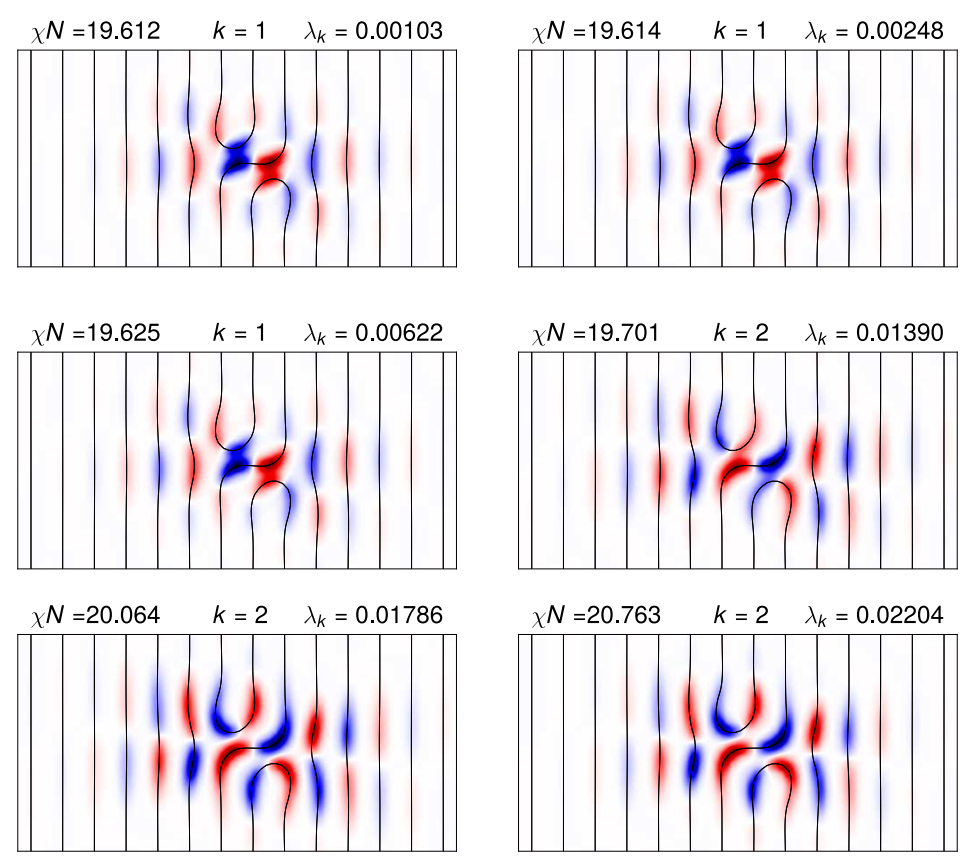

Figure 4.21: Changes in the first annihilation mode as $\chi N$ approaches the defect meta-stability region. Local composition changes are very localized around the apex of the defect cores at $\chi \mathrm{N} \simeq \chi \mathrm{N}^{*}=19.612$, where the different sign in the local composition changes (red/blue) is consistent with the opposed-domains defect cores in the structure. Thus, this is referred to as an anti-symmetric annihilation mode. As $\chi \mathrm{N}$ increases, local composition changes occur along the interfaces of the defect cores and the interface between them.

the defect. We observe that no merging of the interfaces leading to defect annihilation takes place at values of $\chi N>N^{*}$ due to fluctuations associated with the annihilation modes.

An analogy of the two linear regimes for the eigenvalue of the unstable modes to the eigenvalue bands described by Laradji et al. [117-120] and Ranjan et al. [121] can be made, in order to provide a complementary explanation for the change of the slope of the eigenvalue as a function of $\chi N$. The analogy exploits the idea of the mixing of eigenmodes as the slope changes in a similar way as the electronic energy states mix at the band gaps in cristaline solids. The similarity between the annihilation mode $k=4$ at $\chi \mathrm{N}^{*}$ and the mode $\mathrm{k}=2$ at $\chi \mathrm{N}=20.76$ in figure 4.20, suggests that the mix of the first two annihilation modes give rise to the change in the slope observed, and the corresponding changes in the eigenvector presented in figure 4.21. Further analysis and discussions, that fall beyond the scope of the present work, are required to fully exploit this analogy for a complementary understanding of the numerical re- 

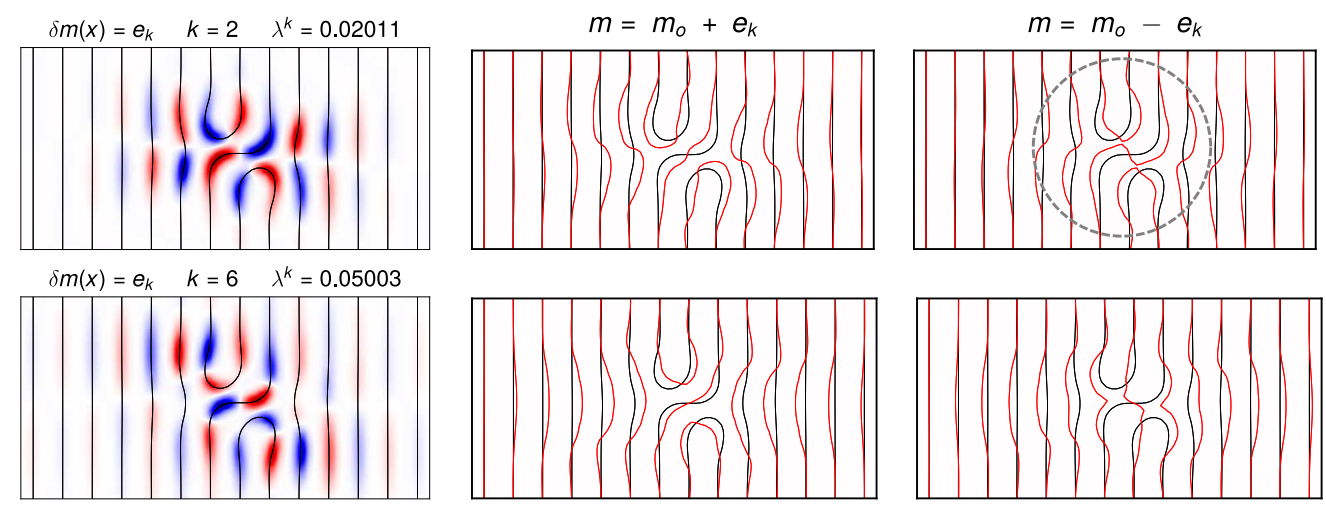

$\delta m(x)=e_{k} \quad k=4 \quad \lambda^{k}=0.03324$
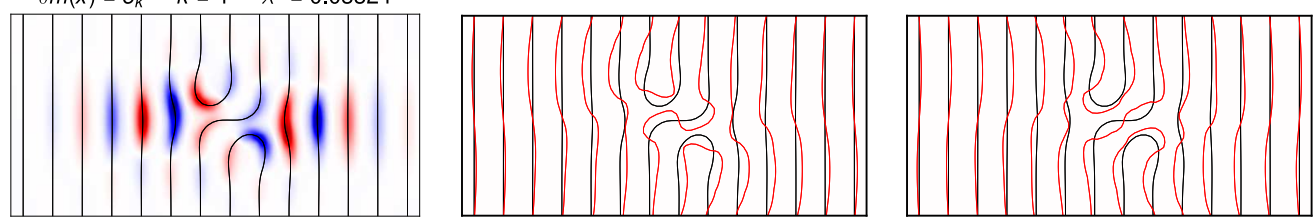

Figure 4.22: The successive projection of the annihilation modes identified at $\chi \mathrm{N}^{*}$ leads to modes $\mathrm{k}=2,4,6$ at $\chi \mathrm{N}=20.76$ far inside the defect meta-stability region. Fluctuations of the $A B$ interfaces due to the those modes (red contour lines) do not promote the formation of a lamellar phase. However, fluctuations of mode $k=2$ deform the $A B$ interfaces bringing the apex of the defect cores close to merging, as indicated by the black dotted circles. Black contour lines depict $A B$ interfaces of the unperturbed defect.

sults.

The results of our numerical study show that although $\lambda$ behaves linearly with respect to $\chi \mathrm{N}$ in the vicinity of the spinodal, it is not feasible to predict the limit of the metastability of the defect based on the behaviour of the corresponding eigenvalue in the metastable region. This is due to the change in the annihilation mechanism as $\chi \mathrm{N}$ and the different slope of the linear behaviour of $\lambda$ in the two regimes observed. Another important aspect to consider in the interpretation of the two linear regimes observed, is the limit of validity of the harmonic approximation to describe the free-energy basin as the free-energy landscape changes. It is expected that for $\chi N>>\mathrm{N}^{*}$ deviations from the harmonic approximation arises and higher order modes in the Taylor expansion of the free energy around the metastable state are required for a more accurate approximation of the free energy changes. 


\subsection{Fluctuation modes in defect metrology in DSA}

Defect detection and characterization are open challenges that must be overcome to meet the requirements of industry and enable directed self-assembly (DSA) of block copolymers as a reliable manufacturing process in the semiconductor industry. Scattering techniques allow the screening of large areas of material, as opposed to Scanning Electron Microscopy (SEM) and Atomic Force Microscopy (AFM), and they provide information about the orientational order and preferred length scales in the material. AFM allows incredible spatial resolution ranging from $1 \mathrm{~nm}[122,123]$ to individual atoms in a single molecule [123]; however, its field of view is usually below $100 \mu \mathrm{m}$. Given this limitation, scanning a wafer for defect identification by using this technique requires several hours considering the typical size of an entire silicon wafer of 450 mm nowadays. SEM has a field of view of $1 \mathrm{~mm}$ maximum [124], thus providing a larger scan area than that of AFM, and a spatial resolution of $1 \mathrm{~nm}$ as of 2017 [122]. In contrast, optical techniques such as light scattering topography enable the rapid inspection of an entire silicon wafer [125] in the order of minutes or even seconds. The maximum theoretical resolution of optical systems is dictated by the diffraction limit given by $\mathrm{d}=0.5 \lambda / \mathrm{NA}$. By considering typical values of the numerical aperture, i.e., $1.0 \leqslant N A \leqslant 1.35$ [126] and the minimum wavelenght of visible light $(380 \mathrm{~nm}$ ), a resolution of $d \simeq 140 \mathrm{~nm}$ can be attained [127]. In practice, however, the resolution achieved ranges from 200 $\mathrm{nm}$ to $500 \mathrm{~nm}$ [126].

The results obtained from scattering experiments are complemented by theoretical predictions of scattering data for improved understanding of the material structure as well as a comprehensive interpretation of the experimental data. In this regard, a theoretical prediction of the scattering pattern of a lamellar structure's thin film of a specific block copolymer has been previously compared to X-ray experimental data [128]. Such a theoretical prediction exemplifies that physics-based models can provide superior analysis of the thermodynamic information behind the complex behaviour of the material under various process conditions.

In this section, we provide theoretical predictions for the structure factor of a defective lamellar structure obtained via the Ohta-Kawasaky model and we compare these predictions with the corresponding defect-free lamella. Subsequently, we study the influence of the fluctuation modes of the defect-free lamella on the structure factor of the mean-field configuration. This information allows us to study the influence of thermal fluctuations on the scattering of the defect and provides insights to develop an alternative procedure for defect detection and characterization from the scattering data of a self-assembled structured obtained via DSA. 
We consider again a dislocation defect with opposed A and B cores as the prototypical metastable defect in our continuum model confined in a $2 \mathrm{D}$ configuration. We study the collective scattering from fluctuations of the composition in this polymer melt, which are fluctuations of the electron density as well. Thus, the scattering intensity I(q) can be conceived as the structure factor of the system $S(q)$ and both terms are equivalent and interchangeable in what follows.

For the computation of $\mathrm{I}(\mathrm{q})$, the order parameter $\mathrm{m}(\mathbf{x})$ is defined in terms of the collective local densities of the block copolymer, and that the amplitude of the scattered wave is proportional to the Fourier transform of those densities. Hence, the complex scattering amplitude $\hat{m}(q)$ and $I(q)$ are computed as

$$
\begin{aligned}
\hat{\mathrm{m}}(\mathbf{q}) & =\mathfrak{F}[\mathrm{m}(\mathbf{x})] \\
\mathrm{I}(\mathrm{q}) & =|\hat{\mathrm{m}}(\mathbf{q})|^{2}
\end{aligned}
$$

The experimentally-observable scattering intensity is composed of the main scattering $I_{m}$, describing the Bragg peaks of the material, and the diffuse scattering $I_{d}$, accounting for thermal fluctuations, i.e., $I(q)=I_{m}(q)+I_{d}(q)$. We consider fluctuations in the continuum model by means of each normal mode $\mathrm{m}(\mathbf{x})=\mathrm{m}_{\mathrm{o}}(\mathbf{x})+\sum_{\mathrm{k}} \varepsilon_{\mathrm{k}} e_{\mathrm{k}}(\mathbf{x})$, and $\mathrm{I}(\mathrm{q})$ corresponds to the average over all fluctuations. In this description, only the values of $\varepsilon_{k}$ fluctuate, and thus, an integral over each mode is deployed to compute that average, thereby yielding expressions for $I_{m}$ and $I_{d}$

$$
\begin{aligned}
& \mathrm{I}(\mathrm{q})=\left\langle|\hat{\mathrm{m}}(\mathbf{q})|^{2}\right\rangle \\
& =\frac{1}{z} \int d \varepsilon_{k}\left(\left|\hat{m}_{o}(q)\right|^{2}+2 \varepsilon_{k}\left|\hat{m}_{o}(q) \bar{m}_{k}(q)\right|+\varepsilon_{k}^{2}\left|\hat{m}_{k}(q)\right|^{2}\right) e^{-\mathcal{F}\left(\varepsilon_{k}\right) / k T} \\
& =\left|\hat{m}_{\mathrm{o}}(\mathbf{q})\right|^{2}+\frac{1}{z} \int\left(2 \varepsilon_{k}\left|\hat{m}_{\mathrm{o}}(\mathbf{q}) \hat{m}_{k}(\mathbf{q})\right|\right)+\frac{1}{z} \int\left(\varepsilon_{k}^{2}\left|\hat{m}_{k}(\mathbf{q})\right|^{2}\right) \\
& =\left|\hat{m}_{\mathrm{o}}(\mathrm{q})\right|^{2}+\sum_{\mathrm{k}}\left\langle\varepsilon_{\mathrm{k}}^{2}\right\rangle\left|\hat{\mathrm{m}}_{\mathrm{k}}(\mathrm{q})\right|^{2} \\
& =I_{m}(q)+I_{d}(q)
\end{aligned}
$$

where $z$ denotes the partition function accounting for all the possible fluctuations, and $\hat{m}_{\mathrm{o}}(q)$ and $\hat{m}_{k}(q)$ are the complex scattering amplitudes of the meanfield structure and of the $k$-th fluctuation mode $e_{k}$, respectively. $\varepsilon_{k}$ is a real value with a zero-average Gaussian probability distribution therefore the second term in equation 4.19 vanishes.

To investigate the scattering pattern of the defect, we first study the main scattering of the defective and defect-free structures; subsequently, we analyze 
the interference of lamella fluctuations on the scattering of the defect by considering the diffusive scattering term $\mathrm{I}_{\mathrm{d}}(\mathrm{q})$.

Figure 4.23 depicts the main scattering pattern for the perfect lamella and that for the defect, $S(q)_{\text {LAM }}$ and $S(q)_{\text {DEF }}$ respectively, where periodic peaks along $\mathrm{q}_{\mathrm{y}}=0$ correspond to Bragg peaks of the lamellar structure. In the lamellar phase, changes in local composition are only normal to the AB interfaces, i.e., the $X$ direction in Figure 4.23 top-left, therefore the scattering pattern for the lamella is a rather simple one which can be easily predicted and understood.

In contrast to $S(q)_{\text {LAM }}$, the defect scattering pattern, $S(q)_{\text {LAM }}$, is an intricate continuous spectrum of wave vectors (see Figure 4.23 bottom-right). This intricate scattering pattern contains the reciprocal-space description of the topology of the defect, which is characterized by the distance between the defect cores and the angle between the line connecting both cores and the horizontal axis (see Figure 4.23 top-right). The symmetries of the configuration are another important aspect to consider in the study of the scattering pattern. As shown in Figure 4.23 top, the defect considered in our study is invariant under point inversion and the exchange of the A-B domains of the symmetric block copolymer. The exchange of $\mathrm{AB}$ domains does not affect the scattering pattern ${ }^{\text {iii }}$; therefore, those symmetries of the configuration imply that the scattering of the defect must be symmetric with respect to point inversion. This can be verified in the scattering pattern of the defect, which respect the 180 degree rotational symmetry, i.e., point inversion.

To facilitate the understanding of this complicated pattern, we compare the profile along $q_{y}=0$ for the defective and the lamellar scattering patterns in Figure 4.24. The relevant length scales of the system can be easily located in this scattering profile: the length of the periodic system $\mathrm{L}_{X}$, corresponding to $\left|\mathrm{q}_{\mathrm{L}} / \mathrm{q}_{\mathrm{o}}\right|=\mathrm{L}_{\mathrm{O}} / \mathrm{L}_{\mathrm{X}}=1 / 13 \simeq 0.0056$, the equilibrium domain spacing $\mathrm{L}_{\mathrm{O}}$, corresponding to $\left|q_{\mathrm{o}} / \mathrm{q}_{\mathrm{o}}\right|=1$, and the length of a grid cell in the collocation lattice $\mathrm{d}_{\mathrm{x}}$, corresponding to $\left|\mathrm{q}_{\mathrm{d} x} / \mathrm{q}_{\mathrm{o}}\right|=6$.

In the weak segregation limit, the lamellar composition profile can be approximated by using only the first Bragg peak whose wavevector is $\left|\mathrm{q}_{\mathrm{o}}\right|=$ $2 \pi / \mathrm{L}_{\mathrm{O}}$. In this one-mode approximation, the composition profile of the lamellar phase of a symmetric block copolymer is described by a cosine function whose Fourier transform consists of only one peak at the frequency $\left|q_{o}\right|$, i.e., the fundamental harmonic of that wave. As the degree of segregation increases, deviations from that one-mode description are represented by higher order modes with odd wave vectors, i.e., $\left|q_{x}\right|=n q_{o}$ where $n$ is an odd natural number given

\footnotetext{
iii given $S(q)=|\hat{m}(q)|^{2}$ and $\hat{m}(q)=\mathcal{F}[m(x)], S(q)$ is the same for $m(x)$ and $-\mathfrak{m}(\mathbf{x})$, i.e., the exchange of A-B domains in the lamellar phase of a symmetric block copolymer, which can be explained by the Babinet's principle $[129,130]$
} 

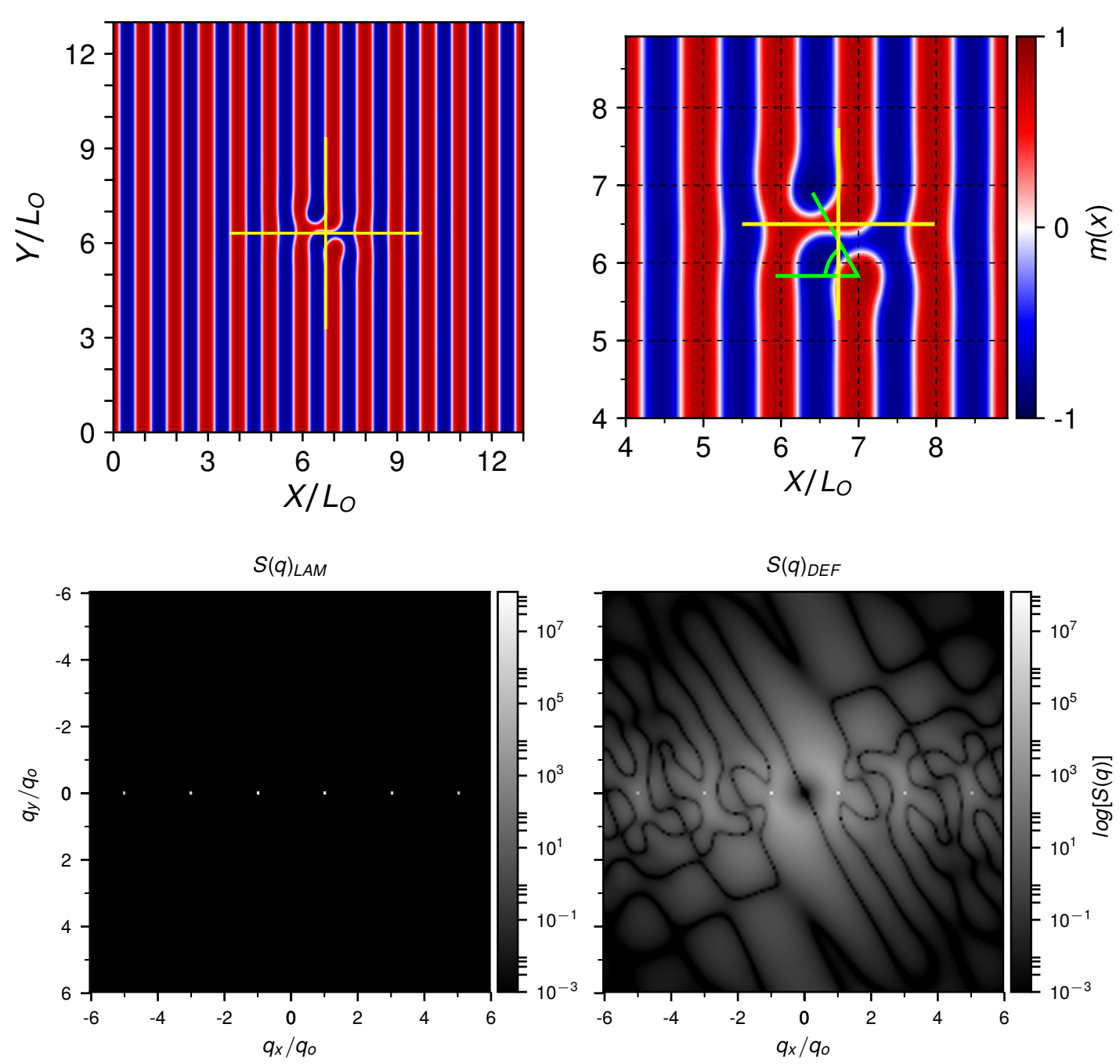

Figure 4.23: Dislocation-pair investigated in defect scattering simulation. The configuration corresponds to the local minimum of the Ohta-Kawasaki model at $\alpha=0.04638$, i.e., $\chi N \simeq 19.6$, confined in a system with periodic boundary conditions of dimensions $\mathrm{L}_{X}=\mathrm{L}_{Y}=13 \mathrm{~L}_{\mathrm{O}}$, where $\mathrm{L}_{\mathrm{O}}=13.775 \xi_{0}$ corresponds to 12 grid cells (top left). The exchange of A-B domains does not affect defect topology; thus, the defect is invariant to point inversion (yellow lines). The distance between defect cores and the angle between their connecting line and the axes are characteristic features of the defect (top right). The main scattering pattern (bottom right) is an intricate spectrum of wave vectors extending in all directions that describes the topology of the defect. This pattern includes the Bragg peaks of the lamellar structure (bottom left). Values of $S(q)<10^{-4}$ are considered negligible in all the calculations of the present section. 
(a) Scattering profile along $q_{y}=0$

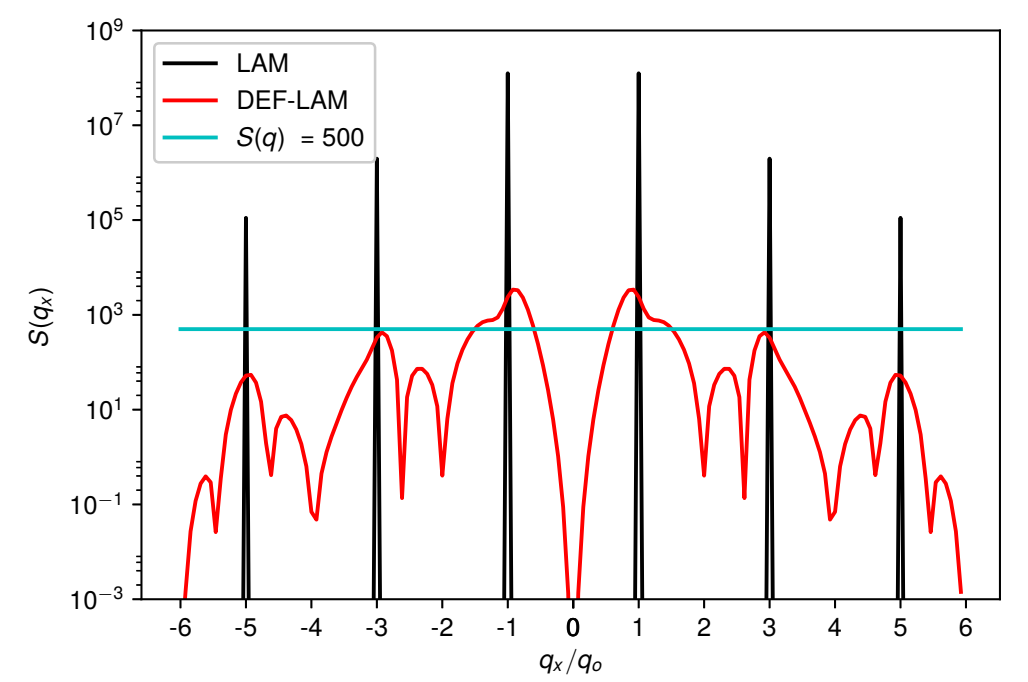

(b) Power law for $|q| \rightarrow 0$

(c) Defect scattering footprint
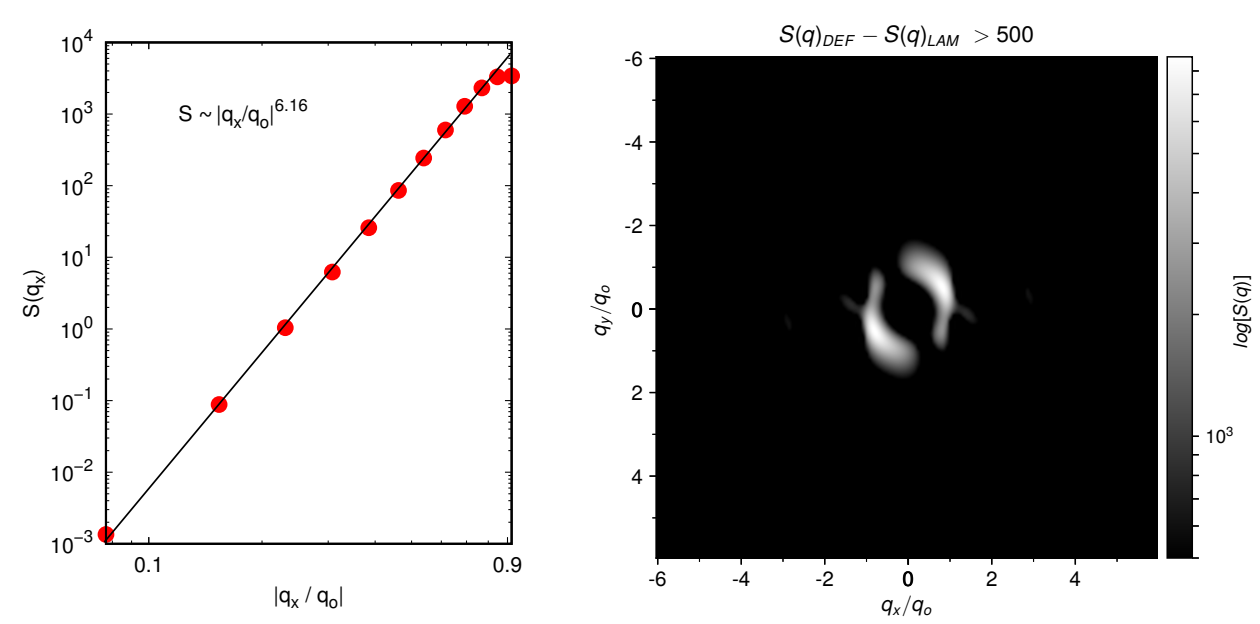

Figure 4.24: (a) A scattering profile facilitates the identification of characteristic wave vectors of the defect and the comparison with the corresponding lamellar profile. (b) Defect wave vectors with the largest $S(q)$ are located in the vicinity of the lamellar Bragg peaks. The scattering intensity at long-range wave vectors of the defect, i.e., $|q| \rightarrow 0$, obeys a power law. (c) The scattering footprint includes the largest characteristic wave vectors of the defect, i.e. $S(q)_{D E F}-S(q)_{L A M}>500$. The ratio between the scattering of the lamellar Bragg peaks is $S\left(\left|q_{o}\right|\right) / S\left(3\left|q_{o}\right|\right)=64.3$ and $S\left(\left|q_{o}\right|\right) / S\left(5\left|q_{o}\right|\right)=1163.8$ where $\mathrm{S}\left(\left|\mathrm{q}_{\mathrm{o}}\right|\right)=1.24 \times 10^{8}$. 
the symmetry of that fundamental harmonic.

The Bragg peaks at odd multiples of $\mathrm{q}_{\mathrm{o}}$ observed in Figures 4.23 and $4.24 \mathrm{a}$ describe that same symmetry in the composition profile of our configuration at an intermediate segregation regime. The ratio of the amplitude of the first to the higher order modes required for an accurate representation of the composition profile, provides a measure of the segregation degree of the block-copolymer. By considering the first two Bragg peaks, the ratio $S\left(q_{o}\right) / S\left(3 q_{o}\right)$ is maximum in the limit of the weak segregation regime.

In the strong segregation limit (SSL), the composition profile is described by a square wave whose Fourier transform contains only odd harmonics given the symmetry of the cosine wave as the fundamental harmonic required to construct such a sharp symmetric wave. In this limiting case, the decay of the corresponding Fourier amplitudes is $\sim 4 /(\pi n)$; thus, $S\left(n q_{o}\right) \sim 1 / n^{2}$ and the ratio between the Bragg peak follows $S\left(q_{o}\right) / S\left(n q_{o}\right)>n^{2}$, e.g., $S\left(\left|q_{o}\right|\right) / S\left(3\left|q_{o}\right|\right)>6$ and $S\left(\left|q_{o}\right|\right) / S\left(5\left|q_{o}\right|\right)>25$. The corresponding scattering ratios of the lamellar structure in our calculations are $S\left(\left|q_{o}\right|\right) / S\left(3\left|q_{o}\right|\right)=64.3$ and $S\left(\left|q_{o}\right|\right) / S\left(5\left|q_{o}\right|\right)=1163.8$, which satisfy the latter prediction and confirm the configuration represents a lamella in the intermediate segregation regime.

In a similar fashion, one can proceed with the study of the defect pattern using the most dominant wave vectors, i.e., $q$ with $S(|q|)>S\left(3 q_{o}\right)$. To locate those wave vectors, we discard the Bragg peaks of the lamellar phase and analyze only the scattering intensities associated with the presence of the defect in the structure, i.e, we study $S(q)_{D E F}-S(q)_{\text {LAM }}$ as shown in Figure 4.24 top. The second largest peak characteristic of the defect in the scattering profile is $S\left(q_{x}\right)=500$ and is located in the vicinity of the second Bragg peak of the lamella, i.e., $\left|q_{x}\right|<3 q_{o}$. Therefore, wave vectors with $S(q)>500$ correspond to the maximum scattering values characteristic of the defect. Figure 4.24 bottom presents the scattering pattern corresponding to those characteristic wave vectors, in other words., the defect scattering footprint.

This footprint corresponds to a simplified description of the corresponding defect. Such a description can be obtained by taking the inverse Fourier transform of the complex scattering amplitudes of the defect footprint. In this regard, Figure 4.25 shows the real-space reconstruction of the defect scattering footprint, which captures the essential topological features of the investigated defect presented in Figure 4.23 top-right).

Another important region of the scattering pattern of the defect, is composed by the wave vectors in the region $|q|<q_{0}$, which describe features of dimensions larger than the natural domain spacing of the block copolymer, $\mathrm{L}_{\mathrm{O}}$, and up to the system size $L_{x}$. The long-range strain field created by the defect in the structure is included in that long-range region of wave vectors which can 

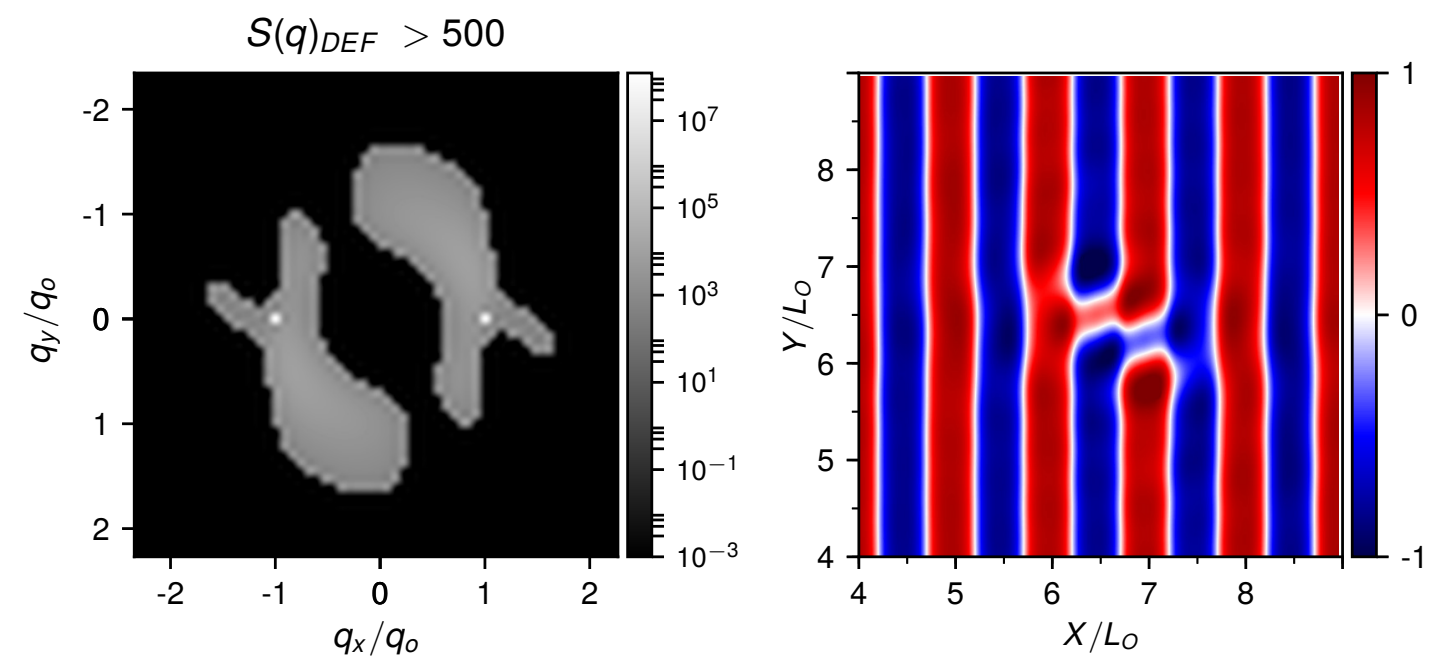

Figure 4.25: First characteristic region: Defect scattering footprint. This region comprises the wave vectors present in the scattering pattern due to the defect and which have the largest $S(q)$. Thus, the footprint (left) can be used for a simplified representation of defect topology (right) together with the first Bragg peak of the lamellar phase.

be detected using light scattering. The latter can be exploited to scan the entire silicon wafer using light scattering performing a pre-identification of regions in the lamellar structure that may contain defects. Those potentially defective regions can be further analyzed with the more precise X-ray scattering technique which has been used to characterize defects [128].

Therefore, we extract the wave vectors $|\mathrm{q}|<\mathrm{q}_{\mathrm{o}}$ from the scattering pattern of the defect (cf. Fig 4.23 bottom-right) and proceed with the corresponding real-space reconstruction. In Figure 4.26 we present the long-range scattering pattern of the defect and the corresponding real-space representation, which could be observed with longer-wavelength scattering techniques in comparison with the commonly used X-rays scattering. From the scattering pattern $|q|<q_{o}$ and the corresponding real-space reconstruction (cf. Fig 4.26a), a power law is obtained describing both decays, i.e., $S\left(q_{x}\right) \sim q_{x}^{6.16}$ and $m(X) \sim X^{-8.21}$. These exponents are characteristics of each defect topology and can be used for comparison with the displacement field of the corresponding defect providing insights for defect classification. For this latter task, previous results for liquid crystals systems can be used [111,131-133], but additional information and further analyses that fall out of the scope of the present work are required.

Hitherto, we have discussed the main scattering $I_{m}(q)$ of the defect-free and defective structures. The diffusive scattering $I_{d}(q)$ of the lamellar structure, 
(a) $|\mathrm{q}|<\mathrm{q}_{\mathrm{o}}$
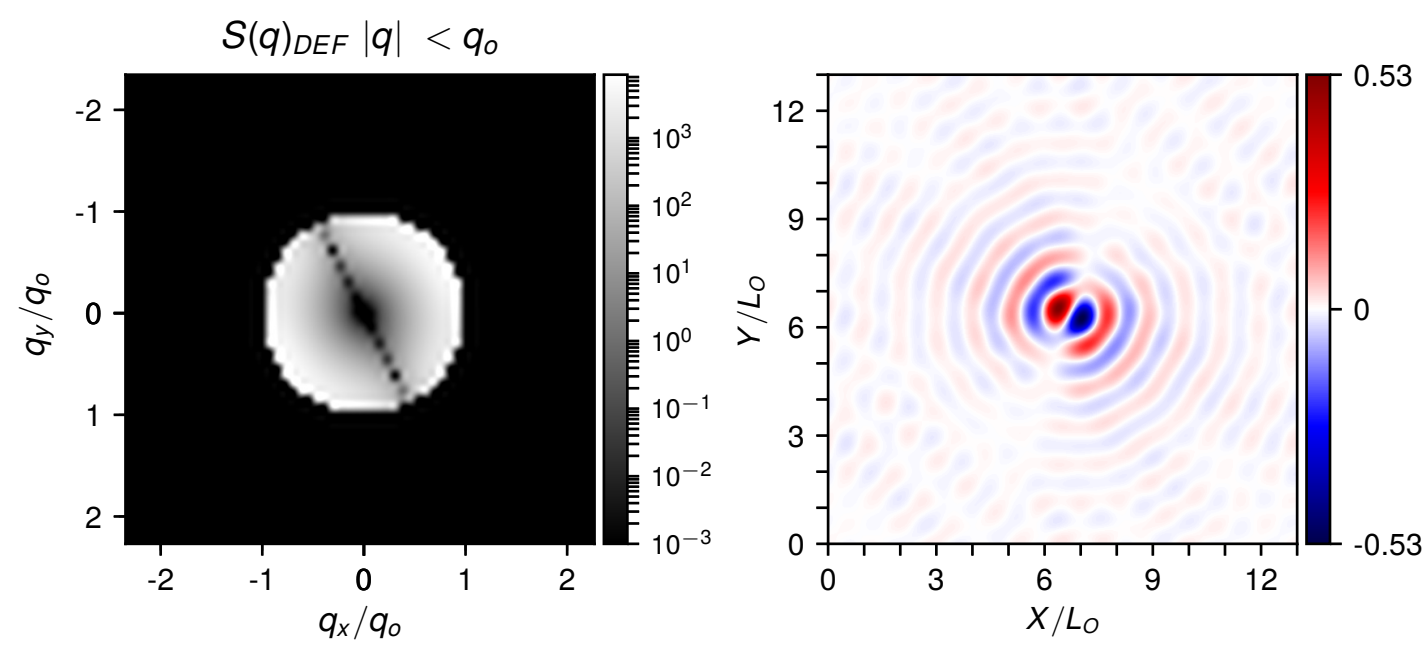

(b) $|q|<0.5 q_{o}$
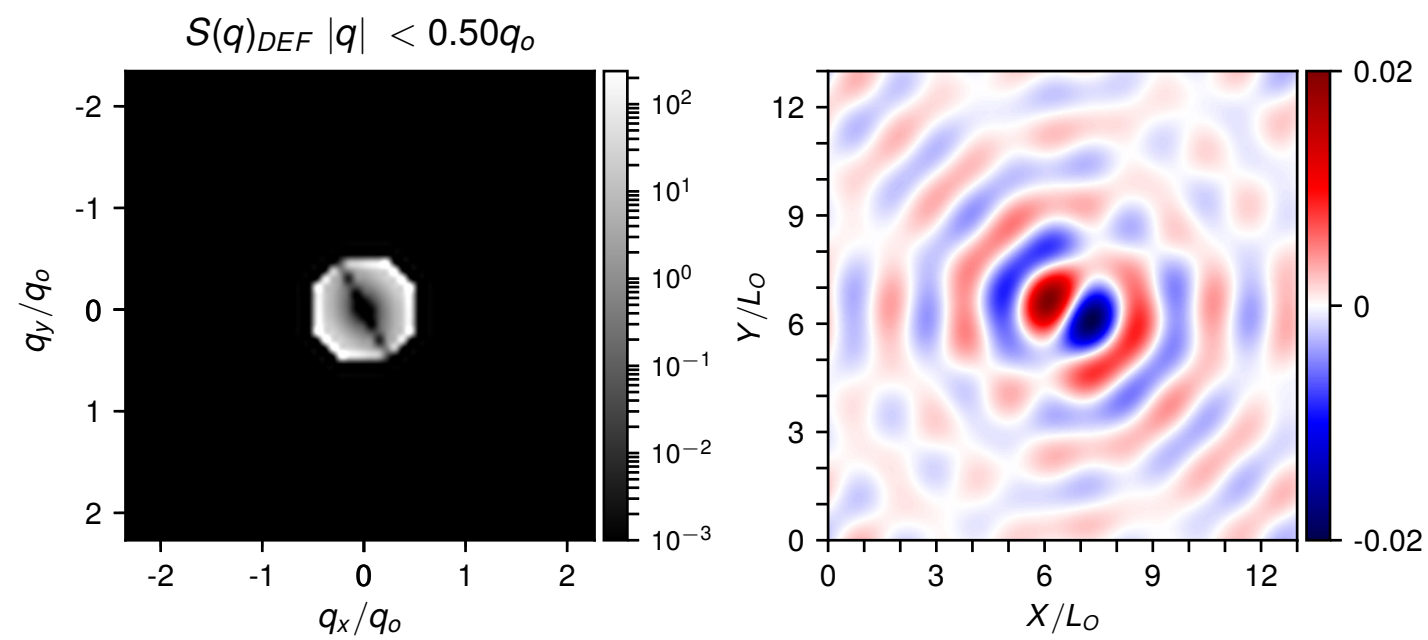

Figure 4.26: Second characteristic region: defect long-range wave vectors (left) provide a description of features of the configuration with dimensions between the natural domain spacing $\mathrm{L}_{O}$ and the system size $\mathrm{L}_{X}$. A real-space pattern (right) is obtained via the inverse Fourier transform of the complex scattering amplitude $\hat{m}(q)$ truncated to include only those long-range wave vectors. (a) For $|q|<q_{o}$, a power law describes the decay of the structure factor (left) $S\left(q_{x}\right) \sim$ $q_{x}^{6.16}$ (see Fig. 4.24b) as well as the corresponding decay in real space (right) $\mathrm{m}(\mathrm{X}) \sim \mathrm{X}^{-8.21}$ 
due to its fluctuations, can mask the scattering of the defect hindering its detection. By allowing the study of each mode independently, the fluctuation modes approach enables us to investigate which specific modes of the lamella would contribute most to such masking due to $I_{d}$.

We investigate this fluctuation-masking effect in a two-steps procedure: first, we identify the fluctuation modes of the lamella that exhibit scattering amplitudes within the two characteristic regions of the defect scattering pattern. Consequently, we analyze the influence of the magnitude of those fluctuations on the interference with the scattering of the defect, by predicting the limit of the invariant degree of polymerization, $\overline{\mathcal{N}}$, for which the masking will occur.

To scout every lamellar fluctuation mode, we discard the Bragg peaks corresponding to $\mathrm{I}_{\mathrm{m}}$ and consider $\varepsilon=1$ in order to identify wave vectors of the fluctuation mode that are located inside the defect footprint and the defect long-range pattern. By doing so, the equation 4.20 is transformed into $\mathrm{I}(\mathrm{q})=$ $\left|\hat{m}_{k}(q)\right|^{2}$, namely the structure factor of every fluctuation mode.

The lamellar fluctuation modes with wave vectors in the two aforementioned regions and with the largest values of $S(q)$ are presented in Figure 4.27: modes $k=28,80,143$ for the long-range region $\left|q_{x}\right|<q_{o}$, and modes $k=$ 134,136 around the maximum of the defect scattering footprint located at $\left|q_{x}\right|=$ $0.9 q_{o}$ and $\left|q_{y}\right|=0.5 q_{o}$, i.e., $|q|=1.03$.

All the identified lamellar fluctuation modes posses peaks located inside defect scattering footprint, i.e., $S(q)>500$. A 2D representation of selected overlapping fluctuation modes, their corresponding scattering pattern and the overlap with the defect scattering footprint is presented in Figure 4.28. A better understanding of these fluctuations is achieved by investigating the undulations and displacements that $\mathrm{AB}$ interfaces undergo due to each mode. In Figure 4.29 we present the displacement of the interfaces (red contour lines) with respect to the unperturbed lamella (black contour lines) for the four fluctuation modes identified. As can be observed by the deviation from the natural spacing $L_{O}$, the compression or stretching of some of the lamellae in the structure is induced by all of those fluctuations modes. Therefore, each one of those fluctuation modes has an associated compression wave in the $X$ direction with a different period.

Furthermore, to address the question if the lamellar fluctuation modes could hinder the detection of the defect we must consider these fluctuations in the case of an infinitely large system.

As we have previously shown (cf. Fig. 4.8), the free energy of a compression mode remains unchanged when the wave vector in the direction parallel to the $A B$ interfaces approaches zero, i.e., the system size in the $Y$ direction approaches the thermodynamic limit. We now address the question of how the compression wave of the identified fluctuation modes, cf. Fig. 4.29, changes in the limit of 


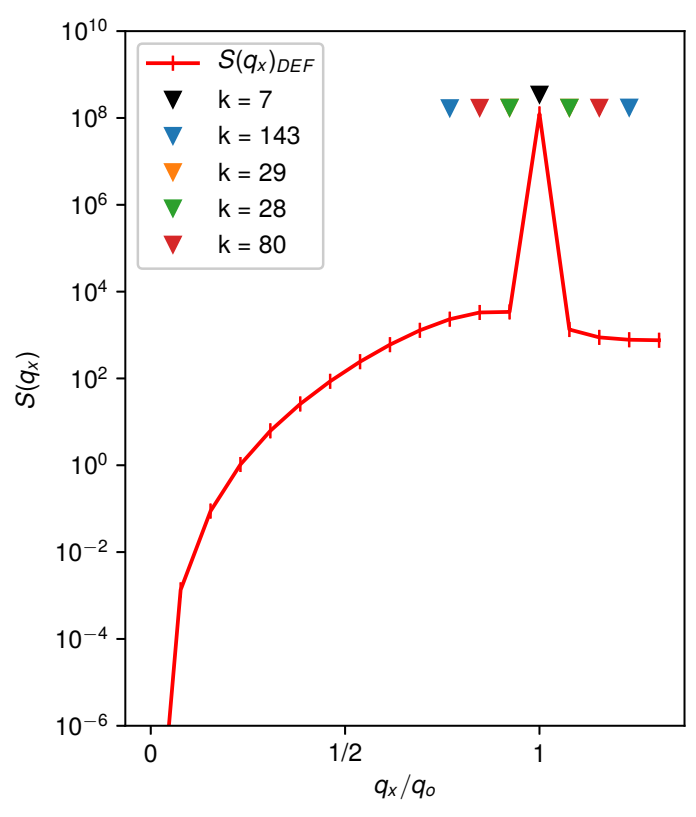

(a) $q_{y}=0$

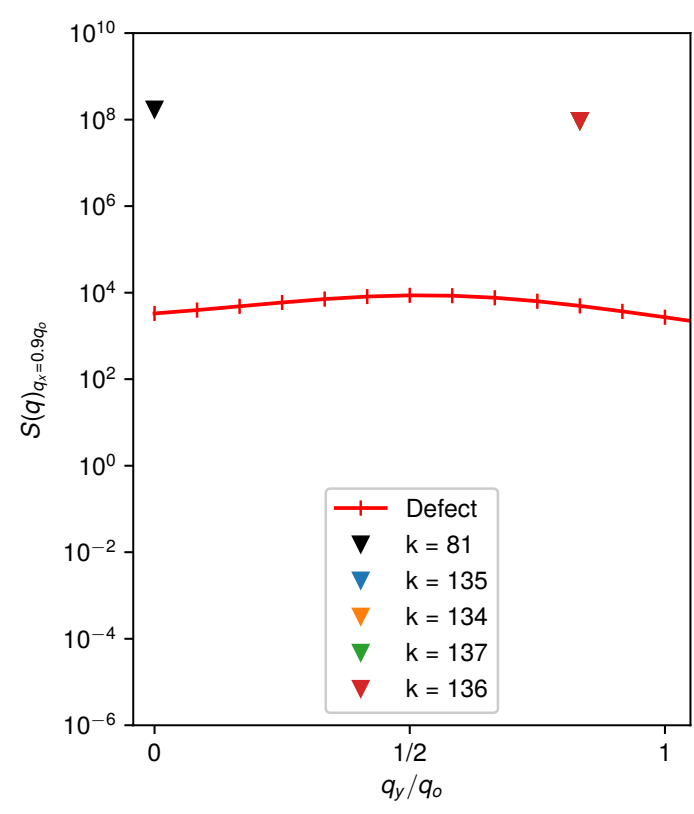

(b) $q_{x}=0.9 q_{o}$

Figure 4.27: Scattering peaks of lamellar fluctuations modes (triangles) interfere with characteristic wave vectors of the defect scattering (red line). (a) The peaks of modes $k=28,80,143$, located in the vicinity of the first Bragg peak of the lamella, were identified via scouting the long-range region of the defect scattering. (b) Peaks of modes 134 to 137 are in the vicinity of the maximum of the defect footprint located at $\left|q_{x}\right|,\left|q_{y}\right|=(0.9,0.5) q_{o}$, i.e., $|q|=1.03$. Modes $(28,29),(80,81),(134,135),(136,137)$ are degenerate pairs. 

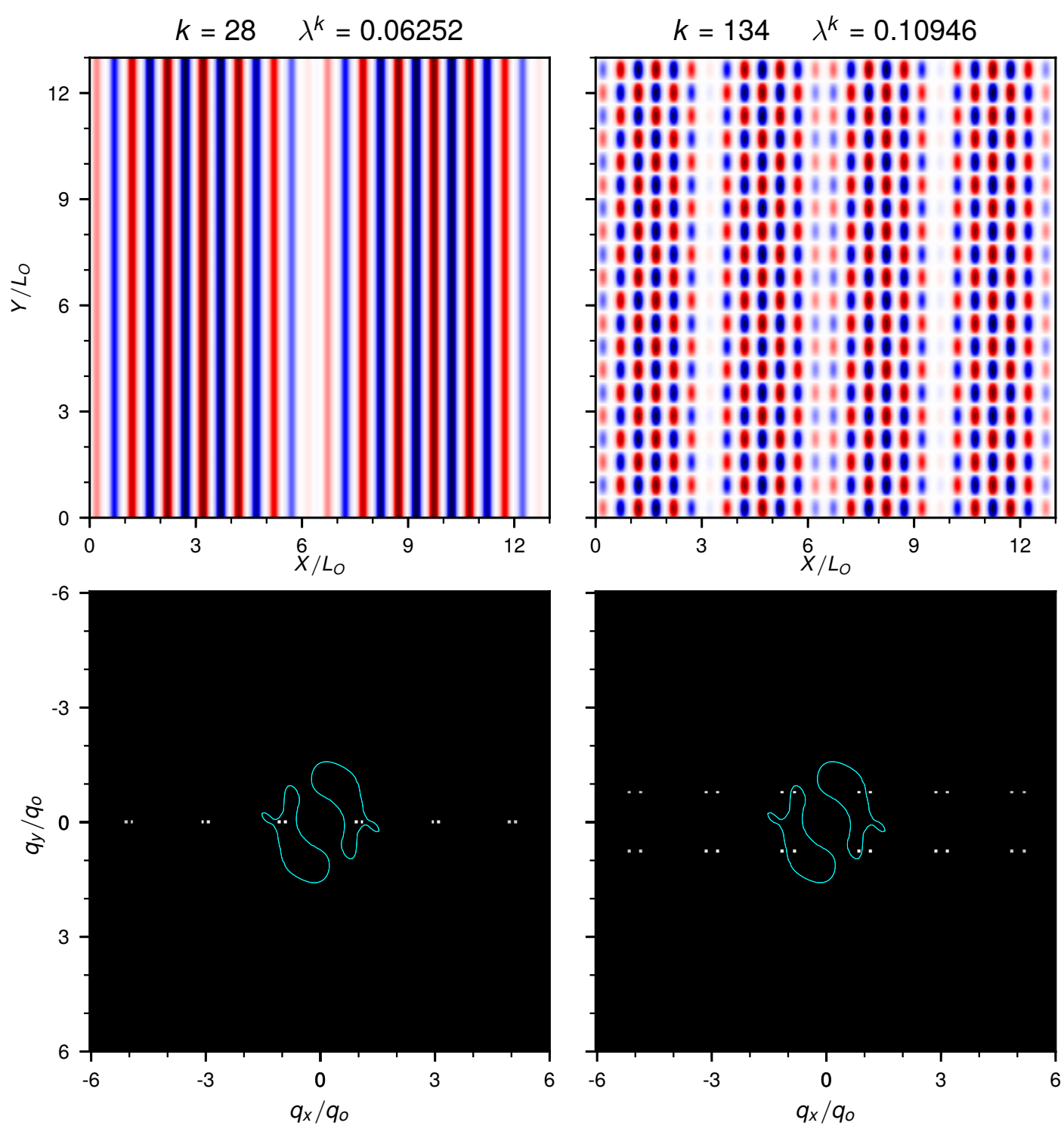

Figure 4.28: Selected fluctuation modes of the lamella that overlap the defect scattering footprint: the compression mode with the lowest eigenvalue $k=28$ and fluctuation with a scattering peak near the characteristic maximum $S(q)$ of the defect, $k=134$. White bands in the real-space representation of the modes $e_{k}(\mathbf{x})$ (top), indicate the lowest changes in local composition. Positive or negative composition changes (red and blue respectively) in the neighboring lamellae describe the stretching or compression of the lamella corresponding to the white band. The scattering pattern of each fluctuation mode $\left|\hat{m}_{k}(q)\right|^{2}$ (bottom) includes the peaks (white) that overlap the defect foot print (cyan contour). 

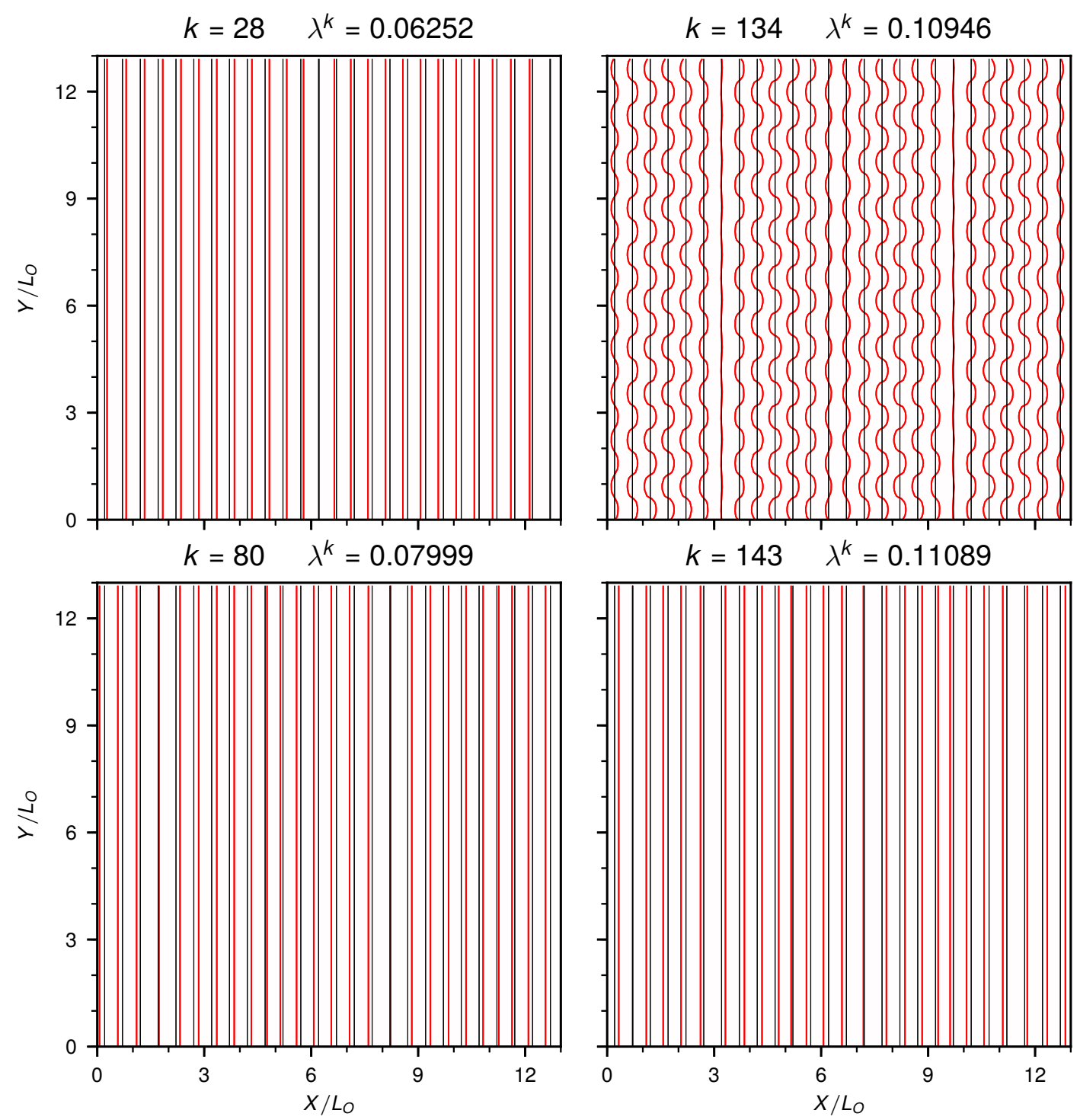

Figure 4.29: Fluctuations of the lamellar structure due to the modes that overlap the defect scattering footprint. All of the fluctuations cause compression, stretching of both in one or more lamellae. Modes $k=28,80,143$ describe the uniform displacement of the $A B$ interfaces where the difference in the direction of the displacement causes the compression and stretching, e.g., lamellae located at $X / L_{O}=6$ for mode 28 and at $X / L_{O}=8$ for modes 80 and 143. Mode 134 describes undulations of the $\mathrm{AB}$ interfaces where some of those are in phase and will vanish in the thermodynamic limit, but some others create peristaltic motion and will remain, e.g., undulations at $X / L_{O}=3,6.5$ and 10 . 
$\mathrm{q}_{x} \rightarrow 0$

The energy of those longitudinal phonons can be described by a harmonic approximation in terms of the position of the interfaces, i.e. $x_{i}$, the deviation of the lamellar spacing from the equilibrium value, i.e., $\Delta \mathrm{L}=\left(x_{i+1}-x_{i}\right)-\mathrm{L}_{\mathrm{O}}$, and the compression modulus $B$. The position of the interface $i$ is described as $x_{i}=i L_{O}+u_{i}$, where $u_{i}$ is the displacement from the equilibrium position that can be expanded as a sum of plane waves and used to calculate $\Delta \mathrm{L}$

$$
\begin{aligned}
u_{i} & =\sum_{k} a_{k} e^{2 \pi i \frac{j}{N_{x}} k} \quad \text { where } a_{k} \text { is real, thus } a_{k}=\bar{a}_{-k} \\
\Delta L & =u_{i+1}-u_{i}=\sum_{k} a_{k} e^{2 \pi i \frac{j}{N_{x}} k}\left(e^{\frac{2 \pi i}{N_{x}} k}-1\right)
\end{aligned}
$$

Hence, the free energy of the longitudinal compression or stretching is given by

$$
F=\frac{1}{2} B \sum_{i}^{N_{x}-1}(\Delta L)^{2}=\frac{1}{2} B \sum_{i}^{N_{x}-1}\left(u_{i+1}-u_{i}\right)^{2}
$$

where

$$
\begin{aligned}
\sum_{j}\left(u_{j+1}-u_{j}\right)^{2} & =\sum_{j} \sum_{k} a_{k} e^{2 \pi i \frac{j}{N_{x}} k}\left(e^{\frac{2 \pi i}{N_{x}} k}-1\right) \sum_{l} a_{l} e^{2 \pi i \frac{j}{N_{x}} l}\left(e^{\frac{2 \pi i}{N_{x}} l}-1\right) \\
& =\sum_{k l} a_{k} a_{l}\left(e^{\frac{2 \pi i}{N_{x}} k}-1\right)\left(e^{\frac{2 \pi i}{N_{x}} l}-1\right) \sum_{j} e^{\frac{2 \pi i}{N_{x}}(k+l) j}
\end{aligned}
$$


for the case $k=-l$, the last sum results in $N_{x} \delta_{k=-l}$, thus

$$
\begin{aligned}
& =N_{x} \sum_{k} a_{k} a_{-k}\left|e^{\frac{2 \pi i}{N_{x}} k}-1\right|^{2} \\
& =N_{x} \sum_{k}\left|a_{k}\right|^{2}\left(\left(\cos \left(\frac{2 \pi}{N_{x}} k\right)-1\right)^{2}+\sin ^{2}\left(\frac{2 \pi}{N_{x}} k\right)\right) \\
& =N_{x} \sum_{k}\left|a_{k}\right|^{2} 2\left(1-\cos \left(\frac{2 \pi}{N_{x}} k\right)\right) \\
& =N_{x} \sum_{k}\left|a_{k}\right|^{2} 2\left(1-1+\frac{1}{2 !}\left(\frac{2 \pi}{N_{x}} k\right)^{2}-\frac{1}{4 !}\left(\frac{2 \pi}{N_{x}} k\right)^{4}+\ldots\right) \\
& \simeq N_{x} \sum_{k}\left|a_{k}\right|^{2}\left(\frac{2 \pi}{N_{x}} k\right)^{2} \\
\sum_{j}\left(u_{j+1}-u_{j}\right)^{2} & \simeq \frac{4 \pi^{2}}{N_{x}} \sum_{k}\left|a_{k}\right|^{2} k^{2}
\end{aligned}
$$

Therefore, the free energy is given by

$$
\mathrm{F} \simeq \frac{2 \pi^{2}}{\mathrm{~N}_{\mathrm{x}}} \sum_{\mathrm{k}}\left|\mathrm{a}_{\mathrm{k}}\right|^{2} \mathrm{k}^{2}
$$

The latter is proportional to the longitudinal wavevector, i.e., $F \sim q_{x}^{2}$ for the system described herein which implies that in the limit of $k \rightarrow 0$,i.e., for the case or large systems, the energy cost of the compression modes of the lamella decays and those fluctuations will be present in a real size lamellar structure.

Therefore, the identified fluctuation modes of the lamella are to be detected via scattering and its final role in masking the scattering of the defect will be dictated by the magnitude of thermal fluctuations, i.e., the influence of $\left\langle\varepsilon^{2}\right\rangle$ in equation 4.20 on the balance between the scattering intensities of the fluctuating lamella and the intensity of the defect scattering footprint.

In this regard, the scattering of the fluctuating lamella due to a given fluctuation mode $k$, i.e., $\left.I_{(} q\right)_{L+k}$, must be smaller than the scattering intensity of the defect to facilitate the detection. Thus, considering only the main scattering of the defect, we investigate the limit $I_{m}(q)_{D}>I(q)_{L+k}$.

$$
\begin{aligned}
I_{m}(q)_{D} & >I_{m}(q)_{L}+I_{d}(q)_{L} \\
& >I_{d}(q)_{L} \\
\left|\hat{m}_{o}(q)_{D}\right|^{2} & >\left\langle\varepsilon_{k}^{2}\right\rangle\left|\hat{m}_{k}(q)\right|^{2}
\end{aligned}
$$


where $I_{m}(q)_{L}$ corresponds to the Bragg peaks of the lamellar structure which do not contribute to the overlap with the scattering pattern of the defect, therefore is discarded, and $\hat{m}_{o}(q)_{D}, \hat{m}_{k}(q)$ correspond to the complex scattering amplitude of the mean-field description of the defect and of the lamellar fluctuation mode $k$, respectively.

Considering the free energy of the fluctuating lamella $\mathcal{F}\left[\mathrm{m}_{\mathrm{k}}\right]=\mathcal{F}\left[\mathrm{m}_{\mathrm{o}}\right]+$ $\varepsilon^{2} \lambda / 2$ and resorting to the mapping between the free energy computed in the continuum model and the one obtained via SCFT, $\left\langle\varepsilon_{k}^{2}\right\rangle$ can be expressed in terms of the invariant degree of polymerization $\overline{\mathcal{N}}$, which controls fluctuation effects in the coarse-grained description of the block copolymer system.

$$
\begin{aligned}
\left\langle\varepsilon_{k}^{2}\right\rangle & =\frac{1}{z} \int \mathrm{d} \varepsilon \varepsilon_{k}^{2} e^{-\mathcal{F}\left[m_{k}\right] / k_{B} T} \quad \text { with } \quad z=\int \mathrm{d} \varepsilon e^{-\mathcal{F}\left[m_{k}\right] / k_{B} T} \\
& =\frac{k_{B} T}{\hat{\lambda}_{k}}
\end{aligned}
$$

where $\hat{\lambda}_{k}$, the eigenvalue in units of $k_{B} T$, can be obtained considering a thin film of thickness $D$ and the eigenvalue computed with the continuum model $\lambda_{k}$ as follows

$$
\frac{\hat{\lambda}_{\mathrm{k}}}{\mathrm{k}_{\mathrm{B}} \mathrm{T}}=\left(\frac{\mathrm{D}}{\mathrm{R}_{\mathrm{e}}} \frac{\eta_{0}}{\sqrt{\mathcal{N}}}\right) \sqrt{\overline{\mathcal{N}}} \lambda_{\mathrm{k}} \text { with } \quad \eta_{0}=\frac{\mathcal{F}_{\mathrm{SCFT}}[\Psi]}{\mathcal{F}_{\mathrm{OK}}[\mathrm{m}]}
$$

that yields to the expression

$$
\left\langle\varepsilon_{\mathrm{k}}^{2}\right\rangle=\frac{1}{\sqrt{\overline{\mathcal{N}}} \lambda_{k}} \frac{\sqrt{\overline{\mathcal{N}}}}{\eta_{0}} \frac{R_{\mathrm{e}}}{\mathrm{D}}
$$

Fluctuations of the lamellar phase would hinder, i.e., $\left\langle\varepsilon_{k}^{2}\right\rangle<1$, or facilitate, i.e., $\left\langle\varepsilon_{\mathrm{k}}^{2}\right\rangle>1$, the detection of the defect via scattering according to equation 4.21 and the later expression enables us to calculate the limiting value of $\overline{\mathcal{N}}^{*}$, for which $\left\langle\varepsilon_{\mathrm{k}}^{2}\right\rangle=1$. Thus, large values of $\overline{\mathcal{N}}$ will damp fluctuations and facilitate the use of scattering in defect detection, i.e., the condition $\overline{\mathcal{N}}>\overline{\mathcal{N}}^{*}$ must be satisfied.

The free energy scale, $\eta_{0}$, can be expressed in terms of the parameters of the continuum model, where for the case of the symmetric copolymer $\tilde{\alpha}=$ $48 /(2 \times N-7.1)^{2}$ and $\tilde{\gamma}=0$, yielding to

$$
\frac{\eta_{0}}{\sqrt{\overline{\mathcal{N}}}}=\frac{\sqrt{2 \chi \mathrm{N}-7.1}}{135.58}=\frac{1}{135.58}\left(\frac{48}{\tilde{\alpha}}\right)^{\frac{1}{4}}
$$


and

$$
\overline{\mathcal{N}}^{*}=\frac{\tilde{\alpha}^{\frac{1}{2}}}{\lambda_{\mathrm{k}}^{2}}\left(\frac{\mathrm{R}_{\mathrm{e}}}{\mathrm{D}}\right)^{2} 2653.28
$$

Considering the case of a small film thickness, e.g., $\mathrm{D} / \mathrm{R}_{\mathrm{e}}=1$, the limiting value for the lowest fluctuation mode of the lamella previously identified $(k=$ 28 in Fig. 4.29) is $\overline{\mathcal{N}}^{*}=1.462 \times 10^{5}$. Typical values of the invariant degree of polymerization, of the order of $\sim 10^{4}$ [5], are below that limit. A small $\overline{\mathcal{N}}$, i.e. $\overline{\mathcal{N}}<\overline{\mathcal{N}}^{*}$, will enhance fluctuations and thus the scattering of the compression modes of the lamella will mask the defect scattering.

For a thicker film of $\mathrm{D} / \mathrm{R}_{\mathrm{e}}=10, \overline{\mathcal{N}}^{*}$ drops two orders of magnitude leading to $\overline{\mathcal{N}}>\overline{\mathcal{N}}^{*}$ satisfying the aforementioned condition. Hence, an increase of the film thickness reduces $\overline{\mathcal{N}}^{*}$ and thus fluctuations will be damped facilitating the detection of the defect via scattering. The nowadays demand of block copolymers with smaller values of their natural domain spacing draws special attention to this case where films with $D / R_{e}>>1$ become experimentally relevant. In this latter case, the condition $\overline{\mathcal{N}}>\overline{\mathcal{N}}^{*}$ is easier to satisfy.

The previous prediction for the limit of $\overline{\mathcal{N}}$ considers only the main scattering of the defect, $I_{m}(q)$. Considering the additional positive diffusive term $I_{d}(q)$, the scattering intensity of the defect footprint is augmented by its thermal fluctuations as described by the scattering of the corresponding fluctuation modes, Figure 4.30. Thus, defect fluctuations enhance its scattering pattern and facilitate defect detection.

The herein prediction provides valuable insights to study defect detection using scattering, where we have identified the type of fluctuations that contribute most to the scattering of the lamellar phase in the range of length scales longer than $\mathrm{L}_{\mathrm{O}}$ that could be detected using light scattering, i.e., $|\mathrm{q}|<\mathrm{q}_{\mathrm{o}}$, like the strain field created by the defect on the lamellar structure. In addition, we have identified the limit of $\overline{\mathcal{N}}$ where the scattering of the defect is stronger than that of the thermal fluctuations of the defect-free structure, therefore facilitating defect detection via light scattering.

In this section we have presented how a continuum description of the block copolymer system, in particular via the Ohta-Kawasaki model, can be used to investigate the diffraction pattern of topological defects that can be present in a DSA experimental setting. We have deployed a dislocation pair defect to describe the particular aspects of the scattering pattern and to investigate the characteristic wave vectors of the defective structure. This analysis can be extended to other prototypical defects in order to construct a library of long-range patterns, that can be observed using optical techniques, and of defect footprints as the input for developing a procedure for the pre-identification of defective re- 
(a) $q_{y}=0$

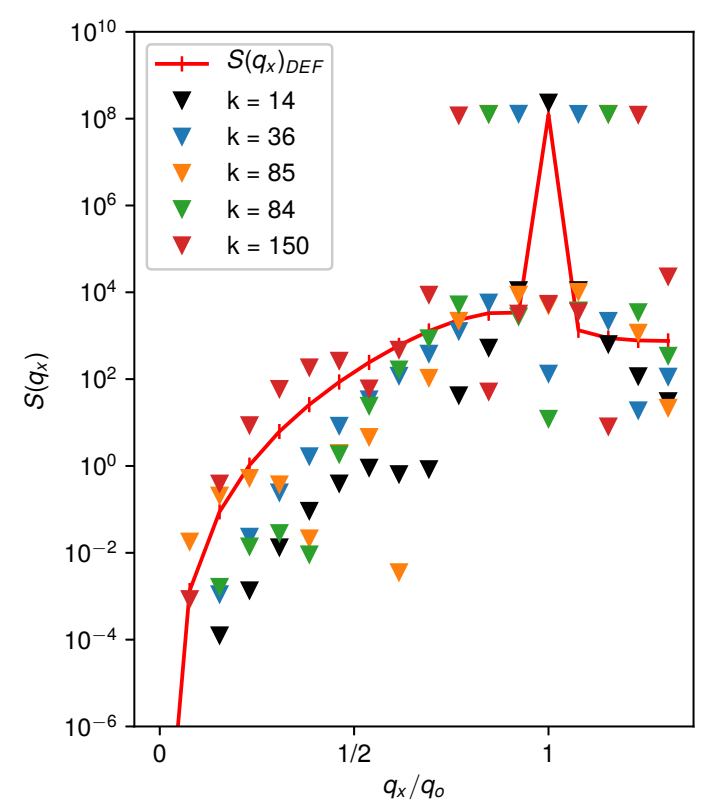

(b) $q_{x}=q_{\max }$

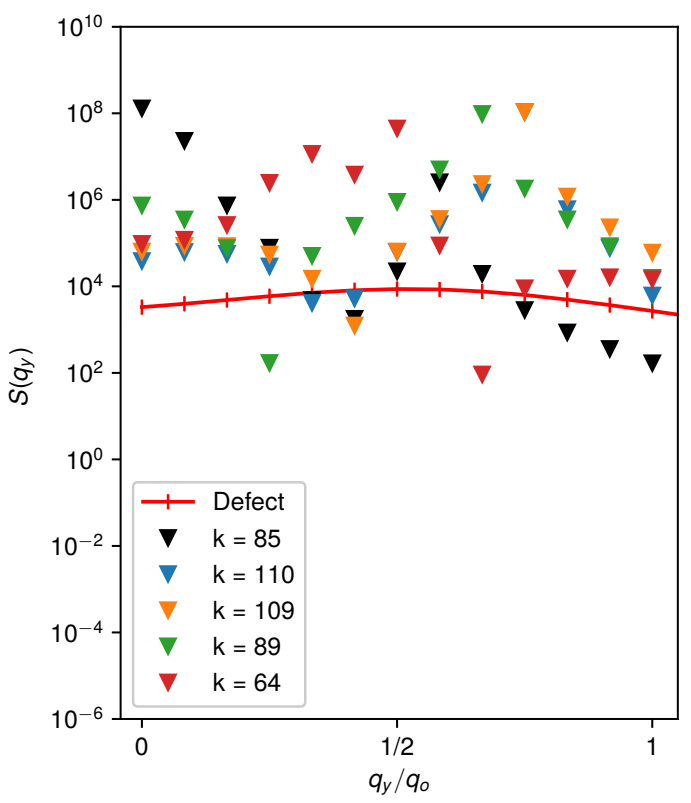

Figure 4.30: Defect fluctuations modes (triangles) amplify the main scattering the defect $S_{m}(q)$ (red line). Several modes scatter in the region of long-range wave vectors, i.e, $|q|<q_{o}(a)$, as well as around the maximum value of the scattering footprint (b). Some modes scatter in wave vectors comparable to the system size $L_{X}$, e.g, $k=150$. Fluctuations shown correspond to modes with largest scattering intensities in the two regions of interest.

gions in silicon wafers using light scattering. The comparison and investigation of the long-range patterns of various defects could allow their classification, as part of the preliminary screening of the entire wafer using light scattering. This pre-classification step could optimize the efforts in defect characterization using most expensive and time consuming techniques like SEM, AFM or X-ray scattering. However, further investigation of typical defects found in DSA, their symmetries and its effect on the scattering pattern, the scattering of structures with multiple neighboring defects as well as other aspects must be performed to develop the pre-identification and pre-characterization procedure, which is out of the scope of the present work. 


\section{Chapter 5}

\section{Final remarks and Outlook}

The development of nanostructured materials relies on the advancement of our understanding of their properties and behavior at the molecular level. To leverage the potential of block-copolymers $(\mathrm{BCP})$ as a cutting-edge technological option for industrial applications, a deeper understanding of defect formation and removal in DSA is required. To this end, reliable and fast computationally efficient modeling techniques that capture the physics of the BCP in DSA are needed to provide experts with the ability to test design ideas and explore new processing conditions.

Continuum models are suitable to fulfill that last need, and in this work, we have investigated to what extent these models can be used to describe the ordering process of diblock copolymers in the particular context of DSA. Among these models, the Ohta-Kawasaki free energy functional provides a better description of the phase diagram of diblock copolymers and the morphology and meta-stability of defects, compared to the Swift-Hohenberg model. Besides, the predictions of the Ohta-Kawasaky model for the kinetics of DSA are in good agreement with SCFT calculations and particle-based simulations. Besides the advantages mentioned above, the use of local collective densities in a continuum model drastically reduces the degrees of freedom required to describe the BCP system, and thus, the Ohta-Kawasaki model is computationally more efficient than particle-based simulations. Continuum models are also computationally more efficient than SCFT calculations due to the relatively straightforward solution of the partial differential equation that stems from the use of the free-energy functional together with the Cahn-Hilliard equation.

Due to common simplifications deployed during the implementation of the Ohta-Kasawaky model and the assumptions behind its development, some limitations can be highlighted: (i) the use of a composition-independent Onsager coefficient, $\Lambda$, hinders the accurate description of the events where the kinetic is determined by single-chain dynamics and therefore speeds up intrinsically 
slow diffusive processes. (ii) The absence of thermal fluctuations is advantageous given that solving the Cahn-Hilliard-Cook stochastic partial differential equation is a formidable task. However, no free-energy barriers can be overcome in this model due to this fact. (iii) Gaussian chain conformations are assumed by the model and encoded in the long-range kernel $G$. The presence of confinements in DSA alters the chain confirmations at these walls and how the model can account for these chain alterations has not been yet investigated.

One particularly interesting finding in our deterministic study of DSA kinetics is the influence of two periodicities in the control of the defectivity: the wavelength of the fastest growing mode $\lambda_{\max }$ and the equilibrium domain spacing $\mathrm{L}_{\mathrm{O}}$. The structure formation process can be divided into three stages: the surface-directed spinodal decomposition that prompts the formation of an initial ordered structure templated by $\lambda_{\max }$, the intermediate domain-merging stage where the periodicity of the domains changes to approach the natural value of $\mathrm{L}_{\mathrm{O}}$, and the final equilibration stage where the detailed position of the internal $\mathrm{AB}$ interfaces is optimized. As the mismatch ratio differs from unity, $\lambda_{\max } / L_{O}<1$, the intermediate stage spans for a longer period increasing the probability of the formation of undesired domains. Faster structure formation and a system less prone to defects can be promoted by tuning the DSA characteristic dimensions to $\lambda_{\max }$, such as the spacing of the guiding pattern of the size of confinement. This is achieved at low values of $\chi \mathrm{N}$ when the mismatch ratio is close to unity and the design based on the natural spacing $L_{O}$ is closer to the use of $\lambda_{\max }$ as the design length scale.

Also, we have presented the fluctuation mode analysis (FMA) as an alternative, and computationally less costly, approach to investigate local composition fluctuations within the continuum model. Beyond avoiding the efforts of solving the Cahn-Hilliard-Cook equation, the FMA allows decoupling the composition fluctuations that a given meta-stable morphology can exhibit. This feature has been exploited for the prediction of scattering patterns where specific fluctuation modes, relevant for the detection of defects via long-wavelength scattering, were identified and investigated. This finding provides the physical foundations for the design of an improved defect-metrology procedure including a preliminary screening of an entire wafer using light scattering and the preclassification of defects based on their long-range scattering footprints.

An obvious continuation of this work is to study other defect topologies commonly found in DSA to build a library of defect-scattering footprints required for the development of the aforementioned improved metrology procedure. The fabrication of line-stripe patterns via chemically-guided DSA is leading the research efforts in the next three years ${ }^{i}$, therefore defect topologies

${ }^{\mathrm{i}}$ According to results from the DSA Survey at the 4th International Symposium on DSA, 
in this systems might be a reasonable target for the continuation of this work.

Extending our findings to 3D calculations would allow the investigation of the effect of the guiding patterns on the defect removal and formation mechanism. Previous SCFT calculations have addressed this questions, and further investigations can be deployed for larger systems and unexplored topological defects to unveil their transition pathways using the FMA and the string method. Beyond the industrial interest, the computational advantages of the Ohta-Kawasaki model can be exploited to perform similar studies on the topological defects of other phases like hexagonal cylinders or gyroid aiming to draw more general conclusions for the kinetics of defect formation and annihilation.

Our work has been devoted to the study of symmetric $\mathrm{BCP}$, however, the use of asymmetric diblock-copolymers is not unusual in experimental conditions and the use of the Ohta-Kawasaki model can be extended to those systems via the extension of the phenomenological mapping that relates both model parameters, $\tilde{\alpha}$ and $\tilde{\gamma}$, to the coarse-grained invariants $f$ and $\chi N$. The extension of the Ohta-Kawasaki model to more complex molecular architectures, e.g., triblock copolymers of blends of copolymers and homopolymers is another challenge.

An important open question related to the long-range kernel, G, of the OhtaKawasaki model is how to consider boundary conditions for more complex geometries than planar surfaces. Strategies to overcome this limitation are needed to investigate topological confinements in DSA where intricate shapes can be required for the fabrication of device-oriented structures.

November 11-13, 2018, Sapporo, Japan. 


\section{Bibliography}

[1] Z. Ye, L. Xu, Z. Dong and P. Xiang, Chem. Commun., 2013, 49, 6235-6255.

[2] A. C. Rinkenauer, S. Schubert, A. Traeger and U. S. Schubert, J. Mater. Chem. B, 2015, 3, 7477-7493.

[3] M. D. Ward and M. J. Horner, CrystEngComm, 2004, 6, 401-407.

[4] D.-W. Sun and M. Müller, Macromolecules, 2018, 51, 275-281.

[5] W. H. Li and M. Müller, Annu. Rev. Chem. Biomol. Eng., 2015, 6, 187-216.

[6] J. Ren, C. Zhou, X. Chen, M. Dolejsi, G. S. W. Craig, P. A. Rincon Delgadillo, T. Segal-Peretz and P. F. Nealey, ACS Applied Materials E Interfaces, 2018, 10, 23414-23423.

[7] J. N. Murphy, K. D. Harris and J. M. Buriak, PLOS ONE, 2015, 10, 1-32.

[8] S. B. Darling, Progress in Polymer Science, 2007, 32, 1152-1204.

[9] S. O. Kim, H. H. Solak, M. P. Stoykovich, N. J. Ferrier, J. J. de Pablo and P. F. Nealey, Nature, 2003, 424, 411-414.

[10] J. Y. Cheng, A. M. Mayes and C. A. Ross, Nature Materials, 2004, 3, 823828.

[11] R. A. Segalman, Materials Science and Engineering: R: Reports, 2005, 48, 191-226.

[12] C. J. Hawker and T. P. Russell, MRS Bulletin, 2005, 30, 952-966.

[13] M. P. Stoykovich and P. F. Nealey, Materials Today, 2006, 9, 20-29.

[14] M. P. Stoykovich, H. Kang, K. C. Daoulas, G. Liu, C. C. Liu, J. J. de Pablo, M. Müller and P. F. Nealey, ACS Nano, 2007, 1, 168-175.

[15] C. T. Black, R. Ruiz, G. Breyta, J. Y. Cheng, M. E. Colburn, K. W. Guarini, H.-C. Kim and Y. Zhang, IBM J. Res. E Dev., 2007, 51, 605-633.

[16] R. Ruiz, H. M. Kang, F. A. Detcheverry, E. Dobisz, D. S. Kercher, T. R. Albrecht, J. J. de Pablo and P. F. Nealey, Science, 2008, 321, 936-939.

[17] I. Bita, J. K. W. Yang, Y. S. Jung, C. A. Ross, E. L. Thomas and K. K. Berggren, Science, 2008, 321, 939-943. 
[18] C. B. Tang, E. M. Lennon, G. H. Fredrickson, E. J. Kramer and C. J. Hawker, Science, 2008, 322, 429-432.

[19] M. Luo and T. H. Epps, Macromolecules, 2013, 46, 7567-7579.

[20] C. M. Bates, M. J. Maher, D. W. Janes, C. J. Ellison and C. G. Willson, Macromolecules, 2014, 47, 2-12.

[21] H. Hu, M. Gopinadhan and C. O. Osuji, Soft Matter, 2014, 10, 3867-3889.

[22] M. M. A., Microlelctronic Engineering, 2015, 132, 207-217.

[23] W. H. Li and M. Müller, Prog. Polym. Sci., 2016, 54-55, 47-75.

[24] L. Leibler, Macromolecules, 1980, 13, 1602-1617.

[25] A. N. Semenov, Zhurnal Eksperimentalnoi I Teoreticheskoi Fiziki, 1985, 88, 1242-1256.

[26] P. D. Olmsted and S. T. Milner, Phys. Rev. Lett., 1994, 72, 936-939.

[27] M. W. Matsen and F. S. Bates, Macromolecules, 1996, 29, 1091-1098.

[28] M. Müller and J. C. Orozco Rey, Mol. Syst. Des. Eng., 2018, 3, 295-313.

[29] M. W. Matsen, J. Phys.: Condens. Matter, 2002, 14, R21-R47.

[30] K. C. Daoulas and M. Müller, J. Chem. Phys., 2006, 125, 184904.

[31] M. Müller, K. C. Daoulas and Y. Norizoe, Phys. Chem. Chem. Phys., 2009, 11, 2087.

[32] M. Müller, J. Stat. Phys., 2011, 145, 967-1016.

[33] K. C. Daoulas, M. Müller, M. P. Stoykovich, H. Kang, J. J. de Pablo and P. F. Nealey, Langmuir, 2008, 24, 1284-1295.

[34] M. P. Stoykovich, K. C. Daoulas, M. Müller, H. M. Kang, J. J. de Pablo and P. F. Nealey, Macromolecules, 2010, 43, 2334-2342.

[35] P. N. Patrone and G. M. Gallatin, Macromolecules, 2012, 45, 9507-9516.

[36] T. Segal-Peretz, J. Ren, S. Xiong, G. Khaira, A. Bowen, L. E. Ocola, R. Divan, M. Doxastakis, N. J. Ferrier, J. de Pablo and P. F. Nealey, ACS Nano, 2017, 11, 1307-1319. 
[37] R. Ruiz, L. Wan, R. Lopez and T. R. Albrecht, Macromolecules, 2017, 50, 1037-1046.

[38] M. Müller and K. C. Daoulas, J. Chem. Phys., 2008, 128, 024903.

[39] Z. G. Wang, J. Chem. Phys., 1994, 100, 2298-2309.

[40] U. Welling, W. Li and M. Müller, SPIE Newsroom, 2014, doi $10.1117 / 2.1201403 .005354$.

[41] J. Swift and P. C. Hohenberg, Phys. Rev. A, 1977, 15, 319-328.

[42] G. H. Fredrickson and E. Helfand, J. Chem. Phys., 1987, 87, 697-705.

[43] G. H. Fredrickson and K. Binder, J. Chem. Phys., 1989, 91, 7265-7275.

[44] M. Bahiana and Y. Oono, Phys. Rev. A, 1990, 41, 6763-6771.

[45] K. R. Elder, J. Viñals and M. Grant, Phys. Rev. Lett., 1992, 68, 3024-3027.

[46] M. Seul and D. Andelman, Science, 1995, 267, 476-483.

[47] Y. Tsori and D. Andelman, Europhys. Lett, 2001, 53, 722-728.

[48] U. Thiele, A. J. Archer, M. J. Robbins, H. Gomez and E. Knobloch, Physical Review E, 2013, 87, 042915.

[49] T. Ohta and K. Kawasaki, Macromolecules, 1986, 19, 2621-2632.

[50] F. Liu and N. Goldenfeld, Phys. Rev. A, 1989, 39, 4805-4810.

[51] T. Ohta, Y. Enomoto, J. L. Harden and M. Doi, Macromolecules, 1993, 26, 4928-4934.

[52] H. Kodama and M. Doi, Macromolecules, 1996, 29, 2652-2658.

[53] S. R. Ren and I. W. Hamley, Macromolecules, 2001, 34, 116-126.

[54] C. B. Muratov, Phys. Rev. E, 2002, 66, 066108.

[55] R. Choksi and X. Ren, Journal of Statistical Physics, 2003, 113, 151-176.

[56] V. Weith, A. Krekhov and W. Zimmermann, J. Chem. Phys., 2013, 139, 054908.

[57] W. H. Li, P. F. Nealey, J. J. de Pablo and M. Müller, Phys. Rev. Lett., 2014, 113, 168301. 
[58] S. M. Hur, V. Thapar, A. Ramirez-Hernandez, G. S. Khaira, T. Segal-Perez, P. A. Ricon-Delgalilio, W. Li, M. Müller, P. F. Nealey and J. J. de Pablo, PNAS, 2015, 112, 14144.

[59] W. Li and M. Müller, Macromolecules, 2016, 49, 6126-6138.

[60] J. D. Hunter, Computing In Science E Engineering, 2007, 9, 90-95.

[61] U. Ayachit, The ParaView Guide: A Parallel Visualization Application, Kitware, Inc., USA, 2015.

[62] T. Williams, C. Kelley and many others, Gnuplot 4.6: an interactive plotting program, http://gnuplot. sourceforge.net/, 2013.

[63] P. J. Turner, Grace, http://plasma-gate.weizmann.ac.il/Grace/, (accessed October 24, 2018).

[64] M. Müller and K. C. Daoulas, Phys. Rev. Lett., 2011, 107, 227801.

[65] P. Pincus, J. Chem. Phys., 1981, 75, 1996-2000.

[66] P. G. de Gennes, J. Chem. Phys., 1980, 72, 4756-4763.

[67] E. Reister, M. Müller and K. Binder, Phys. Rev. E, 2001, 64, 041804.

[68] P. C. Hohenberg and B. I. Halperin, Rev. Mod. Phys., 1977, 49, 435-479.

[69] E. Helfand, J. Chem. Phys., 1975, 62, 999-1005.

[70] J. M. H. M. Scheutjens and G. J. Fleer, J. Phys. Chem., 1979, 83, 1619-1635.

[71] K. M. Hong and J. Noolandi, Macromolecules, 1981, 14, 727-736.

[72] M. W. Matsen and M. Schick, Phys. Rev. Lett., 1994, 72, 2660-2663.

[73] A. Arora, J. Qin, D. C. Morse, K. T. Delaney, G. H. Fredrickson, F. S. Bates and K. D. Dorfman, Macromolecules, 2016, 49, 4675-4690.

[74] H. Takahashi, N. Laachi, K. T. Delaney, S.-M. Hur, C. J. Weinheimer, D. Shykind and G. H. Fredrickson, Macromolecules, 2012, 45, 6253-6265.

[75] M. Müller, W. Li, J. C. Orozco Rey and U. Welling, MRS Proceedings, 2015, 1750, mrsf14-1750-kk03-05.

[76] S. A. Memon, PhD thesis, University of Central Lancashire, 2017. 
[77] M. Patra and M. Karttunen, Numerical methods for partial differential equations, 2006, 22, 936-953.

[78] R. C. O’Reilly and J. M. Beck, Int. J. Numer. Methods Eng, 2006, 1-16.

[79] G. J. A. Sevink, Physical Review E, 2015, 91, year.

[80] K. F. Liu and C. C. Mei, J. Fluid Mechanics, 1989, 207, 505-529.

[81] S. Glatz, Master's thesis, Georg-August University School of Science (GAUSS), 2013.

[82] A. Cavallo, M. Muller, J. Wittmer, A. Johner and K. Binder, Journal of Physics-Condensed Matter, 2005, 17, S1697-S1709.

[83] E. Krause and W. Jäger, High Performance Computing in Science and Engineering ' 04: Transactions of the High Performance Computing Center, Stuttgart (HLRS) 2004, Springer Berlin Heidelberg, 2007.

[84] A. D. Pezzutti, D. A. Vega and M. A. Villar, Philosophical transactions of the royal society A-mathematical physical and engineering sciences, 2011, 369, 335-350.

[85] Q. Tong and S. J. Sibener, Macromolecules, 2013, 46, 8538-8544.

[86] M. Peach and J. S. Koehler, Phys. Rev., 1950, 80, 436-439.

[87] M. P. Stoykovich, M. Müller, S. O. Kim, H. H. Solak, E. W. Edwards, J. J. de Pablo and P. F. Nealey, Science, 2005, 308, 1442-1446.

[88] T. Fühner, P. Michalak, U. Welling, J. C. Orozco-Rey, M. Müller and A. Erdmann, Proc. SPIE, 2016, 9780, 97800M-97800M-12.

[89] I. Schmidt and K. Binder, J. Phys. (France), 1985, 46, 1631-1644.

[90] S. Puri and K. Binder, J. Stat. Phys., 1994, 77, 145-172.

[91] P. K. Jaiswal, K. Binder and S. Puri, J. Chem. Phys., 2012, 137, 064704.

[92] M. Müller, W. H. Li, J. C. Orozco Rey and U. Welling, J. Phys.: Conf. Ser., 2015, 640, 012010.

[93] J. A. de la Torre, P. E. nol and A. Donev, J. Chem.- Phys., 2015, 142, 094115.

[94] A. Silberberg, J. Coll. Interf. Sci, 1982, 90, 86-91. 
[95] D. T. Wu, G. H. Fredrickson, J. P. Carton, A. Adjdari and L. Leibler, J. Polym. Sci.: Polym. Phys., 1995, 33, 2373 - 2389.

[96] C. Seok, K. F. Freed and I. Szleifer, J. Chem. Phys., 2004, 120, 7174-7182.

[97] A. F. Hannon, Y. Ding, W. Bai, C. A. Ross and A. Alexander-Katz, Nano Letters, 2014, 14, 318-325.

[98] G. S. Khaira, J. Qin, G. P. Garner, S. Xiong, L. Wan, R. Ruiz, H. M. Jaeger, P. F. Nealey and J. J. de Pablo, ACS Macro Letters, 2014, 3, 747-752.

[99] S. P. Paradiso, K. T. Delaney and G. H. Fredrickson, ACS Macro Letters, 2016, 5, 972-976.

[100] A. W. Bosse, Physical Review E, 2012, 85, year.

[101] J. De la Torre, P. Español and A. Donev, The Journal of Chemical Physics, 2014, 142,.

[102] W. Li and M. Müller, Annual Review of Chemical and Biomolecular Engineering, 2015, 6, 187-216.

[103] J. P. Sethna, Statistical mechanics : entropy, order parameters, and complexity, Oxford University Press, 2006, p. 349.

[104] P. M. Goldbart, S. Mukhopadhyay and A. Zippelius, Physical Review B, 2004, 70, 184201.

[105] M. Müller and G. Münster, Journal of Statistical Physics, 2005, 118, 669-686.

[106] N. J. Gunther, D. J. Wallace and D. A. Nicole, Journal of Physics A: Mathematical and General, 1980, 13, 1755-1767.

[107] Z. Wang, The Journal of Chemical Physics, 1994, 100, 2298-2309.

[108] H. T. Davis, The Journal of Chemical Physics, 1977, 67, 3636-3641.

[109] M. W. Matsen, Current Opinion in Colloid \& Interface Science, 1998, 3, 40-47.

[110] M. Sferrazza, C. Xiao, R. A. L. Jones, D. G. Bucknall, J. Webster and J. Penfold, Physical Review Letters, 1997, 78, 3693-3696.

[111] P. S. Pershan, J. Appl. Phys., 1974, 45, 1590-1604.

[112] W. E, W. Ren and E. Vanden-Eijnden, J. Chem. Phys., 2007, 126, 164103. 
[113] M. Müller and D.-W. Sun, Journal of Physics: Condensed Matter, 2015, 27, 194101.

[114] Y. G. Smirnova, M. Fuhrmans, I. A. B. Vidal and M. Müller, Journal of Physics D: Applied Physics, 2015, 48, 343001.

[115] W. E, W. Ren and E. Vanden-Eijnden, Phys. Rev. B, 2002, 66, 052301.

[116] S.-M. Hur, V. Thapar, A. Ramirez-Hernandez, G. Khaira, T. Segal-Peretz, P. A. Rincon-Delgadillo, W. Li, M. Múller, P. F. Nealey and J. J. de Pablo, Proceedings of the National Academy of Sciences of the United States of America, 2015, 112, 14144-14149.

[117] M. Laradji, A. C. Shi, J. Noolandi and R. C. Desai, Macromolecules, 1997, 30, 3242-3255.

[118] M. Laradji, A. C. Shi, R. C. Desai and J. Noolandi, Phys. Rev. Lett., 1997, 78, 2577-2580.

[119] A. C. Shi, J. Noolandi and R. C. Desai, Macromolecules, 1996, 29, 6487-6504.

[120] C. Yeung, A. C. Shi, J. Noolandi and R. C. Desai, Macromolecular Theory and Simulations, 1996, 5, 291-298.

[121] A. Ranjan, J. Qin and D. C. Morse, Macromolecules, 2008, 41, 942-954.

[122] P. Eaton, P. Quaresma, C. Soares, C. Neves, M. de Almeida, E. Pereira and P. West, Ultramicroscopy, 2017, 182, $179-190$.

[123] A. McClelland and M. Mankin, Optical Measurements for Scientists and Engineers: A Practical Guide, Cambridge University Press, 2018.

[124] P. Eaton and P. West, Atomic Force Microscopy, OUP Oxford, 2010.

[125] C. Teichert, J. Mackay, D. Savage, M. Lagally, M. Brohl and P. Wagner, Applied Physics Letters, 1995, 66, 2346-2348.

[126] M. W. D. Joel S. Silfies, Stanley A. Schwartz, The Diffraction Barrier in Optical Microscopy, 2018 (accessed October 24, 2018).

[127] R. Rottenfusser, Digital Microscopy, Academic Press, 2013, vol. 114, pp. 43 -67 .

[128] G. Khaira, M. Doxastakis, A. Bowen, J. Ren, H. S. Suh, T. Segal-Peretz, X. Chen, C. Zhou, A. F. Hannon, N. J. Ferrier, V. Vishwanath, D. F. Sunday, R. Gronheid, R. J. Kline, J. J. de Pablo and P. F. Nealey, Macromolecules, 2017, 50, 7783-7793. 
[129] Y. Nakata, Y. Urade, T. Nakanishi and M. Kitano, Phys. Rev. B, 2013, 88, 205138.

[130] G. Kubické, C. Bourlier, M. Delahaye, C. Corbel, N. Pinel and P. Pouliguen, Radio Science, 2013, 48, 573-581.

[131] M. Zapotocky and P. M. Goldbart, eprint arXiv:cond-mat/9812235, 1998.

[132] A. J. Bray, S. Puri, R. E. Blundell and A. M. Somoza, Phys. Rev. E, 1993, 47, R2261-R2264.

[133] A. P. Y. Wong, P. Wiltzius, R. G. Larson and B. Yurke, Phys. Rev. E, 1993, 47, 2683-2688. 


\section{Curriculum Vitae}

\section{Personal Data}

Name

Juan Carlos Orozco Rey

Date of birth

09.01.1979

Place of birth

Nationality

Bogotá, Colombia

Address

Colombian

Robert-Koch Str. 38, 37075, Göttingen

\section{Education}

04.2014 - Present

PhD student of Prof. Dr. Marcus Müller

Institute for Theoretical Physics

Georg-August-Universität, Göttingen

Thesis: Kinetics of Directed Self-Assembly of Block Copolymers via

Continuum Models

02.2009 - 10.2013 Master of Sciences in Biomedical Engineering

Medical School

National University of Colombia, Bogotá, Colombia

Thesis: Mesoscale simulation of mucoadhesive behavior of biopolymers and a mucin-type model protein using the DPD method

Graduated with honors

02.1996 - 10.2002 Bachelor in Chemical Engineering

Department of Chemical Engineering

National University of Colombia, Bogotá, Colombia

Thesis: Synthesis of hydrogels by inverse suspension copolymerization of acrylic acid and acrylamide for fertilizer controlled-release

Best undergraduate research thesis in Che. Eng. nationwide 2003

Göttingen, 12.2018 


\section{List of Publications}

2018 M. Müller and J.C. Orozco-Rey, Continuum Models for Directed SelfAssembly, Mol. Syst. Des. Eng.

https://doi.org/10.1039/C7ME00109F

2016 T. Fühner, P. Michalak, U. Welling, J.C. Orozco-Rey, M. Müller, and A. Erdmann, An integrated source / mask / DSA optimization approach Proc. SPIE 9780, Optical Microlithography XXIX, 97800M https://doi.org/10.1117/12.2222170

2015 M. Müller, W.H. Li, J.C. Orozco-Rey, and U. Welling, Kinetics of directed self-assembly of block copolymers on chemically patterned substrates J. Phys.: Conf. Ser. 640, 012010

2015 M. Müller, W.H. Li, J.C. Orozco-Rey, and U. Welling, Defect annihilation in chemo-epitaxial directed assembly: Computer simulation and self-consistent field theory MRS Proceedings 175

https://doi.org/10.1557/opl.2015.285

ORCID: https://orcid.org/0000-0002-2399-2474 